\title{
Recent mathematical developments in the Skyrme model
}

\author{
T. Gisiger and M.B. Paranjape \\ Groupe de Physique des Particules, Université de Montréal, C.P. 6128, succ. \\ centre-ville, Montréal, Québec, Canada, H3C 3J'7
}

\begin{abstract}
In this review we present a pedagogical introduction to recent, more mathematical developments in the Skyrme model. Our aim is to render these advances accessible to mainstream nuclear and particle physicists. We start with the static sector and elaborate on geometrical aspects of the definition of the model. Then we review the instanton method which yields an analytical approximation to the minimum energy configuration in any sector of fixed baryon number, as well as an approximation to the surfaces which join together all the low energy critical points. We present some explicit results for $B=2$. We then describe the work done on the multibaryon minima using rational maps, on the topology of the configuration space and the possible implications of Morse theory. Next we turn to recent work on the dynamics of Skyrmions. We focus exclusively on the low energy interaction, specifically the gradient flow method put forward by Manton. We illustrate the method with some expository toy models. We end this review with a presentation of our own work on the semi-classical quantization of nucleon states and low energy nucleon-nucleon scattering.
\end{abstract}

\section{Introduction}

The Skyrme model[四] was first proposed by T.H.R. Skyrme' in the sixties, as a revolutionary idea for incorporating baryons in the non-linear sigma model description of the low-energy interactions of pions. This sigma model consists of a unitary matrix valued field $U(\vec{x}, t)$ of dimension $2 \times 2$ or $3 \times 3$ depending on the number of light quark flavours that are considered. The dynamics is described by the Lagrange density

$$
\mathcal{L}=-\frac{f_{\pi}^{2}}{4} \operatorname{tr}\left(U^{\dagger} \partial_{\mu} U U^{\dagger} \partial^{\mu} U\right)
$$

\footnotetext{
${ }^{1}$ For an interesting compilation of the life, work and influence in physics of T.H.R. Skyrme, see Selected Papers, with commentary, of Tony Hilton Royle Skyrme (World Scientific Series in 20th Century Physics-Vol. 3), G.E. Brown editor
} 
where $f_{\pi}^{2}$ is the pion decay constant. Skyrme noted the existence of topologically non-trivial field configurations of finite energy. These were however, unstable against collapse, which can be adduced by simple application of scaling arguments. Skyrme then added a higher derivative term to the Lagrange density rendering these configurations stable. This term is now called the Skyrme term and the Skyrme Lagrange density $\mathcal{L}_{\text {sk }}$ is given by

$$
\mathcal{L}_{\mathrm{sk}}=-\frac{f_{\pi}^{2}}{4} \operatorname{tr}\left(U^{\dagger} \partial_{\mu} U U^{\dagger} \partial^{\mu} U\right)+\frac{1}{32 e^{2}} \operatorname{tr}\left(\left[U^{\dagger} \partial_{\mu} U, U^{\dagger} \partial_{\nu} U\right]^{2}\right)
$$

where $e$ is a new, dimensionless coupling constant. Since each derivative corresponds to a momentum, this term is clearly of higher order in the lowenergy (momentum) approximation. Skyrme proposed the interpretation of these topological solitons (stable, localized, finite-energy solutions of the classical equations of motion) as the nucleons and identified the topological winding number of the soliton with the baryon number. The technology of quantum field theory in the sixties was not sufficiently advanced to treat solitons and it took almost twenty years before the ideas of Skyrme were revived by Balachandran et al [2] and Witten[3], and vindicated with surprising accord with experiment[四]. Witten[3] described another topological density which should be added in the effective action, the celebrated Wess-Zumino-NovikovWitten 3,5,6] term

$$
\Gamma_{W Z N W}=-\frac{\mathrm{i} N}{240 \pi^{2}} \int_{D_{5}} \mathrm{~d}^{5} x \epsilon^{\mu \nu \alpha \beta \gamma} \operatorname{tr}\left[U^{\dagger} \partial_{\mu} U U^{\dagger} \partial_{\nu} U U^{\dagger} \partial_{\alpha} U U^{\dagger} \partial_{\beta} U U^{\dagger} \partial_{\gamma} U\right](3)
$$

where $U \in \mathrm{SU}(3), N \in \mathbb{Z}\left(D_{5}\right.$ is in fact a 5-dimensional manifold with only its boundary giving the usual 4-dimensional space-time). Witten[3] showed its relation to the underlying microscopic theory of the strong interactions QCD, with the number of colours giving the quantized coefficient $N$ in $\Gamma_{W Z N W}$. Since these seminal papers there has been an enormous amount of work relating the Skyrme model to phenomena in nuclear and particle physics, for instance targeting the spectrum of excitations of baryons [7], the inclusion of strange degrees of freedom in the model[8], the nucleon-nucleon potential[9, 10], scattering $\pi-N$ states[11], high density baryon matter as a Skyrme crystal [12] and the nucleon-anti-nucleon annihilation [13, 14, 15, 16, 17, 18 to name a few. We will not consider these developments in detail here and refer the interested reader to the literature and to the many excellent reviews on the subject[19,20,21,22].

Concurrently, there were certain mathematical advances in the Skyrme model which were not strongly based on making any contact with phenomenology, but moreover to understand the mathematical content of the model. These concerned two main areas, that of the exact nature of the minima or critical 
points of the static energy functional and secondly a description of the scattering of the corresponding solitons. It turns out in a certain approximation, that of low energy scattering, these two aspects are not unrelated. There were also some auxiliary mathematical and physical results, concerning geometrical insights into the model. Our review will primarily focus on these relatively recent mathematical developments. We start with the static sector and introduce the Skyrme model on a general Riemannian manifold. Then we present the methods using instantons and rational maps to obtain useful Skyrme configurations, followed by a short description of Morse theory and its application in the model. Next we move to the dynamics where we treat the gradient flow method put forward for studying soliton scattering. We terminate with it applications to the baryon number 2 sector of the model. Most of the advances which we will consider were made by N.S. Manton, among others (our original contributions to this subject are secondary). Since these advances generally use the language and formalism of differential geometry, the reader should be familiar with these notions (any standard course on tensor analysis/differential geometry/general relativity should be adequate[23, 24,25,26]).

We start the study of the statics of the model in section 3 with the geometrical aspects of the model as first discussed by Manton and Ruback 27 and then by Manton 28 and Loss 29. We show in detail how the model can be understood as a theory of elasticity in curved space. This fruitful approach allows us to explain, for example, why the Skyrmion (the lowest energy solution of the model with baryon number 1) does not saturate the Bogomolnyi bound in ordinary space, but does so for a space with great curvature. This situation is connected to chiral symmetry restoration and deconfinement. In section 3.1 we first review the theory of non-linear elasticity, both in flat and curved space, as well as some tools of differential geometry. We then move on to show how those tools can allow us to connect elasticity and the Skyrme model. This shows how it is possible to formally interpret a classical field theory as an elasticity theory.

Section 3.2 describes the instanton method put forward by Atiyah and Manton 30 to approximate critical points of the Skyrme energy functional. Indeed, the Skyrme model has always been plagued (as T.H.R. Skyrme realized already in the sixties) by the absence of analytical solutions: to obtain solutions of the model, one always has to solve partial differential equations numerically, except in the simplest cases like the $B=1$ Skyrmion, where one has only to solve an ordinary differential equation numerically. The instanton method permits one to obtain analytical expressions (in the simplest cases) or at least limit the difficulties to solving ordinary differential equations. The tradeoff is that in this method (which is by nature approximate) the error introduced is hard to estimate, except by comparing the resulting approximation with the full "exact" numerical solution of the problem. The method seems nevertheless to work reasonably well, and has been helpful in investigating the bound 
states in the sector $B=2,3$ and 4 , and Skyrme crystals as well $31,32,33$.

The next subsection 3.3 presents the use of rational maps 34 in finding approximations to minima with particular symmetries for sectors comprising of a range of baryon numbers. Rational maps are mappings of the complex plane to itself comprised of ratios of polynomials. The choice of the coefficients of the polynomials can encode the mappings with complicated symmetries, especially when viewed via stereographic projection as mappings of $S^{2} \rightarrow S^{2}$. The former $S^{2}$ corresponds to the angular degrees of freedom about a Skyrme configuration while the latter corresponds to a selected $S^{2}$ in the group $\mathrm{SU}(2)$ obtained via the Hopf projection as will be explained in subsection 3.3.

We give in subsection 3.4 a short introduction to Morse theory and how it could be used to find new solutions to the equations of motion[35]. Morse theory relates the existence of critical points of a function defined on a manifold to non-trivial topological aspects of the manifold. Here the function in question is the energy functional defined on the manifold of the space of all static field configurations. Although the method has not borne fruit in the Skyrme model, it has already been useful in the analysis leading to the sphaleron of the standard electroweak model 36.

In the next section (section 4), we present some recent developments in the study of the dynamics of the model, namely Skyrmion-Skyrmion and nucleonnucleon scattering.

We start by presenting in section 4.1 Manton's method [37] for truncating the degrees of freedom of a system, thereby possibly rendering it tractable. This formalism was published by Manton after his work on BPS monopole (topological solitons of the massless SU(2) Higgs model) scattering 38. It is a much more general method in the sense that it is applicable not only to soliton problems (namely scattering thereof) but to many systems with a great number of degrees of freedom under the right circumstances. Even though the underlying ideas are intuitively simple, the formalism needs to be expressed using differential geometry. This is why in section 4.2 we introduce this method using a number of simple examples to help us emphasize the ideas behind the method and to get a feel for how it works. Results obtained using this method are then compared with "exact" numerical results. In section 4.3 we present the application of the method to the case $B=2$ in the Skyrme model.

We close this review with a discussion of our work on Skyrmion-Skyrmion 39] and nucleon-nucleon 40] scattering using Manton's method but in a semiclassical, rather than purely quantum mechanical perspective. 


\section{The Skyrme model: Lagrangian and notation}

The Skyrme model including mass is described by the Lagrange density,

$$
\begin{aligned}
\mathcal{L}_{\text {sk }}=-\frac{f_{\pi}^{2}}{4} \operatorname{tr}\left(U^{\dagger} \partial_{\mu} U U^{\dagger} \partial^{\mu} U\right) & +\frac{1}{32 e^{2}} \operatorname{tr}\left(\left[U^{\dagger} \partial_{\mu} U, U^{\dagger} \partial_{\nu} U\right]^{2}\right) \\
& -\frac{1}{2} m_{\pi}^{2} \operatorname{tr}\left(U+U^{\dagger}\right)
\end{aligned}
$$

where $U(x)$ is a unitary matrix valued field. The Lagrangian is also often written in terms of $F_{\pi}=2 f_{\pi}$ but this is only a matter of convention. The quantum fluctuations of $U(x)$ represent the low energy mesons made up of quark-anti-quark pairs. In the chiral limit, all light quarks $(u, d, s)$ are massless and degenerate and the corresponding flavour symmetry dictates that $U(x)$ is a $3 \times 3$ matrix. In this review, however, we will be more focused on the fact that $U(x)$ is a unitary matrix; the simplest example of this is a $2 \times 2$ matrix, the only case that we will consider here. Phenomenologically, this means we consider the explicit breaking of the SU(3) symmetry to be large. The Skyrme Lagrangian (4) corresponds to the first two terms of a systematic expansion in derivatives of the effective Lagrangian describing low energy interactions of pions plus the mass term. It should be derivable from QCD hence $f_{\pi}, e$ and $m_{\pi}$, the pion mass, are in principle calculable parameters. These calculations are actually unfeasable and we take $f_{\pi}, e$ and $m_{\pi}$ from phenomenological fits. We then find $f_{\pi}$ to be in the range of $130-190 \mathrm{MeV}$ and $e \simeq 5$. In this article, we will always take $m_{\pi} \rightarrow 0$ for simplicity.

The energy functional, coming from the static part of the Lagrangian, can be written in a more elegant way by adopting a convenient choice of units (so-called natural units) [27]:

$$
E_{\text {sk }}=\int \mathrm{d}^{3} \vec{x}\left[-\frac{1}{2} \operatorname{tr}\left(U^{\dagger} \partial_{\mathrm{i}} U U^{\dagger} \partial_{\mathrm{i}} U\right)-\frac{1}{16} \operatorname{tr}\left(\left[U^{\dagger} \partial_{\mathrm{i}} U, U^{\dagger} \partial_{\mathrm{j}} U\right]^{2}\right)\right],
$$

where the unit of energy is now $f_{\pi} / 4 e \simeq 6 \mathrm{MeV}$ and the unit of length $2 / e f_{\pi} \simeq$ $0.6 \mathrm{fm}$.

The baryons arise as topological solitonic solutions of the equations of motion [1]. These topological solitons correspond to non-trivial mappings of $\mathbb{R}^{3}$ plus the point at infinity into $\mathrm{SU}(2)$ :

$$
U(\vec{x}): \mathbb{R}^{3}+\infty \rightarrow \mathrm{SU}(2)=\mathrm{S}^{3} .
$$




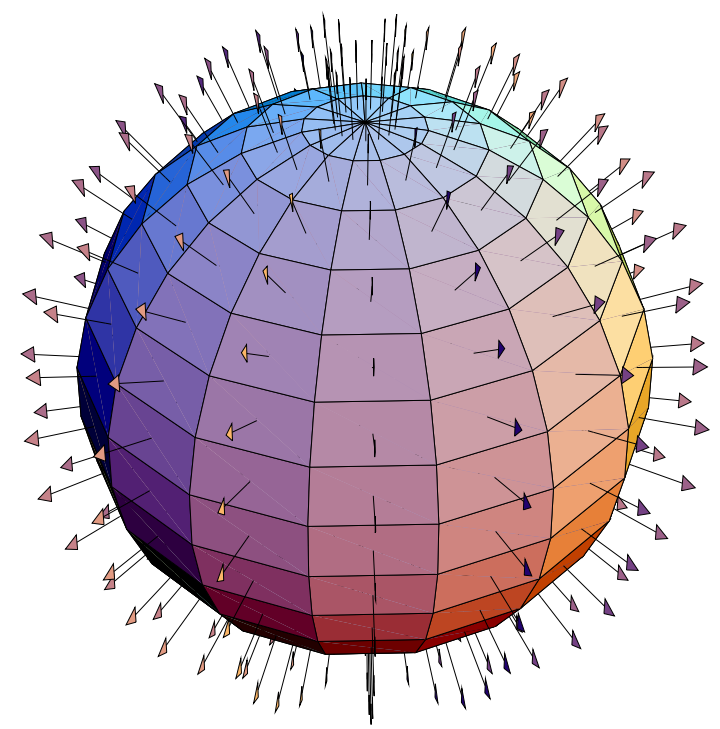

Fig. 1. Artistic impression (by T. Gisiger) of the field of the Skyrmion or hedgehog. But topologically

$$
\mathbb{R}^{3}+\infty=S^{3}
$$

thus the homotopy classes of mappings

$$
U(\vec{x}): S^{3} \rightarrow S^{3}
$$

which define $\Pi_{3}\left(S^{3}\right)=\mathbb{Z}$, characterize the space of configurations. The topological charge of each sector is given by

$$
N=\frac{1}{24 \pi^{2}} \int d^{3} \vec{x} \epsilon^{\mathrm{ijk}} \operatorname{tr}\left(U^{\dagger} \partial_{\mathrm{i}} U U^{\dagger} \partial_{j} U U^{\dagger} \partial_{\mathrm{k}} U\right)
$$

which is an integer and is identified with the baryon number [1, [3].

The solution with baryon number $N=1$ with lowest energy is the Skyrmion. It is parametrized presumably (since this has still not been rigorously, mathematically proven) by the following expression

$$
U(\vec{x})=\mathrm{e}^{\mathrm{i} \hat{r} \cdot \vec{\tau} f(r)}
$$

where $\vec{\tau}$ are the Pauli matrices, $\hat{r}$ is the unit position vector and $r$ is its length. The field $U(\vec{x})$ therefore points radially, and is sometimes called "hedgehog" (see figure 1). $f(r)$ is a function to be obtained from the equations of motion 
of the theory by replacing the ansatz (10) in the static equations of the theory:

$$
\partial_{\mathrm{k}}\left(\frac{f_{\pi}^{2}}{2} \partial_{\mathrm{k}} U U^{\dagger}+\frac{1}{8 e^{2}}\left[\partial_{\mathrm{i}} U U^{\dagger},\left[\partial_{\mathrm{k}} U U^{\dagger}, \partial_{\mathrm{i}} U U^{\dagger}\right]\right]\right)=0
$$

which give for (10)

$$
\left(\frac{\rho}{4}+2 \sin ^{2} F\right) F^{\prime \prime}+\frac{1}{2} \rho F^{\prime}+\sin 2 f F^{\prime 2}-\frac{1}{4} \sin 2 F-\frac{\sin ^{2} F \sin 2 F}{\rho^{2}}=0
$$

where $\rho \equiv 2 e f_{\pi} r$ and $f(r)=F(\rho)$. This equation has to be solved numerically using the boundary conditions imposed on the ansatz by the requirements that it have baryon number unity and finite energy, namely $f(0)=\pi$ and $f(+\infty)=0$. The asympotic behaviour of $f(r)$ for large values of $r$ is obtainable from (12):

$$
f(r) \rightarrow \frac{\kappa}{r^{2}}
$$

Using numerical integration we find that $\kappa \simeq 2.16 / e^{2} f_{\pi}^{2}$,9,41] (The equation for $f(r)$ can equally well be obtained by replacing the ansatz (10) in the energy functional (5), extremizing relative to $f(r)$, and integrating over $\theta$ and $\phi$ ).

Using the definitions

$$
\begin{aligned}
& \mathcal{R}_{\mu}^{\mathrm{a}}(U)=-\frac{\mathrm{i}}{2} \operatorname{tr}\left[\tau^{\mathrm{a}} \partial_{\mu} U U^{\dagger}\right] \\
& \mathcal{L}_{\mu}^{\mathrm{a}}(U)=-\frac{\mathrm{i}}{2} \operatorname{tr}\left[\tau^{\mathrm{a}} U^{\dagger} \partial_{\mu} U\right] \\
& D_{\mathrm{ab}}(U)=\frac{1}{2} \operatorname{tr}\left[\tau^{\mathrm{a}} U \tau^{\mathrm{b}} U^{\dagger}\right]
\end{aligned}
$$

the Skyrme Lagrange density can be written in the following fashion

$$
\begin{aligned}
\mathcal{L}_{\text {sk }}=\frac{f_{\pi}^{2}}{2} \mathcal{R}(U)_{\mu} \cdot \mathcal{R}(U)^{\mu}-\frac{1}{4 e^{2}}[ & \mathcal{R}(U)_{\mu} \cdot \mathcal{R}(U)^{\mu} \mathcal{R}(U)_{\nu} \cdot \mathcal{R}(U)^{\nu} \\
& \left.-\mathcal{R}(U)_{\mu} \cdot \mathcal{R}(U)^{\nu} \mathcal{R}(U)_{\nu} \cdot \mathcal{R}(U)^{\mu}\right]
\end{aligned}
$$

where the "." represents, for example, $\mathcal{R}_{\mu} \cdot \mathcal{R}^{\mu}=\mathcal{R}_{\mu}^{\mathrm{a}} \mathcal{R}^{\mathrm{a} \mu}$. We can separate this into a kinetic energy $\mathcal{T}$ which is the part quadratic in time derivatives and a potential energy $\mathcal{V}$ without any time derivatives:

$$
\mathcal{T}=\frac{f_{\pi}^{2}}{2} \mathcal{R}_{0} \cdot \mathcal{R}_{0}-\frac{1}{2 e^{2}}\left[\mathcal{R}_{0} \cdot \mathcal{R}_{\mathrm{i}} \mathcal{R}_{0} \cdot \mathcal{R}_{\mathrm{i}}-\mathcal{R}_{0} \cdot \mathcal{R}_{0} \mathcal{R}_{\mathrm{i}} \cdot \mathcal{R}_{\mathrm{i}}\right]
$$




$$
\mathcal{V}=-\frac{f_{\pi}^{2}}{2} \mathcal{R}_{\mathrm{i}} \cdot \mathcal{R}_{\mathrm{i}}-\frac{1}{4 e^{2}}\left[\mathcal{R}_{\mathrm{i}} \cdot \mathcal{R}_{\mathrm{i}} \mathcal{R}_{\mathrm{j}} \cdot \mathcal{R}_{\mathrm{j}}-\mathcal{R}_{\mathrm{i}} \cdot \mathcal{R}_{\mathrm{j}} \mathcal{R}_{\mathrm{j}} \cdot \mathcal{R}_{\mathrm{i}}\right]
$$

The parametrization of a Skyrmion with (iso)orientation defined by a time dependent $\mathrm{SU}(2)$ matrix $A(t)$ and position $\vec{R}(t)$ is

$$
U(\vec{x}, t)=A(t) U(\vec{x}-\vec{R}(t)) A(t)^{\dagger}
$$

This gives a Skyrmion 6 degrees of freedom. After replacing this ansatz in the Skyrme Lagrangian and integrating over all space, we find [4]:

$$
L=-2 M+\frac{1}{2} M \dot{\vec{R}}^{2}+2 \Lambda\left(\mathcal{R}_{0}^{\mathrm{a}}(A) \mathcal{R}_{0}^{\mathrm{a}}(A)\right)
$$

where

$$
\begin{aligned}
M & =4 \pi \int_{0}^{+\infty} r^{2} d r \\
& \times\left\{\frac{1}{8} f_{\pi}^{2}\left[\left(\frac{\partial f}{\partial r}\right)^{2}+2 \frac{\sin ^{2} f}{r^{2}}\right]+\frac{1}{2 e^{2}} \frac{\sin ^{2} f}{r^{2}}\left[\frac{\sin ^{2} f}{r^{2}}+2\left(\frac{\partial f}{\partial r}\right)^{2}\right]\right\}
\end{aligned}
$$

is the mass of a Skyrmion and (see for instance [20])

$$
\Lambda=\left(e f_{\pi}\right)^{3} \int r^{2} d r \sin ^{2} f\left[1+\frac{4}{\left(e f_{\pi}\right)^{2}}\left(f^{\prime 2}+\frac{\sin ^{2} f}{r^{2}}\right)\right]
$$

is its moment of inertia. This roughly gives the Skyrmion a mass of $850 \mathrm{MeV}$ (quite close to the nucleon mass), or in natural units of energy $\simeq 1.23 \times$ $12 \pi^{2}[28]$. The moment of inertia is roughly equal to $(1 / 195) \mathrm{MeV}^{-1}$ [20].

\section{Study of the statics of the Skyrme model}

We now start the first part of this review which is devoted to the study of the static sector of the model. Most of the material concerns geometric aspects of the model, but a few pages are devoted to the instanton method, rational maps and Morse theory. 


\subsection{Geometry of the Skyrme model}

In this subsection we will first briefly review the theory of non-linear deformations of a body. Most of the material comes from Ogden [42] and readily generalizes to the case of a field theory. We then take up the case of the Skyrme model defined on various spatial manifolds, presenting the findings of Manton[28] and Manton and Ruback[27].

\subsubsection{Non-linear deformation of a body}

Let us consider a body $B$ at rest, i.e., free of oscillations or interior motion: the action of exterior forces (like gravity) if any, and that of the interior forces caused by the nature of the body (the interactions between the atoms of the body, for instance) exactly cancel each other. We will call this state of the body its initial configuration. Let us now change the shape of the body by applying forces to it, until it reaches a new static configuration. Then it is possible to characterize this action by a function $\vec{\chi}$ which we now define. Let $\vec{X}$ be the initial position of a given point $P$ of the body, and $\vec{x}$ its position in the final configuration. Then we can define the deformation function $\vec{\chi}$ as mapping $\vec{X}$ to $\vec{x}$ for every point of the body:

$$
\begin{aligned}
\vec{\chi}: & \mathbb{R}^{3} \rightarrow \mathbb{R}^{3} \\
& \vec{X} \mapsto \vec{x}=\vec{\chi}(\vec{X}) .
\end{aligned}
$$

For our needs, we will restrict ourselves to $\vec{\chi}$ being continuous and twice differentiable. In the case of a real dynamical system, $\vec{\chi}$ could depend on time but here we will only consider static configurations. See Figure 2 for an example of deformation parametrized by a function $\vec{\chi}$.

To make things more concrete, let us introduce coordinate systems in the initial and final configurations. Using two different coordinate systems to describe the deformation of a body can seem like overkill but we follow this course for two reasons. The first is that it enables one to choose coordinate systems which best suit each configuration, simplifying the computations that follow (which are usually quite complex in real life problems). The second, more important reason is that it will make the jump to Manton's elasticity formalism in the frame of field theory easier and more natural. We note that we will only be working with bases of vectors which are locally orthogonal, and that most of the following equations are written in cartesian coordinates. All tensorial equations are readily generalized to arbitrary curvilinear systems.

Let $O$ be the origin of the system in the initial configuration and $\left\{\vec{E}_{\mathrm{m}}\right\}$ its base. Following the convention used by Manton[28], we will use the indices 

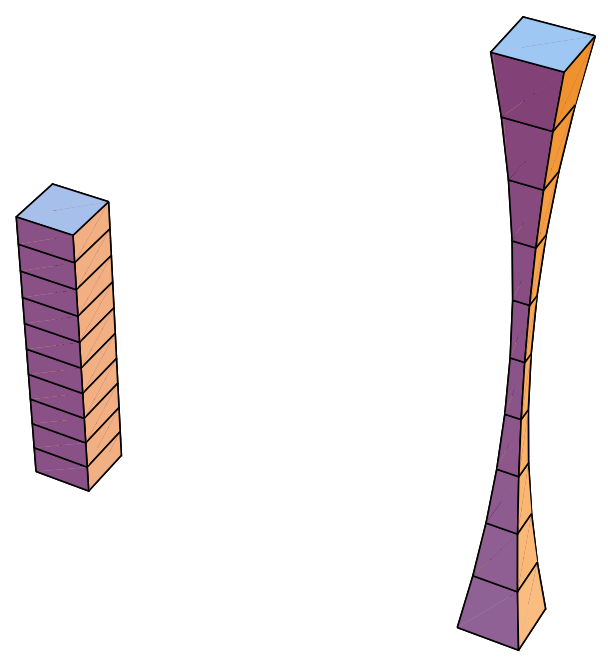

Fig. 2. Example of deformation of a solid (left) before the deformation and (right) after a simple stretching along the $z$ axis.

$m, n, o, p$ to denote basis vectors. The position $\vec{X}$ of a point of the body is defined by

$$
\begin{aligned}
\vec{X} & =\left(\vec{X} \cdot \vec{E}_{\mathrm{m}}\right) \vec{E}_{\mathrm{m}} \\
& \equiv X^{\mathrm{m}} \vec{E}_{\mathrm{m}}
\end{aligned}
$$

where summation over $m$ is implicit. $\vec{X}$ then has coordinates $X^{\mathrm{m}}$ in this coordinate system.

Let $o$ and $\left\{\vec{\xi}_{\mu}\right\}$ be the origin and base of the coordinate system we choose to describe the final configuration, respectively (We will use the indices $\mu, \nu \rho, \sigma$ here). Then we express $\vec{x}$ in the final basis as

$$
\begin{aligned}
\vec{x} & =\left(\vec{x} \cdot \vec{\xi}_{\mu}\right) \vec{\xi}_{\mu} \\
& \equiv x^{\mu} \vec{\xi}_{\mu} .
\end{aligned}
$$

Then equation (24) can be written in terms of the components

$$
x^{\mu}=\chi^{\mu}\left(X^{\mathrm{m}}\right) .
$$

The deformation of the body is completely contained in the map $\vec{\chi}$. How- 
ever the map contains much redundancy which we will isolate next. Under deformation an element of length $\mathrm{d} \vec{X}$ transforms according to

$$
\mathrm{d} \vec{x}=\frac{\partial \vec{\chi}}{\partial \vec{X}} \mathrm{~d} \vec{X}
$$

or in coordinates

$$
\mathrm{d} x^{\mu}=\frac{\partial \chi^{\mu}}{\partial X^{\mathrm{m}}} \mathrm{d} X^{\mathrm{m}}
$$

Let us introduce the second order tensor

$$
A=A_{\mu \mathrm{m}} \vec{\xi}_{\mu} \otimes \vec{E}_{\mathrm{m}}
$$

with

$$
A_{\mu \mathrm{m}}=\frac{\partial \chi^{\mu}}{\partial X^{\mathrm{m}}}
$$

which is called the deformation gradient (relative to the reference configuration). This is just the local Jacobian of the deformation defined by (24).

Intuitively it is clear that $A$ completely represents the deformation, but as we shall demonstrate, it is also sensitive to the choices of the bases $\left\{\vec{E}_{\mathrm{m}}\right\}$ and $\left\{\vec{\xi}_{\mu}\right\}$ which have no physical content. For instance, a physical quantity such as the amount of energy stored in the body by the deformation should not depend on these choices.

Let us consider new bases $\left\{\vec{E}_{\mathrm{m}}^{\prime}\right\}$ and $\left\{\vec{\xi}_{\mu}^{\prime}\right\}$ defined by

$$
\begin{aligned}
& \vec{E}_{\mathrm{m}}^{\prime}=\Omega_{\mathrm{mn}} \vec{E}_{\mathrm{n}} \\
& \vec{\xi}_{\mu}^{\prime}=W_{\mu \nu} \vec{\xi}_{\nu}
\end{aligned}
$$

where $\Omega$ and $W$ are orthogonal matrices. The new bases correspond to a new choice of orientation for the bases in the initial and final configuration. The tensor $A$ expresses itself in each basis as follows:

$$
\begin{aligned}
A & =A_{\mu \mathrm{m}} \vec{\xi}_{\mu} \otimes \vec{E}_{\mathrm{m}} \\
& =A_{\nu \mathrm{n}}^{\prime} \vec{\xi}_{\nu}^{\prime} \otimes \vec{E}_{\mathrm{n}}^{\prime}
\end{aligned}
$$

where

$$
A_{\nu \mathrm{n}}^{\prime}=W_{\nu \mu} A_{\mu \mathrm{m}}\left(\Omega^{\mathrm{T}}\right)_{\mathrm{mn}}
$$


and $\Omega^{\mathrm{T}}$ is the transpose of $\Omega$. Therefore $A$ depends strongly on the choice of the bases. In the normal theory of elasticity, $A$ is considered to be non singular: no $\mathrm{d} \vec{X}$ can be deformed into a line element $\mathrm{d} \vec{x}$ with zero length. Such an annihilation of a line element would imply an infinite force acting on the body, which is unrealistic. But in the next subsections, devoted to the study of the Skyrme model as a theory of elasticity, we will see that there is physical meaning to a singular or in fact zero tensor $A$.

A step toward a better description of the deformation is to use the right Cauchy-Green deformation tensor $D=A A^{\mathrm{T}}$. It is symmetric and positive definite by construction and under the change of coordinate axes mentioned above, $D \rightarrow D^{\prime}=W D W^{\mathrm{T}}$ which is physically more sound but still sensitive to the choice of the coordinate system of the final configuration.

The right Cauchy-Green tensor is related in a simple way to the usual strain tensor of elasticity. To show this let us define the displacement vector $\vec{u}$ :

$$
\vec{u}(\vec{X})=\vec{x}-\vec{X}=\vec{\chi}(\vec{X})-\vec{X}
$$

which measures how much a point moves during the deformation. It is usually assumed to be very small in the ordinary, i.e. linear, theory of elasticity. Define $G$ the displacement gradient

$$
G_{\mu \mathrm{m}}=(A-\mathrm{I})_{\mu \mathrm{m}}=\frac{\partial \mathrm{u}^{\mu}}{\partial \mathrm{X}^{\mathrm{m}}}
$$

Then one can define two tensors of strain:

$$
\begin{aligned}
E & =\frac{1}{2}\left(G+G^{\mathrm{T}}+G^{\mathrm{T}} G\right) \\
& =\frac{1}{2}\left(A A^{\mathrm{T}}-\mathrm{I}\right)=\frac{1}{2}(\mathrm{D}-\mathrm{I})
\end{aligned}
$$

and

$$
\begin{aligned}
F & =\frac{1}{2}\left(G+G^{\mathrm{T}}+G G^{\mathrm{T}}\right) \\
& =\frac{1}{2}\left(A^{\mathrm{T}} A-\mathrm{I}\right)
\end{aligned}
$$

In component formulation this gives :

$$
E_{\mu \mathrm{m}}=\frac{1}{2}\left(\frac{\partial u^{\mu}}{\partial X^{\mathrm{m}}}+\frac{\partial u^{\mathrm{m}}}{\partial X^{\mu}}+\sum_{\mathrm{n}=1}^{3} \frac{\partial u^{\mathrm{n}}}{\partial X^{\mu}} \frac{\partial u^{\mathrm{n}}}{\partial X^{\mathrm{m}}}\right)
$$


and

$$
F_{\mu \mathrm{m}}=\frac{1}{2}\left(\frac{\partial u^{\mu}}{\partial X^{\mathrm{m}}}+\frac{\partial u^{\mathrm{m}}}{\partial X^{\mu}}+\sum_{\mathrm{n}=1}^{3} \frac{\partial u^{\mu}}{\partial X^{\mathrm{n}}} \frac{\partial u^{\mathrm{m}}}{\partial X^{\mathrm{n}}}\right) .
$$

$E$ is the ordinary strain tensor considered in Ogden 42 while the reader will recognize $F$ to be the strain tensor defined in the theory of elasticity, see for example Landau[43]. Notice that the physical content of $D$ and $E$ coincide since they differ by a translation and a factor, as seen from equation (39).

The non-redundant description of the deformation is furnished by functions of the matrix $D$ which are invariant under conjugation by orthogonal matrices. These functions are given by the secular (determinant) equation of the matrix $D$ :

$$
\operatorname{det}(D-\delta I)=\delta^{3}+I_{1}(D) \delta^{2}+I_{2}(D) \delta+I_{3}(D)=0
$$

One finds

$$
\begin{aligned}
I_{1} & =\operatorname{tr} D \\
I_{2} & =\frac{1}{2}\left[(\operatorname{tr} D)^{2}-\operatorname{tr}\left(D^{2}\right)\right] \\
I_{3} & =\operatorname{det} D .
\end{aligned}
$$

The set of eigenvalues of $D$ is invariant under orthogonal conjugation, as are the coefficients $I_{\mathrm{i}}$, which can be verified easily. The set of invariants $I_{\mathrm{i}}$ is complete since the eingenvalues of $D$ are uniquely determined by equation (42).

These eigenvalues can in fact be expressed in terms of the eigenvalues of a matrix obtained from $A$. This can be seen via the following analysis. Any non-singular, real matrix $A$ admits a polar decomposition

$$
\begin{aligned}
A & =R U \\
& =V R
\end{aligned}
$$

where $R$ is an orthogonal matrix and $U$ and $V$ are symmetric, non-singular, real matrices 42,44. Let $\vec{u}_{\mathrm{i}}$ and $\lambda_{\mathrm{i}}$ denote the eigenvectors and corresponding eigenvalues of $U$

$$
U \vec{u}_{\mathrm{i}}=\lambda_{\mathrm{i}} \vec{u}_{\mathrm{i}} \quad i=1,2,3
$$


where the $\vec{u}_{\mathrm{i}}$ can be chosen orthonormal since $U$ is a symmetric, real (hermitian) matrix. Then

$$
A \vec{u}_{\mathrm{i}}=R U \vec{u}_{\mathrm{i}}=\lambda_{\mathrm{i}}\left(R \vec{u}_{\mathrm{i}}\right)=V\left(R \vec{u}_{\mathrm{i}}\right)
$$

hence $R \vec{u}_{\mathrm{i}}$ is an eigenvector of $V$ with the same eigenvalue $\lambda_{\mathrm{i}}$.

Now

$$
D=A A^{\mathrm{T}}=V^{2}=R U^{2} R^{\mathrm{T}}
$$

thus

$$
D\left(R \vec{u}_{\mathrm{i}}\right)=\lambda_{\mathrm{i}}^{2}\left(R \vec{u}_{\mathrm{i}}\right)
$$

and the three invariants (43), (44) and (45) are

$$
\begin{aligned}
& I_{1}=\lambda_{1}^{2}+\lambda_{2}^{2}+\lambda_{3}^{2} \\
& I_{2}=\lambda_{1}^{2} \lambda_{2}^{2}+\lambda_{2}^{2} \lambda_{3}^{2}+\lambda_{3}^{2} \lambda_{1}^{2} \\
& I_{3}=\lambda_{1}^{2} \lambda_{2}^{2} \lambda_{3}^{2} .
\end{aligned}
$$

These invariants will be useful in writing the energy stored in the body due to the deformation. The same applies to the Skyrme model, as we will show below. We can give a physical interpretation of the three invariants in terms of stretching of a set of vectors and various associated geometrical quantities.

The simplest invariant is $I_{3}$ which is related to the change in the volume defined by three non-coplanar vectors $\mathrm{d} \vec{X}^{(1)}, \mathrm{d} \vec{X}^{(2)}$ and $\mathrm{d} \vec{X}^{(3)}$, and their images $\mathrm{d} \vec{x}^{(1)}, \mathrm{d} \vec{x}^{(2)}$ and $\mathrm{d} \vec{x}^{(3)}$ under the deformation. By equation (28), we have

$$
\mathrm{d} \vec{x}=A \cdot \mathrm{d} \vec{X}
$$

which gives

$$
\mathrm{d} v=\operatorname{det} A \mathrm{~d} V=\lambda_{1} \lambda_{2} \lambda_{3} \mathrm{~d} V
$$

where

$$
\begin{aligned}
\mathrm{d} V & =\mathrm{d} \vec{X}^{(1)} \cdot \mathrm{d} \vec{X}^{(2)} \times \mathrm{d} \vec{X}^{(3)} \\
\mathrm{d} v & =\mathrm{d} \vec{x}^{(1)} \cdot \mathrm{d} \vec{x}^{(2)} \times \mathrm{d} \vec{x}^{(3)}
\end{aligned}
$$


assuming that $\mathrm{d} \vec{x}^{(\mathrm{i})}$ and $\mathrm{d} \vec{X}^{(\mathrm{i})}$ form right handed triads. So the third invariant is just the square of the change of a volume under the deformation.

Let us define the stretch of a line element of the body as measured by the ratio between the length of the initial line element and that of its image under the deformation. From (28) and (31) we have

$$
\hat{m}|\mathrm{~d} \vec{x}|=A \hat{M}|\mathrm{~d} \vec{X}|
$$

where $\hat{m}, \hat{M}$ are unit vectors along the direction of $\mathrm{d} \vec{x}$ and $\mathrm{d} \vec{X}$ and $|\ldots|$ indicates the length. Taking the norm of both sides of (59) we define

$$
\lambda(\hat{M}) \equiv \frac{|\mathrm{d} \vec{x}|}{|\mathrm{d} \vec{X}|}=\sqrt{\hat{M} \cdot A^{\mathrm{T}} A \cdot \hat{M}}
$$

which gives the stretch in the direction $\hat{M}$ at $\vec{X}$. Taking $\hat{M}$ to be colinear to the $n$-th eigenvector $\hat{u}_{\mathrm{n}}$ of $A^{\mathrm{T}} A$ with eigenvalue $\lambda_{\mathrm{n}}^{2}$, we get

$$
\lambda^{2}\left(\hat{u}_{\mathrm{n}}\right)=\lambda_{\mathrm{n}}^{2}
$$

The first invariant $I_{1}$ is then just the sum of the squares of the stretch along the three eigenvectors of $U$. These eigenvectors correspond exactly to the usual principal directions of strain. Indeed from (39) we see that the matrix $F$ corresponds to the usual definition of the strain tensor. However

$$
F=\frac{1}{2}\left(U^{2}-1\right)
$$

hence

$$
F \vec{u}_{\mathrm{i}}=\frac{1}{2}\left(\lambda_{\mathrm{i}}^{2}-1\right) \vec{u}_{\mathrm{i}}
$$

thus showing that the $\vec{u}_{\mathrm{i}}$ correspond to the principal directions of strain.

The second invariant is the most subtle and is related to the change under the deformation of the area elements defined by an orthonormal triad. An orthonormal triad $\hat{v}_{\mathrm{i}}$ defines the three area elements

$$
\frac{1}{2} \epsilon_{\mathrm{ijk}} \hat{v}_{\mathrm{j}} \times \hat{v}_{\mathrm{k}} \quad i, j, k=1,2,3 .
$$

Under the deformation these are transformed to

$$
\frac{1}{2} \epsilon_{\mathrm{ijk}}\left(A \hat{v}_{\mathrm{j}}\right) \times\left(A \hat{v}_{\mathrm{k}}\right) \text {. }
$$


Computing the norm and summing over $i$ yields

$$
\begin{aligned}
& \frac{1}{4} \epsilon_{\mathrm{ijk}} \epsilon_{\mathrm{ilm}}\left(A \hat{v}_{\mathrm{j}} \times A \hat{v}_{\mathrm{k}}\right) \cdot\left(A \hat{v}_{\mathrm{l}} \times A \hat{v}_{\mathrm{m}}\right) \\
= & \frac{1}{2}\left(A \hat{v}_{\mathrm{j}} \times A \hat{v}_{\mathrm{k}}\right) \cdot\left(A \hat{v}_{\mathrm{j}} \times A \hat{v}_{\mathrm{k}}\right) \\
= & \frac{1}{2}\left[\left(A \hat{v}_{\mathrm{j}} \cdot A \hat{v}_{\mathrm{j}}\right)\left(A \hat{v}_{\mathrm{k}} \cdot A \hat{v}_{\mathrm{k}}\right)-\left(A \hat{v}_{\mathrm{j}} \cdot A \hat{v}_{\mathrm{k}}\right)\left(A \hat{v}_{\mathrm{j}} \cdot A \hat{v}_{\mathrm{k}}\right)\right]
\end{aligned}
$$

With

$$
\hat{v}_{\mathrm{i}}=v_{\mathrm{i}}^{\mathrm{k}} \vec{u}_{\mathrm{k}}
$$

where $v_{\mathrm{i}}^{\mathrm{k}}$ are the elements of an orthogonal matrix (since $\hat{v}_{\mathrm{i}}$ are orthonormal), we obtain

$$
A \hat{v}_{\mathrm{i}}=v_{\mathrm{i}}^{\mathrm{k}} A \vec{u}_{\mathrm{k}}=v_{\mathrm{i}}^{\mathrm{k}} \lambda_{\mathrm{k}} R \vec{u}_{\mathrm{k}}
$$

SO

$$
\begin{aligned}
A \hat{v}_{\mathrm{j}} \cdot A \hat{v}_{\mathrm{k}} & =v_{\mathrm{j}}^{\mathrm{l}} v_{\mathrm{k}}^{\mathrm{m}} \lambda_{\mathrm{l}} \lambda_{\mathrm{m}}\left(R \vec{u}_{\mathrm{l}} \cdot R \vec{u}_{\mathrm{m}}\right) \\
& =v_{\mathrm{j}}^{\mathrm{l}} v_{\mathrm{k}}^{\mathrm{l}} \lambda_{\mathrm{l}}^{2}
\end{aligned}
$$

Hence the squared norm is now equal to

$$
\begin{aligned}
& \frac{1}{2}\left(\left(v_{\mathrm{j}}^{\mathrm{l}} v_{\mathrm{j}}^{\mathrm{l}} \lambda_{\mathrm{l}}^{2}\right)\left(v_{\mathrm{k}}^{\mathrm{m}} v_{\mathrm{k}}^{\mathrm{m}} \lambda_{\mathrm{m}}^{2}\right)-\left(v_{\mathrm{j}} v_{\mathrm{k}}^{\mathrm{l}} \lambda_{\mathrm{l}}^{2}\right)\left(v_{\mathrm{j}}^{\mathrm{m}} v_{\mathrm{k}}^{\mathrm{m}} \lambda_{\mathrm{m}}^{2}\right)\right) \\
= & \frac{1}{2}\left(\sum_{\mathrm{l}} \lambda_{\mathrm{l}}^{2} \sum_{\mathrm{m}} \lambda_{\mathrm{m}}^{2}-\sum_{\mathrm{l}} \lambda_{\mathrm{l}}^{4}\right) \\
= & \lambda_{1}^{2} \lambda_{2}^{2}+\lambda_{2}^{2} \lambda_{3}^{2}+\lambda_{3}^{2} \lambda_{1}^{2} \\
= & I_{2} .
\end{aligned}
$$

\subsubsection{Geometrical framework for the Skyrme model}

A field corresponds to a mapping $\pi$ from the manifold $S$ of ordinary space to the manifold $\Sigma$ of the target space. A field theory further specifies the dynamics obeyed by the field via the Euler-Lagrange equations obtained from the Lagrangian of the theory. We will limit ourselves to the case where both $S$ and $\Sigma$ are 3 -spheres, the former being a 3 -sphere of radius $L$, while the latter is the 3 -sphere of the $S U(2)$ group corresponding to isospin. The case of the ordinary Skyrmion defined on $\mathbb{R}^{3}$ is obtained by taking $L \rightarrow+\infty$. 
This modest generalization of the Skyrme model allows for a non-trivial application of the geometrical formalism of non-linear elasticity theory which we have just elaborated. Varying the radius $L$ allows us to cover the cases from extreme to zero curvature.

The map $\pi$ describes the Skyrme field, whether it is a group of waves with zero baryon number, a Skyrmion or a heavy nucleus of high baryon number. Even if $\pi$ bears similarity with the deformation map $\vec{\chi}$ of the above elasticity theory, it is different in a fundamental way: it maps a curved space onto another curved space. $\vec{\chi}$ only maps a set of points in $\mathbb{R}^{3}$ onto another set of points in $\mathbb{R}^{3}$. Apart from this fundamental difference, there are many similarities between the treatment of elastic bodies and field theories.

The initial spatial manifold with a given metric comes equipped with a tangent space at each point. The tangent space $T_{\mathrm{p}}(S)$ of $S$ at the point $p$ has a natural basis $\left\{\partial / \partial p^{i}\right\}$. Although linearly independent, these vectors are not necessarily orthonormal. By a linear transformation, we can construct an orthonormal basis $\left\{\hat{e}_{\mathrm{m}}\right\}(m=1,2,3)$ of $T_{\mathrm{p}}(S)$ given by

$$
\hat{e}_{\mathrm{m}}=e_{\mathrm{m}}^{\mathrm{i}} \frac{\partial}{\partial p^{\mathrm{i}}}
$$

where the coefficient of the linear transformation are called the dreibein. Doing the same construction at every point $p$ of $S$ defines an orthonormal frame at each point of $S$. We will follow the same convention as Manton and use indices $i, j, k, l$ with reference to the coordinate basis and $m, n, o, p$ with reference to the orthonormal basis. If the reader has difficulty with these notions, we recommend the references already mentioned [23, 24, 25,26].

With coordinates $\pi^{\alpha}$ on $\Sigma$ and its given metric $\tau^{\alpha \beta}(\alpha, \beta, \delta, \gamma$ coordinate indices and $\mu, \nu, \rho, \sigma$ orthonormal basis indices), consider the image of the orthonormal frame field $\left\{\hat{e}_{\mathrm{m}}\right\}$ under $\pi$. We let $\pi^{\alpha}=\pi^{\alpha}\left(p^{\mathrm{i}}\right)$ be the coordinates of the image of $p^{\mathrm{i}}$ for efficiency of notation. According to the transformation law of a vector

$$
e_{\mathrm{m}}^{\mathrm{i}} \rightarrow e_{\mathrm{m}}^{\mathrm{i}} \frac{\partial \pi^{\alpha}}{\partial p^{\mathrm{i}}}
$$

It is evident that the lengths and directions of the orthonormal triad are changed: it is generally no longer orthonormal. As long as the Jacobian of the transformation

$$
J_{\mathrm{i}}^{\alpha}=\frac{\partial \pi^{\alpha}}{\partial p^{\mathrm{i}}}
$$

is non-singular, the image triad defines a basis of the image tangent space. This 
is the generic case but not at all the relevant one in many physical situations, as we will see below.

The (inverse) metric of space $S$ is given by

$$
t^{\mathrm{ij}}=\delta^{\mathrm{mn}} e_{\mathrm{m}}^{\mathrm{i}} e_{\mathrm{n}}^{\mathrm{j}}
$$

where $\delta^{\mathrm{mn}}$ is the usual Kronecker delta. Its image under $\pi$ is

$$
t^{\prime \alpha \beta}=\delta^{\mathrm{mn}} e_{\mathrm{m}}^{\mathrm{i}} \frac{\partial \pi^{\alpha}}{\partial p^{\mathrm{i}}} e_{\mathrm{n}}^{\mathrm{j}} \frac{\partial \pi^{\beta}}{\partial p^{\mathrm{j}}} .
$$

As we will see later the degree to which $t^{\prime \alpha \beta}$ differs from the intrinsic metric $\tau^{\alpha \beta}$ already existant on $\Sigma$, is a measure of the lack of isometricity of the map $\pi$ and the general energy functional for Skyrme type models measures this nonisometricity (an isometry is a map which preserves the metric i.e. distances are left unchanged by the map). Intuitively the mapping $\pi$ produces a strain and the energy functional is a measure of the energy attributed to this strain. If

$$
t^{\prime \alpha \beta}=\tau^{\alpha \beta}
$$

at every point of $\Sigma$ then the map is an isometry.

There are in fact four ways of testing whether or not the map is an isometry, in the event $\pi$ is invertible. In the following paragraph we will make precise the notions of push-forward, pull-back of the inverse metrics and metrics respectively of the spaces $S$ and $\Sigma$.

Any map $\pi$ between manifolds $S$ and $\Sigma$ defines a map $\pi^{*}$ called the pushforward between the corresponding tangent spaces and $\pi_{*}$ called the pull-back between their dual spaces (see figure 3). We remind the reader that the dual space, sometimes called the space of differential forms, is simply the space of real valued linear functions of the tangent space. This means if $v \in T_{\mathrm{p}}$ and $\omega \in T_{\mathrm{p}}^{*}$ ( $T_{\mathrm{p}}^{*}$ is just notation for the dual space), $\omega$ is a linear function taking $v$ to $\mathbb{R}$, and we write $\langle\omega, v\rangle \in \mathbb{R}$ which is also called the contraction of $\omega$ with $v$. The mapping $\pi$ is defined as follows:

$$
\begin{aligned}
\pi: S & \rightarrow \Sigma \\
p & \mapsto \pi(p) .
\end{aligned}
$$

The push-forward $\pi^{*}$ is defined by

$$
\pi^{*}: T_{\mathrm{p}}(S) \rightarrow T_{\pi(\mathrm{p})}(\Sigma)
$$




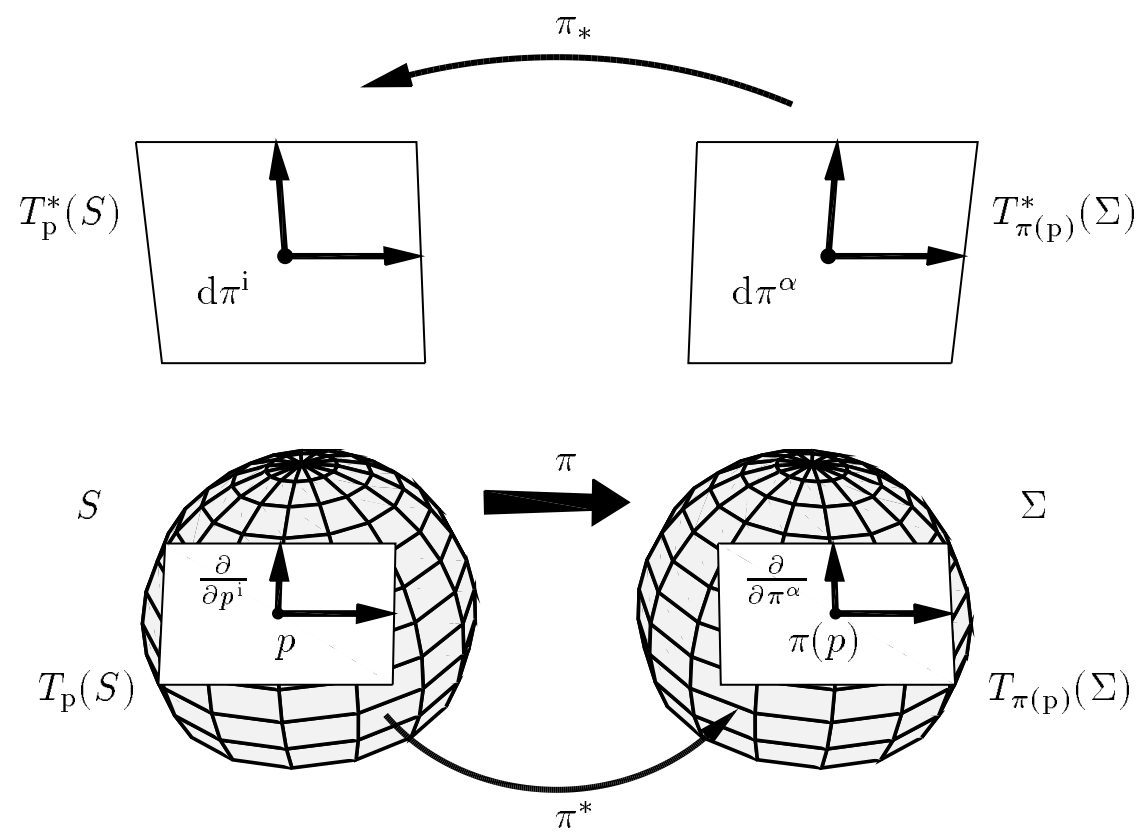

Fig. 3. Diagram of the push-forward and pull-back for the mapping $\pi: S \rightarrow \Sigma$. The same construction is possible for the inverse mapping $\pi^{-1}: \Sigma \rightarrow S$.

where $T_{\mathrm{p}}(S)$ is the tangent space at the point $p$ to the manifold $S$ while $T_{\pi(\mathrm{p})}(\Sigma)$ is the tangent space at the point $\pi(p)$ to the manifold $\Sigma$. A vector $v \in T_{\mathrm{p}}(S)$ with components $v^{\mathrm{i}}$ with respect to the coordinate basis $\left\{\partial / \partial p^{\mathrm{i}}\right\}$

$$
v=v^{\mathrm{i}} \frac{\partial}{\partial p^{\mathrm{i}}}
$$

is mapped to the vector $\pi^{*}(v)$ on $\Sigma$ given in terms of the coordinate basis $\left\{\partial / \partial \pi^{\alpha}\right\}$

$$
\pi^{*}(v)=v^{\mathrm{i}} \frac{\partial \pi^{\alpha}}{\partial p^{\mathrm{i}}} \frac{\partial}{\partial \pi^{\alpha}}
$$

hence the components transform as

$$
v^{\mathrm{i}} \rightarrow \frac{\partial \pi^{\alpha}}{\partial p^{\mathrm{i}}} v^{\mathrm{i}}
$$

The pull-back $\pi_{*}$ is defined as follows:

$$
\pi_{*}: T_{\pi(\mathrm{p})}^{*}(\Sigma) \rightarrow T_{\mathrm{p}}^{*}(S)
$$


where $T_{\pi(\mathrm{p})}^{*}(\Sigma)$ is the dual space to $T_{\pi(\mathrm{p})}(\Sigma)$ i.e. the space of differential 1forms, while $T_{\mathrm{p}}^{*}(S)$ is dual to $T_{\mathrm{p}}(S)$. We remind the reader that the coordinate basis of the cotangent space is defined with respect to the coordinate basis of the tangent space by the condition $\left\langle\mathrm{d} \pi^{\alpha}, \partial / \partial \pi^{\beta}\right\rangle=\delta_{\beta}^{\alpha}$. A 1 -form $\omega \in T_{\pi(\mathrm{p})}^{*}(\Sigma)$ with components $\omega_{\alpha}$ in the dual coordinate basis $\left\{\mathrm{d} \pi^{\alpha}\right\}$,

$$
\omega=\omega_{\alpha} \mathrm{d} \pi^{\alpha}
$$

is mapped to the 1 -form $\pi_{*}(\omega)$ of $T_{\mathrm{p}}^{*}(S)$ with dual coordinate basis $\left\{\mathrm{d} p^{\mathrm{i}}\right\}$

$$
\pi_{*}(\omega)=\omega_{\alpha} \frac{\partial \pi^{\alpha}}{\partial p^{\mathrm{i}}} \mathrm{d} p^{\mathrm{i}}
$$

hence the components transform as

$$
\omega_{\alpha} \rightarrow \omega_{\alpha} \frac{\partial \pi^{\alpha}}{\partial p^{\mathrm{i}}}
$$

These transformation rules generalize tensorially on tensor products of the tangent and dual tangent spaces. Evidently, if $\pi$ is invertible then

$$
\pi^{-1}: \Sigma \rightarrow S
$$

defines a push-forward and a pull-back in the opposite sense. We now come to the point of computing images of the metric and the inverse metric under $\pi$ and under its inverse (if it exists).

Starting with the metric $t$ on $S$ and $\tau$ on $\Sigma$ we have

$$
\begin{aligned}
t & =t_{\mathrm{ij}} \mathrm{d} p^{\mathrm{i}} \otimes \mathrm{d} p^{\mathrm{j}} \\
\tau & =\tau_{\alpha \beta} \mathrm{d} \pi^{\alpha} \otimes \mathrm{d} \pi^{\beta}
\end{aligned}
$$

the corresponding pull-backs via $\pi \equiv \pi(p)$ for $\tau$ and $\pi^{-1} \equiv p(\pi)$ for $t$ are

$$
\tau^{\prime}=\tau_{\alpha \beta} \frac{\partial \pi^{\alpha}}{\partial p^{\mathrm{i}}} \frac{\partial \pi^{\beta}}{\partial p^{\mathrm{j}}} \mathrm{d} p^{\mathrm{i}} \otimes \mathrm{d} p^{\mathrm{j}} \in T_{\mathrm{p}}^{*}(S) \otimes T_{\mathrm{p}}^{*}(S)
$$

and

$$
t^{\prime}=t_{\mathrm{ij}} \frac{\partial p^{\mathrm{i}}}{\partial \pi^{\alpha}} \frac{\partial p^{\mathrm{j}}}{\partial \pi^{\beta}} \mathrm{d} \pi^{\alpha} \otimes \mathrm{d} \pi^{\beta} \in T_{\pi(\mathrm{p})}^{*}(\Sigma) \otimes T_{\pi(\mathrm{p})}^{*}(\Sigma)
$$


Also for the inverse metrics

$$
\begin{aligned}
& t^{-1}=t^{\mathrm{ij}} \frac{\partial}{\partial p^{\mathrm{i}}} \otimes \frac{\partial}{\partial p^{\mathrm{j}}} \\
& \tau^{-1}=\tau^{\alpha \beta} \frac{\partial}{\partial \pi^{\alpha}} \otimes \frac{\partial}{\partial \pi^{\beta}}
\end{aligned}
$$

we have the corresponding push-forwards via $\pi$ for $t^{-1}$ and $\pi^{-1}$ for $\tau^{-1}$,

$$
\left(t^{-1}\right)^{\prime}=t^{\mathrm{ij}} \frac{\partial \pi^{\alpha}}{\partial p^{\mathrm{i}}} \frac{\partial \pi^{\beta}}{\partial p^{\mathrm{j}}} \frac{\partial}{\partial \pi^{\alpha}} \otimes \frac{\partial}{\partial \pi^{\beta}} \in T_{\pi(\mathrm{p})}(\Sigma) \otimes T_{\pi(\mathrm{p})}(\Sigma)
$$

and

$$
\left(\tau^{-1}\right)^{\prime}=\tau^{\mu \nu} \frac{\partial p^{\mathrm{m}}}{\partial \pi^{\mu}} \frac{\partial p^{\mathrm{n}}}{\partial \pi^{\nu}} \frac{\partial}{\partial p^{\mathrm{m}}} \otimes \frac{\partial}{\partial p^{\mathrm{n}}} \in T_{\mathrm{p}}(S) \otimes T_{\mathrm{p}}(S)
$$

Now the condition that the map is an isometry is given by any of the following statements:

$$
\begin{aligned}
t^{\prime}=\tau & \Leftrightarrow & t_{\mathrm{ij}} \frac{\partial p^{\mathrm{i}}}{\partial \pi^{\alpha}} \frac{\partial p^{\mathrm{j}}}{\partial \pi^{\beta}}=\tau_{\alpha \beta} \\
\tau^{\prime}=t & \Leftrightarrow & \tau_{\alpha \beta} \frac{\partial \pi^{\alpha}}{\partial p^{\mathrm{i}}} \frac{\partial \pi^{\beta}}{\partial p^{\mathrm{j}}}=t_{\mathrm{ij}} \\
\left(t^{-1}\right)^{\prime}=\tau^{-1} & \Leftrightarrow & t^{\mathrm{ij}} \frac{\partial \pi^{\alpha}}{\partial p^{\mathrm{i}}} \frac{\partial \pi^{\beta}}{\partial p^{\mathrm{j}}}=\tau^{\alpha \beta} \\
\left(\tau^{-1}\right)^{\prime}=t^{-1} & \Leftrightarrow & \tau^{\alpha \beta} \frac{\partial p^{\mathrm{i}}}{\partial \pi^{\alpha}} \frac{\partial p^{\mathrm{j}}}{\partial \pi^{\beta}}=t^{\mathrm{ij}}
\end{aligned}
$$

If any one of these equations is true, they are all true. Equations (101) and (104) require that $\pi$ is invertible to make sense. As we will see, it is not necessary for $\pi$ to be invertible, hence (102) and (103) are more fundamental. They are all algebraically identical when $\pi$ is invertible. We take (102) as the fundamental relation imposing (locally at the point $p$ ) isometry, since (102) does not require the inverse mapping to exist. (103) is equally suitable.

We give an explicit example of the preceding formalism with the SU(2) Skyrme field defined on $S^{3}$. We take $S$, the initial spatial manifold, to be a topological and metrical $S^{3}$ of radius $L$. The target manifold is the manifold of the group $\mathrm{SU}(2)$ which also happens to be an $S^{3}$. Group manifolds come equipped with a natural metric, the so-called Haar measure, and this gives a natural radius of one to the target 3-sphere. 
With the Cartesian coordinates $X^{1}, X^{2}, X^{3}, X^{4}$ on $\mathbb{R}^{4}$, we define a 3 -sphere of radius $L$ embedded in $\mathbb{R}^{4}$ by the constraint

$$
\sum_{\mathrm{i}=1}^{3} X^{\mathrm{i}} X^{\mathrm{i}}+\left(X^{4}\right)^{2}=L^{2}
$$

With the relation

$$
x^{\mathrm{i}}=\frac{2 L}{L-X^{4}} X^{\mathrm{i}}
$$

where $i=1,2,3$ we effect the stereographic projection to $\mathbb{R}^{3}$ and obtain the following metric

$$
t=\frac{1}{\left(r^{2} / 4 L^{2}+1\right)^{2}} \sum_{\mathrm{i}=1}^{3} \mathrm{~d} x^{\mathrm{i}} \otimes \mathrm{d} x^{\mathrm{i}}
$$

where $r^{2}=\sum_{\mathrm{i}=1}^{3} x^{\mathrm{i}} x^{\mathrm{i}}$. Thus we see that a stereographic projection is simply a conformal transformation of flat space (i.e. the metric only changes by an overall, space dependent, scaling).

The natural metric on the target manifold is best expressed in terms of the left-invariant 1-forms. These are a natural basis of the co-tangent space of $\mathrm{SU}(2)$. If $\pi^{\alpha}(\alpha=1,2,3)$ is any set of local coordinates on $\mathrm{SU}(2)$, they are defined by the following generalisation of (15):

$$
\mathcal{L}^{\mu}=-\frac{\mathrm{i}}{2} \operatorname{tr}\left(\tau^{\mu} U^{\dagger}(\pi) \frac{\partial}{\partial \pi^{\alpha}} U(\pi)\right) d \pi^{\alpha}
$$

where $\tau^{\mu}$ are the Pauli matrices and $U$ taken for convenience to be in the fundamental representation of $\mathrm{SU}(2)$ (our notation is consistent for this subsection, later we will revert to the original notation of section 2). These are left invariants since under the transformation

$$
U \rightarrow V U
$$

where $V$ is a constant element of $\mathrm{SU}(2), \mathcal{L}^{\mu}$ are invariant. (One can also define right invariant 1-forms by exchanging the role of $U$ and $U^{\dagger}$ as in (14)).

For example let us take the coordinates $\{\vec{\pi}\}$ defined by

$$
U=\sqrt{1-\vec{\pi}^{2}}+\mathrm{i} \vec{\pi} \cdot \vec{\tau}=\pi_{0}+\mathrm{i} \vec{\pi} \cdot \vec{\tau} .
$$


Note that $U$ is a function of $\pi^{1}, \pi^{2}, \pi^{3}$ and as such, is not a covariant expression, in the tensorial sense. Indeed, $\pi^{\alpha}$ are also just coordinates and do not transform tensorially either. Hence the expression for $\mathcal{L}^{\mu}$ in the specific coordinates chosen does not appear as a tensorial expression. We find:

$$
\mathcal{L}^{\mu}=\frac{1}{\sqrt{1-\vec{\pi}^{2}}} \sum_{\alpha=1}^{3}\left(\delta^{\mu \beta}+\pi^{\mu} \pi^{\beta}-\delta^{\mu \beta} \vec{\pi}^{2}+\sum_{\beta=1}^{3} \epsilon^{\mu \gamma \beta} \pi^{\gamma}\right) \mathrm{d} \pi^{\beta}
$$

which are the well known Maurer-Cartan forms 26 written in this coordinate system.

This set of 1-forms is natural since, first of all, at the identity where $\vec{\pi}=\overrightarrow{0}$,

$$
\mathcal{L}^{\mu}=\mathrm{d} \pi^{\mu}
$$

Secondly we can obtain $\mathcal{L}^{\mu}$ at any other point in the group via the pull-back of an appropriate mapping defined using the group multiplication. Consider a general element $V_{0}$ in the group with corresponding coordinates $\vec{\pi}_{\mathrm{V}_{0}}$. The mapping of a neighborhood $\mathcal{V}_{\mathrm{V}_{0}}$ of $\vec{\pi}_{\mathrm{V}_{0}}$ to a neighborhood $\mathcal{V}_{\mathrm{I}}$ of the identity I is furnished by multiplication by $V_{0}^{\dagger}$. If a general element of $\mathcal{V}_{\mathrm{V}_{0}}$ is noted by $V$ with coordinates $\vec{\pi}_{\mathrm{V}}$, and a general element of $\mathcal{V}_{\mathrm{I}}$ is noted by $U$ with coordinates $\vec{\pi}$ the map

$$
\begin{aligned}
& V_{0}^{\dagger}: \mathcal{V}_{\mathrm{V}_{0} \rightarrow \mathcal{V}_{\mathrm{I}}} \\
& \begin{aligned}
V \mapsto U=V_{0}^{\dagger} V & =\left(\sqrt{1-\vec{\pi}_{\mathrm{V}_{0}}^{2}}-\mathrm{i} \vec{\pi}_{\mathrm{V}_{0}} \cdot \vec{\tau}\right)\left(\sqrt{1-\vec{\pi}_{\mathrm{V}}^{2}}-\mathrm{i} \vec{\pi}_{\mathrm{V}} \cdot \vec{\tau}\right) \\
& \equiv \sqrt{1-\vec{\pi}\left(\vec{\pi}_{\mathrm{V}_{0}}\right)^{2}}+\mathrm{i} \vec{\pi}\left(\vec{\pi}_{\mathrm{V}_{0}}\right) \cdot \vec{\tau}
\end{aligned}
\end{aligned}
$$

which gives

$$
\vec{\pi}\left(\vec{\pi}_{\mathrm{V}}\right)=-\sqrt{1-\vec{\pi}_{\mathrm{V}}^{2}} \vec{\pi}_{\mathrm{V}_{0}}+\sqrt{1-\vec{\pi}_{\mathrm{V}_{0}}^{2}} \vec{\pi}_{\mathrm{V}}+\vec{\pi}_{\mathrm{V}_{0}} \times \vec{\pi}_{\mathrm{V}}
$$

This induces the pull-back of $\mathrm{d} \pi^{\mu}=\left(\left.\mathcal{L}_{\alpha}^{\mu}\right|_{\vec{\pi}=\overrightarrow{0}}\right) \mathrm{d} \pi^{\alpha}$, clearly where $\left.\mathcal{L}_{\alpha}^{\mu}\right|_{\vec{\pi}=\overrightarrow{0}}=\delta_{\alpha}^{\mu}$,

$$
\begin{aligned}
V_{0 *}^{\dagger}\left(\left.\mathcal{L}_{\alpha}^{\mu}\right|_{\vec{\pi}=\overrightarrow{0}} ^{\mathrm{d} \pi^{\alpha}}\right) & =\left.\left.\mathcal{L}_{\alpha}^{\mu}\right|_{\vec{\pi}=\overrightarrow{0}} \frac{\partial \pi^{\alpha}}{\partial \pi_{\mathrm{V}}^{\beta}}\right|_{\vec{\pi}_{\mathrm{V}}=\vec{\pi}_{\mathrm{V}_{0}}} \mathrm{~d} \pi_{\mathrm{V}}^{\beta} \\
& =\left.\frac{\partial \pi^{\mu}}{\partial \pi_{\mathrm{V}}^{\beta}}\right|_{\vec{\pi}_{\mathrm{V}}=\vec{\pi}_{\mathrm{V}_{0}}} \mathrm{~d} \pi^{\beta} \\
& =\frac{1}{\sqrt{1-\vec{\pi}_{\mathrm{V}_{0}}^{2}}}\left(\delta^{\mu \beta}+\left(\pi_{\mathrm{V}_{0}}^{\mu} \pi_{\mathrm{V}_{0}}^{\beta}-\vec{\pi}_{\mathrm{V}_{0}}^{2} \delta^{\mu \beta}\right)+\epsilon^{\mu \gamma \beta} \pi_{\mathrm{V}_{0}}^{\gamma}\right) \mathrm{d} \pi_{\mathrm{V}}^{\beta}(11)
\end{aligned}
$$


which is exactly as we had found before in equation (111), with $\vec{\pi}=\vec{\pi}_{\mathrm{V}_{0}}$.

The metric on $S U(2)$ is given by

$$
\tau=\delta_{\mu \nu} \mathcal{L}^{\mu} \otimes \mathcal{L}^{\nu}
$$

which clearly indicates the orthonormality of $\mathcal{L}^{\alpha}$, knowing that $\mathcal{L}_{\mu}^{\alpha}$ are invertible as matrices at each point in the group. A short calculation then shows that this implies the metric

$$
\tau=-\operatorname{tr}\left(U^{\dagger}(\pi) \frac{\partial U(\pi)}{\partial \pi^{\mu}} U^{\dagger}(\pi) \frac{\partial U(\pi)}{\partial \pi^{\nu}}\right) \mathrm{d} \pi^{\mu} \otimes \mathrm{d} \pi^{\nu}
$$

Now we return to our setting where $\pi^{\mu}$ the coordinates on the group 3-sphere are functions of $x^{\mathrm{i}}$ the coordinates on the spatial 3 -sphere, since we consider a mapping (that we call $\pi$ ) between these two spaces. The pull-back of the metric to the spatial $S^{3}$ via the mapping is given by

$$
\begin{aligned}
\tau^{\prime} & =\pi_{*}(\tau)=\tau_{\alpha \beta} \frac{\partial \pi^{\alpha}}{\partial x^{\mathrm{i}}} \frac{\partial \pi^{\beta}}{\partial x^{\mathrm{j}}} \mathrm{d} x^{\mathrm{i}} \otimes \mathrm{d} x^{\mathrm{j}} \\
& =-\operatorname{tr}\left(U^{\dagger}(\pi) \frac{\partial U(\pi)}{\partial \pi^{\alpha}} U^{\dagger}(\pi) \frac{\partial U(\pi)}{\partial \pi^{\beta}}\right) \frac{\partial \pi^{\alpha}}{\partial x^{\mathrm{i}}} \frac{\partial \pi^{\beta}}{\partial x^{\mathrm{j}}} \mathrm{d} x^{\mathrm{i}} \otimes \mathrm{d} x^{\mathrm{j}} \\
& =-\operatorname{tr}\left(U^{\dagger} \frac{\partial U}{\partial x^{\mathrm{i}}} U^{\dagger} \frac{\partial U}{\partial x^{\mathrm{j}}}\right) \mathrm{d} x^{\mathrm{i}} \otimes \mathrm{d} x^{\mathrm{j}} \\
& =\frac{2}{1-\vec{\pi}^{2}} \frac{\partial \pi^{\alpha}}{\partial x^{\mathrm{i}}}\left(\delta^{\alpha \beta}+\left(\pi^{\alpha} \pi^{\beta}-\vec{\pi}^{2} \delta^{\alpha \beta}\right)\right) \frac{\partial \pi^{\beta}}{\partial x^{\mathrm{j}}} \mathrm{d} x^{\mathrm{i}} \otimes \mathrm{d} x^{\mathrm{j}}
\end{aligned}
$$

where the last line is relevant to the coordinate system chosen on the group.

The kinetic term of the Skyrme Lagrangian is obtained by contracting $\tau^{\prime}$ with the inverse metric on the spatial manifold

$$
\begin{aligned}
t^{-1}=(1 & \left.+r^{2} / 4 L^{2}\right)^{2} \delta^{\mathrm{ij}} \frac{\partial}{\partial x^{\mathrm{i}}} \otimes \frac{\partial}{\partial x^{\mathrm{j}}} \\
\left\langle\tau^{\prime}, t^{-1}\right\rangle & =t^{\mathrm{ij}} \frac{\partial \pi^{\alpha}}{\partial p^{\mathrm{i}}} \frac{\partial \pi^{\beta}}{\partial p^{\mathrm{j}}} \tau_{\alpha \beta} \\
& =-\left(1+r^{2} / 4 L^{2}\right)^{2} \sum_{\mathrm{i}=1}^{3} \operatorname{tr}\left(U^{\dagger} \frac{\partial}{\partial x^{\mathrm{i}}} U U^{\dagger} \frac{\partial}{\partial x^{\mathrm{i}}} U\right) .
\end{aligned}
$$

Including the volume measure $\sqrt{g}=1 /\left(1+r^{2} / 4 L^{2}\right)^{3}$

$$
\sqrt{g}\left\langle\tau^{\prime}, t^{-1}\right\rangle=-\frac{1}{1+r^{2} / 4 L^{2}} \sum_{\mathrm{i}=1}^{3} \operatorname{tr}\left(U^{\dagger} \frac{\partial}{\partial x^{\mathrm{i}}} U U^{\dagger} \frac{\partial}{\partial x^{\mathrm{i}}} U\right)
$$


which has the correct limit as $L \rightarrow+\infty$.

\subsubsection{Non-linear elasticity theory on a curved space and the Skyrme model}

Now we make the connection with the non-linear elasticity theory that we have treated previously, except generalized to a curved space. Hence we suppose that $X^{\mathrm{i}}$ are coordinates on a curved space and that the particle $p$ of the body at the point $X_{\mathrm{p}}^{\mathrm{i}}$ is mapped to the point $x_{\mathrm{p}}^{\mathrm{i}}=\chi^{\mathrm{i}}\left(X_{\mathrm{p}}^{\mathrm{j}}\right)$. The metric at the initial point is $t_{\mathrm{ij}}\left(X_{\mathrm{p}}^{\mathrm{k}}\right)$ while at the image point it is $\tau_{\mathrm{ij}}\left(x_{\mathrm{p}}^{\mathrm{k}}\right) \equiv t_{\mathrm{ij}}\left(x^{\mathrm{k}}\right)$. The initial triad is $e_{\mathrm{m}}^{i}\left(X_{\mathrm{p}}^{\mathrm{k}}\right)$ while the final triad is $\xi_{\mathrm{m}}^{\mathrm{i}}\left(x_{\mathrm{p}}^{\mathrm{k}}\right) \equiv e_{\mathrm{m}}^{\mathrm{i}}\left(x_{\mathrm{p}}^{\mathrm{k}}\right)$. The initial orthonormal triad is mapped to the final triad in the following way:

$$
\hat{e}_{\mathrm{m}}=e_{\mathrm{m}}^{\mathrm{i}}\left(X^{\mathrm{k}}\right) \frac{\partial}{\partial X^{\mathrm{i}}} \rightarrow e_{\mathrm{m}}^{\mathrm{i}}\left(X^{\mathrm{k}}\right) \frac{\partial \chi^{\alpha}}{\partial X^{\mathrm{i}}} \frac{\partial}{\partial x^{\alpha}}=\mathrm{e}_{\mathrm{m}}^{\mathrm{i}}\left(X^{\mathrm{k}}\right) \frac{\partial \chi^{\alpha}}{\partial X^{\mathrm{i}}} \xi_{\alpha}^{\mu}\left(x^{\mathrm{k}}\right) \hat{\xi}_{\mu}\left(x^{\mathrm{k}}\right)
$$

where $\xi_{\alpha}^{\mu}\left(x^{\mathrm{k}}\right)$ is the inverse dreibein and $\hat{\xi}_{\mu} \equiv \xi_{\mu}^{\beta} \partial / \partial x^{\beta}$. Hence the analog of the tensor $A_{\mu \mathrm{m}}$ is

$$
\tilde{A}_{\mathrm{m}}^{\mu}=e_{\mathrm{m}}^{\mathrm{j}}\left(X_{\mathrm{p}}^{\mathrm{k}}\right) \frac{\partial \chi^{\alpha}}{\partial X_{\mathrm{p}}^{\mathrm{j}}} \xi_{\alpha}^{\mu}\left(x_{\mathrm{p}}^{\mathrm{k}}\right)
$$

which is the local Jacobian of the deformation.

The right-Cauchy-Green deformation tensor is

$$
D_{\mathrm{mm}}=\tilde{A}_{\mathrm{m}}^{\mathrm{n}} \tilde{A}_{\mathrm{m}}^{\mathrm{n}}
$$

and the ordinary strain tensor is given by

$$
E=\frac{1}{2}(D-I)
$$

The invariants are defined in the same way as before.

Now we go to the completely general situation where we are mapping between two different curved spaces. The deformation matrix now generalizes to

$$
J_{\mathrm{m}}^{\mu}=e_{\mathrm{m}}^{\mathrm{i}} \frac{\partial \pi^{\alpha}}{\partial p^{\mathrm{i}}} \zeta_{\alpha}^{\mu}
$$

where the inverse dreibein $\zeta_{\alpha}^{\mu}$ is defined by the orthonormal basis $\hat{\zeta}_{\mu} \equiv \zeta_{\mu}^{\alpha} \partial / \partial \pi^{\alpha}$ in the space tangent to $\Sigma$, while the equivalent strain tensor is 


$$
\begin{aligned}
D_{\mathrm{mn}} & =\sum_{\mu=1}^{3} J_{\mathrm{m}}^{\mu} J_{\mathrm{n}}^{\mu} \\
& =\sum_{\mu=1}^{3} e_{\mathrm{m}}^{\mathrm{i}} \frac{\partial \pi^{\alpha}}{\partial p^{\mathrm{i}}} \zeta_{\alpha}^{\mu} e_{\mathrm{n}}^{\mathrm{j}} \frac{\partial \pi^{\beta}}{\partial p^{\mathrm{j}}} \zeta_{\beta}^{\mu} \\
& =e_{\mathrm{m}}^{\mathrm{i}} \frac{\partial \pi^{\alpha}}{\partial p^{\mathrm{i}}} \frac{\partial \pi^{\beta}}{\partial p^{\mathrm{j}}} e_{\mathrm{n}}^{\mathrm{j}} \tau_{\alpha \beta} .
\end{aligned}
$$

Finally, taking the first invariant of $D$ by tracing over $m$ and $n$ gives

$$
\begin{aligned}
\operatorname{tr} D & =\sum_{\mathrm{m}=1}^{3} e_{\mathrm{m}}^{\mathrm{i}} \frac{\partial \pi^{\alpha}}{\partial p^{\mathrm{i}}} e_{\mathrm{m}}^{\mathrm{j}} \frac{\partial \pi^{\beta}}{\partial p^{\mathrm{j}}} \tau_{\alpha \beta} \\
& =t^{\mathrm{ij}} \frac{\partial \pi^{\alpha}}{\partial p^{\mathrm{i}}} \frac{\partial \pi^{\beta}}{\partial p^{\mathrm{j}}} \tau_{\alpha \beta} \\
& =-\left(1+r^{2} / 4 L^{2}\right)^{2} \sum_{\mathrm{i}=1}^{3} \operatorname{tr}\left(U^{\dagger} \frac{\partial}{\partial x^{\mathrm{i}}} U U^{\dagger} \frac{\partial}{\partial x^{\mathrm{i}}} U\right) \\
& =\lambda_{1}^{2}+\lambda_{2}^{2}+\lambda_{3}^{2}
\end{aligned}
$$

Equation (128) is clearly the usual kinetic term of the Skyrme model Lagrangian (in the limit where $L \rightarrow+\infty$ ).

The Skyrme term is obtained from the curvature tensor defined on the group manifold, which is pulled back to the space manifold and then contracted twice with the inverse metric there. The curvature tensor is most efficiently defined via the machinery of the exterior algebra and the spin connection. The spin connection is a 1 -form

$$
\omega_{\lambda}^{\nu}=\omega_{\alpha \lambda}^{\nu} \mathrm{d} \pi^{\alpha} \equiv \omega_{\mu \lambda}^{\nu} \mathcal{L}^{\mu}
$$

which satisfies the structure equation

$$
\mathrm{d} \mathcal{L}^{\mu}+\omega_{\lambda}^{\mu} \wedge \mathcal{L}^{\lambda}=0
$$

and the "metricity" condition

$$
\omega_{\nu \lambda}=-\omega_{\lambda \nu}
$$

where $\wedge$ is the wedge or exterior product (which is simply the antisymmetrized tensor product of the forms in question), and $\omega_{\nu \lambda}$ is the spin connection of equation (130) with index lowered by $\delta_{\mu \nu}$. The conditions (130) and (131) are exactly equivalent to the conditions in the usual formulation of differential 
geometry that there is no torsion (the Christoffel symbol is symmetric in its lower two indices) and the metric is covariantly conserved (metricity).

The curvature is then given by the 2 -form

$$
\begin{aligned}
R_{\nu}^{\mu} & =R_{\nu \lambda \rho}^{\mu} \mathcal{L}^{\lambda} \wedge \mathcal{L}^{\rho} \\
& =\mathrm{d} \omega_{\nu}^{\mu}+\omega_{\sigma}^{\mu} \wedge \omega_{\rho}^{\sigma} .
\end{aligned}
$$

The spin connection on the group manifold of the target space $\mathrm{SU}(2)$ is well known 26 and is given by

$$
\omega_{\mu \nu}=\epsilon_{\mu \nu \lambda} \mathcal{L}^{\lambda} \text {. }
$$

Then using the relation

$$
\mathrm{d} \mathcal{L}^{\lambda}=\epsilon_{\mu \nu}^{\lambda} \mathcal{L}^{\mu} \wedge \mathcal{L}^{\nu}
$$

which is a little tedious to verify, a short calculation shows

$$
R_{\nu \lambda \rho}^{\mu}=\epsilon_{\nu \sigma}^{\mu} \epsilon_{\lambda \rho}^{\sigma}
$$

The pull-back of the tensor $R_{\mu \nu \lambda \rho}$ (pull-backs are only defined for co-tangent space tensors) is then

$$
R_{\mathrm{ijkl}}^{\prime}=\frac{\partial \pi^{\alpha}}{\partial p^{\mathrm{i}}} \frac{\partial \pi^{\beta}}{\partial p^{\mathrm{j}}} \frac{\partial \pi^{\gamma}}{\partial p^{\mathrm{k}}} \frac{\partial \pi^{\delta}}{\partial p^{\mathrm{l}}} \mathcal{L}_{\alpha}^{\mu} \mathcal{L}_{\beta}^{\nu} \mathcal{L}_{\gamma}^{\lambda} \mathcal{L}_{\delta}^{\rho} R_{\mu \nu \lambda \rho}
$$

We remark that the derivatives $\partial \pi^{\alpha} / \partial p^{\mathrm{i}}$ etc. serve only to change the variables from group manifold coordinates $\pi^{\alpha}$ to spatial coordinates $p^{\mathrm{i}}$, while the Maurer-Cartan forms contain the essential structure. Application of the following Fierz identities

$$
\begin{aligned}
\tau_{\mathrm{ij}}^{\mathrm{a}} \tau_{\mathrm{kl}}^{\mathrm{b}} \epsilon^{\mathrm{abe}} & =-\frac{1}{2}\left(\tau_{\mathrm{kj}}^{\mathrm{e}} \delta_{\mathrm{il}}+\tau_{\mathrm{il}}^{\mathrm{e}} \delta_{\mathrm{kj}}\right) \\
\tau_{\mathrm{ij}}^{\mathrm{a}} \tau_{\mathrm{kl}}^{\mathrm{a}} & =-\frac{1}{2} \tau_{\mathrm{il}}^{\mathrm{a}} \tau_{\mathrm{kj}}^{\mathrm{a}}+\frac{3}{2} \delta_{\mathrm{il}} \delta_{\mathrm{jk}}
\end{aligned}
$$

yields

$$
R_{\mathrm{ijkl}}^{\prime}=2 \operatorname{tr}\left(\left[U^{\dagger} \partial_{\mathrm{i}} U, U^{\dagger} \partial_{\mathrm{j}} U\right]\left[U^{\dagger} \partial_{\mathrm{k}} U, U^{\dagger} \partial_{\mathrm{l}} U\right]\right)
$$

and contracting twice with the inverse metric on space yields 


$$
\begin{aligned}
R^{\prime} & =t^{\mathrm{ik}} t^{j l} R_{\mathrm{ijkl}}^{\prime} \\
& =\left(1+r^{2} / 4 L^{2}\right)^{4} \delta^{\mathrm{ik}} \delta^{\mathrm{jl}} R_{\mathrm{ijkl}}^{\prime} \\
& =\left(1+r^{2} / 4 L^{2}\right)^{4} \sum_{\mathrm{i}, \mathrm{j}=1}^{3} \operatorname{tr}\left(\left[U^{\dagger} \partial_{\mathrm{i}} U, U^{\dagger} \partial_{\mathrm{j}} U\right]^{2}\right)
\end{aligned}
$$

which is obviously the Skyrme term.

A more geometric and generally valid interpretation is obtained by (for any manifold $S$ and $\Sigma$ ) considering the squared norm of the pull-back of the area element defined by two dual basis vectors in the target space 28. The area element defined by the two dual basis vectors is

$$
\begin{aligned}
A^{\mu \nu} & =\hat{\zeta}^{\mu} \wedge \hat{\zeta}^{\nu}=\zeta_{\alpha}^{\mu} \zeta_{\beta}^{\nu} \mathrm{d} \pi^{\alpha} \wedge \mathrm{d} \pi^{\beta} \\
& =\frac{1}{2}\left(\zeta_{\alpha}^{\mu} \zeta_{\beta}^{\nu}-\zeta_{\beta}^{\mu} \zeta_{\alpha}^{\nu}\right) \mathrm{d} \pi^{\alpha} \wedge \mathrm{d} \pi^{\beta}
\end{aligned}
$$

Its pull-back is given by

$$
\begin{aligned}
A^{\prime \mu \nu} & =A_{\mathrm{ij}}^{\prime \mu \nu} \mathrm{d} p^{\mathrm{i}} \wedge \mathrm{d} p^{\mathrm{j}} \\
& =\frac{1}{2}\left(\zeta_{\alpha}^{\mu} \zeta_{\beta}^{\nu}-\zeta_{\beta}^{\mu} \zeta_{\alpha}^{\nu}\right) \frac{\partial \pi^{\alpha}}{\partial p^{\mathrm{i}}} \frac{\partial \pi^{\beta}}{\partial p^{\mathrm{j}}} \mathrm{d} p^{\mathrm{i}} \wedge \mathrm{d} p^{\mathrm{j}} .
\end{aligned}
$$

Its squared norm is

$$
\left|A^{\prime \mu \nu}\right|^{2}=\frac{1}{4} t^{\mathrm{i}} t^{\mathrm{jl}}\left(\zeta_{\alpha}^{\mu} \zeta_{\beta}^{\nu}-\zeta_{\beta}^{\mu} \zeta_{\alpha}^{\nu}\right)\left(\zeta_{\gamma}^{\mu} \zeta_{\delta}^{\nu}-\zeta_{\delta}^{\mu} \zeta_{\gamma}^{\nu}\right) \frac{\partial \pi^{\alpha}}{\partial p^{\mathrm{i}}} \frac{\partial \pi^{\beta}}{\partial p^{\mathrm{j}}} \frac{\partial \pi^{\gamma}}{\partial p^{\mathrm{k}}} \frac{\partial \pi^{\delta}}{\partial p^{1}}
$$

and expressing $t^{\mathrm{ij}}=e_{\mathrm{m}}^{\mathrm{i}} e_{\mathrm{n}}^{\mathrm{j}} \delta^{m n}$, summing over $\mu$ and $\nu$ and using the definition of the deformation matrix $J_{\mathrm{m}}^{\mu}=e_{\mathrm{m}}^{\mathrm{i}}\left(\partial \pi^{\alpha} / \partial p^{\mathrm{i}}\right) \zeta_{\alpha}^{\mu}$ (see equation (126) gives

$$
\begin{aligned}
\sum_{\mu, \nu}\left|A^{\prime \mu \nu}\right|^{2} & =\left(\operatorname{tr}\left[J J^{\mathrm{T}}\right]\right)^{2}-\operatorname{tr}\left[J J^{\mathrm{T}}\right]^{2} \\
& =(\operatorname{tr}[D])^{2}-\operatorname{tr}[D]^{2} \\
& =\lambda_{1}^{2} \lambda_{2}^{2}+\lambda_{2}^{2} \lambda_{3}^{2}+\lambda_{3}^{2} \lambda_{1}^{2}
\end{aligned}
$$

This expression is completely general, allowing for any spatial and target manifold. Specializing again to the case of $S^{3}$ and $\mathrm{SU}(2)$ we obtain

$$
A^{\mu \nu}=\epsilon^{\mu \nu \sigma} A_{\sigma}
$$

where

$$
A_{\sigma}=\epsilon_{\sigma \mu \nu} \mathcal{L}_{\alpha}^{\mu} \mathcal{L}_{\beta}^{\nu} \mathrm{d} \pi^{\alpha} \wedge \mathrm{d} \pi^{\beta}
$$


Then

$$
A_{\sigma}^{\prime}=\epsilon_{\sigma \mu \nu} \mathcal{L}_{\alpha}^{\mu} \mathcal{L}_{\beta}^{\nu} \frac{\partial \pi^{\alpha}}{\partial p^{\mathrm{i}}} \frac{\partial \pi^{\beta}}{\partial p^{\mathrm{j}}} \mathrm{d} p^{\mathrm{i}} \wedge \mathrm{d} p^{\mathrm{j}}
$$

and

$$
\begin{aligned}
\left|A^{\prime \mu \nu}\right|^{2} & =\epsilon_{\sigma \mu \nu} \epsilon_{\sigma \lambda \rho} t^{\mathrm{ik}} t^{\mathrm{j} \mathrm{l}} \mathcal{L}_{\alpha}^{\mu} \mathcal{L}_{\beta}^{\nu} \mathcal{L}_{\gamma}^{\lambda} \mathcal{L}_{\delta}^{\rho} \frac{\partial \pi^{\alpha}}{\partial p^{\mathrm{i}}} \frac{\partial \pi^{\beta}}{\partial p^{\mathrm{j}}} \frac{\partial \pi^{\gamma}}{\partial p^{\mathrm{k}}} \frac{\partial \pi^{\delta}}{\partial p^{\mathrm{l}}} \\
& =R^{\prime}
\end{aligned}
$$

by $(137)$ and (138). We easily verify that

$$
R_{\mathrm{ijkl}}^{\prime}=A_{\mathrm{ij}}^{\prime \mu \nu} A_{\mathrm{kl}}^{\sigma \tau \tau} \delta_{\mu \sigma} \delta_{\nu \tau}
$$

This expression for $R^{\prime}$ and the Skyrme term is in fact identical to that given in Manton 28], however there is a slight formal difference. We have pulled-back the area elements from the target space $(\mathrm{SU}(2))$ to the spatial manifold $S^{3}$ and computed the sum of their squared norms there. Manton 28 takes the area elements in the spatial manifold and pushes forward their dual area elements (tangent space tensors) to the target space and computes their squared norm in the target space. This gives the same energy functional.

To complete our treatment of this example of $S^{3}$ mapped to $\mathrm{SU}(2)$, we show the interpretation of the third invariant. Consider the integral coming from the third invariant (45)

$$
\begin{aligned}
\int_{\mathrm{S}} \sqrt{I_{3}} \sqrt{\operatorname{det} t} \mathrm{~d}^{3} p & =\int_{\mathrm{S}} \sqrt{\operatorname{det} D} \sqrt{\operatorname{det} t} \mathrm{~d}^{3} p \\
& =\int_{\mathrm{S}} \lambda_{1} \lambda_{2} \lambda_{3} \sqrt{\operatorname{det} t} \mathrm{~d}^{3} p \\
& =\int_{\mathrm{S}} \operatorname{det} J \sqrt{\operatorname{det} t} \mathrm{~d}^{3} p \\
& =\int_{\mathrm{S}} \operatorname{det}\left(e_{\mathrm{m}}^{\mathrm{i}} \frac{\partial \pi^{\alpha}}{\partial p^{\mathrm{i}}} \zeta_{\alpha}^{\mu}\right) \sqrt{\operatorname{det} t} \mathrm{~d}^{3} p \\
& =\int_{\mathrm{S}} \operatorname{det}\left(\frac{\partial \pi}{\partial p}\right) \operatorname{det} \zeta \mathrm{d}^{3} p \\
& =(\operatorname{deg} \pi) \int_{\Sigma} \sqrt{\operatorname{det} \tau} \mathrm{d}^{3} \pi
\end{aligned}
$$

where the factor $\operatorname{deg} \pi$ counts the number of times that the mapping $\pi$ wraps the initial manifold over the target manifold. In reality the last equation is only 
valid locally on $S$, the integral gives the volume of the region covered in $\Sigma$. This volume must be counted with the appropriate sign depending on whether the relative orientation is preserved. Between regions where the relative orientation changes sign is a zero of at least one of the $\lambda$ 's. This gives rise to natural boundaries which should be considered since the sign does not change within these regions. Then summing up the volumes of the regions of $\Sigma$ with the corresponding sign gives exactly the degree of the mapping $\pi$, i.e. the number of complete covering of $\Sigma$ that the mapping $\pi$ provides, multiplied by the volume of $\Sigma$. We assume that $S$ is a manifold without a boundary hence the mapping $\pi$ must cover $\Sigma$ an integral number of times.

From the previous equation, we have the integral

$$
\operatorname{deg} \pi=\frac{1}{\operatorname{Vol} \Sigma} \int_{\mathrm{S}} \operatorname{det}\left(\frac{\partial \pi}{\partial p}\right) \operatorname{det} \zeta \mathrm{d}^{3} p
$$

which can be expressed in terms of $U(p)$ to re-obtain the usual form of the baryon number (9)

$$
\begin{aligned}
\operatorname{deg} \pi & =\frac{1}{2 \pi^{2}} \int_{\mathrm{S}} \operatorname{det}(\mathcal{L}) \operatorname{det}\left(\frac{\partial \pi}{\partial p}\right) \mathrm{d}^{3} p \\
& =\frac{1}{2 \pi^{2}} \int_{\mathrm{S}} \operatorname{det}\left(\mathcal{L} \frac{\partial \pi}{\partial p}\right) \mathrm{d}^{3} p \\
& =\frac{1}{2 \pi^{2}}\left(\frac{-\mathrm{i}}{2}\right)^{3} \int_{\mathrm{S}} \operatorname{det}\left(\operatorname{tr}\left[\tau^{\mu} U^{\dagger} \frac{\partial U}{\partial \pi^{\alpha}}\right] \frac{\partial \pi^{\alpha}}{\partial p^{\mathrm{i}}}\right) \mathrm{d}^{3} p \\
& =\frac{\mathrm{i}}{16 \pi^{2}} \int_{\mathrm{S}} \operatorname{det}\left(\operatorname{tr}\left[\tau^{\mu} U^{\dagger} \frac{\partial U}{\partial p^{\mathrm{i}}}\right]\right) \mathrm{d}^{3} p \\
& =\frac{\mathrm{i}}{16 \pi^{2}} \int_{\mathrm{S}} \epsilon_{\mu \nu \lambda} \operatorname{tr}\left[\tau^{\mu} U^{\dagger} \frac{\partial U}{\partial p^{\mathrm{i}}}\right] \operatorname{tr}\left[\tau^{\nu} U^{\dagger} \frac{\partial U}{\partial p^{\mathrm{j}}}\right] \operatorname{tr}\left[\tau^{\lambda} U^{\dagger} \frac{\partial U}{\partial p^{\mathrm{k}}}\right] \epsilon^{\mathrm{ijk}} \mathrm{d}^{3} p \\
& =\frac{1}{24 \pi^{2}} \int_{\mathrm{S}} \operatorname{tr}\left[U^{\dagger} \partial_{\mathrm{i}} U^{\dagger} U_{\mathrm{j}} U U^{\dagger} \partial_{\mathrm{k}} U\right] \epsilon^{\mathrm{ijk}} \mathrm{d}^{3} p
\end{aligned}
$$

which is the familiar form of the baryon number in the Skyrme model.

The Skyrme energy function, according to Manton's formalism[28], can be expressed as

$$
E=\int_{\mathrm{S}} \sqrt{\operatorname{det} t} \mathrm{~d}^{3} p\left(\lambda_{1}^{2}+\lambda_{2}^{2}+\lambda_{3}^{2}+\lambda_{1}^{2} \lambda_{2}^{2}+\lambda_{2}^{2} \lambda_{3}^{2}+\lambda_{3}^{2} \lambda_{1}^{2}\right)
$$

which is easily re-expressed as 


$$
\begin{gathered}
E=\int_{\mathrm{S}} \sqrt{\operatorname{det} t} \mathrm{~d}^{3} p\left[\left(\lambda_{1} \pm \lambda_{2} \lambda_{3}\right)^{2}+\left(\lambda_{2} \pm \lambda_{3} \lambda_{1}\right)^{2}+\left(\lambda_{3} \pm \lambda_{1} \lambda_{2}\right)^{2}\right] \\
\mp 6 \int_{\mathrm{S}} \sqrt{\operatorname{det} t} \mathrm{~d}^{3} p \lambda_{1} \lambda_{2} \lambda_{3} .
\end{gathered}
$$

This shows a novel way of demonstrating the Bogomolnyi bound: evidently

$$
E \geq 6\left|\int_{\mathrm{S}} \lambda_{1} \lambda_{2} \lambda_{3} \sqrt{\operatorname{det} t} \mathrm{~d}^{3} p\right| .
$$

The equality is attained only if (for winding number +1 )

$$
\lambda_{1}=\lambda_{2} \lambda_{3} \quad \lambda_{2}=\lambda_{3} \lambda_{1} \quad \lambda_{3}=\lambda_{1} \lambda_{2} .
$$

This system has only three distinct solutions:

$$
\left(\lambda_{1}, \lambda_{2}, \lambda_{3}\right):(0,0,0), \quad(1,1,1), \quad(1,-1,-1)+2 \text { permutations. }
$$

The trivial solution corresponds to mapping $S$ to a single point in $\Sigma$ and is the usual vacuum solution. The third set of solutions is equivalent to the second solution after a rotation by $180^{\circ}$ in the cotangent space of the target manifold about a fixed axis. The second solution implies that the map is everywhere an isometry, i.e. , the two $S^{3}$ have the same radius, 1 . This shows that for an infinite initial sphere, which corresponds to the case of $\mathbb{R}^{3}$, the Bogomolnyi bound is not saturated and, as is well known, the map is far from the identity map. Manton and Ruback 27 and Manton 28 show that as the radius of the initial 3-sphere decreases, the map attains the form of the identity for a radius of $\sqrt{2}$. For more details and further applications we refer the reader to the literature[27,28,29,45,46].

We close this section with a few general words on this formalism. First of all the $\lambda$ 's are not independent dynamical variables. Infinitesimal arbitrary perturbations are allowed, however integrating to finite deformations is subject to consistency conditions. For example there is no deformation of a given configuration which can yield $\lambda_{\mathrm{i}}=1$ over a finite region, if the region is not iso-metric to a part of the target manifold. Actually a smooth mapping $\pi$ will always give rise to a smooth set of $\lambda$ 's. A configuration with a discontinuous set of $\lambda$ 's is not attainable even though the corresponding energy integral is finite.

We have also made the intuitive paradigm that the Jacobian matrix is a measure of the deformation, and hence of the energy. However this is somewhat misleading since $\lambda_{i}=0$ is clearly a very deformed situation all the same corresponding to zero energy density. For a physical elastic body, $\lambda_{\mathrm{i}}=0$ is in 
fact an infinite-energy deformation hence the corresponding energy functional is not at all like the Skyrme energy functional. Very schematically, the energy density of an elastic body is

$$
\epsilon_{\text {el.body }} \sim(D-I)^{2}
$$

while in the Skyrme model it is like

$$
\epsilon_{\mathrm{Sk} .} \sim D+D^{2}
$$

Thus the Skyrme ground state is around $D=0$ which is quite unlike the case of the elastic body, where $D=1$.

This completes our exposition of the interpretation of a field theory, specifically the Skyrme model, as a non-linear elasticity theory. In the next two subsections we will look in more detail at the Skyrme model and its static, low energy configurations. First we will elaborate on the instanton method for obtaining an analytical ansatz for the set of relevant low energy configurations, and second we will describe the use of rational maps to obtain reasonable ansätze for multi-baryonic minima.

\subsection{The instanton method}

The instanton method uses the known solutions of 4-dimensional Euclidian Yang-Mills theory called instantons 47 and their moduli spaces to obtain Skyrme field configurations. The relation between the two seems tenuous at first, however, the known global topology and symmetries of the instanton moduli space and its similarities to expected properties of low-energy Skyrme field configurations seems to point in that direction. Consider the case of $B=2$, here know for two widely separated Skyrmions, there are 12 independent degrees of freedom. We expect the relevant low-energy space of configurations to also have 12 dimensions. Manton 37] proposed that this sub-manifold could be obtained as the union of all gradient flow curves linking together all the low-energy critical points. We will return to this subject in much detail in section 4.

This idea to obtain the 12 dimensional sub-manifold, $\mathcal{M}_{12}$ which should serve as the correct truncation of the full field theory description of the interactions and dynamics of two Skyrmions, from gradient flow curves although in principle sound is in practice only numerically, approximately implementable. A consideration of the symmetries involved, led Atiyah and Manton 30 to suggest that an analytical construction of a manifold, which might be a reasonable 
approximation to the true sub-manifold, could be obtained from certain instanton configurations and their holonomies.

The observation consists of the following two steps. First of all, from any SU(2) instanton configuration in $\mathbb{R}^{4}$, it is possible to obtain a unitary matrix valued field defined on $\mathbb{R}^{3}$ by

$$
U(\vec{x})=P \exp \left\{-\int_{-\infty}^{+\infty} \mathrm{d} \tau A_{0}(\vec{x}, \tau)\right\}
$$

where $P$ denotes the path ordered integral. We will show later, how the baryon number of $U(\vec{x})$ is equal to the instanton number of $A_{\mu}(\vec{x}, \tau)$. Secondly it has been known that the space of configurations of two instantons interpolates continuously and smoothly from an axially symmetric, localized configuration to two, individual, "spherically" symmetric, well (infinitely) separated instanton configurations. It is a matter of verification that the corresponding Skyrmion holonomies interpolate smoothly between the toroidal lowest energy deuteron to two infinitely separated single Skyrmions. It is also possible to obtain configurations which correspond to the spherically symmetric dibaryon type configuration.

The most vexxing problem is that the two instanton configurations are 16 dimensional, that is they have 16 independent parameters. One of these corresponds to a global time translation, the integral over the time direction removes this degree of freedom, leaving 15. This is larger than the 12 dimensional manifold which is being sought. It is not evident what is the proper way to reduce the number of parameters by three. Nominally one should reimplement the gradient flow method on this sub-manifold of the configuration space starting from the highest energy critical point of the Skyrme energy functional restricted to the sub-manifold. Such a calculation has not been effected, numerically it is just as difficult to work with a discretized version of the full problem rather than the one defined on the sub-manifold, hence there does not seem to be a compelling motivation to study the gradient flow here. The problem has been studied in detail for the case of the most attractive channel, which we will return to a little later. The instanton method for three and higher baryons is not very efficient.

\subsubsection{Topological numbers}

First let us solidify the connection between baryon number and instanton number. An instanton configuration with instanton number $k$ is technically defined as a connection on an $\mathrm{SU}(2)$ principal bundle over the four-sphere $S^{4}$ with second Chern number $C_{2}=k[26$. The relationship between a gauge field defined 
on $\mathbb{R}^{4}$ and $S^{4}$ is obtained via stereographic projection. The usual instanton configurations which satisfy the Yang-Mills equations of motion defined on $\mathbb{R}^{4}$ have non zero field strength $F_{\mu \nu}$ in a localized region of space-time, and achieve a pure gauge field type configuration towards Euclidian infinity. This means that on the manifold at infinity of $\mathbb{R}^{4}$, which is topologically $S^{3}$, an instanton configuration defines a smooth group element valued configuration $\left.U(\vec{x}, \tau)\right|_{(\vec{x}, \tau) \rightarrow \infty}$ and the gauge field is given by

$$
A_{\mu}=U \partial_{\mu} U^{\dagger}
$$

The second Chern number corresponds to the integral

$$
\begin{aligned}
k & =\frac{-1}{32 \pi^{2}} \int \mathrm{d}^{4} x \epsilon^{\mu \nu \lambda \tau} \operatorname{tr}\left(F_{\mu \nu} F_{\lambda \tau}\right) \\
& =\frac{1}{32 \pi^{2}} \int \mathrm{d}^{4} x \partial_{\mu} K^{\mu} \\
& =\frac{1}{32 \pi^{2}} \oint_{\infty} \mathrm{d} \sigma_{\mu} K^{\mu}
\end{aligned}
$$

where

$$
K^{\mu}=-2 \epsilon^{\mu \nu \lambda \sigma} \operatorname{tr}\left(A_{\nu} F_{\lambda \sigma}-\frac{2}{3} A_{\nu} A_{\lambda} A_{\sigma}\right)
$$

and

$$
F_{\mu \nu}=\partial_{\mu} A_{\nu}-\partial_{\nu} A_{\mu}+\left[A_{\mu}, A_{\nu}\right]
$$

Replacing $A_{\mu}=U \partial_{\mu} U^{\dagger}$ gives

$$
K^{\mu}=\frac{4}{3} \epsilon^{\mu \nu \lambda \sigma} \operatorname{tr}\left(U \partial_{\nu} U^{\dagger} U \partial_{\lambda} U^{\dagger} U \partial_{\sigma} U^{\dagger}\right)
$$

and $k$ is given by the integral

$$
k=W(U)=\frac{1}{24 \pi^{2}} \oint_{\infty} \mathrm{d} \sigma_{\mu} \epsilon^{\mu \nu \lambda \sigma} \operatorname{tr}\left(U \partial_{\nu} U^{\dagger} U \partial_{\lambda} U^{\dagger} U \partial_{\sigma} U^{\dagger}\right) .
$$

This is exactly the measure of the winding number of the mapping of $S^{3} \rightarrow$ $\mathrm{SU}(2)$ defined by the group element at infinity. These mappings define elements of the homotopy group

$$
\Pi_{3}(\mathrm{SU}(2))=\mathbb{Z}
$$


the integer corresponding to the Chern number. The configuration on $\mathbb{R}^{4}$ does not attain a constant value at infinity, hence it is difficult to interpret this field as corresponding to a field on $S^{4}$ after stereographic projection. (To be precise, the stereographic projection involved is actually the conformal mapping taking $S^{4} \rightarrow \mathbb{R}^{4} \cdot g_{\mu \nu}=1 /\left(|x|^{2} / 4 R^{2}+1\right)^{2} \eta_{\mu \nu}$ is the conformal transformation taking $\mathbb{R}^{4}$ to $S^{4}$ with the coordinate transformation exactly as in equation (106). This mapping has the advantage of mapping solutions of the equations of motion on one manifold to solutions on the other because the Lagrangian of YangMills theories is conformally invariant 48.). However the configuration on $S^{4}$ is also somewhat subtle, $A_{\mu}$ is actually a connection on a non-trivial $\mathrm{SU}(2)$ principal bundle defined over the base manifold $S^{4}$. These bundles are specified by fixing the transition function which maps the fibre $\mathrm{SU}(2) \equiv S^{3}$ over the "northern hemisphere" of $S^{4}$ to the $S^{3}$ over the "southern hemisphere" at the "equator". The equator of $S^{4}$ is simply an $S^{3}$. Hence the transition functions are tantamount to defining a group element over the equatorial $S^{3}$. This means that one defines a mapping of the equatorial $S^{3}$ to the group $\mathrm{SU}(2) \equiv S^{3}$. Such mappings fall into the disjoint homotopy classes labelled exactly as in (169). The second Chern number of the bundle (164) is exactly equal to the integer characterizing the homotopy class of the transition function.

Geometrically there is no constraint on the size of the coordinate charts; one need not restrict oneself to equal hemispheres. There is no hindrance to extending the southern hemisphere to include the whole of $S^{4}$ except for one point, the north pole. Indeed, in this way we will extend the solution of the equations of motion to almost everywhere on $S^{4}$. The solution will be singular at the north pole however only for the connection. The integral (164) will be an integral over the whole sphere of only the field strengths associated with the connection and the density $\operatorname{tr}\left(F_{\mu \nu} \tilde{F}^{\mu \nu}\right)$ is non-singular over the whole sphere. The result must still give the second Chern number. Now the conformal projection of this field configuration to $\mathbb{R}^{4}$ will give the field configuration that satisfies the equations of motion on $\mathbb{R}^{4}$ and furthermore whose integral corresponding to (164) is also $C_{2}\left(A_{\mu}\right)$ (since (164) is independent of the metric). The values achieved at the north pole by $A_{\mu}$ defined by the limiting value of the configuration along any path leading to the north pole are all equal modulo gauge transformations. The conformal transformation maps this field on $S^{4}$ to a configuration on $\mathbb{R}^{4}$ which becomes a pure gauge configuration at infinity since the field strength at the north pole is diluted over the entire $S^{3}$ manifold at infinity.

The path ordered integral (162) which defines $U(\vec{x})$ starts at $\tau=-\infty$ and follows a straight line to $\tau=+\infty$. This corresponds to a curve on $S^{4}$ which starts at the north pole, follows a particular path on the $S^{4}$ and returns back to the north pole (see figure (1). The path on $S^{4}$ is simply given by the intersection of the $S^{4}$ with a 2-plane, that which is defined by the line of integration in $\mathbb{R}^{4}$ and the north pole. This intersection is actually just a circle. The set of such 


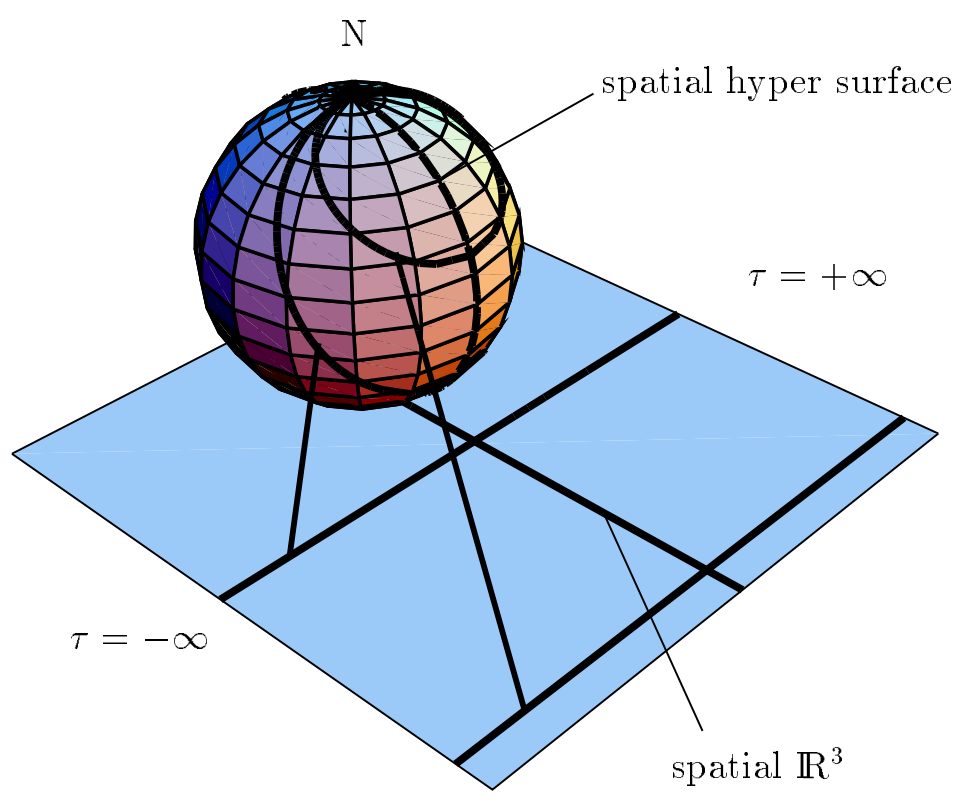

Fig. 4. Diagram of the stereographic projection from $S^{4}$ to $\mathbb{R}^{4}$.

curves on $S^{4}$ leave the north pole on one side of a 3-dimensional hyper surface, circle around the $S^{4}$, and return to the north pole from the other side of the hypersurface. The hypersurface is in fact just a "great" 3-sphere, exactly like the equatorial $S^{3}$ except that it passes from north pole to south pole, and back. It is exactly the inverse (stereographic) projection of the spatial $\mathbb{R}^{3}$ (of the $\mathbb{R}^{4}$ ) onto the $S^{4}$. The curves leave the north pole, intersect this great $S^{3}$ exactly once and come back to the north pole in a symmetric fashion. Hence each curve on the $S^{4}$ defines a unitary matrix valued configuration on the great $S^{3}$ at the point where the curve intersects this great $S^{3}$. This configuration is by construction continuous. Consequently we manage to define a winding number (169).

The winding number is invariant under any continuous deformation which keeps a one to one relation between each curve and the points of the hypersurface. Envisage the following deformations. The lines of integration are well represented by the lines of forces emanating from and returning to an ideal pointlike "dipole" situated at the north pole. The great sphere separates these lines of force at the north pole into outgoing lines on one side, and incoming lines on the other side. We simply imagine moving the two charges comprising the dipole apart, keeping the lines of integration the same as the lines of forces 
leaving the positive charge on one side and arriving at the negative charge on the other. Such a modification of the lines of integration will result in a homotopy of the original $U(\vec{x})$, and hence will not change the homotopy type. Finally we will arrive at the situation where the two charges occupy antipodal points of the four sphere (actually on the equator) and the lines of force emanate symmetrically from one charge, cross the great 3-sphere and finally terminate on the opposite charge at the antipodal point. We make one further homotopy, we rigidly rotate the system of charges, lines of integration and the great $S^{3}$ until they are vertical, such that the positive charge is at the south pole and the negative charge is at the north pole, and the usual equator now corresponds to the great $S^{3}$. Such a deformation requires a simultaneous redefinition of the stereographic projection, and a deformation of the $U(\vec{x})$ (because the integration lines are changing) but it is clearly a continuous deformation keeping the homotopy type invariant. Finally to be complete, we had started with closed line integrals originally (leaving and returning to the north pole) but now we have open line integrals starting at the south pole and terminating at the north pole. We can easily remedy this by adding one path to all of the others, starting at the north pole and descending down a fixed meridian to the south pole for all of the line integrals. This simply left-multiplies each $U(\vec{x})$ by a constant unitary matrix, which again does not modify the homotopy type. Closed contour path ordered exponential integrals are gauge covariant hence we can return to the original description of the instanton on $S^{4}$ with more than two patches. Now we have arrived at the starting point of the demonstration given in Manton and Atiyah[31] where they show that the winding number of this configuration is the same as the instanton number (the second Chern number of the instanton bundle in question). This demonstration proceeds as follows.

The first step is to use the gauge freedom to put the gauge field in the specific gauge where the component of the gauge field along the meridional directions vanishes. This can be established in each patch separately. Then the integral

$$
P \exp \left(-\int A_{\mu} d x^{\mu}\right)=1
$$

since the inner product $A_{\mu} d x^{\mu}$ is zero along the meridional path. However the definition of the path ordered exponential, when the path of integration crosses a boundary between patches is such that one must multiply the contribution coming from the first leg of the path by the transition function before continuing with the integral in the second patch (see figure 5).

$$
P \exp \left(-\int_{0}^{1} A_{\mu} d x^{\mu}\right)=P \exp \left(-\int_{\tau_{0}}^{1} A_{\mu} d x^{\mu}\right) G(x) P \exp \left(-\int_{0}^{\tau_{0}} A_{\mu} d x^{\mu}\right) .
$$




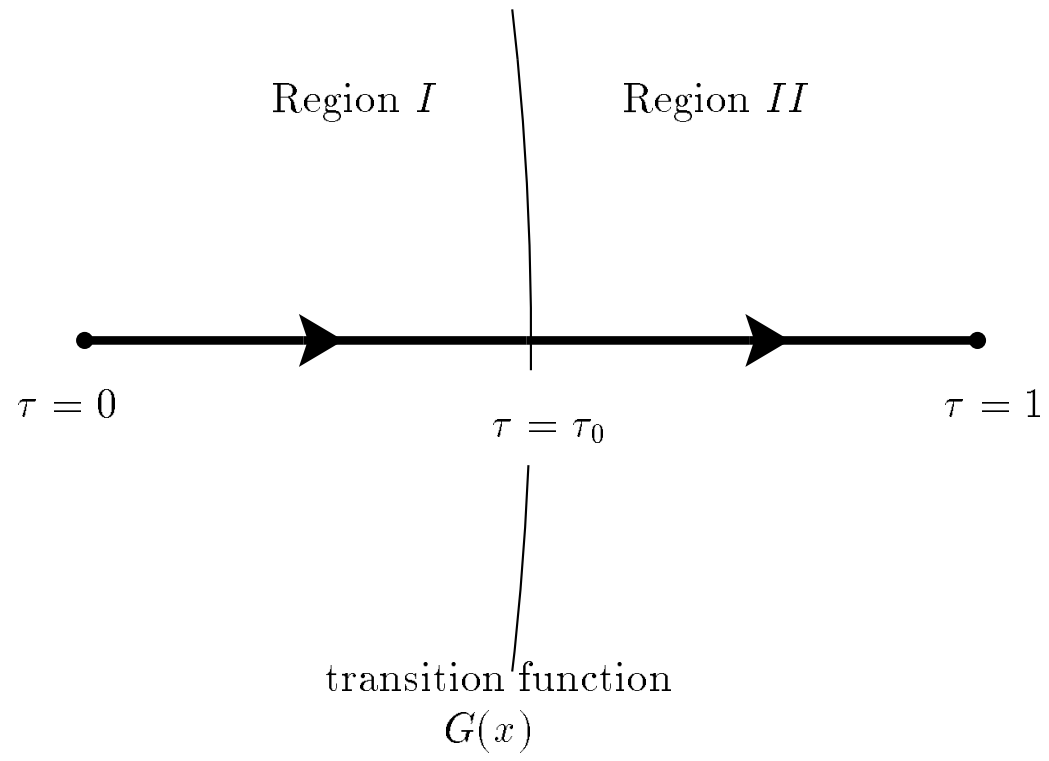

Fig. 5. Diagram of the role of the transition function $G(x)$ between regions $I$ and $I I$.

Such an expansion is gauge covariant under simultaneous independent gauge transformations in the patches $I$ and $I I$ since the transition function is defined to transform as

$$
G(x) \rightarrow U_{\mathrm{II}} G(x) U_{\mathrm{I}}^{\dagger}
$$

while

$$
\begin{aligned}
& P \exp \left(-\int_{0}^{\tau_{0}} A_{\mu} d x^{\mu}\right) \rightarrow U_{\mathrm{I}}\left(\tau_{0}\right) P \exp \left(-\int_{0}^{\tau_{0}} A_{\mu} d x^{\mu}\right) U_{\mathrm{I}}^{\dagger}(0) \\
& P \exp \left(-\int_{\tau_{0}}^{1} A_{\mu} d x^{\mu}\right) \rightarrow U_{\mathrm{II}}(1) P \exp \left(-\int_{\tau_{0}}^{1} A_{\mu} d x^{\mu}\right) U_{\mathrm{II}}^{\dagger}\left(\tau_{0}\right)
\end{aligned}
$$

yielding

$$
P \exp \left(-\int_{0}^{1} A_{\mu} d x^{\mu}\right) \rightarrow U_{\mathrm{II}}(1) P \exp \left(-\int_{0}^{1} A_{\mu} d x^{\mu}\right) U_{\mathrm{I}}^{\dagger}(0)
$$


Now since $A_{\mu} d x^{\mu}=0$ in our gauge, we obtain

$$
U(\vec{x})=U_{0} G(\vec{x})
$$

where $G(\vec{x})$ is the transition function at the equator. Then, the baryon number

$$
B=W(U(\vec{x}))=W(G(\vec{x}))=C_{2}\left(A_{\mu}\right)=k ;
$$

hence the baryon number and the instanton number are identical.

\subsubsection{The sector $B=1$}

For $k=1$ the instanton profile is given by

$$
A_{\mu}=\mathrm{i} \bar{\sigma}_{\mu \nu} \partial_{\nu} \ln \rho
$$

where the definition of $\bar{\sigma}$ can be found in the article of Jackiw, Nohl and Rebbi (JNR) [48], however the time component is explicitly

$$
A_{4}=\frac{\mathrm{i}}{2} \frac{\nabla \rho}{\rho} \cdot \vec{\tau}
$$

with

$$
\rho=1+\frac{\lambda}{\left(x_{\mu}-X_{\mu}\right)\left(x^{\mu}-X^{\mu}\right)}=1+\frac{\lambda}{|x-X|^{2}} .
$$

This is in the 't Hooft gauge 49. The singularities in $\rho$ are gauge artefacts and hence do not contribute to gauge covariant quantities such as the definition of the Skyrme field. A local gauge transformation moves the singularity to wherever we want, but of course does not affect the $U(\vec{x})$. We will not show this here. The function $\rho$ (180) has 5 parameters, but three others are to be added in because of global gauge transformations, which were factored out in the definition of instantons. This yields 8 parameters. Evidently the 4 translation parameters $X^{\mu}$ are the center of mass coordinates, the spatial ones determine the spatial center of mass of the corresponding Skyrmion, the temporal one is absorbed by the integration in the time direction. Factoring out these four leaves 4 parameters, $\lambda$ governing the overall scale, and three corresponding to global gauge transformations. Hence

$$
\rho=1+\frac{\lambda}{\left(r^{2}+\tau^{2}\right)}
$$


where $r^{2}=\vec{x} \cdot \vec{x}$. Then

$$
A_{4}=-\frac{i}{2} \frac{\lambda}{\rho} \frac{2 \vec{x} \cdot \vec{\tau}}{\left(r^{2}+\tau^{2}\right)^{2}}
$$

and

$$
\begin{aligned}
U(\vec{x}) & =P \exp \left(-\int_{-\infty}^{\infty}-\frac{i}{2} \frac{\lambda}{\rho} \frac{2 \vec{x} \cdot \vec{\tau}}{\left(r^{2}+\tau^{2}\right)^{2}} d \tau\right) \\
& =\exp \left(i \lambda \vec{x} \cdot \vec{\tau} \int_{-\infty}^{\infty} \frac{d \tau}{\left(r^{2}+\tau^{2}\right)^{2}+\lambda\left(r^{2}+\tau^{2}\right)}\right) \\
& =e^{i \hat{x} \cdot \vec{\tau} \pi\left(1-\left(1+\frac{\lambda}{r^{2}}\right)^{-\frac{1}{2}}\right)}
\end{aligned}
$$

Thus

$$
f(r)=\pi\left(1-\left(1+\frac{\lambda}{r^{2}}\right)^{-\frac{1}{2}}\right)
$$

which satisfies $f(0)=\pi$. This point is actually determined by the limit from non-zero values of $r$, since the integral above is not well defined for $r=0$. The singularity is gauge dependent, as mentioned above, hence by a local gauge transformation we can move the singularity away from $r=0$ without affecting the value for the Skyrme field. The Skyrme field so obtained will be continuous at $r=0$, hence the value at $r=0$ can equally well be defined as the value obtained from the limit of non-zero $r$. Furthermore, $f(\infty)=0$. For minimum energy one finds $\lambda=2.109$, and the corresponding energy is $E=1.2432 \times 12 \pi^{2}$. This exceeds the numerically obtained minimum energy solution by only $1 \%$.

Adding in gauge transformations, i.e. global iso-rotations simply combs the Skyrmion profile without affecting the energy. The iso-rotation parameters are already evident in the JNR 48] parametrization of the instantons. Here we have

$$
\rho=\frac{\lambda_{1}}{\left|x-X_{1}\right|^{2}}+\frac{\lambda_{2}}{\left|x-X_{2}\right|^{2}}
$$

which has 10 parameters. It is known that several of these are local gauge artefacts, indeed, the instanton obtained is gauge equivalent to the 't Hooft instanton (180) with

$$
X=\frac{\lambda_{1} X_{2}+\lambda_{2} X_{1}}{\lambda_{1}+\lambda_{2}}
$$




$$
\lambda=\frac{\lambda_{1} \lambda_{2}}{\left(\lambda_{1}+\lambda_{2}\right)^{2}}\left|X_{1}-X_{2}\right|^{2} .
$$

The JNR instanton generates, however, a Skyrme field differing from that obtained from the 't Hooft instanton by a global gauge transformation given by

$$
U_{0}=\frac{\left(X_{2}-X_{1}\right)^{0}}{\left|X_{2}-X_{1}\right|}+\mathrm{i} \frac{\left(\vec{X}_{2}-\vec{X}_{1}\right)}{\left|X_{2}-X_{1}\right|} \cdot \vec{\tau} .
$$

This completes the case $k=1(B=1)$.

\subsubsection{The sector $B=2$}

In this subsection we present the findings of Atiyah and Manton[31], where they relate the parameters (moduli) of $k=2$ instanton configurations introduced analytically by JNR and geometrically by Hartshorne[50], to the various parameters of the corresponding $B=2$ Skyrme configurations.

For $k=2(B=2)$, the JNR parametrization is

$$
\rho=\frac{\lambda_{1}}{\left|x-X_{1}\right|^{2}}+\frac{\lambda_{2}}{\left|x-X_{2}\right|^{2}}+\frac{\lambda_{3}}{\left|x-X_{3}\right|^{2}}
$$

a 15 parameter solution. It is clear that the overall scale of the $\lambda$ 's is never a parameter, yielding 14 parameters. In addition there is an explicit one parameter family of local gauge transformations included in $\rho$, reducing the number of true parameters to 13 . We will return to this redundancy later. Integrating over Euclidean time to obtain the Skyrme field reduces the number of parameters to 12 . Finally putting in the 3 iso-rotational degrees of freedom, as they are not included in the solution, implies that the corresponding Skyrmion fields will have a total of 15 parameters.

In general, for higher $k$, there is no local gauge transformation in the JNR expression for $\rho$, which thus has $5 k+4$ parameters. Integrating over time removes 1 but adding in three for global iso-rotations yields in general $5 k+6$ parameter Skyrme fields. This is obviously not the full complement of $6 k$ that we expect for $B=k$ Skyrmions. The full instanton moduli space is actually supposed to be $8 k$ dimensional. These include 4 positions, 1 scale and 3 isorotations per instanton, not removing the 3 overall iso-rotation parameters. Integrating to get the Skyrmions removes one parameter, implying an $8 k-1$ dimensional manifold. This manifold would correspond to 3 positions, 3 isorotations and 1 scale per Skyrmion, and $k-1$ relative "time" coordinates. These 


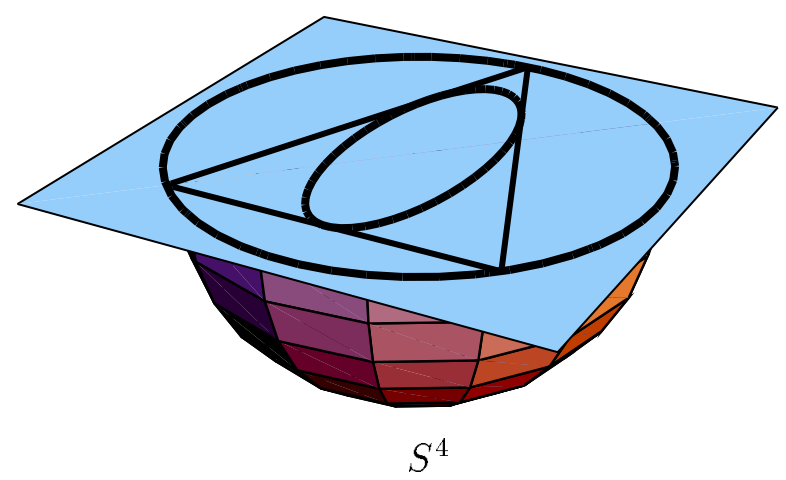

Fig. 6. Diagram of the $S^{4}$ cut by a plane, exhibiting a triangle and ellipse.

time coordinates serve simply to fix the order of the individual Skyrmion fields in the corresponding product ansatz type configuration, if the Skyrmions are well separated in these time coordinates. This ordering is of course quite irrelevant if the Skyrmions are well separated spatially. However the interpolation between different orderings is quite important when they are spatially close together.

The full $8 k$ dimensional manifold of instanton solutions is well understood algebraically but not analytically. The largest manifold of analytically explicit solutions corresponds to the $5 k+4$ dimensional manifold of JNR. For $k=2$ these have 13 parameters and for which there is an algebraic characterization given by Hartshorne [50]. Hartshorne proves that there is a 1-1 correspondence between the instanton solutions (on $S^{4}$ ) and a set of ellipses that are interior to the $S^{4}$ (we imagine that the $S^{4}$ in question is embedded in $\mathbb{R}^{5}$ ). The ellipses lie in a 2-dimensional plane that intersects the $S^{4}$ in a (coplanar) circle, subject to one condition. The condition insists that the ellipse can be circumscribed by a triangle whose vertices lie on the circle (see figure 6). This condition is called the Poncelet condition, and he proved that if such a triangle exists, then there is a one parameter family of such triangles, called a porism 31. The Poncelet condition is effectively one condition on the parameters of the ellipse, for a fixed circle. An ellipse is parametrized in general by 5 parameters: 2 give the semi-major and semi-minor axes, 2 fix the position of the center and one fixes its orientation in the plane relative to a fixed set of coordinate axes (rotations). The Poncelet condition leaves 4 degrees of freedom. Now a 2-plane 
passing through the origin in $\mathbb{R}^{5}$ is specified by a division of the $\mathbb{R}^{5}$ into the 2-dimensional space of the plane and the 3-dimensional space orthogonal to the plane. The action of the orthogonal group $\mathrm{O}(5)$ generates all the different possibilities from any given initial one. However, if the orthogonal group acts only in the 2-plane or in the 3-dimensional orthogonal space, then we obtain nothing new. Hence the dimension of the space of 2-planes in $\mathbb{R}^{5}$ passing through the origin is $\operatorname{dim} \mathrm{O}(5)-(\operatorname{dim} \mathrm{O}(3)+\operatorname{dim} \mathrm{O}(2))=10-(3+1)=6$, where we have used that the dimension of $\mathrm{O}(N)$ is $N(N-1) / 2$. Removing the condition that the plane passes through the origin allows 3 translations, one for each independent orthogonal direction, yielding 9 degrees of freedom. Adding in the four degrees of freedom of the ellipse yields 13 in total.

Given the circle and ellipse, the corresponding family of triangles is neatly described by a cubic equation. Suppose that $s=\tan \left(\frac{\theta}{2}\right)$ is a variable along the circle $(\theta \in[-\pi, \pi]$ an angular variable around the circle). Then the vertices of the triangle correspond to $s_{1}, s_{2}, s_{3}$, which are without loss of generality the roots of a cubic polynomial equation,

$$
p_{0} s^{3}+p_{1} s^{2}+p_{2} s+p_{3}=0 \text {. }
$$

Clearly there are some global constraints that they the $p_{\mathrm{i}}$ 's must satisfy so that the cubic (190) has three real roots, but these constraints do not remove any degrees of freedom. However, the $p_{\mathrm{i}}$ 's are real, their overall scale is irrelevant, and they are not all zero, hence they define a ray in $\mathbb{R}^{4}$. The space of all rays in $\mathbb{R}^{4}$ is called the real projective space of dimension three, denoted $\mathbb{R} P_{3}$. The Poncelet condition is expressed in this light by requiring that the coefficients, of the cubic equations for all the triangles in the porism, must lie along a straight (projective) line in $\mathbb{R} P_{3}$. Hence if

$$
q_{0} s^{3}+q_{1} s^{2}+q_{2} s+q_{3}=0 .
$$

is a cubic whose roots correspond to the vertices of another solution of the Poncelet condition, then all solutions are obtained by the interpolation

$$
\left(\mu p_{0}-\nu q_{0}\right) s^{3}+\left(\mu p_{1}-\nu q_{1}\right) s^{2}+\left(\mu p_{2}-\nu q_{2}\right) s+\left(\mu p_{3}-\nu q_{3}\right)=0 .
$$

Now if for some $\bar{\mu}$ and $\bar{\nu}$ the coefficient $\bar{\mu} p_{0}+\bar{\nu} q_{0}=0$, then the surviving quadratic equation gives two of the roots, while the last root is pushed off to $s= \pm \infty$ (which is the same point for either sign). The requirement that the ellipse be interior to the circle implies that the roots of (192) are real and distinct for all $\mu$ and $\nu$. This last constraint precludes the possibility that also $\bar{\mu} p_{1}+\bar{\nu} q_{1}=0$ since then we get one finite root but two roots get pushed off to $s= \pm \infty$, which means that they are the same point on the circle. This is not permitted for an ellipse that is interior to the circle. 


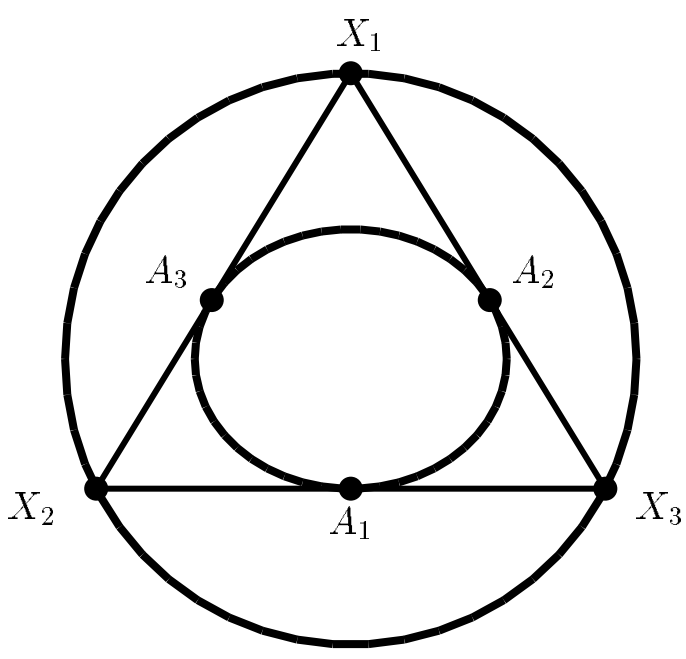

Fig. 7. Diagram of a triangle showing the triangle $X_{1} X_{2} X_{3}$, and the ellipse it defines, touching it at the points $A_{1}, A_{2}$ and $A_{3}$.

The JNR parametrization corresponds to instantons in $\mathbb{R}^{4}$. However these are related to those defined on $S^{4}$ by a conformal transformation. This corresponds to a specific stereographic projection of $S^{4}$ to $\mathbb{R}^{4}$. This projection takes circles to circles and triangles and ellipses in the interior of the $S^{4}$ also to triangles and ellipses, respectively. The JNR parametrization immediately gives us the ellipse and the circle. The points $X_{\mathrm{i}}$ determine a circle and the vertices of a triangle. Then we use

$$
\frac{\lambda_{1}}{\lambda_{2}}=\frac{X_{1} A_{3}}{A_{3} X_{2}}, \quad \frac{\lambda_{2}}{\lambda_{3}}=\frac{X_{2} A_{1}}{A_{1} X_{3}}, \quad \frac{\lambda_{3}}{\lambda_{1}}=\frac{X_{3} A_{2}}{A_{2} X_{1}}
$$

to determine the points $A_{\mathrm{i}}$, which are defined to be on the line joining $X_{\mathrm{j}}$ with $X_{\mathrm{k}}$ (with $i, j, k$ all distinct, and where $X_{1} A_{3}$ signifies the distance between $X_{1}$ and $A_{3}$ along the line joining $X_{1}$ with $X_{2}$ for example). The $\lambda_{\mathrm{i}}$ 's and the $X_{\mathrm{i}}$ 's are part of the JNR parameters, the $A_{\mathrm{i}}$ 's are uniquely determined by the ratios of the $\lambda_{\mathrm{i}}$ 's. The $X_{\mathrm{i}}$ 's determine the circle uniquely, and the ellipse is determined also uniquely by the points $A_{\mathrm{i}}$ along with the condition that the ellipse be circumscribed by the triangle (see figure 7).

The description of the instanton in terms of the triangle and ellipse allows us to readily understand the symmetries of the instanton and hence the resulting Skyrmion. The $\lambda_{\mathrm{i}}$ 's seem to correspond to one triangle in the porism, however they in fact afford an interpretation in terms of an infinitesimal variation of the triangle within the porism. If $X_{\mathrm{i}}^{\prime}$ are the vertices of an infinitesimally close triangle to the one determined by the $X_{\mathrm{i}}$ 's, then the line $X_{1}^{\prime} X_{2}^{\prime}$ is an infinitesimal rotation of the line $X_{1} X_{2}$ about the point of tangency (see figure 8). By elementary geometry 


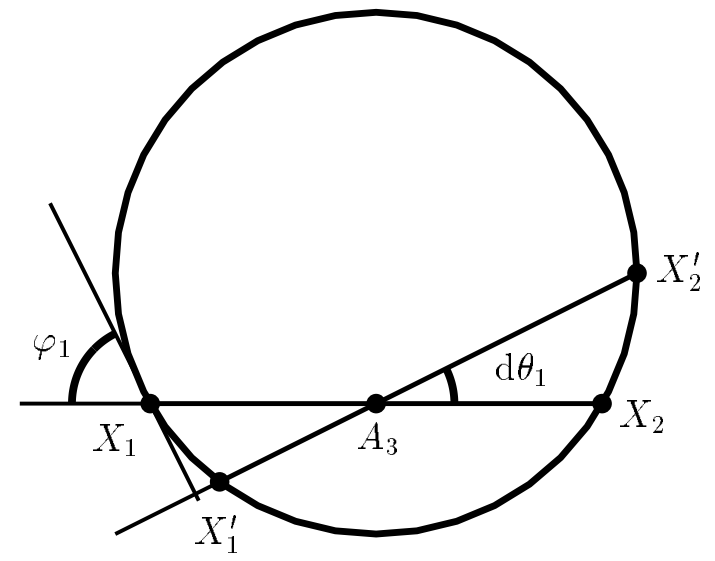

Fig. 8. Diagram of the infinitesimal rotation of $X_{1} X_{2}$ to $X_{1}^{\prime} X_{2}^{\prime}$. Similar rotations occur to the other two sides of the triangle.

$$
\begin{aligned}
& X_{1} X_{1}^{\prime}=\left(X_{1} A_{3}\right) \frac{\mathrm{d} \theta_{1}}{\sin \varphi_{1}} \\
& X_{2} X_{2}^{\prime}=\left(A_{3} X_{2}\right) \frac{\mathrm{d} \theta_{1}}{\sin \varphi_{1}}
\end{aligned}
$$

hence

$$
\frac{X_{1} X_{1}^{\prime}}{X_{2} X_{2}^{\prime}}=\frac{X_{1} A_{3}}{A_{3} X_{2}}=\frac{\lambda_{1}}{\lambda_{2}}
$$

using (193). Thus for an infinitesimal change of the triangle, the vertices move along the circle by a distance proportional to the $\lambda_{\mathrm{i}}$ 's. This means that the JNR data determines a 1 parameter family of cubic equations. With $X_{\mathrm{i}} X_{\mathrm{i}}^{\prime}=$ $\mathrm{d} \theta_{\mathrm{i}} / \sin \varphi_{\mathrm{i}}$, and $s_{\mathrm{i}}=\tan \frac{\theta_{\mathrm{i}}}{2}$, then

$$
\mathrm{d} s_{\mathrm{i}}=\frac{1}{2}\left(1+s_{\mathrm{i}}^{2}\right) \mathrm{d} \theta_{\mathrm{i}}
$$

The family of cubic equations

$$
\begin{aligned}
& \mu\left(s-s_{1}\right)\left(s-s_{2}\right)\left(s-s_{3}\right)+\nu\left(\lambda_{1}\left(1+s_{1}^{2}\right)\left(s-s_{2}\right)\left(s-s_{3}\right)+\right. \\
& \left.\lambda_{2}\left(1+s_{2}^{2}\right)\left(s-s_{3}\right)\left(s-s_{1}\right)+\lambda_{3}\left(1+s_{3}^{2}\right)\left(s-s_{1}\right)\left(s-s_{2}\right)\right)=0
\end{aligned}
$$

has three real roots which correspond to the three angles $\theta_{\mathrm{i}}$. Indeed if $\nu=0$ the roots are $s_{1}, s_{2}$ and $s_{3}$.

For an infinitesimal $\nu, s_{\mathrm{i}}=s_{\mathrm{i}}+d s_{\mathrm{i}}$, solving to first non-trivial order yields 


$$
\begin{aligned}
& \mu d s_{1} d s_{2} d s_{3}+ d \nu\left(\lambda_{1}\left(1+s_{1}^{2}\right) d s_{2} d s_{3}+\right. \\
&\left.\lambda_{2}\left(1+s_{2}^{2}\right) d s_{1} d s_{3}+\lambda_{3}\left(1+s_{3}^{2}\right) d s_{1} d s_{2}\right)=0
\end{aligned}
$$

implying $\mathrm{d} s_{\mathrm{i}}=\frac{-3 \mathrm{~d} \nu}{\mu} \lambda_{\mathrm{i}}\left(1+s_{\mathrm{i}}^{2}\right)$ which corresponds to the desired variation (197).

Now we can address the case where the circle on $S^{4}$ passes through the "north pole", the point from which we do the stereographic projection. In this case the circle projects to a straight line in $\mathbb{R}^{4}$ and the ellipse also projects to the same line. The projections of the porism of triangles gives a triplet of points along the line, coming from the vertices. These triples are again the roots of a cubic equation as before, however the parameter is just an affine parameter along the line. If $X_{\mathrm{i}}$ and $X_{\mathrm{i}}^{\prime}$ are two infinitesimally separated triplets, we can define weights (up to an overall constant) by

$$
\frac{\lambda_{1}}{\lambda_{2}}=\frac{X_{1} X_{1}^{\prime}}{X_{2} X_{2}^{\prime}} \quad \frac{\lambda_{2}}{\lambda_{3}}=\frac{X_{2} X_{2}^{\prime}}{X_{3} X_{3}^{\prime}} \quad \frac{\lambda_{3}}{\lambda_{1}}=\frac{X_{3} X_{3}^{\prime}}{X_{1} X_{1}^{\prime}}
$$

then the JNR potential is as before. Conversely, given the $X_{\mathrm{i}}$ along a line in $\mathbb{R}^{4}$, we may invert the stereographic projection and reconstruct the instanton and its associated circle and ellipse on $S^{4}$ equally well. The inverse images of the $X_{\mathrm{i}}$ 's determine the circle and the vertices of the triangle, the weights determine the ellipse. The corresponding line of cubics is defined directly for the affine parameter $s$ on the line in $\mathbb{R}^{4}$. An infinitesimal variation (200) implies the variation

$$
\mathrm{d} s_{\mathrm{i}}=\lambda_{\mathrm{i}} \mathrm{d} s .
$$

Then the projective line of cubics is

$$
\begin{array}{r}
\mu\left(s-s_{1}\right)\left(s-s_{2}\right)\left(s-s_{3}\right)+\nu\left[\lambda_{1}\left(s-s_{2}\right)\left(s-s_{3}\right)+\right. \\
\left.\lambda_{2}\left(s-s_{3}\right)\left(s-s_{1}\right)+\lambda_{3}\left(s-s_{1}\right)\left(s-s_{2}\right)\right]=0
\end{array}
$$

and an infinitesimal variation $\mathrm{d} \nu$ yields $d s_{\mathrm{i}}=\frac{-3 d \nu}{\mu} \lambda_{\mathrm{i}}$ in concord with (201). The corresponding points on $S^{4}$ projecting to these roots gives the porism of triangles there.

We will next consider two special cases which give rise to interesting $B=2$ Skyrmions. First consider the case of an ellipse of very high eccentricity, such that it almost touches the surface of the sphere $S^{4}$ at two points (see figure 9). The instanton degenerates to two $k=1$ instantons near these points. Stereographic projection gives 2 well separated and localized instantons in $R^{4}$. For 


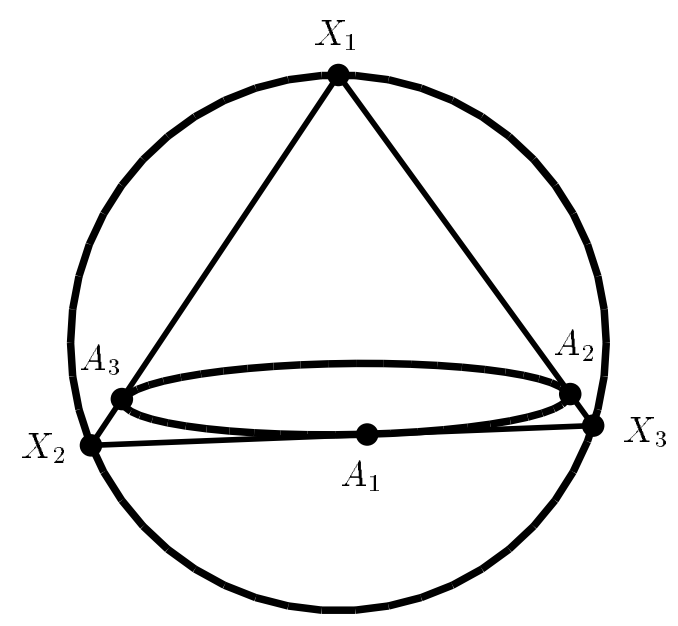

Fig. 9. Triangle corresponding to a pair of well separated instantons or, correpondingly, to two well separated Skyrmions in the product ansatz.

simplicity we take $X_{\mathrm{i}}$ not be collinear. With $\lambda_{1}=1$ (without loss of generality) and $\lambda_{2}, \lambda_{3}<<1$ then for $x$ near $X_{2}$ the JNR potential is approximately

$$
\rho=\frac{1}{\left|x-X_{1}\right|^{2}}+\frac{\lambda_{2}}{\left|x-X_{2}\right|^{2}}+\frac{\lambda_{3}}{\left|x-X_{3}\right|^{2}} \approx \frac{1}{\left|x-X_{1}\right|^{2}}+\frac{\lambda_{2}}{\left|x-X_{2}\right|^{2}}
$$

which is exactly the JNR potential for a single instanton centered at $\frac{X_{2}+\lambda_{2} X_{1}}{\left(1+\lambda_{2}\right)} \approx$ $X_{2}$ with scale parameter $\lambda_{2}\left|X_{2}-X_{1}\right|^{2}$. The scale being proportional to $\lambda_{2}$, this allows for the possibility that the instanton here has little overlap with the corresponding one located at $X_{3}$. The integration along the time lines gives two well localized Skyrmions. The minimum energy single Skyrmions are obtained for $\lambda \approx 2.109$, which is not particularly small. However given that the scale size of the instantons is quadratically dependent on the separation, it is always possible to choose this so that the resulting single Skyrmions are well separated and of minimal energy.

If $X_{1}$ is relatively near $X_{2}$ and it changes while keeping everything else fixed, the Skyrmion at position $X_{2}$ will vary over all possible orientations while the one at $X_{3}$ will remain essentially unchanged. (203) implies a gauge transformation (188)

$$
U=\frac{\left(X_{2}-X_{1}\right)^{0}}{\left|X_{2}-X_{1}\right|}+\mathrm{i} \frac{\left(\vec{X}_{2}-\vec{X}_{1}\right)}{\left|X_{2}-X_{1}\right|} \cdot \vec{\tau} .
$$

which covers $\mathrm{SO}(3)$, hence all possible orientations, twice as $X_{1}$ varies over a 3 sphere centered on $X_{2}$. Hence all possible relative orientations are permitted and this case contains all well separated Skyrmions which gives rise to the product ansatz. 
The second case that we will consider is the spherically symmetric situation, which gives rise to hedgehog fields for $B=2$. Consider the potential

$$
\begin{aligned}
\rho & =\frac{\lambda_{1}}{r^{2}+\left(\tau-\tau_{1}\right)^{2}}+\frac{\lambda_{2}}{r^{2}+\left(\tau-\tau_{2}\right)^{2}}+\frac{\lambda_{3}}{r^{2}+\left(\tau-\tau_{3}\right)^{2}} \\
& =\frac{N(r, \tau)}{\prod_{\mathrm{i}=1}^{3}\left(r^{2}+\left(\tau-\tau_{\mathrm{i}}\right)^{2}\right)}
\end{aligned}
$$

with $r^{2}=\vec{x} \cdot \vec{x}$. The corresponding JNR instantons are situated along the time axis. $N(r, \tau)$ is a quartic polynomial in $\tau$ with $r$ dependent coefficients which is positive since the denominators can never simultaneously vanish. $N$ is actually gauge invariant. $N$ has $r$ dependent roots $\alpha(r), \alpha^{*}(r), \beta(r), \beta^{*}(r)$, with the imaginary parts of $\alpha$ and $\beta, \Im(\alpha), \Im(\beta)>0$. Then

$$
\begin{aligned}
\ln \rho=\ln (\tau-\alpha)+ & \ln \left(\tau-\alpha^{*}\right)+\ln (\tau-\beta)+\ln \left(\tau-\beta^{*}\right) \\
& -\sum_{\mathrm{j}=1}^{3}\left(\ln \left(\tau-\tau_{\mathrm{j}}-\mathrm{i} r\right)+\ln \left(\tau-\tau_{\mathrm{j}}+\mathrm{i} r\right)\right)
\end{aligned}
$$

which in turns yields

$$
\begin{aligned}
\frac{1}{\rho} \frac{\mathrm{d} \rho}{\mathrm{d} r}=-\frac{1}{(\tau-\alpha)} \frac{\mathrm{d} \alpha}{\mathrm{d} r} & -\frac{1}{\left(\tau-\alpha^{*}\right)} \frac{\mathrm{d} \alpha^{*}}{\mathrm{~d} r}-\frac{1}{(\tau-\beta)} \frac{\mathrm{d} \beta}{\mathrm{d} r}-\frac{1}{\left(\tau-\beta^{*}\right)} \frac{\mathrm{d} \beta^{*}}{\mathrm{~d} r} \\
& +\mathrm{i} \sum_{\mathrm{j}=1}^{3}\left(\frac{1}{\tau-\tau_{\mathrm{j}}-\mathrm{i} r}-\frac{1}{\tau-\tau_{\mathrm{j}}+\mathrm{i} r}\right)
\end{aligned}
$$

Then

$$
A_{4}=\frac{\mathrm{i}}{2} \frac{1}{\rho} \frac{\mathrm{d} \rho}{\mathrm{d} r} \hat{r} \cdot \vec{\tau}
$$

so the Skyrme field from the integration along time lines would give

$$
f(r)=-\frac{1}{2} \int_{-\infty}^{\infty} \frac{1}{\rho} \frac{\mathrm{d} \rho}{\mathrm{d} r} \mathrm{~d} \tau-\pi
$$

The $\pi$ comes from the difference between instantons on $S^{4}$ and $\mathbb{R}^{4}$, the integration along time lines in $\mathbb{R}^{4}$ must be closed with a semi-circle at infinity to give a truly closed integration contour. With this addition the boundary condition $\left.U\right|_{\infty} \rightarrow 1$ is satisfied. The integral (209) may be computed using standard techniques of contour integration, yielding 


$$
\begin{aligned}
f(r) & =\pi \mathrm{i} \frac{\mathrm{d}}{\mathrm{d} r}(\alpha+\beta)+2 \pi \\
& =\pi \mathrm{i} \frac{\mathrm{d}}{\mathrm{d} r}(\Im(\alpha)+\Im(\beta))+2 \pi
\end{aligned}
$$

using $\alpha+\alpha^{*}+\beta+\beta^{*}$ is independent of $r$. The roots of a quartic can be found in closed form, however this does not elucidate the properties of the configuration.

The JNR potential has 5 parameters, $\tau_{\mathrm{i}}$ and the 2 independent ratios of the $\lambda_{\mathrm{i}}$. $\tau_{3}$ can be varied arbitrarily by a transformation moving along the line of cubics (changing the porism of triangles), leaving 4 parameters. We will call these $T_{1}$, $T_{2}, \Lambda_{1}$ and $\Lambda_{2}$ in the equivalent 't Hooft parametrization. (Take $\lambda_{3}=1, \tau_{3}=0$ and go to the 't Hooft parametrization.) A rigid translation of the $T_{\mathrm{i}}$ 's does nothing hence we take $T_{1}=-T$ and $T_{2}=T$, leaving 3 parameters. Finally imposing time reversal symmetry $\Lambda_{1}=\Lambda_{2}=\Lambda$, we get

$$
N(r, \tau)=\left(r^{2}+\tau^{2}\right)^{2}+2\left(\Lambda+T^{2}\right) r^{2}+2\left(\Lambda-T^{2}\right) \tau^{2}+T^{4}+2 \Lambda T^{2}
$$

which implies that $\Im(\alpha)=\Im(\beta)$ and since this is just a quadratic equation in $\tau^{2}$

$$
(\Im(\alpha))^{2}=\frac{1}{2}\left(R^{2}+\Lambda-T^{2}+\left(\left(r^{2}+\Lambda+T^{2}\right)-\Lambda^{2}\right)^{\frac{1}{2}}\right)
$$

and

$$
f(r)=2 \pi-2 \pi \frac{\mathrm{d}}{\mathrm{d} r} \Im(\alpha)
$$

As $T \rightarrow \infty$ we can find the form of $\alpha$, yielding

$$
f(r)=2 \pi\left(1-\left(1+\frac{\Lambda}{r^{2}}\right)^{-\frac{1}{2}}\right)
$$

This has energy $E=1.855535 \times 24 \pi^{2}$ when $\Lambda=2.6211$. Actually the true minimum occurs for $T^{2} \approx 84.6$ and $\Lambda=2.6427$, with $E=1.855529 \times 24 \pi^{2}$ which has been shown numerically (see [31]).

The time centered 't Hooft potential

$$
\rho=1+\frac{\Lambda+\Delta}{r^{2}+(\tau-T)^{2}}+\frac{\Lambda-\Delta}{r^{2}+(\tau+T)^{2}}
$$


gives an approximate potential

$$
f(r)=2 \pi-\pi\left(1+\frac{\Lambda+\Delta}{r^{2}}\right)^{-\frac{1}{2}}-\pi\left(1+\frac{\Lambda-\Delta}{r^{2}}\right)^{-\frac{1}{2}}
$$

which is the product of two $B=1$ hedgehogs of scale parameters $\Lambda+\Delta$ and $\Lambda-\Delta$. The minimum occurs at $\Delta=0, \Lambda=2.6211$ with $E=1.855536 \times 24 \pi^{2}$. The energy is symmetric in $\Delta$ so it is a reasonable conjecture that the minimum actually occurs at $\Delta=0$.

The spherically symmetric $B=2$ hedgehog is stable against perturbations preserving that symmetry. Its unstable modes violates this symmetry and span a 6 dimensional vector space. Under $\mathrm{O}(3)$ rotations these decompose into two 3-dimensional irreducible sub-spaces, $\mathrm{O}(3)$ is vectorial for one and axial for the other.

In terms of the product ansatz we lower the energy if two coincident Skyrmions are displaced - along 3 independent axes (axial mode) or rotated relatively in iso-space about 3 independent axes (vectorial mode). There are actually 3 modes which increase the energy while preserving the hedgehog form, the 3 independent parameters found above, $\Lambda_{1}, \Lambda_{2}$ and $T$. There are 6 zero modes corresponding to translations and global rotations (equivalently iso-rotations) and including the 6 modes which decrease the energy - unstable or negative modes, giving a total of 15 modes. Using the JNR parametrization the minimum energy hedgehog has

$$
\rho=\frac{1}{r^{2}+\tau^{2}}+\frac{\Lambda^{\prime}}{r^{2}+\left(\tau-T^{\prime}\right)^{2}}+\frac{\Lambda^{\prime}}{r^{2}+\left(\tau+T^{\prime}\right)^{2}}
$$

where $\Lambda^{\prime}=\frac{\Lambda}{T^{2}}$ and $T^{\prime}=\left(T^{2}+2 \Lambda\right)^{\frac{1}{2}}$ and with $T^{2}=84.6, \Lambda=2.6427$.

This configuration corresponds to a line of cubics with collinear roots. The perturbations which break the collinearity gives the vector instability while the perturbations that rotate the line gives the axial one. These perturbations of the instanton configuration give rise to exactly the same perturbations of the corresponding Skyrmions which reduce the energy according to the analysis of Bang and Wirzba[51]. We will return to these unstable modes in section 4.3.

The most attractive channel instantons and hence Skyrmions, in the Hartshorne description, are distinguished by concentric circles and ellipses. For high eccentricities we have two well separated Skyrmions with relative iso-rotation of $180^{\circ}$. The minimum energy configuration appears when the ellipse degenerates to a circle of radius $\frac{R}{2}$ (see figure 10). Evidently the porism of triangles is given by a set of equilateral triangles, obtained from one another by a simple 


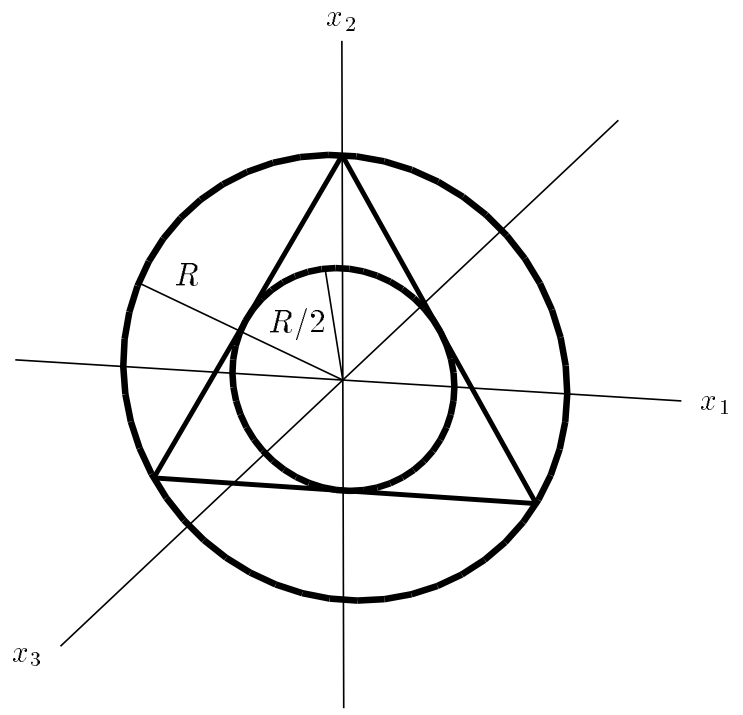

Fig. 10. Reflection symmetry: $x_{1} \rightarrow-x_{1}$ and $x_{2} \rightarrow-x_{2}$, and $\mathrm{O}(2)$ symmetry about the $x_{3}$ axis.

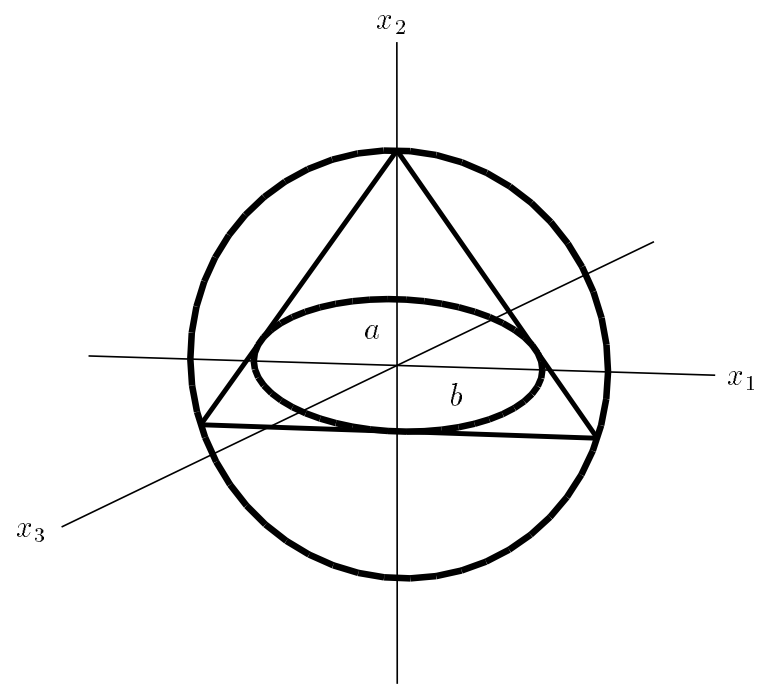

Fig. 11. Reflection symmetry about the three orthgonal axes: $x_{\mathrm{i}} \rightarrow-x_{\mathrm{i}}$.

rotation. This Hartshorne configuration exudes $\mathrm{O}(2)$ symmetry, which indeed the instanton configuration, and the subsequent Skyrmion field also exhibit.

If the Hartshorne ellipse is only concentric with the circle then the $\mathrm{O}(2)$ symmetry reduces to reflection symmetry with respect to the three axes. The Poncelet condition requires that $a+b=R$, where $a$ and $b$ are the semi-major and semi-minor axes respectively while $R$ is the radius of the circle (see figure 11). When the ellipse becomes extremely eccentric (but always remains concentric with the circle), it is easy to verify that the configuration corresponds 


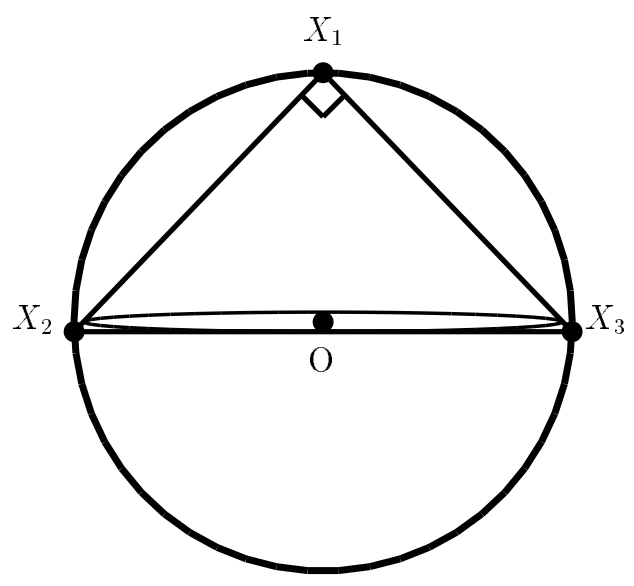

Fig. 12. Hartshorne diagram corresponding to two well separated Skyrmions in the most attractive channel.

to well separated Skyrmions with a relative iso-rotation of $180^{\circ}$. Indeed the triangle degenerates to a right isoceles triangle with hypotenuse approximately a diameter (see figure 12). The configuration corresponds to Skyrmions localized at positions $X_{2}$ and $X_{3}$ with iso-rotation factors $\left(X_{2} \widehat{-} X_{1}\right) \cdot \vec{\tau}$ and $\left(X_{3} \widehat{-} X_{1}\right) \cdot \vec{\tau}$ respectively $\left(\left(X_{2}-X_{1}\right)\right.$ is a unit vector in the $X_{2}-X_{1}$ direction $)$. The configuration is

$$
\begin{aligned}
U=\left(\mathrm { i } \left(X_{2} \widehat{-}\right.\right. & \left.\left.X_{1}\right) \cdot \vec{\tau}\right)^{\dagger} U\left(x-X_{2}\right) \mathrm{i}\left(X_{2} \widehat{-} X_{1}\right) \cdot \vec{\tau} \\
& \times\left(\mathrm{i}\left(X_{3} \widehat{-} X_{1}\right) \cdot \vec{\tau}\right)^{\dagger} U\left(x-X_{3}\right) \mathrm{i}\left(X_{3} \widehat{-} X_{1}\right) \cdot \vec{\tau}
\end{aligned}
$$

where $U\left(x-X_{2}\right)$ is a Skyrmion at $X_{2}$, hence the relative iso-orientation is

$$
\begin{aligned}
R & =\mathrm{i}\left(X_{2} \widehat{-} X_{1}\right) \cdot \vec{\tau}(-\mathrm{i})\left(X_{3} \widehat{-} X_{1}\right) \cdot \vec{\tau} \\
& =\mathrm{i}\left(\left(X_{2} \widehat{-} X_{1}\right) \times\left(X_{3} \widehat{-} X_{1}\right)\right) \cdot \vec{\tau}
\end{aligned}
$$

for the Skyrmion located at $X_{3}$. However the vector

$$
\left(X_{2} \widehat{-} X_{1}\right) \times\left(X_{3} \widehat{-} X_{1}\right)
$$

is a unit vector (because the angle at $X_{1}$ is $90^{\circ}$ ), orthogonal to the plane defined by $X_{1}, X_{2}$ and $X_{3}$, and hence also orthogonal to the separation axis $X_{2}-X_{3}$. Furthermore since $R$ is of the form $\mathrm{i} \hat{n} \cdot \vec{\tau}$ it effects an iso-rotation by exactly $180^{\circ}$ as desired, about the axis $\left(X_{2}-X_{1}\right) \times\left(X_{3}-X_{1}\right)$. The discrete symmetries of the concentric ellipse expand to the continuous $\mathrm{O}(2)$ symmetry of the concentric circles and implies that this configuration should correspond 
to the minimum energy configuration which is known to have toroidal symmetry [52,53,54]. After minimization with respect to the (1) free parameter $R$ one actually finds the minimal torus to within a few percents.

The manifold of attractive channel Skyrme fields forms an 11 dimensional submanifold of the full 15 dimensional manifold of instanton generated Skyrme fields. The ellipse simply has 2 degrees of freedom, its eccentricity and its orientation, instead of 4 , and the orientation of the 2-plane must be orthogonal to the time axis implying only the action of $\mathrm{O}(4) /(\mathrm{O}(2) \times \mathrm{O}(2))$ yielding $6-(1+1)=4$ parameters instead of 6 , reducing by another 2 . Hence 15 goes to 11 parameters. As 9 parameters correspond to the action of the global symmetry group of translations, rotations and iso-rotations, we must find the gradient flow curves in a two dimensional subspace, parametrized by $a, b$ and $R$ subject to the constraint $a+b=R$. These gradient flow curves would start approximately at the asymptotic critical point of the two infinitely separated, minimal energy, isolated Skyrmions, and arriving at the minimal energy toroidal configuration. Since the product ansatz tells us that asymptotically this is a 10 dimensional manifold, the gradient flow must also yield a 10 dimensional manifold. This is intuitively reasonable, minimizing in a two dimensional manifold will typically yield a one dimensional "valley" or "path" of steepest descent linking together the critical points. Hence we do indeed obtain a $9+1=10$ dimensional manifold of most attractive channel instanton generated Skyrme fields.

The gradient flow has not to date been calculated. Hosaka et al [55] have exhibited a qualitatively similar manifold of constrained minima. It is obtained simply by letting the set of triangles vary from that of the right isoceles triangle corresponding to widely separated Skyrmions, to the equilateral triangle of the toroid through intermediate symmetric isoceles triangles. The Hartshorne ellipse starts at very high eccentricity and varies until it degenerates to the circle. The energy is minimized for each intermediate triangle, fixing the value of $R$. The minimal energy for fixed eccentricity decreases monotonically until the circle is reached at $a / b=1$. The constrained energies are always within $1 \%$ or $2 \%$ of the similar but fully numerical computations of Verbaarschot et al 56] and of Walhout 57].

Geometrically the 10 dimensional most attractive channel manifold consists of the direct product of a 6 dimensional (global) manifold, which is generated by 3 independent spatial translations and 3 independent isospin rotations, with a 4 dimensional (relative) manifold. The 4 dimensional relative manifold consists of "centered" Skyrme fields, which can be acted upon by the group of spatial rotations. The manifold is parametrized by a coordinate depicting separation and 3 angular coordinates taken without loss of generality to be the Euler angles specifying a frame of unoriented Cartesian axes. The generic $\mathrm{SO}(3)$ orbit is actually only $\mathrm{SO}(3) / V$, where $V$ is the group of $180^{\circ}$ rotations about 
the 3 axes (and the identity), which of course leave an unoriented Cartesian frame invariant. At minimal separation the orbit degenerates to $\mathbb{R} P_{2}$ which is the same as the sphere $S^{2}$ with antipodal points identified, physically it is the orbit of the symmetry axis of the toroid, taking into account that this axis is unoriented.

The Atiyah-Hitchin manifold [58], corresponding to the moduli space of centered 2 BPS-monopole configurations has exactly the same orbit structure. Hence the Atiyah-Hitchin manifold is a good candidate describing centered, attractive channel Skyrmions. The Atiyah-Hitchin manifold has an implicit metric, the Atiyah-Hitchin metric. This metric, however, is not appropriate for Skyrmions. The Atiyah-Hitchin encodes in it velocity-dependent Coulomb interactions between monopoles, which are absent for Skyrmions. The true metric must be calculated using the Skyrme energy functional. This has been done by Leese et al [59].

\subsection{Rational maps and multi-baryon number states of the Skyrme model}

The study of high baryon number solutions of the Skyrme model has always been a very difficult problem to tackle. What is lacking is a good ansatz which captures the symmetries and simplifies the equations of motion.

A first glance at higher baryon number was given by Braaten et al [60] almost 10 years ago. Using relaxation methods on a highly (in those days) powerful Cray super-computer, they isolated states for the sectors $B=2$ to $B=6$ and computed their energies. Quite surprisingly, the configurations for $B=3,4,5$ and 6 took very geometrical shapes, tetrahedral $(B=3)$, octahedral $(B=4)$, and less symmetrical ones for $B=5$ and 6 . Obviously, these look nothing like nuclei as described by shell or droplet models from traditional nuclear physics. Of course, the doughnut shaped $B=2$ state, the Skyrme model's deuteron state, looks very little like a pair of weakly interacting nucleons. However, as the classical binding energy of the doughnut $(100 \mathrm{MeV})$ is much greater than the deuteron's real binding energy (around $2 \mathrm{MeV}$ ), one could hope that the $B=2$ case is not typical for the Skyrme model and that things would settle down for higher baryon number. We will see that it is not the case, and one gets the impression from the literature that the results for $\mathrm{B}=2$ to 6 were both unexpected and not understood: consequently they were left pretty much

alone for the interim. In 1996 Battye and Sutcliffe [61] confirmed these results (except the $B=6$ state which seems to have been misidentified) using stateof-the-art software and hardware, as well as found the structure of probably minimum energy states with $B=7,8$ and 9 (see figure 13 taken from reference [61]). This indicates that indeed the Skyrme model favours regular geometric configurations instead of shell model-type lumps. These shapes in fact, are 

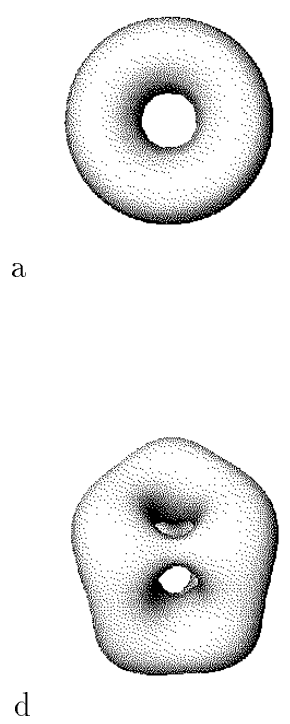

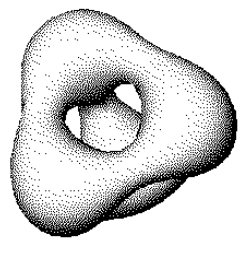

$\mathrm{b}$

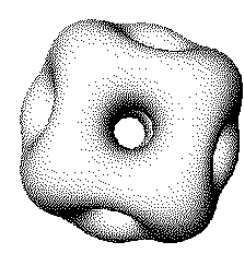

e

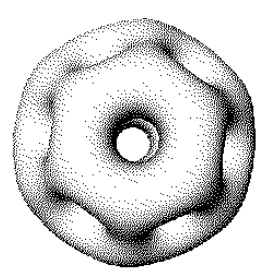

g

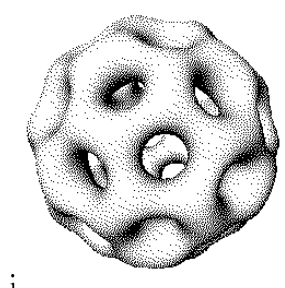

i

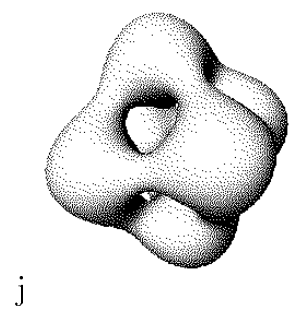

h
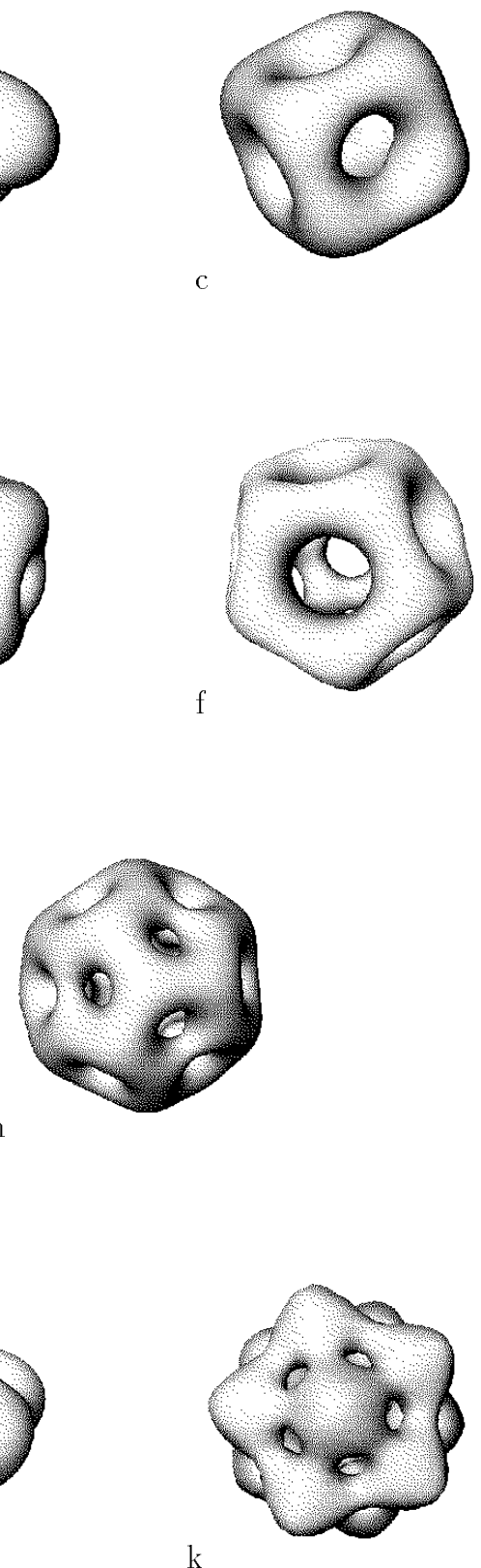

$\mathrm{k}$

Fig. 13. Various baryonic density for Skyrme states of baryon number 2 to 12 .

very similar to those found in chemistry. Only time and further investigations will tell if indeed and why such configurations are physically relevant.

One must note here that all these states have been found using numerical relaxation methods, with the algorithm feeling its way down the slopes of configuration space in search of the lowest energy possible in each given baryon number sector. Of course, it is impossible to be absolutely sure that the configurations obtained so far are absolute minima of the energy in each baryon 
number sector, and not merely local minima, without using other methods. Thus there is still a possibility that the spectrum of real states might be different, even though the fact that the energy per baryon number is very close to the Bogomolny bound (about $10 \%$ or so over the bound) makes this possibility quite remote. Oscillations and perturbations around those solutions have been considerd to test (at least locally) the stability of the solutions, and to compare the resulting spectrum with known excited states of nuclei. So far the results look encouraging but there is still a lot of work to be done before comparison with nature can be made in a serious manner 62,63.

As impressive as these numerical results might look, they are still a long way ahead of actually doing simulations of Skyrmion scattering processes for several initial Skyrmions or for large energy. Even though it actually is possible to do so, it still takes strong numerical skills to do the simulation and extract and interpret the results. To be able to push forward the current study of the model, and also to avoid that the study of soliton scattering is absorbed into purely numerical or computational physics, one needs some kind of analytical handle on the problem, even an approximate one (for a flavour of the difficulties encountered in the study of soliton-soliton scattering see the article by Crutchfield and Bell [64]). The instanton method which we have just described does exactly this but it is of little help for the study of large baryon number solutions, except under very special conditions 32]. The gradient flow curve method which we will present in section 4 does not help much either since to apply it we need to know the manifold connecting the critical points of the system for low energy in a given baryon number sector. However we do not know this manifold, and we actually deduce its structure from numerical simulations.

Help comes our way from the study of the BPS monopole system. It is not our intention to discuss this system here (see the review by Sutcliffe[65]). Pioneers of this model are Bogomolgny 66], Prasad and Sommerfield 67, 't Hooft 68, Polyakov 69, Atiyah and Hitchin 70, 71, Manton 72, Gibbons 38, Ward 73] and Nahm[74], to mention a few. The BPS monopole is a topological soliton of a massless Higgs type model with an $\mathrm{SU}(2)$ gauge symmetry, which saturates the Bogomolny bound of the theory [75,76]. Over the years, the spectrum of the model has been studied, and states with each value of the magnetic charge (which represents the winding number associated to the soliton in this model) have been isolated. Quite surprisingly, there exists a state with magnetic charge $N$ with symmetries identical to those of the Skryme model for baryon number $N$ [34] (this fact has been verified for $N$ ranging from 1 to 9 ): toroidal for $N=2$, tetrahedral for $N=3$, etc. This of course does not indicate that BPS fields should be used to study baryonic systems, but it does indicate that the mathematical arsenal used in the study of the 
BPS system could perhaps be generalized to Skyrmion systems ${ }^{2}$. The tools of interest here are called rational maps and were introduced in the study of BPS systems by Donaldson[77], and recently elaborated upon by Jarvis 78]. We will not explain in detail the method nor show how it works. Instead we will give a taste of how it can be applied to Skyrmion systems following the article of Houghton et al [34.

BPS monopoles are solutions of a model possessing a symmetry breaking term which breaks an SU(2) symmetry via a triplet of Higgs fields to a U(1) symmetry, interpreted as electromagnetism. Inside the monopole, the $\mathrm{SU}(2)$ symmetry is intact, but it has to be broken to U(1) on the outside so as to give the soliton finite energy (because of a potential term in the Lagrangian density). Restricting the Higgs field to its broken symmetry value outside the soliton fixes its length, but not its direction: it can take any direction in $\mathbb{R}^{3}$ and in fact describes an $S^{2}$. Infinity in flat 3-dimensional space also consists of a 2sphere, so the Higgs field of the monopole is a map from $S^{2}$ to $S^{2}$. Such maps are divided in disjoint homotopy classes numbered by the number of times the first $S^{2}$ is wrapped around the second $S^{2}$. This topological winding number is proportional to the magnetic charge of the field. The vacuum has the Higgs field pointing the same way everywhere (thereby having winding number and magnetic charge 0), while the unit magnetic charge monopole looks like a hedgehog from afar. By stereographic projection one can transform the map from $S^{2}$ to $S^{2}$ to another which maps the complex plane in another complex plane (if we identify a given point of the spheres with infinity in the usual way). Donaldson 77] showed that there is a one to one relation between the field of an $N$ monopole and rational maps $R(Z)$ of degree N. A rational map of degree $N$ from $\mathbb{C} \rightarrow \mathbb{C}$ is defined as:

$$
Z \mapsto R(Z)=\frac{p(Z)}{q(Z)}
$$

where $p(Z)$ and $q(Z)$ are polynomials of at most degree $N$, with at least one being of degree $N$ and with no common roots. The parameters of the polynomials generate (much like in the case of the parameters of the instantons of the previous subsection) a finite dimensional manifold of configurations and can be chosen so as to give the soliton some desired symmetries, and fine tuned to lower the energy as much as possible. We refer the reader to the literature for further details 34]. Experience shows that once a particular set of symmetries

\footnotetext{
${ }^{2}$ In fact, the similarity between BPS and Skyrme systems could run deeper than it appears. During his talk at the CRM-Fields-CAP 1997 workshop "Solitons" in Kingston, Canada, N.S. Manton conjectured that the moduli space of vortices is a submanifold of the moduli space of BPS monopoles, which is itself a submanifold of that of Skyrmions, itself being included in the moduli space of $\mathrm{SU}(2)$ instantons. This fascinating conjecture remains to be established.
} 


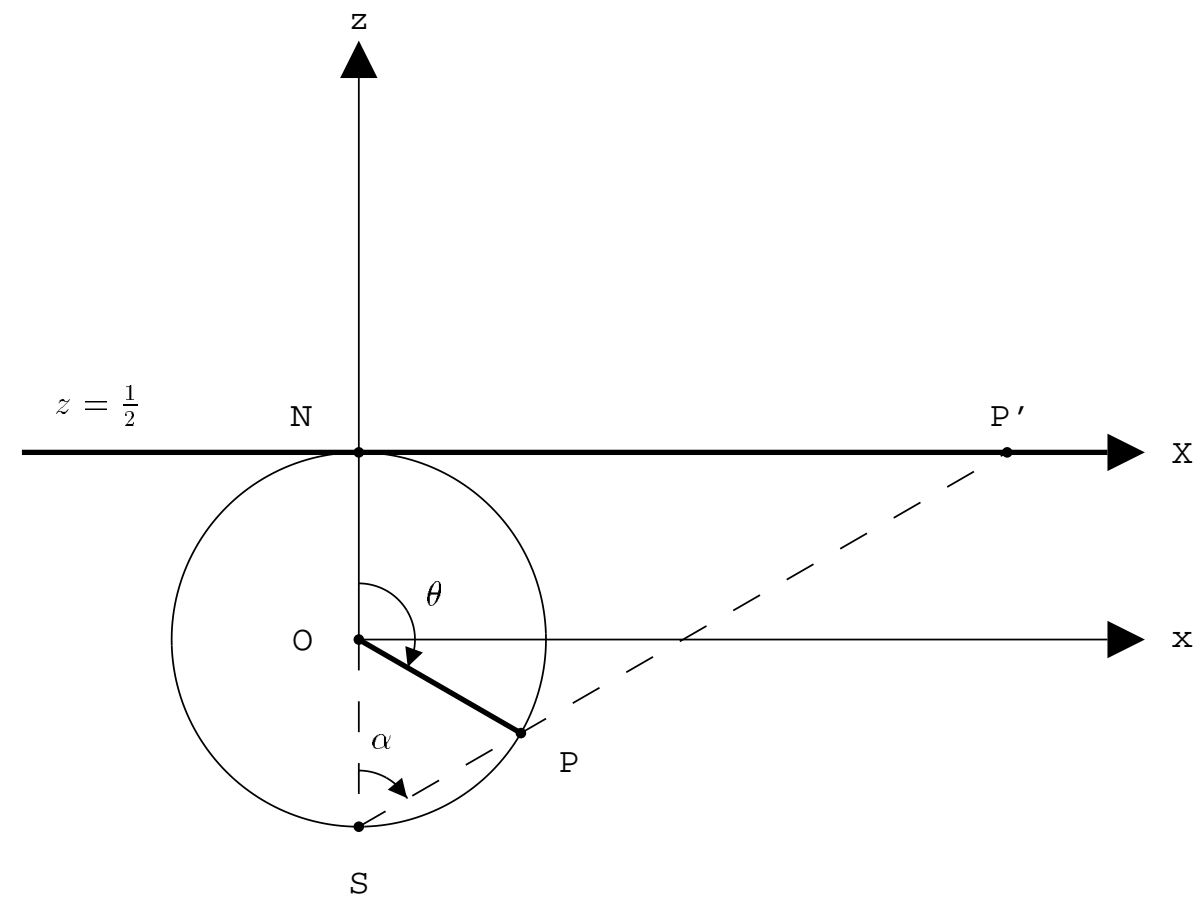

Fig. 14. Stereographic projection of an $S^{2}$ of radius $1 / 2$ on plane $(X Y)$. It gives $Z=X+\mathrm{i} Y=\tan (\theta / 2) \mathrm{e}^{\mathrm{i} \phi}$, and the projection angle $\alpha=\theta / 2$.

has been chosen and implemented in the rational map, the method gives a good approximation to the exact (numerical) result.

Houghton et al [34] generalized this method to the Skyrme model by choosing the following ansatz for Skyrme fields:

$$
U(\vec{x})=e^{\mathrm{if}(\mathrm{r}) \hat{\mathrm{n}}_{\mathrm{R}(\mathrm{Z})} \cdot \vec{\tau}}
$$

where $\hat{n}_{\mathrm{R}(\mathrm{z})}$ is a function of $Z$, or in other words of the usual spherical angles $\theta$ and $\phi$ only. $f(r)$ depends solely on the distance $r$ from the origin. This ansatz is interesting in that it singles out the distance to the origin from the angular coordinates. As Houghton et al noted, one can understand this ansatz as mapping the two-spheres centered on the origin of space onto the 2-spheres which correspond to latitudes in the $S^{3}$ (of SU(2)). To do this the two-spheres of the $\mathbb{R}^{3}$ of space are mapped via stereographic projection onto the complex plane (with complex infinity identified to a single point of the sphere) as shown in figure 14. Elements $Z$ of this plane are then functions of the spherical angles $\theta$ and $\phi$ :

$$
Z=\tan (\theta / 2) \mathrm{e}^{\mathrm{i} \phi}
$$

We are now on familiar ground: elements of the initial complex plane $Z$ are 
then mapped by the rational map $R(Z)$ into another complex plane, itself obtained by stereographic projection of the latitudes of the $\mathrm{SU}(2)$ three-sphere:

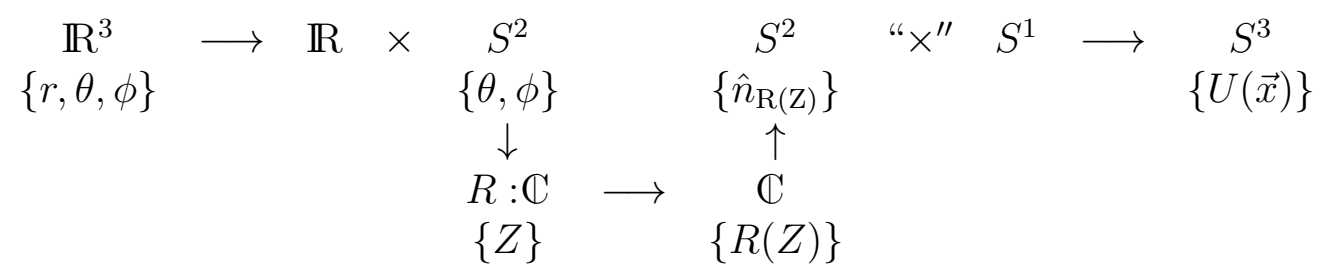

$\hat{n}_{R(Z)}$ is expressed as follows:

$$
\hat{n}_{\mathrm{R}(\mathrm{Z})}=\frac{1}{\left.|1+| R\right|^{2} \mid}\left(2 \Re(R), 2 \Im(R), 1-|R|^{2}\right)
$$

where $\Re(R(Z))$ and $\Im(R(Z))$ represent the real and imaginary parts of $R(Z)$ respectively. $\hat{n}_{\mathrm{R}(\mathrm{Z})}$ depends on the rational map $R(Z)$ chosen, and gives, for example, the ordinary radial vector $\hat{n}$ if $R(Z)=Z$. This particular map reproduces of course the ordinary Skyrmion.

The parametrization (222) chosen for $U$ can seem a bit restrictive. It is especially suited to reproduce configurations localized around the origin. It probably does not reproduce accurately, well separated Skyrmions (although it works surprisingly well and gives an idea of some processes). The main advantage of this parametrization is that it decouples contributions from the radial (i.e. ones related to $f(r)$ ) and from the angular part (i.e. related to $R(Z)$ ). This way, it is possible to first impose a given symmetry to the configuration and then minimize the angular contribution to the energy. Subsequently by minimizing with respect to $f(r)$ we obtain the minimum energy possible within the ansatz. We note that in certain cases, varying the value of some parameters of $R(Z)$ gradually and minimizing with respect to $f(r)$ gives "snapshots" of certain scattering processes. The case of $B=3$ is a good example.

Choosing the parameters in the polynomials $p(Z)$ and $q(Z)$ so as to get solutions with the right symmetries is a technical but important point which we will illustrate by the following example. Let us compute the expression for the rational map $R(Z)$ which gives the Skyrmion field $U(\vec{x})=u_{0}+\mathrm{i} \vec{u} \cdot \vec{\tau}$ with lowest energy in the $B=2$ sector. As we will see in section 4.3 the following three symmetries are characteristic of a pair of Skyrmions converging together at the origin to form a tightly bound minimum energy toroidal solution of the equations of motion:

$$
\begin{aligned}
I_{1}: x \rightarrow-x: & u^{1} \rightarrow u^{1} \\
y \rightarrow y & u^{2} \rightarrow-u^{2} \\
z \rightarrow z & u^{3} \rightarrow u^{3}
\end{aligned}
$$




$$
\begin{aligned}
I_{2}: x \rightarrow x: & u^{1} \rightarrow u^{1} \\
y \rightarrow-y & u^{2} \rightarrow-u^{2} \\
z \rightarrow z & u^{3} \rightarrow u^{3} \\
& \\
& \\
I_{3}: x \rightarrow x: & u^{1} \rightarrow u^{1} \\
y \rightarrow y & u^{2} \rightarrow u^{2} \\
z \rightarrow-z & u^{3} \rightarrow-u^{3} .
\end{aligned}
$$

The most general expression for the rational map $R$ of degree 2 is given by:

$$
R(Z)=\frac{\alpha Z^{2}+\beta Z+\gamma}{\lambda Z^{2}+\mu Z+\nu}
$$

where $\alpha, \beta, \gamma, \lambda, \mu$ and $\nu$ are constants (real or imaginary) to be determined by imposing the symmetries $I_{1}, I_{2}$ and $I_{3}$. To do this, we need to parametrize them using $Z$ and $R(Z)$. Using (223) and

$$
\vec{u}=\sin f(r) \hat{n}_{\mathrm{R}(\mathrm{Z})}
$$

we see that the transformation $x \rightarrow-x$ of $I_{1}$ is equivalent to $Z \rightarrow-\bar{Z}$ after projection on the complex plane. Similarly, $y \rightarrow-y$ and $z \rightarrow-z$ just translate to $Z \rightarrow \bar{Z}$ and $Z \rightarrow 1 / \bar{Z}$, respectively. For our needs we will only consider the new transformations $\mathcal{I}_{1} \equiv I_{1} I_{2}$ and $\mathcal{I}_{2} \equiv I_{2} I_{3}$ (the product $I_{3} I_{1}$ being discarded since it brings no new constraint on $R$ ). The symmetry $\mathcal{I}_{1}$ is given then by:

$$
\begin{aligned}
\mathcal{I}_{1}: Z \rightarrow-Z: & \hat{n}^{1} \rightarrow \hat{n}^{1} \\
: & \hat{n}^{2} \rightarrow \hat{n}^{2} \\
: & \hat{n}^{3} \rightarrow \hat{n}^{3} .
\end{aligned}
$$

The field $\hat{n}_{\mathrm{R}(\mathrm{Z})}$ being then invariant under the transformation, this imposes the constraint

$$
R(-Z)=R(Z)
$$

on $R$. Similarly, $\mathcal{I}_{2}$ is written as:

$$
\begin{aligned}
\mathcal{I}_{2}: Z \rightarrow 1 / Z: & \hat{n}^{1} \rightarrow \hat{n}^{1} \\
: & \hat{n}^{2} \rightarrow-\hat{n}^{2} \\
: & \hat{n}^{3} \rightarrow-\hat{n}^{3} .
\end{aligned}
$$


A short calculation shows that $\hat{n}$ transforms under this transformation like $\hat{n}_{\mathrm{R}(\mathrm{Z})} \rightarrow \hat{n}_{1 / \mathrm{R}(\mathrm{Z})}$. So $R$ also has to satisfy

$$
R(1 / Z)=1 / R(Z)
$$

We now apply the conditions (232) and (234) to the general form of $R$, 229) to fix the constants $\alpha, \beta, \gamma, \lambda, \mu$ and $\nu$. Equation (232) sets $\beta$ and $\mu$ to 0 , while (234) gives the following constraints on the remaining parameters:

$$
\alpha \gamma=\nu \lambda \quad \text { and } \quad \alpha^{2}+\gamma^{2}=\lambda^{2}+\nu^{2} .
$$

We can set $\nu=1$ by scaling each variable then we find:

$$
R(Z)=\frac{Z^{2}-\gamma}{-\gamma Z^{2}+1} \text { or } \quad \frac{-Z^{2}-\gamma}{\gamma Z^{2}+1}
$$

The second possibility corresponds to a rotation by $90^{\circ}, Z \rightarrow \mathrm{i} Z$. One then replaces this expression in the energy of the ansatz (222) given by

$$
E=4 \pi \int\left(r^{2} f^{\prime 2}+2 N\left(f^{\prime 2}+1\right) \sin ^{2} f+\mathcal{I} \frac{\sin ^{4} f}{r^{2}}\right) \mathrm{d} r
$$

where

$$
\mathcal{I}=\frac{1}{4 \pi} \int\left(\frac{1+|Z|^{2}}{1+|R|^{2}}\left|\frac{\mathrm{d} R}{\mathrm{~d} Z}\right|\right)^{4} \frac{2 \mathrm{i} \mathrm{d} Z \mathrm{~d} \bar{Z}}{\left(1+|Z|^{2}\right)^{2}} .
$$

As seen earlier, the contribution to the energy coming from the angular part of the ansatz decouples from the radial part, and $\mathcal{I}$ can be minimized as a function of the parameter $\gamma$. One finds the extremal value $\mathcal{I}=\pi+8 / 3$ for $\gamma=0$. This simply corresponds to the map $R(Z)=Z^{2}$ which possesses the cylindrical symmetry

$$
R\left(\mathrm{e}^{\mathrm{i} \alpha}\right)=\mathrm{e}^{2 \mathrm{i} \alpha} R(Z)
$$

which contains the discrete symmetries $I_{1}$ and $I_{2}$. This is the toroidal configuration which we will discuss more when we describe the low energy manifold for the sector $B=2$. Replacing the minimum value of $\mathcal{I}$ in the expression of the energy and minimizing further relative to the radial function $f$, one finally obtains a value only $3 \%$ greater than the mass of the torus obtained by a fully numerical computations. Rational maps with more complicated symmetries generalise the previous discussion and are presented in reference [34]. 
We will close this section with the following comments about the number of "holes" (regions with zero baryon number density) which are present in a given configuration. $\mathrm{dR} / \mathrm{dz}$ is zero if the Wronskian is zero

$$
W(z)=p^{\prime}(Z) q(Z)-p(Z) q^{\prime}(Z)=0
$$

which is the numerator of $\mathrm{d} R / \mathrm{dZ}$. The baryon number for the ansatz (222) is given by

$$
N=\frac{1}{4 \pi} \int\left[\left(\frac{1+|Z|^{2}}{1+|R|^{2}}\right)\left|\frac{\mathrm{d} R}{\mathrm{~d} Z}\right|\right]^{2} \frac{2 \mathrm{id} Z \mathrm{~d} \bar{Z}}{\left(1+|Z|^{2}\right)^{2}} .
$$

The baryon number density is then proportional to $\mathrm{d} R / \mathrm{dZ}$ and vanishes where the Wronskian is zero. This means that it will be zero along rays pointing to infinity from the origin, and whose directions are given by the roots of $W(Z)$. $R(Z)$ being of degree $N, W(Z)$ is generically of degree $2 N-2$ (the naively leading power of $Z$ cancels in (240)), and so a configuration of this baryon number should have $2 N-2$ holes in it. For the case $N=2, q(Z)=1$ so the Wronskian is in fact linear in $Z$ and only has one zero, which is consistent with the trivial fact that a torus only has one hole.

\subsection{Search for a sphaleron in the Skyrme model: Morse theory}

Morse theory [79,80, 81, 82], otherwise known as global variational analysis, relates the topology of a manifold to the number of and types of critical points of a function defined on the manifold. For application in field theory, the manifold in question is the (infinite dimensional) space of all field configurations and the "function" defined on this manifold is generally a functional, typically the energy functional or perhaps the action functional. The generalization of Morse theory to the infinite dimensional arena goes under the name of Ljusternik-Snirelman theory [83].

The classic, illustrative example of the application of Morse theory is furnished by a function defined on a torus. We take the outer radius to be $R$ and the inner radius to be $r<R$, with the symmetry axis pointing along the $x$ axis. The function in question should be a "Morse function", the definition of which we will address below. We will consider the function defined by the value of the $z$ coordinate of the Cartesian coordinates of each point of the torus, which happens to be a Morse function. Physically, the function in question is the altitude from the $z=0$ plane for each point on the torus. Now let us consider the critical points of this function on the torus. It is well known that every function defined on a compact manifold achieves its global maximum 


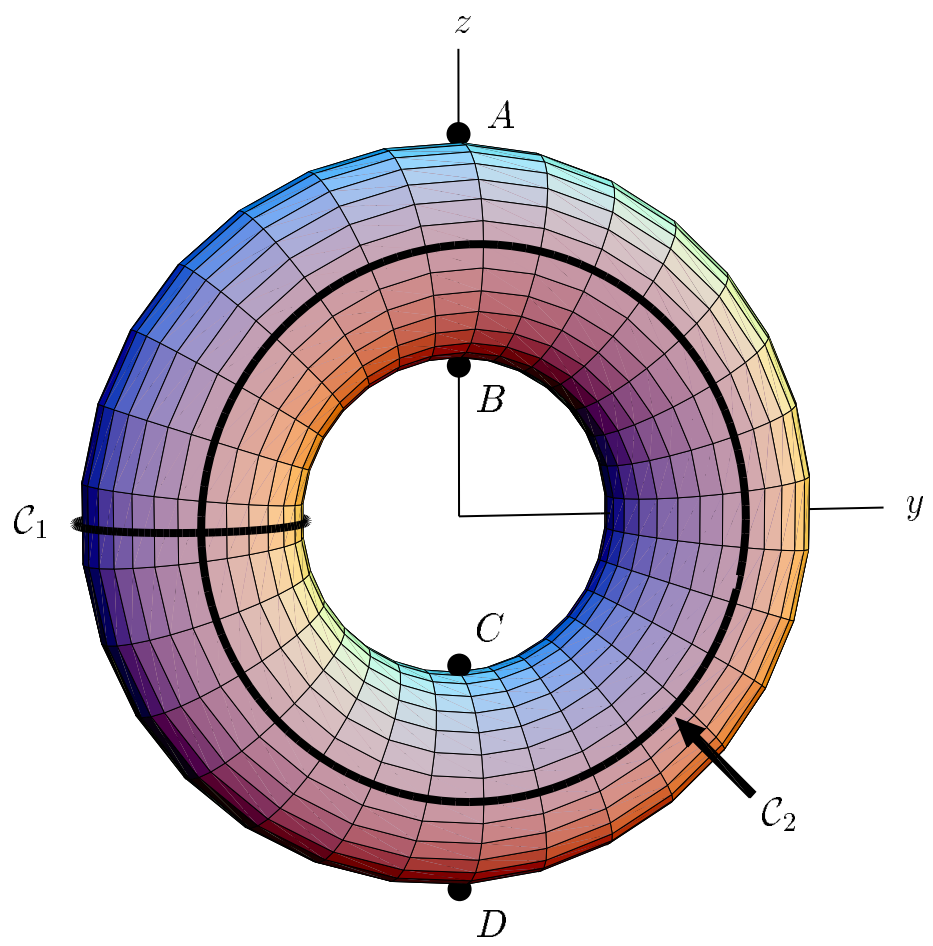

Fig. 15. The altitude fonction $f(x, y, z)=z$ defined on an ordinary torus possesses 4 critical points: the global maximum $A$, the global minimum $D$, and two minimax's $B$ and $C$. Morse theory identifies the presence of the minimaxima with the existence on the torus of two types of non-contractible loops $\mathcal{C}_{1}$ and $\mathcal{C}_{2}$.

and its global minimum somewhere on the manifold. Clearly the function will be critical at these two points, hence, in fact the compactness of the manifold, which is is a topological characterization, has implied the existence of two critical points. There are however, even more. These are predicted by Morse theory, due to the non-simple connectedness of the torus. The torus admits two different non-contractible closed loops (see figure [15). Morse theory implies that there are at least two other critical points, which are minimax's. Physically we can just see them: the points $B$ and $C$ in figure 15. If we consider the intersection of the torus with successive planes of constant $z$, the two planes for which the topological nature of this intersection changes correspond to the positions of the minimax's. Successively, as we sweep the plane through the torus the intersection (see figure 16) will commence as a point (a very degenerate "circle" or loop), then a normal loop, but then at one point the loop will pinch in and touch itself and then break up into two disjoint loops. This is the first minimax or saddle point. Then the two loops will separate a little but again come back and touch. When they touch is another saddle point, and further they will separate to form a single loop, which will finally terminate by degenerating to a point.

To prove the existence of an additional critical point due to non-trivial topol- 


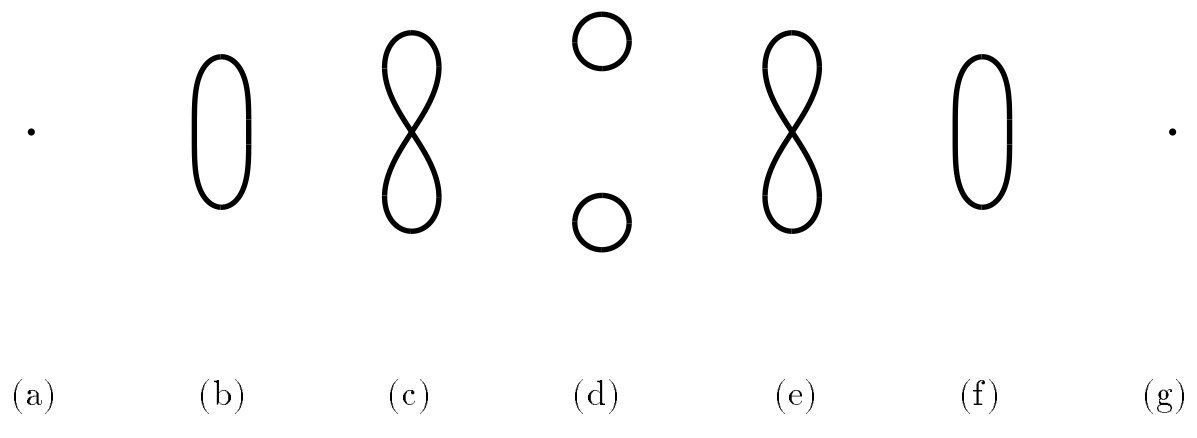

Fig. 16. Intersection of the torus with planes (a) $z=R+r$, (b) $z=R$, (c) $z=R-r$, (d) $z=0$, (e) $z=-R+r$, (f) $z=-R$ and (g) $z=-R-r$.

ogy, consider the somewhat more general example of a smooth function $f(x)$ defined on a compact manifold with a non-contractible loop. We wish to prove that there exist at least three critical points for the function. It is immediate that the global minimum $f\left(x_{\min }\right)$ and the global maximum $f\left(x_{\max }\right)$ exist. Without loss of generality $f\left(x_{\max }\right)>f\left(x_{\min }\right)$, since if $f\left(x_{\max }\right)=f\left(x_{\min }\right)$, the function is a constant. Also without loss of generality we may assume that the maximum and minimum are achieved as unique, individual, isolated points. This means that there are already two critical points hence if the minimum or maximum is achieved elsewhere there would be at least three critical points and we would be done.

Now consider the set of non-contractible loops in the manifold which go through the minimum. For each loop find the point at which the function is maximum, the set $\{\bar{x}\}$. If this occurs at several points for any one loop, choose the point $\bar{x}$ where $|\vec{\nabla} f|^{2}$ is minimal (since we are looking for a critical point where $|\vec{\nabla} f|=0)$. The maximum $f(\bar{x})$ is necessarily greater than $f\left(x_{\min }\right)$ since if it were not then $f(x)$ would have to be critical at $\bar{x}$, and then would already admit a third critical point at $\bar{x}$. Now we find the minimum of the set $\{f(\bar{x})\}$ by varying the loop. The point where this occurs must exist and will correspond to a critical point of the function, a saddle point. It must exist because a bounded, monotone, sequence in a compact manifold always admits a limit point. We consider the sequence of points obtained by finding $\bar{x}$ for a given curve and then varying successively the curve to yield $\bar{x}_{1}, \bar{x}_{2}$, etc. such that $f\left(\bar{x}_{\mathrm{i}}\right) \geq \bar{f}\left(x_{\mathrm{i}+1}\right)$ (see figure 17). Let $\bar{x}_{\mathrm{c}}$ be defined by $\bar{x}_{\mathrm{c}} \in\{\bar{x}\}$ such that $f\left(\bar{x}_{\mathrm{c}}\right)=\min (f(\bar{x}))$ and we call the curve passing through $\bar{x}_{\mathrm{c}}$ the critical curve, $\vec{x}_{\mathrm{c}}(t) . \vec{\nabla} f\left(\bar{x}_{\mathrm{c}}\right)$ must vanish. If $\left|\vec{\nabla} f\left(\bar{x}_{\mathrm{c}}\right)\right|>\delta(\delta>0)$ then consider the curve obtained by deforming the points of the critical curve in a neighborhood of $\bar{x}_{\mathrm{c}}$ along the direction opposite to the gradient:

$$
\vec{x}_{\mathrm{c}}(t) \rightarrow \vec{x}_{\mathrm{d}}(t)=\vec{x}_{\mathrm{c}}(t)-\epsilon(t) \vec{\nabla} f\left(\vec{x}_{\mathrm{c}}(t)\right),
$$




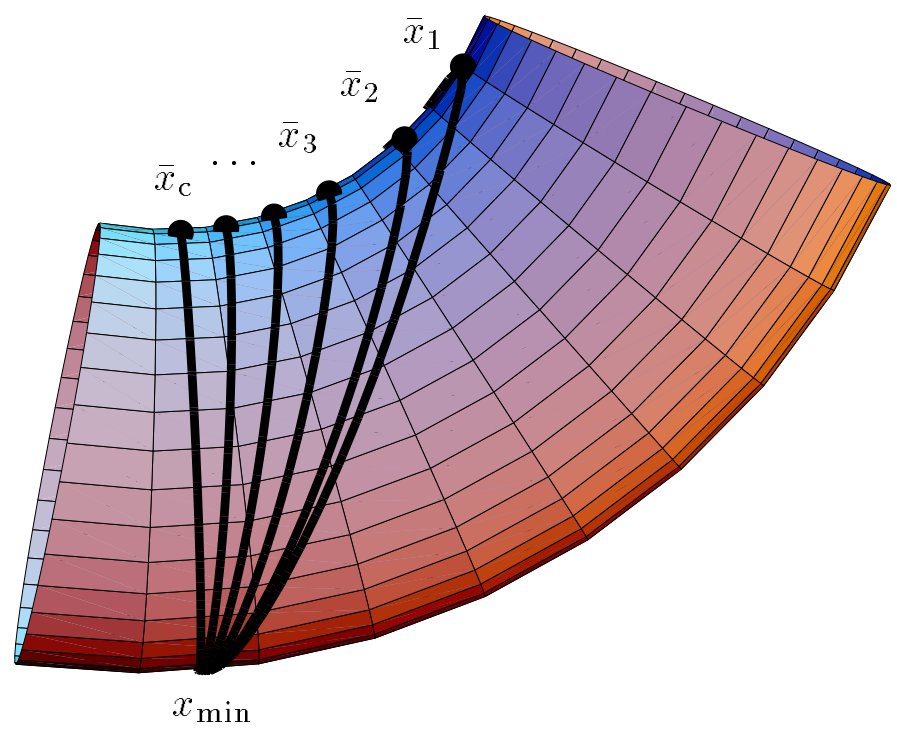

Fig. 17. Drawing of the successive paths passing through $x_{\min }$ and the local maxima $\bar{x}_{\mathrm{i}}$ for $i=1,2,3, \ldots$ of the curves which converge to the real minimax $\bar{x}_{\mathrm{c}}$.

where $\epsilon(t)$ is non-zero only around $\bar{x}_{\mathrm{c}}$. For $\epsilon(t)$ small enough, the deformed curve is still a non-contractible curve, however the maximum of the function along $\vec{x}_{\mathrm{d}}(t)$ is clearly less than the maximum at $\bar{x}_{\mathrm{c}}$ because the gradient $-\vec{\nabla} f$ points along the direction of decreasing $f$. This is a contradiction since $\bar{x}_{\mathrm{c}}$ is the minimum of $\{\bar{x}\}$. Hence $\vec{\nabla} f\left(\bar{x}_{\mathrm{c}}\right)=0$.

Morse theory goes on to give a set of inequalities relating the number of critical points to the changes in topology of the manifold. We refer the reader to the literature $[25,36,80,81,83,84$ for more details. We note that nothing depended critically on the fact that we were considering a non-contractible loop, it could well have been any non-contractible compact manifold, for example a sphere $S^{2}$. Hence non-trivial homotopy groups $\Pi_{\mathrm{m}}$ can imply the existence of non-minimal critical points. The crucial ingredient for the success of the minimax procedure was that the minimal critical point was non-degenerate. In fact, a function which only admits non-degenerate critical points is called a Morse function. To apply Morse theory however we do not truly require Morse functions. The function should simply be non-degenerate along every non-contractible loop.

The application of Morse theory to infinite dimensional manifolds was analyzed by Ljusternik-Snirelman [83]. A very readable account of the use of this theory was done by Taubes for the case of magnetic monopole [85]. The idea is to first establish the existence of non-contractible loops in the configuration space $M_{0}$, the space with net monopole number zero, i.e. $\Pi_{1}\left(M_{0}\right) \neq 0$. Secondly consider the configurations of an infinitely separated monopole-anti- 
monopole pair. This configuration is in $M_{0}$, and the non-contractible loop corresponds to rotating the monopole relative to the anti-monopole in isospace by one complete revolution. The minimal critical point corresponds to the situation where the monopole and anti-monopole have annihilated and all radiation has dissipated off to infinity leaving the quiescent (symmetry broken) vacuum behind. This critical point of zero energy is non-degenerate along every non-contractible loop. Taubes searched for the existence of a non-minimal critical point in the configurations bounded above in energy by the asymptotic critical point of the infinitely separated monopole-anti-monopole pair and bounded below by the energy (zero) of the vacuum. First it was shown that this infinite dimensional subset of the configuration space is in fact compact. With this information it is sufficient to exhibit a single non-contractible loop, with energy everywhere less than the energy of the infinitely separated pair, to be able to conclude that the minimax procedure will converge. It will of course converge to a different critical point than the trivial vacuum, since the vacuum is non-degenerate. The existence of this loop was shown [81] proving the existence of a non-minimal critical point.

Bagger et al 866 attempted to mimic this procedure for the Skyrme model. The configuration space for the Skyrme model corresponds to maps from $\mathbb{R}^{3}$ into the group $\mathrm{SU}(2)$ :

$$
\mathcal{C}:\left\{U(\vec{x}): \mathbb{R}^{3}+\infty \rightarrow \mathrm{SU}(2)\right\}
$$

$\mathbb{R}^{3}+\infty \equiv S^{3}$, hence $\mathcal{C}$ separates into disjoint sectors labeled by the homotopy classes $\Pi_{3}(\mathrm{SU}(2))=\mathbb{Z}$, the integer being of course the baryon number. Hence

$$
\mathcal{C}=\mathcal{C}_{0}+\mathcal{C}_{1}+\mathcal{C}_{2}+\cdots+\mathcal{C}_{-1}+\mathcal{C}_{-2}+\cdots
$$

The existence of non-contractible loops in $\mathcal{C}$

$$
\Pi_{1}(\mathcal{C}) \neq 0
$$

implies that

$$
\Pi_{1}\left(\mathcal{C}_{\mathrm{n}}\right) \neq 0
$$

and that they are actually all equal. Each sector actually contains each other sector, simply by constructing the requisite number of baryon-anti-baryon pairs, and moving, say, the anti-baryons as far away as is required.

$\Pi_{1}\left(\mathcal{C}_{0}\right)$ can be seen to be exactly the same as $\Pi_{4}(\mathrm{SU}(2))$. We can see this through the following construction. $\mathbb{R}^{3}+\infty$ can be thought of as the end 


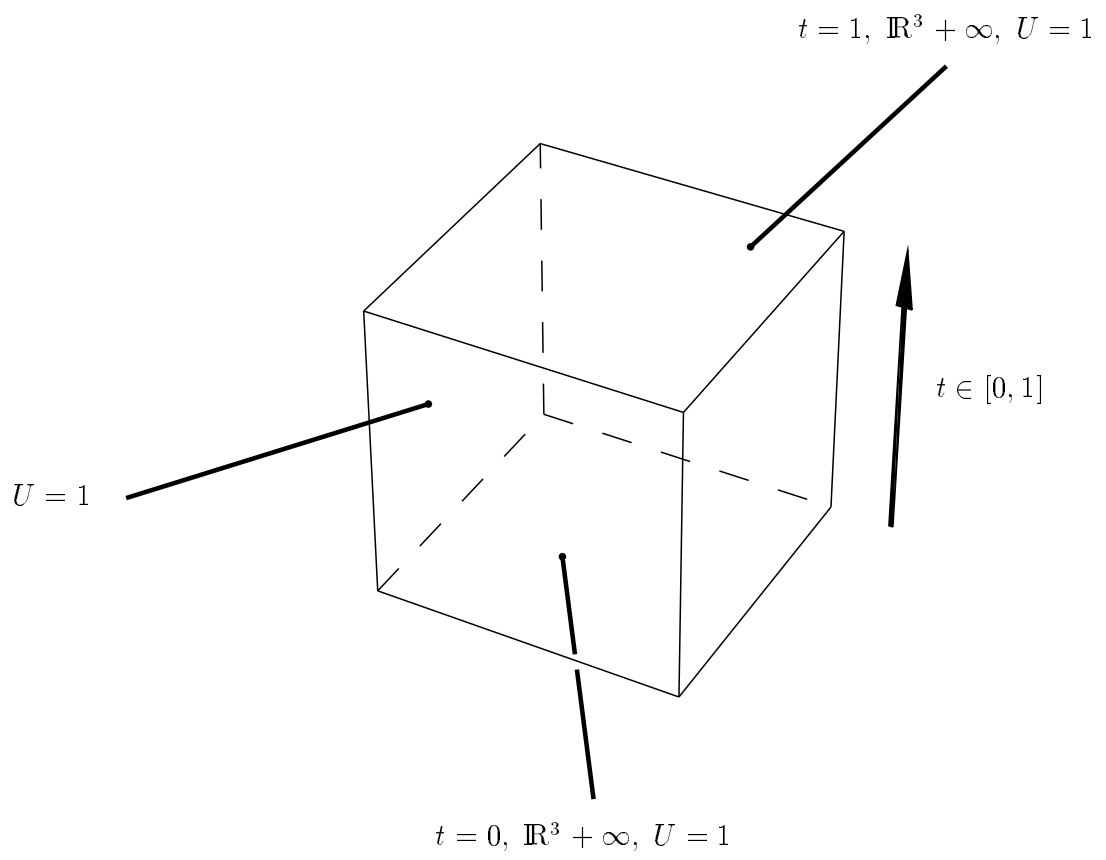

Fig. 18. Values of $U$ defined on the hypercube $\mathbb{R}^{4}$ of space.

cap of a 4-cube. The parameter along the fourth dimension corresponds to the parameter along the loop (see figure 18). If we start at $t=0$ with $U=1$, impose that $U=1$ along the vertical faces (because these faces also correspond to the point at infinity of $\mathbb{R}^{3}$ ), and $U=1$ at $t=1$, we define a loop in $\mathcal{C}_{0}$. However a 4 -cube with the surfaces identified is the same as $S^{4}, \mathbb{R}^{4}+\infty \equiv S^{4}$, hence we equally well define a mapping

$$
U(\vec{x}, t): S^{4} \rightarrow \mathrm{SU}(2)
$$

i.e. an element of $\Pi_{4}(\mathrm{SU}(2))$. It is known that 87

$$
\Pi_{4}(\mathrm{SU}(2)) \equiv \mathbb{Z}_{2}
$$

Hence there exist non-contractible loops in the space of configurations of the Skyrme model. These loops can most physically be realized as corresponding to a Skyrmion-anti-Skyrmion pair, as in the monopole situation, the two solitons are rotated relative to one another through one complete revolution. The difference between the Skyrme model and the monopole situation is that while loops involving further complete relative revolutions are distinct noncontractible loops for monopoles, for Skyrmions all loops with an odd number of complete revolutions are equivalent to each other and non-contractible while an even number of complete revolutions yields a contractible loop. Bagger et al 86] found, at least to first order in perturbation theory for large separation of the two solitons, that there were no non-contractible loops where the en- 
ergy was everywhere less than the energy of the asymptotic critical point of the infinitely separated Skyrmion-anti-Skyrmion pair. Augmenting the Skyrme model with an electromagnetic interaction, however, gave a sufficiently attractive Skyrmion-anti-Skyrmion potential for them to conclude the existence of a non-minimal critical point in this somewhat modified theory.

The problem was analyzed in greater detail by Isler et al [35], for the $B=0$ and $B=2$ situation together. Let $U_{\mathrm{S}}(\vec{x})$ be the field of a Skyrmion. Then for well separated Skyrmions or Skyrmion-anti-Skyrmion pair, the product ansatz suffices. Let

$$
\begin{aligned}
& U_{\mathrm{B}=2}(\vec{x})=R(t) U_{\mathrm{S}}\left(\vec{x}-\vec{x}_{1}\right) R^{\dagger}(t) U_{\mathrm{S}}\left(\vec{x}-\vec{x}_{2}\right), \\
& U_{\mathrm{B}=0}(\vec{x})=R(t) U_{\mathrm{S}}\left(\vec{x}-\vec{x}_{1}\right) R^{\dagger}(t) U_{\mathrm{S}}^{\dagger}\left(\vec{x}-\vec{x}_{2}\right)
\end{aligned}
$$

where $R(t)$ is an $\mathrm{SU}(2)$ matrix that introduces a relative iso-rotation. If $R(t)$ varies from any point in $\mathrm{SU}(2)$ to its antipodal point, i.e.

$$
\left.R(t)\right|_{t=1}=-\left.R(t)\right|_{t=0} \quad t \in[0,1]
$$

then $t$ parametrizes a non-contractible loop in $\mathcal{C}_{0}$ or $\mathcal{C}_{2}$. This is the usual topology which demonstrates $\mathrm{SU}(2)$ as the simply connected (double) cover of $\mathrm{SO}(3)$. It is a reasonably straightforward computation to find the energy to lowest order in the separation $d=\left\|\vec{x}_{1}-\vec{x}_{2}\right\|$,

$$
\begin{aligned}
& E_{\mathrm{B}=0}=2 E_{\mathrm{S}}-4 \pi f_{\pi}^{2} \kappa^{2} \frac{(1-\cos \theta)\left(3(\hat{n} \cdot \hat{d})^{2}-1\right)}{d^{3}} \\
& E_{\mathrm{B}=2}=2 E_{\mathrm{S}}+4 \pi f_{\pi}^{2} \kappa^{2} \frac{(1-\cos \theta)\left(3(\hat{n} \cdot \hat{d})^{2}-1\right)}{d^{3}}
\end{aligned}
$$

for

$$
R=e^{\mathrm{i} \theta \hat{\mathrm{n}} \cdot \vec{\tau} / 2}
$$

$E_{\mathrm{S}}$ is the energy of a Skyrmion and $\kappa$ is the coefficient of the $1 / r^{2}$ fall off in the Skyrmion profile function $f(r), f(r) \sim \kappa / r^{2}+O\left(1 / r^{6}\right)$ (see equation (13)). The potential $V$ serves to separate the (reduced) configuration space consisting of relative iso-rotations into two disjoint parts. Indeed this reduced space of configurations is isomorphic to a 3 -ball of radius $2 \pi$ modulo one identification. $\theta$ plays the role of the radius, $\hat{n}$ the unit vector giving the direction. Furthermore antipodal points are identified, $(\theta, \hat{n}) \equiv(2 \pi-\theta,-\hat{n})$. This identification is particularly reductive for $\theta=2 \pi$, the whole sphere at $\theta=2 \pi$ corresponds to $R=-1$ which is identified with the origin where $R=1$. 


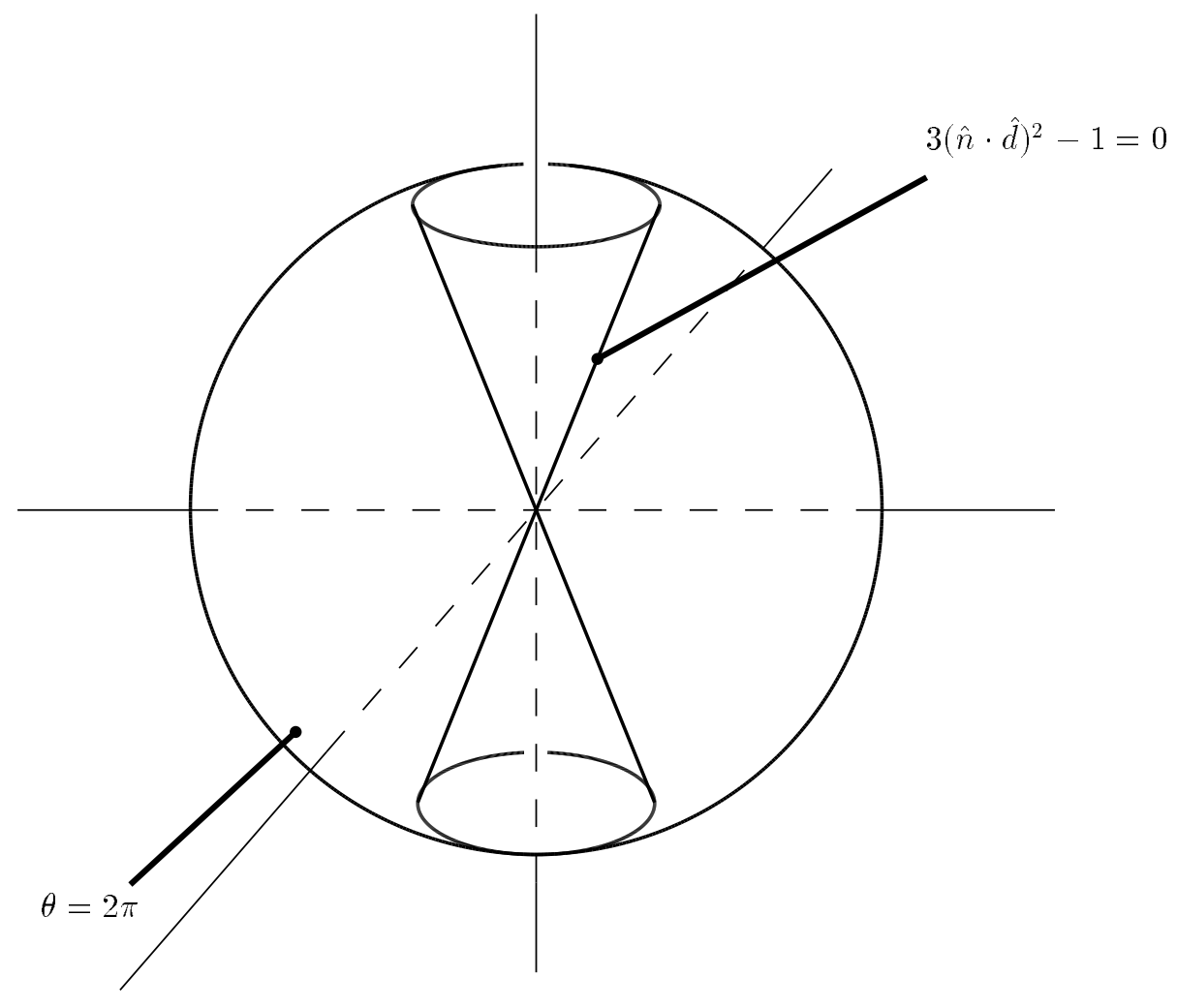

Fig. 19. Diagram of the attractive region for the Skyrmion-Skyrmion $B=2$ potential.

The factor $1-\cos \theta$ is positive semi-definite, and equal to zero only at $\theta=0$ or $2 \pi$. The function $3(\hat{n} \cdot \hat{d})^{2}-1$ varies from 2 when $\hat{n} \cdot \hat{d}=1$ to -1 when $\hat{n} \cdot \hat{d}=0$. Hence when the direction of the relative rotation is chosen such that $\hat{n} \cdot \hat{d}= \pm 1 / \sqrt{3}$, this defines a double cone that passes through the origin, demarcating the boundary between regions of relative attraction and relative repulsion (see figure 19). The regions of attraction have less energy than $2 E_{\mathrm{S}}$, while the regions of repulsion have more energy than $2 E_{\mathrm{S}}$.

If the attractive region is in the "time-like" region of these cones, which is the case for the case $B=0$, there is no curve which can pass from a point within this region to its antipode without either crossing over to the region of repulsion or touching the cone at its vertex. This is evident since a point and its antipode find themselves in opposite sides of the "forward" or "backward" light cone. All paths linking them must pass through the vertex or enter into the repulsive region which is not desired since the energy here is greater than that of two infinitely separated Skyrmions (or Skyrmion-anti-Skyrmion pair). Hence we cannot conclude the existence of a non-minimal critical point, we need to find a non-contractible curve where the energy is everywhere less than $2 E_{\mathrm{S}}$. Even though our curves are never greater than $2 E_{\mathrm{S}}$ (when they pass through the vertex) we cannot be assured that the minimax procedure will just 
converge to the asymptotic critical point (of energy $2 \epsilon_{\text {Skyrmion }}$ ). The situation just described is that which applies to the case $B=0$, i.e. a Skyrmion-antiSkyrmion pair. Here, there is no question that the minimum energy configuration is non-degenerate along every non-contractible curve. Hence it remains an open question whether indeed there are non-minimal critical points in the sector $B=0$.

Considering the case $B=2$ (see figure 19), the attractive and repulsive regions exchange with respect to $B=0$. Hence the attractive region is the "space-like" region relative to the cone. It is evident that now there exist non-contractible loops, which remain everywhere in the "space-like" region. They simply skirt around the cone to the other side of the origin where lies the antipode. Hence we show the existence of non-contractible loops which are everywhere lower in energy than $2 E_{\mathrm{S}}$. However this is still not enough to conclude the existence of non-minimal critical points. Indeed in the sector $B=2$ the energy functional fails to be a Morse function to a sufficient extent. The minimal critical point has been demonstrated to be a toroidal configuration with axial symmetry, in all but a rigorous, analytical mathematical proof. The axis of symmetry has no direction, the toroidal configuration rotated by $180^{\circ}$, about an axis orthogonal to the symmetry axis, is identical to the starting configuration. This implies that the minimal energy configuration is degenerate along a non-contractible loop. Hence it is the strongest possibility that the minimax procedure will converge simply to the minimum energy toroid.

Even though our exercise with Morse theory has led to no new solutions it is a worthwhile analysis allowing us to understand the model in a more profound way. There are several open questions raised by the analysis, two evident ones are do there exist sphalerons in the $B=0$ sector and do the existence of non-trivial higher homotopy groups imply existence of sphalerons. Indeed Morse theory has been used in relation with rational maps in a more recent article 34 .

\section{Dynamics of the Skyrme model: Soliton-Soliton scattering}

The dynamics of solitons is an extremely interesting and complicated problem. There are many different modes of excitation for a single soliton itself. There are certain modes, the zero modes of the classical small oscillation problem about the soliton, which properly belong to the soliton itself. They are usually treated semi-classically via Bohr-Sommerfeld type quantization rules. There are also regular vibrational and resonant modes which correspond to excited states of the soliton. Additionally there are modes which correspond to the scattering of (non-solitonic) waves off the soliton itself, for example, pions scattering from nucleons. 
Next one can consider the interaction of two solitons with each other. Here one can support many forms of reactions: scattering, deformation, bound states, annihilation among others. Soliton-anti-soliton annihilation is particularly problematic because of the coexistence of both perturbative and nonperturbative regimes. A physical example is evidently nucleon-anti-nucleon annihilation. The potential between a nucleon and an anti-nucleon has been obtained by Lu and Amado 13] using the product ansatz for large distances, and by Lu et al [14] for distances larger than $0.8 \mathrm{fm}$ using numerical methods. Physically there emerges a critical distance $d_{0}$ which is of the order of 0.8 $1.2 \mathrm{fm}$, outside of which the interaction between the particles is essentially repulsive. On the other hand if they attain this critical distance, they will quickly combine together into a lump of mesonic matter of baryon number zero. Numerical simulations of the classical system are essential to understand this complicated process [15]. It was shown that the reaction for transforming the pair of particles into a single lump of zero baryon number happens at the limit of causality and that the energy left by the disintegration remains localized for a relatively long time (until pion radiation waves disperses it into the vacuum) 16. The remaining part of the process, the emission of pion waves from the lump of mesonic matter, has been studied in the most detail, using path integral methods 17, or coherent state methods [18]. The results reproduce well the experimental phase shifts. We will not treat soliton-anti-soliton annihilation any further.

Even more complicated situations arise as we increase the soliton number. We can go to the point where one has an infinite number of solitons and all their various phases, fluid or solid, with possible crystalline structures. In this review we shall only discuss the low energy interaction of solitons, specifically Skyrmions, corresponding to the low energy scattering of nucleons.

Scattering of nucleons within the true microscopic theory, the standard model, is impossible to treat satisfactorily, essentially because of our inability to compute anything in the low energy domain. Even for very high energy scattering the final processes leading to hadronization are not computable from the microscopic theory. For low energy processes we have effective field theories, such as the Skyrme model, which afford more tractable descriptions of the physics involved. But even here, the baryons are represented as solitons and an exact quantum description of soliton states is still lacking. The only perturbative expansion feasible seems to be the semi-classical approximation.

The semi-classical approximation serves well to describe constituent properties of individual solitons. Essentially, the procedure is to identify the low energy, collective modes of the soliton and to quantize them. The interactions between solitons can only be treated perturbatively at large separations, by computing the effective interaction potential between them. 
In the past ten years there have been substantial advances in treating the part of the interaction at short distances and how to describe the scattering within the semi-classical approximation. Even the semi-classical approximation is not exactly solvable: soliton-soliton scattering involves an infinite number of degrees of freedom except for some very special cases of integrable models. The problem could be tractable if there were some way of truncating to a finite number of degrees of freedom. Exactly such a truncation was suggested by Manton 37.

In very general terms, one is interested in finding the low energy degrees of freedom of the two-soliton system. Typically one finds only a finite number of relevant degrees of freedom. The low-energy motion can then restrict itself self-consistently to these degrees of freedom.

The canonical example of such a situation was provided by the case of magnetic monopoles in the so-called BPS limit 38,58]. Here the inter-monopole force vanishes exactly, the magnetic Coulomb repulsion being exactly cancelled by an attractive force due to the existence of a massless scalar exchange. Hence there exist static solutions with two monopoles situated at arbitrary relative orientations and positions. This set of configurations corresponds to a submanifold of the set of all configurations and is called the moduli space. Indeed the characterization in terms of positions and orientations makes sense for monopoles when they are well separated, but as they come close together they lose their identity. What is preserved is the dimension of the space of moduli. For large separation, the moduli describe the position and orientation of each monopole: there are three degrees of freedom for the position of each monopole and one internal phase (related to the residual $\mathrm{U}(1)$ gauge symmetry) giving a total four degrees of freedom per monopole. As the two monopoles approach one another, only the dimension of the relative moduli space remains, the monopoles deform completely and fuse into a single entity. Clearly, since there are no forces, the moduli describe an equipotential surface. This surface is also the set of minimal energy configurations in the two monopole sector.

We can make an intuitive analogy with the surface of the earth. If this surface were perfectly spherical and frictionless, the potential as a function of radius would be (effectively) infinite at the radius of the earth and the equal to the usual gravitational potential for larger radii (figure 20). The radial motion, although not simple harmonic motion, corresponds to highly energetic modes. If we start at some point on this idealized earth, with an initial velocity that is tangent to the surface and arbitrarily small in amplitude, it is intuitively clear that the motion will remain very close to the surface of the earth. It is easy to prove in this case that the motion will follow geodesic curves on the surface of the earth (we will clarify somewhat the notion of geodesic later on).

The problem of the monopoles is analogous. If the initial conditions corre- 


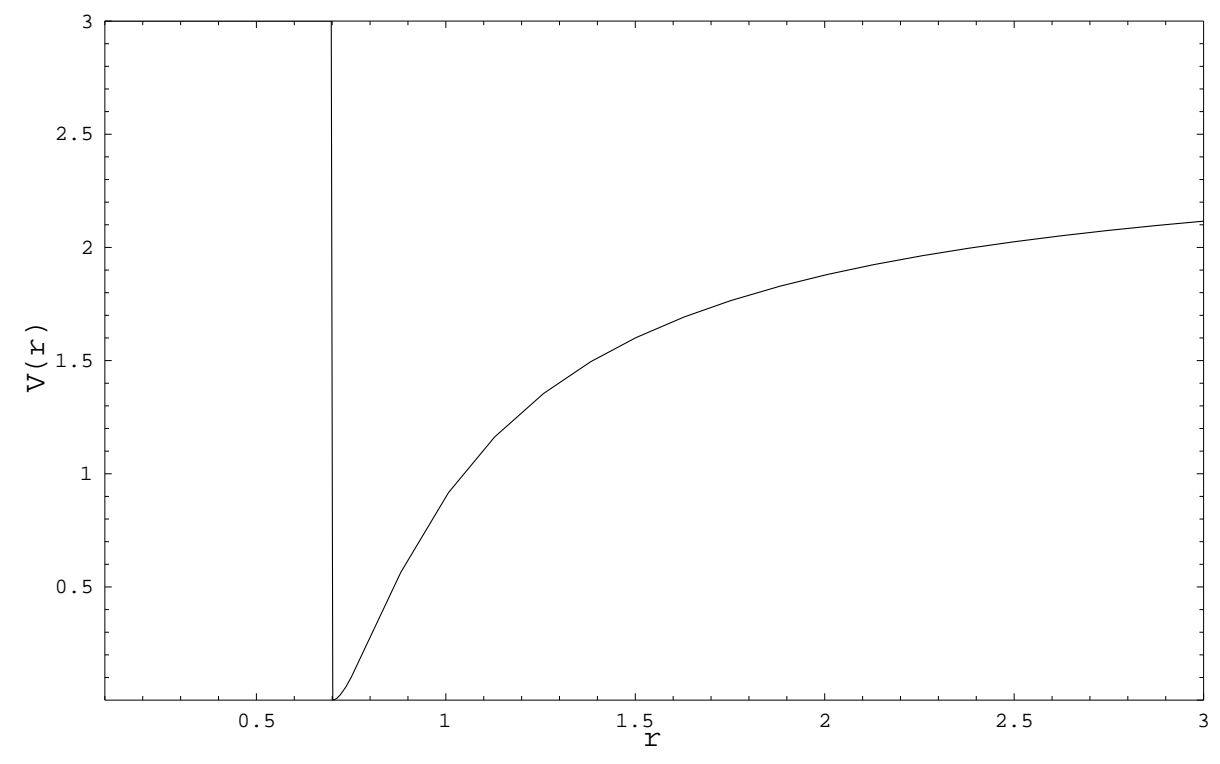

Fig. 20. Potential $V(r)$ on a hard, frictionless sphere as a function of the radius $r$. $R_{\mathrm{e}} \simeq 0.7$ is the radius of the sphere.

spond to being on a point of the moduli space of two monopole solutions, and the initial velocity is tangent to this surface and arbitrarily small, it was proposed [37, 38, 58 that the subsequent movement follows the appropriate geodesic in the manifold of the moduli space. This produces a concrete example of the truncation of the infinite number of original degrees of freedom to a finite number of relevant, effective degrees of freedom.

The general situation with solitons cannot be adequately described in terms of a moduli space as in the case of BPS monopoles. For example, Skyrmions or non-BPS limit (non-zero Higgs mass) monopoles experience inter-solitonic forces; hence no static solutions exist corresponding to arbitrary relative position of the solitons. Typically there do exist several low-lying critical points of the energy functional which should be involved and are important in the low-energy dynamics. In models where the solitons are not confined, one such critical point corresponds to infinitely-separated solitons. In addition, if configurations of energy lower than twice the energy of one soliton exist, then the minimum energy configuration represents another critical point corresponding to a bound state of two solitons. Furthermore there could be other metastable solutions such as the dibaryon of the Skyrme model 88, or the sphaleron solutions of the Weinberg-Salam model 36, 84, among others. The low-energy dynamics will restrict itself to these critical points and certain paths linking them together, as we will see in the next subsection. 


\subsection{General formalism}

Manton 37] suggested a possible truncation of the degrees of freedom to describe the low energy dynamics of the soliton in these more general situations. He suggested that the dynamics would truncate self-consistently to the union of all the low energy critical points and a set of curves which pass between these various critical points. These curves are alternatively taken to be the paths of steepest descent or the gradient flow curves linking the critical points together. The gradient flow method or steepest descent method gives rise to only slightly different sets of configurations if the critical points are truly low lying and the gradients are small, as we will now discuss. There is also another formalism called the valley method which also serves to give the paths which connect the critical points, however, we shall not discuss this method here 89 .

Gradient flow curves are mathematically described as the integral curves of the vector field corresponding to the gradient. This gives a first order differential equation:

$$
g_{\mathrm{ij}} \frac{\mathrm{d} x^{\mathrm{j}}(\lambda)}{\mathrm{d} \lambda}=-\frac{\partial V\left(x^{\mathrm{i}}(\lambda)\right)}{\partial x^{\mathrm{i}}}
$$

where $x^{\mathrm{i}}(\lambda)$ are the coordinates of a point along the curve. $g_{\mathrm{ij}}$ is the metric on the space of all configurations and $V\left(x^{\mathrm{i}}\right)$ is the potential defined on it. The initial directions taken corresponding to the unstable directions (negative modes) are extracted from the matrix of second derivatives of the potential at the position of the critical points.

The steepest descent curves, however, are defined in Manton 37] by the equation

$$
g_{\mathrm{ij}} \frac{\mathrm{d}^{2} x^{\mathrm{j}}}{\mathrm{d} \lambda^{2}}=-\frac{\partial V}{\partial x^{\mathrm{i}}}
$$

augmented with the boundary condition that

$$
\lim _{\lambda \rightarrow-\infty} x^{\mathrm{i}}(\lambda)=x_{0}^{\mathrm{i}}
$$

where $x_{0}^{\mathrm{i}}$ are the coordinates of the unstable critical point. These correspond, actually, to the approximate dynamical trajectories followed by the system starting at $t=-\infty$ at the critical point and moving in the unstable directions while neglecting terms that are quadratic in the velocities. $t$ corresponds 
exactly to $\lambda$. The exact dynamics would follow from the equation

$$
g_{\mathrm{mj}} \ddot{x}^{\mathrm{j}}+\left(\frac{\partial g_{\mathrm{ml}}}{\partial x^{\mathrm{k}}}-\frac{1}{2} \frac{\partial g_{\mathrm{kl}}}{\partial x^{\mathrm{m}}}\right) \dot{x}^{\mathrm{k}} \dot{x}^{\mathrm{l}}=-\frac{\partial V}{\partial x^{\mathrm{m}}}
$$

which is written in differential geometry as the geodesic equation

$$
g_{\mathrm{mj}}\left(\ddot{x}^{\mathrm{j}}+\Gamma_{\mathrm{kl}}^{\mathrm{j}} \dot{x}^{\mathrm{k}} \dot{x}^{\mathrm{l}}\right)=-\frac{\partial V}{\partial x^{\mathrm{m}}}
$$

where the Levi-Civita connection is

$$
\Gamma_{\mathrm{kl}}^{\mathrm{j}}=\frac{1}{2} g^{j m}\left(\frac{\partial g_{\mathrm{ml}}}{\partial x^{\mathrm{k}}}+\frac{\partial g_{\mathrm{mk}}}{\partial x^{\mathrm{l}}}-\frac{\partial g_{\mathrm{kl}}}{\partial x^{\mathrm{m}}}\right) .
$$

It is evident that the approximation is valid when $\dot{x}^{\mathrm{i}}$ are very small.

If we consider the same initial conditions with the dynamics augmented by a damping term representing friction, the steepest descent curves will naturally go over to the gradient flow curves

$$
g_{\mathrm{ij}} \frac{\mathrm{d}^{2} x^{\mathrm{i}}}{\mathrm{d} \lambda^{2}}+g_{\mathrm{ij}} \Gamma_{\mathrm{kl}}^{\mathrm{i}} \frac{\mathrm{d} x^{\mathrm{k}}}{\mathrm{d} \lambda} \frac{\mathrm{d} x^{\mathrm{l}}}{\mathrm{d} \lambda}=-\frac{\partial V}{\partial x^{\mathrm{i}}}-b g_{\mathrm{ij}} \frac{\mathrm{d} x^{\mathrm{i}}}{\mathrm{d} \lambda}
$$

( $b \gg 0$ measures the amount of damping in the system). Reparametrizing the curves with $\lambda \rightarrow b \lambda$ and taking $b \rightarrow+\infty$ we get

$$
0=\frac{\partial V}{\partial x^{\mathrm{i}}}+g_{\mathrm{ij}} \frac{\mathrm{d} x^{\mathrm{j}}}{\mathrm{d} \lambda}
$$

which is just the gradient flow equation.

We believe that all three equations yield essentially the same manifold to which the dynamics should truncate for a large variety of dynamics. What is required is that variations between the trajectories which determine the sub-manifolds go to zero. This will occur if the potential in the "transverse" directions is very steep. All three trajectories will be pushed together because of energy considerations: the trajectories cannot vary too far from the gradient flow trajectories because it costs too much in energy. In 4.2 .3 we will discuss a specific example wherein the three methods will be compared.

We will show with several examples how the truncation of the dynamics comes about and also some examples of how it can fail. 


\subsection{Examples of truncation for a few systems}

\subsubsection{Particle subject to a potential with $S^{2}$ symmetry}

First we treat the simplest, non-trivial example conceivable, which we have already mentioned: a particle on a spherical surface. Consider a Lagrangian in $\mathbb{R}^{3}$ with Cartesian coordinates $x^{\mathrm{i}}$ and Pythagorian metric $\delta_{\mathrm{ij}}$ :

$$
\begin{aligned}
L & =\frac{1}{2} \delta_{\mathrm{ij}} \dot{x}^{\mathrm{i}} \dot{x}^{\mathrm{j}}-\lambda\left(r^{2}-a^{2}\right)^{2} \\
& =\frac{1}{2}\left(\dot{r}^{2}+r^{2} \dot{\theta}^{2}+r^{2} \sin ^{2} \theta \dot{\phi}^{2}\right)-\lambda\left(r^{2}-a^{2}\right)^{2}
\end{aligned}
$$

where $r^{2}=\delta_{\mathrm{ij}} x^{\mathrm{i}} x^{\mathrm{j}}, \theta$ and $\phi$ are the usual spherical angular coordinates, $\lambda$ and $a^{2}$ are real and positive constants. For $\lambda=0$ the space of static solutions is just $\mathbb{R}^{3}$ and the general motion is along straight lines. For any non-zero $\lambda$ the set of static solutions is the set of sub-manifolds of $\mathbb{R}^{3}$ corresponding to critical points of the potential. For this potential the minimum corresponds to the manifold of the sphere of radius $a$. In addition there is an unstable critical point at the origin. The typical motion for any value of $\lambda$ depends on the total energy. The motion is of course bounded since the potential energy rises without bound for large $r$. The effective potential for the radial motion is

$$
\begin{aligned}
U(r) & =V(r)+\frac{l^{2}}{2 r^{2}} \\
& =\lambda\left(r^{2}-a^{2}\right)^{2}+\frac{l^{2}}{2 r^{2}}
\end{aligned}
$$

where $l$ is the (conserved) angular momentum. This potential has only one minimum, pushed further out from $r=a$ (its position for $l=0$ ) because of the angular momentum barrier. If the total energy of the system is fixed while $\lambda \rightarrow+\infty$ then the motion is energetically bounded to stay within a region that is arbitrarily close to the minimum of $V(r)$. This minimum corresponds to the surface $r=a$.

Indeed, the Lagrangian (263) after the substitution $r=a+\epsilon / \sqrt{\lambda}$ where $\epsilon=$ $\epsilon(t)$ and $a \gg \epsilon / \sqrt{\lambda}$, becomes

$$
\begin{aligned}
L & =\frac{1}{2} \frac{\dot{\epsilon}^{2}}{\lambda}-\lambda\left(\frac{2 a \epsilon}{\sqrt{\lambda}}+\frac{\epsilon^{2}}{\lambda}\right)^{2}+\frac{1}{2}\left(a+\frac{\epsilon}{\sqrt{\lambda}}\right)^{2}\left(\dot{\theta}^{2}+\sin ^{2} \theta \dot{\phi}^{2}\right) \\
& =\frac{1}{2}\left(a^{2}+\frac{\epsilon^{2}}{\lambda}+\frac{2 a \epsilon}{\sqrt{\lambda}}\right)\left(\dot{\theta}^{2}+\sin ^{2} \theta \dot{\phi}^{2}\right)+\frac{1}{2} \frac{\dot{\epsilon}^{2}}{\lambda}-\lambda\left(\frac{4 a^{2} \epsilon^{2}}{\lambda}+\frac{\epsilon^{4}}{\lambda^{2}}+\frac{4 a \epsilon^{3}}{\lambda \sqrt{\lambda}}\right)
\end{aligned}
$$




$$
\simeq \frac{a^{2}}{2}\left(\dot{\theta}^{2}+\sin ^{2} \theta \dot{\phi}^{2}\right)+\frac{1}{2} \frac{\dot{\epsilon}^{2}}{\lambda}-4 a^{2} \epsilon^{2}
$$

after applying the condition $a \gg \epsilon / \sqrt{\lambda}$. We see that the radial motion and the angular motion completely decouple as $\lambda \rightarrow+\infty$. To leading order the energy in the radial oscillations is

$$
E_{\mathrm{r}}=\frac{1}{2} 8 a^{2} \epsilon_{0}^{2}
$$

where $\epsilon_{0}$ is the amplitude of the solution $\epsilon(t)=\epsilon_{0} \sin (2 \sqrt{2} a \lambda t)$ to the equation of motion of $\epsilon$, and which should be taken to be independent of $\lambda . r$ then oscillates between $a-\epsilon_{0} / \sqrt{\lambda}$ and $a+\epsilon_{0} / \sqrt{\lambda}$, while the energy in the angular motion is

$$
E_{\theta, \phi}=\frac{1}{2} a^{2}\left(\dot{\theta}^{2}+\sin ^{2} \theta \dot{\phi}^{2}\right)
$$

The movement of the system with arbitrarily small initial velocity tangent to the sphere will be governed exclusively by the kinetic term of the Lagrangian. The dynamical constraint of being forced to live on the sphere of radius $a$ will be expressed by the appearance of the metric induced on the spherical sub-manifold of $\mathbb{R}^{3}$ by the Pythagorian metric already existing in the ambient space. This is obtained by choosing appropriate coordinates on the sub-manifold, $u^{1}=\theta, u^{2}=\phi$, the standard spherical coordinates, and to replace for $x^{\mathrm{i}}=x^{\mathrm{i}}\left(u^{\mathrm{j}}\right)$ in the metric of the ambient space

$$
\begin{aligned}
\mathrm{d} s^{2} & =\delta_{\mathrm{ij}} \mathrm{d} x^{\mathrm{i}} \mathrm{d} x^{\mathrm{j}} \\
& =\delta_{\mathrm{ij}} \frac{\partial x^{\mathrm{i}}}{\partial u^{\mathrm{k}}} \frac{\partial x^{\mathrm{j}}}{\partial u^{\mathrm{l}}} \mathrm{d} u^{\mathrm{k}} \mathrm{d} u^{\mathrm{l}} \\
& =a^{2} \mathrm{~d} \theta^{2}+a^{2} \sin ^{2} \theta \mathrm{d} \phi^{2} .
\end{aligned}
$$

So the effective Lagrangian will then be

$$
\begin{aligned}
L & =\frac{1}{2} g_{\mathrm{ij}} \frac{\mathrm{d} u^{\mathrm{i}}}{\mathrm{d} t} \frac{\mathrm{d} u^{\mathrm{j}}}{\mathrm{d} t} \\
& =\frac{1}{2}\left(a^{2} \dot{\theta}^{2}+a^{2} \sin ^{2} \theta \dot{\phi}^{2}\right) .
\end{aligned}
$$

The orbits of such a Lagrangian are great circles around the sphere, which are in fact exactly the geodesics of the metric (268), and are traced out by the motion at essentially constant velocity. We compare in figure 21 the "exact" numerical solution with the approximate solution $r=a$ and find a good agreement even for several revolutions around the sphere. 

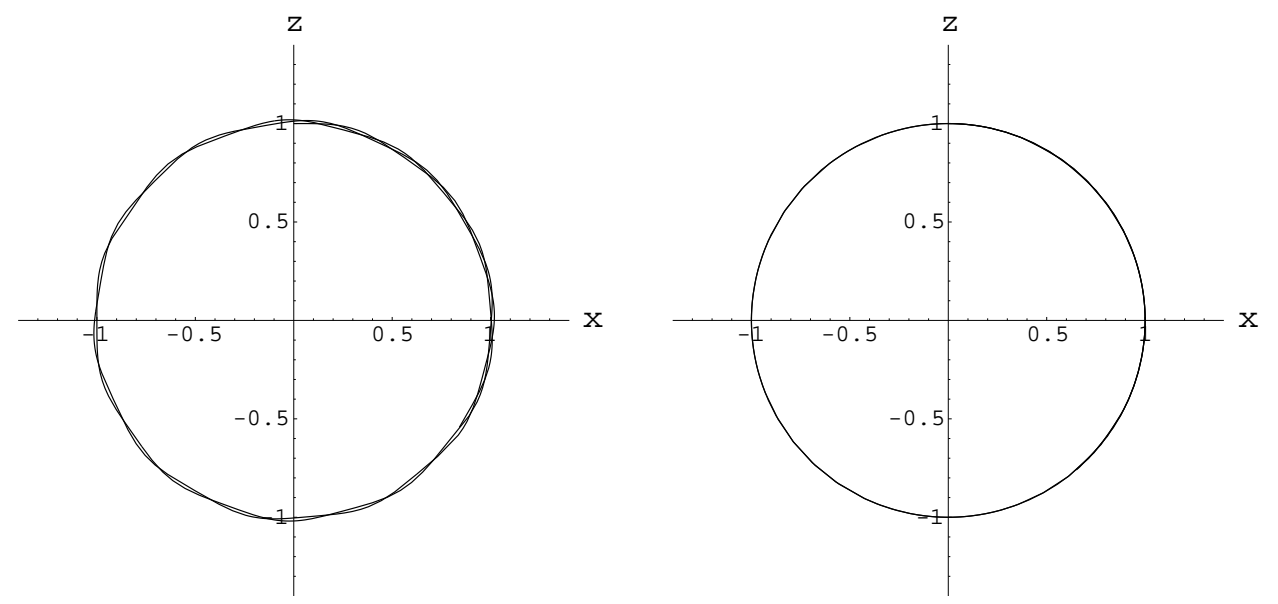

Fig. 21. Comparison of the "exact" (left) and approximate solution given by the path of steepest descent (right) for a particle subject to potential (263). They are both very close to the 2 -sphere $S^{2}\left(a=1, \lambda=3, r_{0}=a, \dot{r}_{0}=0\right.$ and $\dot{\theta}_{0}=0.5$ here).

\subsubsection{Particle on logarithmically deformed two-dimensional space}

A second instructive example is analogous to a scattering problem. This example shows how simple changes in the metric can induce radical changes in the motion.

Consider again the motion of a particle in $\mathbb{R}^{3}$ with Pythagorian metric and a potential corresponding to a Lagrangian

$$
\begin{aligned}
L & =\frac{1}{2} \delta_{\mathrm{ij}} \dot{x}^{\mathrm{i}} \dot{x}^{\mathrm{j}}-\lambda g^{2}\left(x^{\mathrm{i}}\right) \\
& =\frac{1}{2}\left[\dot{x}^{2}+\dot{y}^{2}+\dot{z}^{2}\right]-\lambda g^{2}(x, y, z) .
\end{aligned}
$$

We consider for the present purposes a function $g\left(x^{\mathrm{i}}\right)$ which admits solutions to the equation

$$
g\left(x^{\mathrm{i}}\right)=0 .
$$

Then as $\lambda \rightarrow+\infty$ the low energy motion will be restricted to the sub-manifold described by (271). Take the example

$$
g\left(x^{\mathrm{i}}\right)=z-\ln r=0, \quad \text { where } r=\sqrt{x^{2}+y^{2}} .
$$

The sub-manifold is the surface of revolution obtained by turning the function $z=\ln (x)$ about the $z$ axis (essentially it is a horn with a singular vertex at $x=y=0$ and $z=-\infty$ ). A good set of coordinates to use are the usual 
polar coordinates in the $(x y)$ plane. As $\lambda \rightarrow+\infty$ we can neglect the motion transverse to the surface, in which case $z=\ln r$ and the potential becomes zero while the kinetic term gives

$$
\begin{aligned}
L & =\frac{1}{2}\left(\dot{z}^{2}+\dot{r}^{2}+r^{2} \dot{\theta}^{2}\right) \\
& =\frac{1}{2}\left[\left(1+\frac{1}{r^{2}}\right) \dot{r}^{2}+r^{2} \dot{\theta}^{2}\right] \\
& \equiv \frac{1}{2}\left(g_{\mathrm{rr}} \dot{r}^{2}+g_{\theta \theta} \dot{\theta}^{2}\right) .
\end{aligned}
$$

Hence the dynamics corresponds to geodetic motion on the 2 dimensional manifold with metric

$$
\mathrm{d} s^{2}=\left(1+\frac{1}{r^{2}}\right) \mathrm{d} r^{2}+r^{2} \mathrm{~d} \theta^{2}
$$

It is clear that as $r \rightarrow+\infty$ the manifold becomes flat. The equations for the motion are

$$
\begin{aligned}
& \frac{\mathrm{d}}{\mathrm{d} t}\left[\left(1+\frac{1}{r^{2}}\right) \dot{r}^{2}\right]+\frac{\dot{r}^{2}}{r^{3}}-r \dot{\theta}^{2}=0 \\
& \frac{\mathrm{d}}{\mathrm{d} t}\left(r^{2} \dot{\theta}\right)=0 .
\end{aligned}
$$

This is just a "central force problem" with an $r$-dependent mass. The second equation corresponds to the conservation of angular momentum, $l$,

$$
\dot{\theta}=\frac{l}{r^{2}}
$$

Conservation of energy yields a second integral of motion

$$
\epsilon=\frac{1}{2}\left[\left(1+\frac{1}{r^{2}}\right) \dot{r}^{2}+\frac{l}{r^{2}}\right]
$$

The orbit equation is

$$
\begin{aligned}
\frac{\mathrm{d} r}{\mathrm{~d} \theta} & = \pm \frac{r^{2}}{l \sqrt{r^{2}+1}} \sqrt{2 \epsilon r^{2}-l^{2}} \\
& = \pm \frac{r^{2}}{\sqrt{r^{2}+1}} \sqrt{\frac{r^{2}}{b^{2}}-1}
\end{aligned}
$$




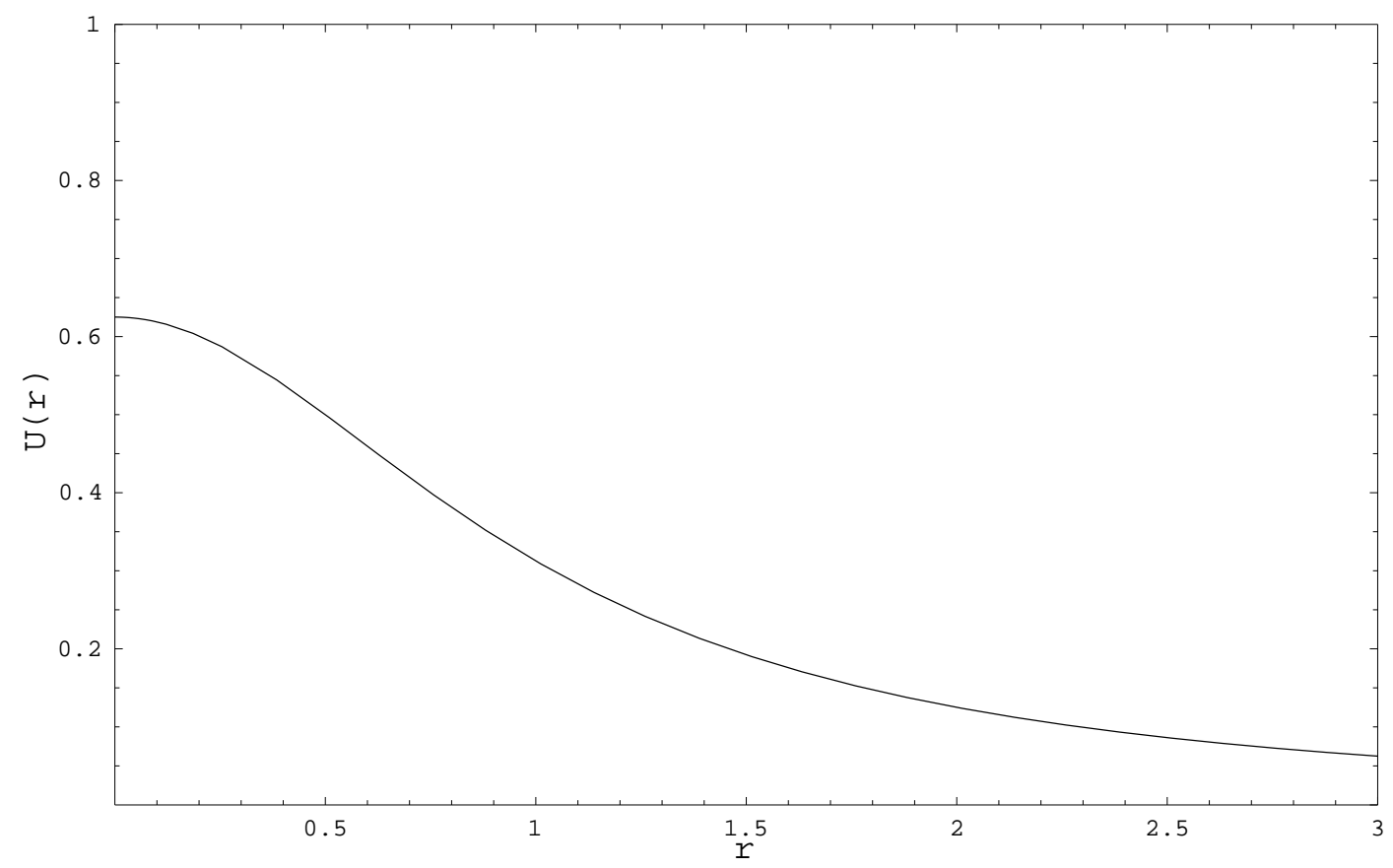

Fig. 22. Plot of the radial effective potential $U(r)$ for a particle restricted to move on the logarithmic surface $z=\ln r\left(v_{0}=1\right.$ and $\left.b=0.5\right)$.

where $\epsilon=v_{0}^{2} / 2, l=b v_{0}, v_{0}$ is the initial velocity and $b$ is actually the distance of closest approach. We see that $r \geq b$. We can define the effective potential 90] $U(r)=V(r)+l^{2} / 2 r^{2}$ by

$$
\frac{r^{2}}{l} \sqrt{2(\epsilon-U(r))}=\frac{r^{2}}{l \sqrt{r^{2}+1}} \sqrt{2 \epsilon r^{2}-l^{2}} .
$$

This yields

$$
U(r)=\frac{1}{r^{2}+1}\left(\epsilon+\frac{l^{2}}{2}\right)=\frac{v_{0}^{2}}{2} \frac{1+b^{2}}{1+r^{2}}
$$

which gives the graph in figure 22 .

There is no bound orbit; the turning point of the scattering occurs for $r=b$ when $U(b)=v_{0}^{2} / 2$, i.e. there is no more kinetic energy in the radial motion. The orbit equation is integral in terms of elliptic integrals and yields

$$
\theta(r)=\frac{b}{E(\mathrm{i} b, 1 / r)}
$$

where $E$ is the elliptic integral of the second kind. This gives a mild attractive potential. Typical geodesics bend slightly towards the singularity at $r=0$ for large impact parameter $b$, however, for $b$ near zero the particle makes several 
(a)

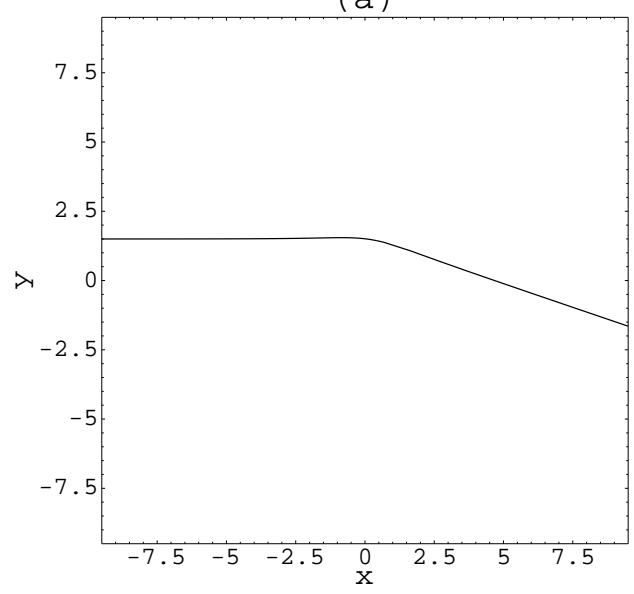

(c)

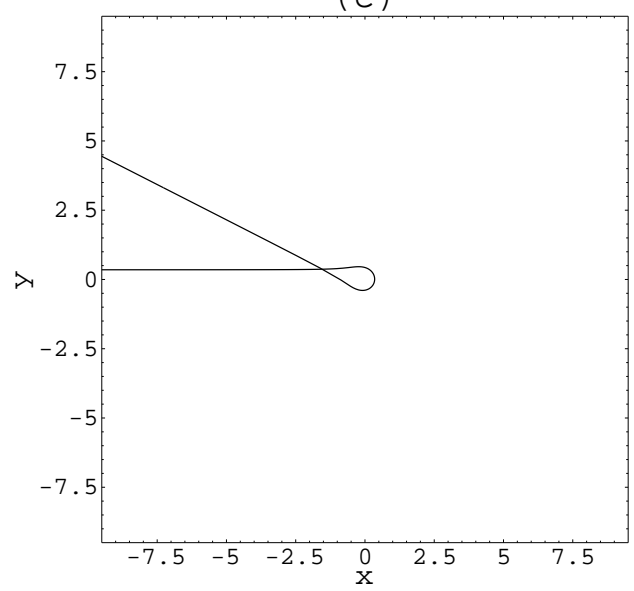

(b)

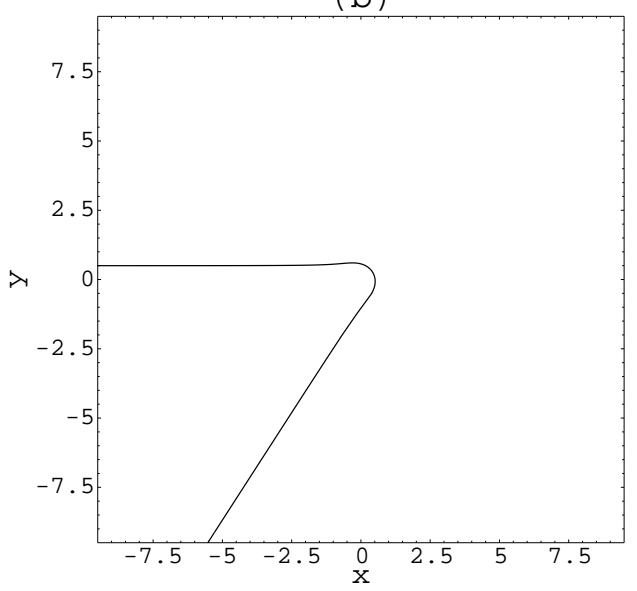

(d)

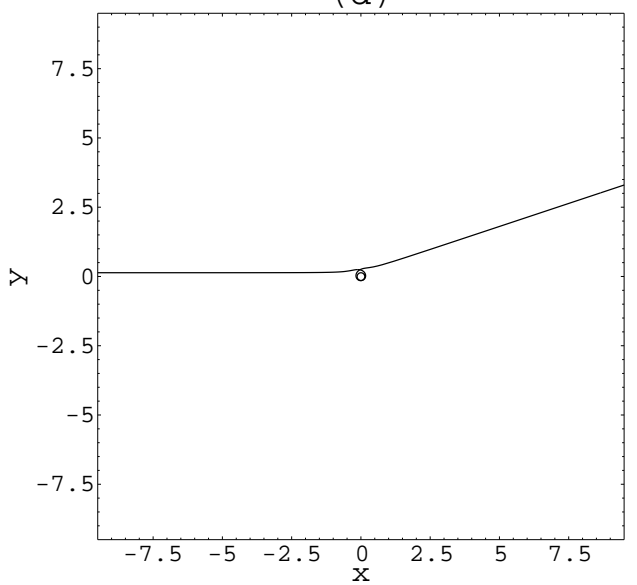

Fig. 23. Trajectories for the particle trapped on the logarithmic surface for different values of the impact parameter $b$ : (a) $b=1.5$, (b) $b=0.5$, (c) $b=0.35$ and (d) $b=0.135$ (The particle is coming from the left and its velocity is irrelevant).

revolutions about the singularity before returning to infinity (see figure 23). The $b=0$ geodesic is of course singular and the particle falls into the hole at $r=0$.

\subsubsection{Systems without a continuous set of static solutions}

Returning to the first example of the sphere, we augment it with a potential in the $z$ direction. Physically this could correspond to a uniform gravitational field. 
The Lagrangian that we consider is

$$
L=\frac{1}{2}\left(\dot{r}^{2}+r^{2}\left(\dot{\theta}^{2}+\sin ^{2} \theta \dot{\phi}^{2}\right)\right)-\lambda\left(r^{2}-a^{2}\right)^{2}-\Delta r \cos \theta .
$$

with $\Delta>0$ and $\Delta \ll \lambda$. Now the critical points of the potential correspond only to the absolute minimum located near the south pole of the sphere $(r=a$ and $\theta=\pi$ ), and a minimax near the north pole at $r=a$ and $\theta=0$. The conditions for the critical points are

$$
\begin{aligned}
& 4 \lambda\left(r^{2}-a^{2}\right) r-\Delta \cos \theta=0 \\
& \Delta r \sin \theta=0
\end{aligned}
$$

which give $\theta=0, \pi$ and

$$
r=a \pm \frac{\Delta}{8 \lambda a^{2}}+O\left(\frac{\Delta^{2}}{\lambda^{2}}\right)
$$

This case falls directly into the category for which Manton proposes that the motion truncates to the unstable manifold formed from the union of the curves of steepest descent or the gradient flow curves connecting critical points. The proposal corresponds to the following equations:

the gradient flow curve (255):

$$
\begin{aligned}
& \dot{r}=-4 \lambda r\left(r^{2}-a^{2}\right)-\Delta \cos \theta \\
& r^{2} \dot{\theta}=\Delta r \sin \theta \\
& r^{2} \sin ^{2} \theta \dot{\phi}=0
\end{aligned}
$$

or the paths of steepest descent 256

$$
\begin{aligned}
& \ddot{r}=-4 \lambda r\left(r^{2}-a^{2}\right)-\Delta \cos \theta \\
& r^{2} \ddot{\theta}=\Delta r \sin \theta \\
& r^{2} \sin ^{2} \theta \ddot{\phi}=0
\end{aligned}
$$

or the complete equations of motion (258)

$$
\begin{aligned}
& \ddot{r}=r\left(\dot{\theta}^{2}+\sin ^{2} \theta \dot{\phi}^{2}\right)-4 \lambda r\left(r^{2}-a^{2}\right)-\Delta \cos \theta \\
& 2 r \dot{r}+r^{2} \ddot{\theta}=\Delta r \sin \theta+r^{2} \sin \theta \cos \theta \dot{\phi}^{2} \\
& \frac{\mathrm{d}}{\mathrm{d} t}\left[r^{2} \sin \theta \dot{\phi}\right]=0 .
\end{aligned}
$$


As $\lambda / \Delta \rightarrow+\infty$, all three methods simply reduce to the sphere $r=a$, and the motion is constrained to this sphere with an effective potential

$$
V_{\text {eff }}(\theta)=a \Delta \cos \theta
$$

The difference in the three method gives rise to surfaces which vary by a thickness of the order of $\Delta / \lambda$. This can be important if this ratio is not small.

Numerical studies show that the motion is well approximated by motion on the truncated sub-manifold, with an important caveat: if the particle approaches the saddle point too closely the approximation can be misleading. To see this consider the case where the particle starts at the unstable critical point with a finite initial velocity. The movement when restricted to the unstable manifold will evidently correspond to revolutions about vertical great circles. Conservation of energy implies that the particle will always have enough energy to rise up to the saddle (point) and pass over the top. The actual motion however will necessarily excite the radial degree of freedom. If the dynamics conspire such that as the particle approaches the saddle point enough energy has been transferred to the radial degree of freedom to energetically prohibit the particle to pass over the saddle, the subsequent motion will fall back down the sphere on the same side in complete disaccord with the prediction of the truncated dynamics. We have actually numerically discovered this kind of deviation from the expected behaviour from the truncated dynamics (see figure 24). It is clear that the motion is very sensitive to the initial conditions and to the coupling between the low energy degrees of freedom and the high energy modes which govern the transfer of energy between these modes.

Another situation where the motion on the truncated manifold is very susceptible to small transverse oscillations occurs when the truncated set of configurations ceases to be a manifold. For example if the potential in (270) is of the form

$$
V\left(x^{\mathrm{i}}\right)=\lambda\left(g\left(x^{\mathrm{i}}\right) f\left(x^{\mathrm{i}}\right)\right)^{2},
$$

as $\lambda \rightarrow+\infty$ the solution of

$$
g\left(x^{\mathrm{i}}\right)=0
$$

and

$$
f\left(x^{\mathrm{i}}\right)=0
$$

will give in general two different sub-manifolds for the truncated dynamics. This is fine if the two manifolds are disjoint; then they will be separated by 

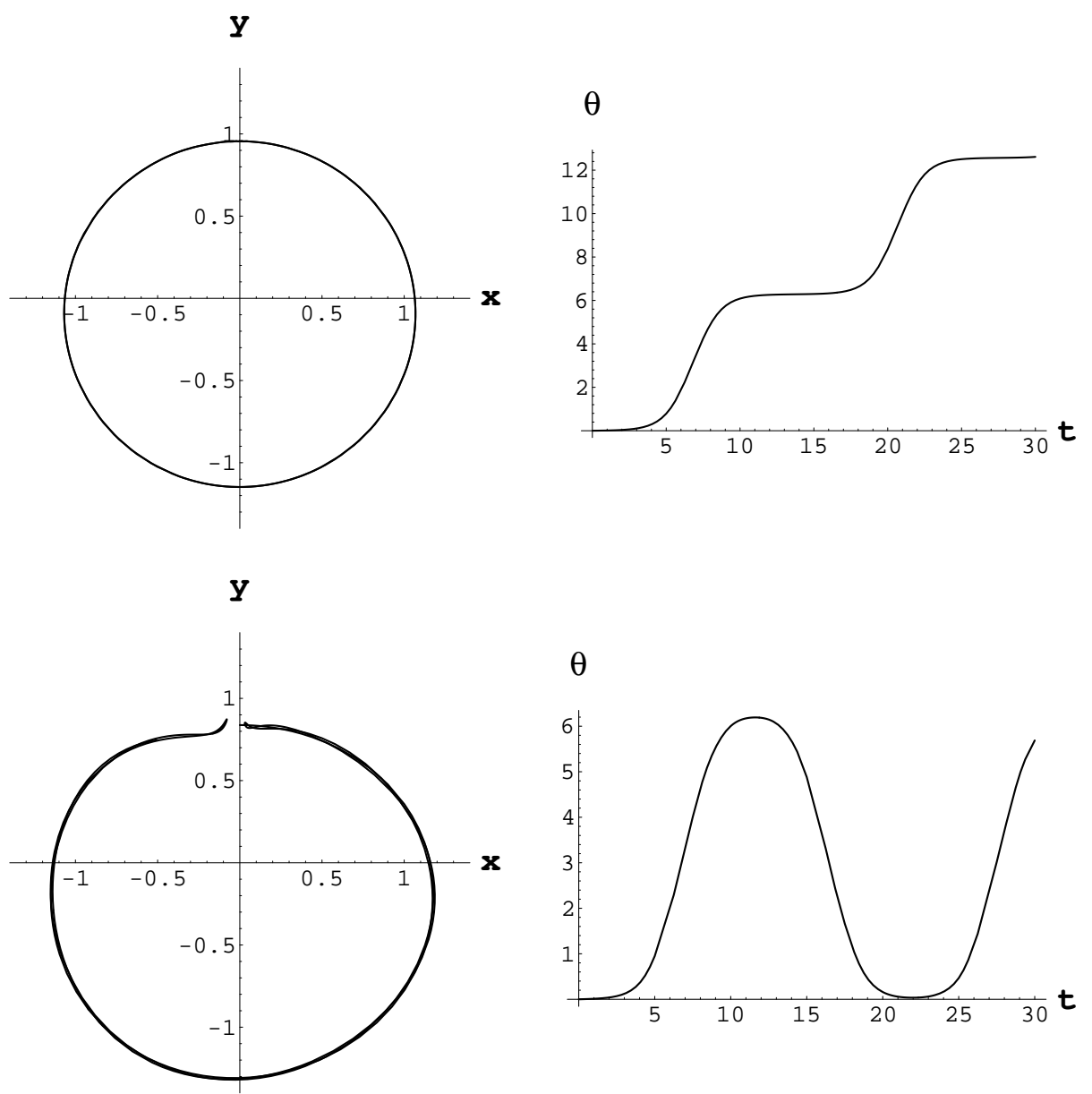

Fig. 24. Trajectories of the particle energetically constrained near a circle, and also subject to a linear, vertical gravitational potential (left) and a plot of the polar angle $\theta$ as a function of time $t$ during the process (right). For certain values of the parameters, the particle excites the radial mode sufficiently and does not quite make it over the top (even though it has more than enough energy to do so), in complete contradiction with the truncation method. Figures shown are obtained for $\lambda=3$ and $\delta=1$ (top), and for $\lambda=1$ and $\delta=1$ (bottom). 
large energy barriers and the dynamics will behave independently in each submanifold. However if they are tangent or even cross, the dynamics becomes very sensitive to transverse oscillations.

Take for example the two dimensional Lagrangian

$$
L=\frac{1}{2}\left(\dot{x}^{2}+\dot{y}^{2}\right)-\lambda\left[y\left(x^{2}+\left(y-\frac{1}{2}\right)^{2}-1\right)\right]^{2} .
$$

As $\lambda \rightarrow+\infty$ the low energy dynamics will truncate to the curves

$$
y=0
$$

or

$$
x^{2}+\left(y-\frac{1}{2}\right)^{2}=1
$$

These curves describe the $x$ axis and a circle of radius 1 which is just tangent to the $x$ axis at the origin (see figure 25). A low energy particle, in general, will effect tiny oscillations transverse to these curves and translate along these curves at roughly constant velocity. Suppose we start at a point on the circle with velocity tangent to the circle. Depending on the phase and amplitude of the radial oscillation that will necessarily be excited, at the moment that the particle passes the point of contact, the particle can easily be trapped by the valley along the $x$ axis and move on to $x \rightarrow+\infty$. Conversely, a particle moving along the $x$ axis, having a slight transverse oscillation, can be trapped in the circle. It is clear that the large scale low energy dynamics is not independent of the excitation of the high energy, modes however small they may be (see figure 26).

\subsection{Application of Manton's method to the $B=2$ sector of the Skyrme model}

We now apply Manton's formalism of gradient flow curves to the Skyrme model, for which the method was first put forward 37]. The first step is to single out the relevant critical points of the energy functional (in fact, the critical points of the potential energy of the model). Unlike the simple examples of the previous subsection, this task is quite hard, since a field theory is a system with an infinite number of degrees of freedom living (in this case) in a 3 dimensional space. Furthermore, non-linearity complicates the problem, and it is not surprising that coordinated numerical and analytical methods have to be used to find solutions to the equations of motion and to study their stability. We then describe how the global and relative degrees of freedom 

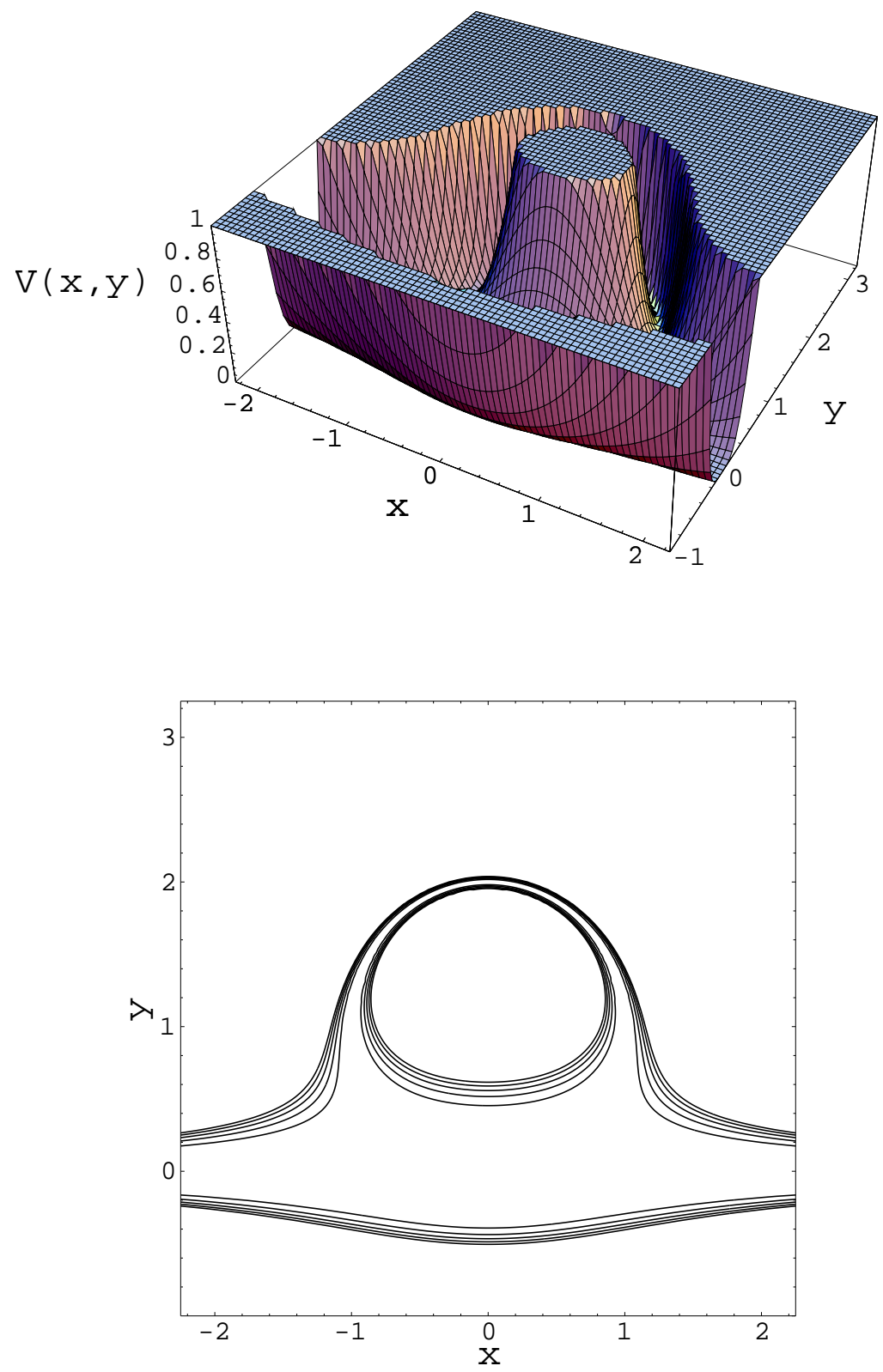

Fig. 25. Three-dimensional (top) and contour plot (bottom) of the potential of the Lagrangian (300) for $\lambda=20$ and $a=1$.

factorize. Finally we present the construction of the full unstable manifold linking together the critical points of this sector. 

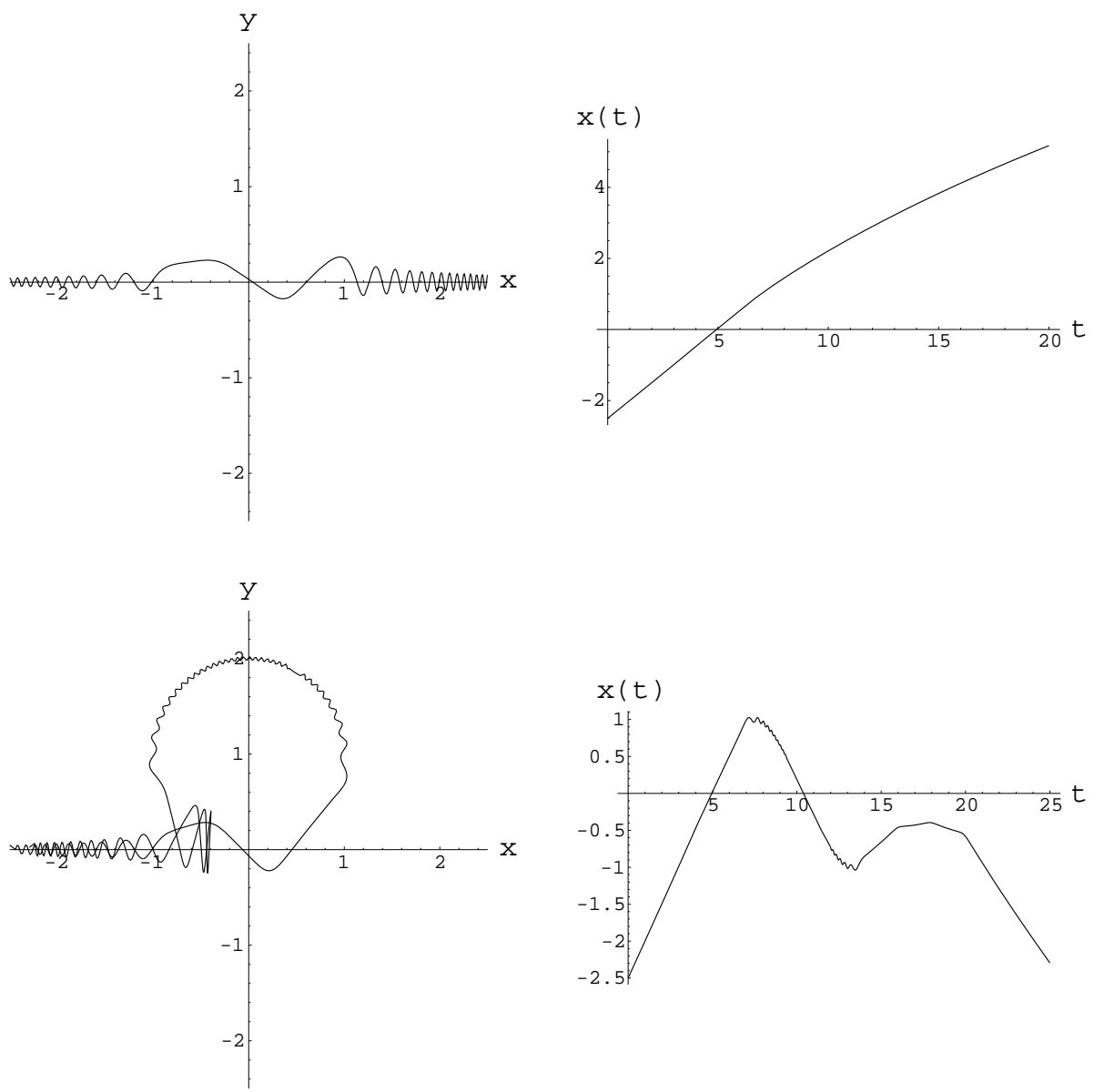

Fig. 26. Trajectories of a particle moving on a space made of a straightline with a circle tangent to (left) and a plot of its position along the $x$ axis as a function of time (right). The final state is extremely sensitive to initial conditions, with the particle either going through the intersection area at the first try (top) or getting caught in the loop and retracing its steps on the $x$ axis (bottom).

\subsubsection{Critical points of the $B=2$ sector}

The first solution of the Skyrme Lagrangian in the $B=2$ sector was already found by Skyrme [1] in the 60's. It is the product ansatz defined as follows:

$$
U_{\mathrm{PA}}(\vec{x})=A_{1}^{\dagger} U_{\mathrm{S}}\left(\vec{x}-\vec{R}_{1}\right) A_{1} A_{2}^{\dagger} U_{\mathrm{S}}\left(\vec{x}-\vec{R}_{2}\right) A_{2}
$$

where $U_{\mathrm{S}}$ is the Skyrmion field, $A_{1}$ and $A_{2}$ are $\mathrm{SU}(2)$ matrices defining the orientation of Skyrmion 1 and 2 respectively, and $\vec{R}_{1}$ and $\vec{R}_{2}$ represent their positions. The product ansatz is a solution of the equations of motion only in the limit where the distance between the particles $R \equiv\left\|\vec{R}_{1}-\vec{R}_{2}\right\|$ is infinite, since then, in the neighborhood of each Skyrmion, the other field is the identity and hence the product satisfies the equations of motion (and vice versa in the 
neighbourhood of the other Skyrmion). When $R$ is finite, the product ansatz is not a solution of the theory, but it still represents a good approximation to the configurations along gradient flow curves for large separations. This is true even though "Skyrme matter", like typical soliton fields, is quite soft and the mutual interactions of the fields deform the Skyrmions at a distance: they are no longer exact hedgehogs. However the ansatz is good enough to obtain an approximation to the potential between the solitons. This potential depends strongly on the relative orientation $A_{1}^{\dagger} A_{2}$ of the Skyrmions, and this allows it to be repulsive or attractive. Hence the solution at $R \rightarrow+\infty$ is a saddle point of the energy functional and necessarily a state of lower energy of most likely different structure exists. We will return to the potential and the product ansatz later since they are useful to understand the structure of the manifold of gradient flow curves of the system.

One expects to find in the $B=2$ sector a state which represents the deuteron, and indeed it would be another great triumph of the Skyrme model if such a state was found, with the right quantum numbers, binding energy, etc. The first localized state with baryon number two, which did not give the deuteron, is an immediate generalisation of the $B=1$ Skyrmion and was probably already contemplated by Skyrme himself in the 60's, although the result was first published by Jackson 88 in the 80 's. The $B=2$ hedgehog is defined (as we have seen in section 3.2.3) as

$$
U(\vec{x})=\mathrm{e}^{\mathrm{if}_{2}(\mathrm{r}) \cdot \vec{\tau}}
$$

where the profile function $f_{2}(r)$ has to be computed numerically like in the $B=1$ sector, but obeys the following modified boundary conditions: $f_{2}(0)=$ $2 \pi$ and $f_{2}(+\infty)=0$. This solution is a straightfoward generalization of the $B=1$ hedgehog, and we will call it the dibaryon in what follows to avoid any confusion with the ordinary hedgehog. The mass of the dibaryon happens to be about $1.855 \times 24 \pi^{2}$ in natural units, which translates roughly to 2.5 to 4.4 $\mathrm{GeV}$ (the value depends on the ones chosen for the parameters of the model $f_{\pi}$ and $e$ ) or about 3 times the mass of a single Skyrmion. This state can be shown to be unstable. It has much more energy than the product ansatz itself, and one expects the dibaryon to disintegrate at least into a pair of widely separated Skyrmions. We will see that exactly this has been observed in numerical simulations 91]. Hence the dibaryon is also a saddle point. Thus we have, so far, two types of saddle points from which to construct gradient flow curves. We are still missing, however, the minimum energy $B=2$ state to where these curves lead.

The state which is generally accepted as having the lowest energy in the $B=2$ sector was discovered independently by Verbaarschot [52] and Kopeliovich [54] using numerical methods, and actually proposed by Manton 53 by indirect methods related to dipole-dipole dynamics and symmetries. Its energy is about 


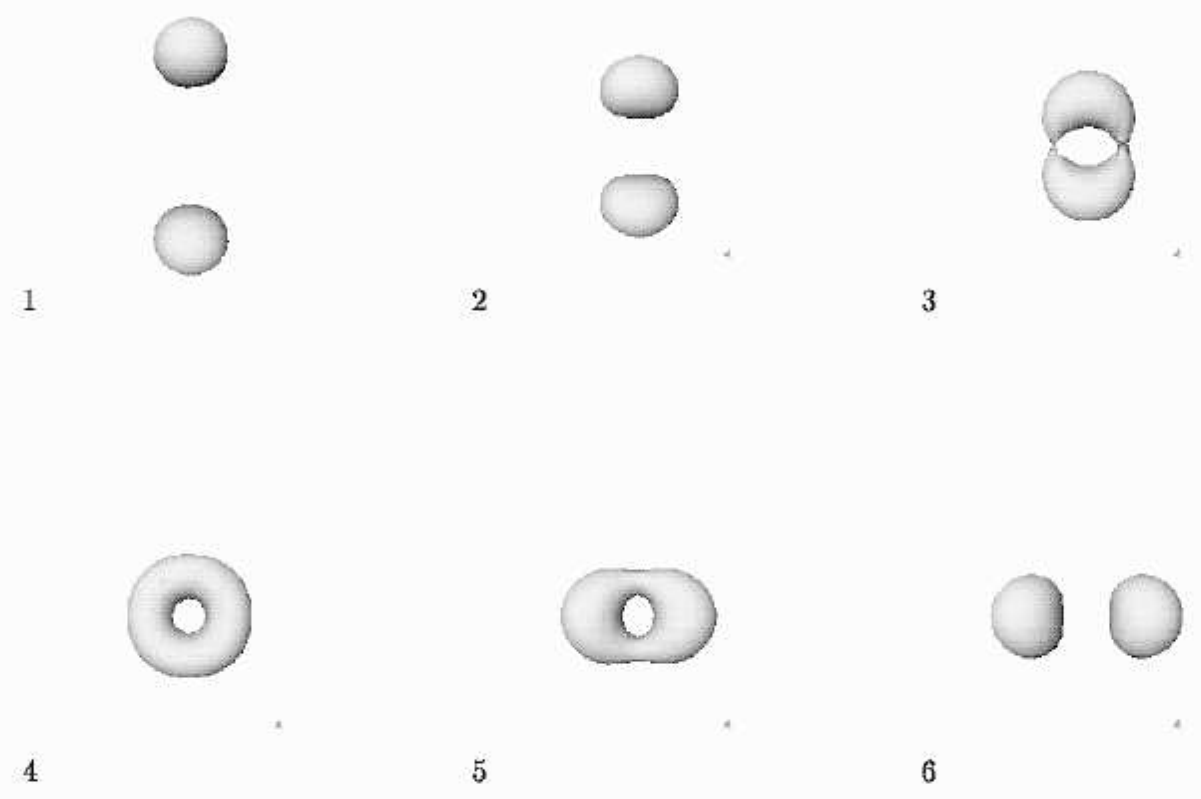

Fig. 27. Numerical simulation of the scattering of two Skyrmions via the $B=2$ torus.

$1.18 \times 24 \pi^{2}$ in natural units which is about $4 \%$ less than a pair of free Skyrmions. Quite intriguingly, the minimum energy state is toroidal. The deuteron, being the least bound of all nuclei, comprises of a proton and neutron that are quite spatially separated and distinct most of the time. The toroidal form well represents this spatial separation, however, it fails to reproduce the distinct character of the nucleons since the Skyrmions are completely deformed and have lost their separate identities. On the other hand, this state is expected to take an active part in the scattering processes of two Skyrmions, especially those with zero impact parameter and with the relative orientation which generates maximum attraction. In such a situation, the Skyrmions move toward each other, deform and come together in the toroidal state before dividing into two Skyrmions which depart along a direction perpendicular to the initial one (the scattering plane is fixed by the initial (iso)orientations of the Skyrmions) [53,92] (see figure 27 taken from reference [92]). This $90^{\circ}$ scattering is typical of head on collisions of identical solitons 93. The interpretation of this configuration as the deuteron is still controversial given its large binding energy (of the order of $100 \mathrm{MeV}$ instead of the $2 \mathrm{MeV}$ or so observed in nature), small radius and "strange shape" H Although it is not certain that

\footnotetext{
${ }^{3}$ One way to resolve this issue might be to compare the cross section for nucleonnucleon scattering computed from the Skyrme model with actual experimental measurements, and look for a signature predicted by the Skyrme model for Skyrmions
} 
this state is the absolute lowest energy state of the $B=2$ sector, it does seem reasonable that it is so. No other states with lower energy have been found so far, numerical simulations of Skyrmion-Skyrmion scattering have not uncovered another intermediate state 92], and numerous modes of oscillation around the toroid have been considered without finding any negative modes [62]. We will assume here that it is in fact the state with lowest energy and that the manifold of low energy dynamics of the $B=2$ sector consists of path joining the minimum and the two different families of saddle points.

To visualize the unstable manifold of the $B=2$ we need to know how the different configurations presented above are linked together by the dynamics. The number of degrees of freedom in the unstable and zero modes of each state will be most helpful. A solitonic system has an infinite number of degrees of freedom or modes that it can excite. Modes can be categorized according to their role in the stability of a particular configuration: negative modes generate paths followed by the system when it moves to another configuration of lower energy, positive modes are modes associated with oscillations around soliton configurations and zero modes correspond to rigid motions of the solitons arising from symmetries of the energy functional which are not respected by a given solution. If the system has low enough energy, only a finite number of these modes will be excited. In fact the system will first explore only the zero and negative modes (if there are any); higher energy excites the positive modes starting with those with the lowest frequencies. The parameters corresponding to zero modes are particularly important, and are called collective coordinates of the system. They form what is called the moduli space for the case where there are no negative modes. For example, in any BPS system, the collective coordinates corresponding to the zero modes are sufficient to describe the low energy motion of the system, which then corresponds to the geodesics on the moduli space. In cases where there is a weak potential acting between the solitons (like the Skyrme model), one has to also include the negative modes and the union of the corresponding gradient flow curves, to have enough freedom to let the system evolve in a satisfactory fashion. The number of degrees of freedom rises with the baryon number, since new collective coordinates enter to describe the relative motion of the particles, however the number can decrease if new symmetries arise via the dynamical evolution of the system.

going through the toroidal configuration. This is still an open problem since no general cross section for nucleon-nucleon scattering, neither classical nor quantum mechanical, has been computed so far (We present in the last subsections of this review our own semi-classical computations of nucleon-nucleon scattering angles with the Skyrme model under certain conditions.) 


\subsubsection{Factorization of global and relative coordinates}

Let $U(\vec{x})$ be a completely general configuration of the system obeying the conditions that $U(\vec{x})$ goes to the identity at infinity fast enough so that the total energy associated to the configuration is finite, localized in space, and the baryon number associated to the configuration is an integer. Such a configuration possesses generic collective coordinates corresponding to the invariance of the energy functional under the following 3 transformations:

$$
\begin{gathered}
\vec{x} \rightarrow \vec{x}+\vec{R}_{\mathrm{T}} \\
\vec{x} \rightarrow D(B) \cdot \vec{x} \\
U(\vec{x}) \rightarrow A U(\vec{x}) A^{\dagger} .
\end{gathered}
$$

In general a configuration is not invariant under these transformations, giving rise to nine zero modes in the fluctuation spectrum. $\vec{R}_{\mathrm{T}}$ is the position of the center of mass of the system, and is related to invariance of the energy under translation, the rotation matrix $D(B)$ associated to the $\mathrm{SU}(2)$ matrix $B$ parametrizes the rotational invariance of the system under global rotation, and finally the $\mathrm{SU}(2)$ matrix $A$ parametrizes the invariance of the system under global isospin rotation. Because of the absence of special symmetries of $U(\vec{x})$, the 9 parameters are completely independent, and if one gives them time dependence and quantizes the system treating them as dynamical variables, they give, respectively, the conserved total momentum $\vec{P}$, the conserved total angular momentum $\vec{L}$ and the conserved total isospin $\vec{T}$. The 9 global collective coordinates do not participate in the interesting dynamics of the system.

We will now apply these ideas to the $B=2$ sector of the Skyrme model in turn considering the product ansatz, the dibaryon and the torus. As we saw earlier, the product ansatz

$$
U_{\mathrm{PA}}(\vec{x})=A_{1} U_{\mathrm{S}}\left(\vec{x}-\vec{R}_{1}\right) A_{1}^{\dagger} A_{2} U_{\mathrm{S}}\left(\vec{x}-\vec{R}_{2}\right) A_{2}^{\dagger}
$$

is a solution of the theory only in the limit where $R=\left\|\vec{R}_{1}-\vec{R}_{2}\right\|$ is infinite; we will consider it to be arbitrarily large. The product ansatz is a solution described by 12 collective coordinates, or twice what is needed to describe a single Skyrmion: 3 parameters to define the position of the Skyrmion, and another 3 for its orientation or iso-orientation. This last fact comes from the spherical symmetry of the Skyrmion:

$$
\begin{aligned}
A U_{\mathrm{S}}(\vec{x}) A^{\dagger} & =\cos f(r)+\mathrm{i} \sin f(r) A \vec{\tau} A^{\dagger} \cdot \hat{x} \\
& =\cos f(r)+\mathrm{i} \sin f(r) \tau^{\mathrm{a}} D_{\mathrm{ab}}(A) \hat{x}^{b}
\end{aligned}
$$


hence

$$
A U_{\mathrm{S}}(\vec{x}) A^{\dagger}=U_{\mathrm{S}}(D(A) \cdot \vec{x})
$$

where $D(A)$ is the rotation matrix defined by (16) associated to the $\mathrm{SU}(2)$ matrix $A$. So iso-rotating the Skyrmion by a certain amount is exactly equivalent to rotating it in space by the same amount. Thus one requires only three parameters instead of six to describe the orientation (and iso-orientation). Another way to see this is to consider the following expression:

$$
U(\vec{x} ; A, B)=A U_{\mathrm{S}}\left(D(B)^{-1} \cdot \vec{x}\right) A^{\dagger}
$$

where the iso-orientation $A$ and the orientation $B$ are considered independent. This state is invariant under the following transformation of $A$ and $B$ :

$$
\begin{aligned}
& A \rightarrow A C \\
& B \rightarrow B C
\end{aligned}
$$

since

$$
\begin{aligned}
U(\vec{x} ; A C, B C) & =A C U_{\mathrm{S}}\left(D(B C)^{-1} \cdot \vec{x}\right) C^{\dagger} A^{\dagger} \\
& =A U_{\mathrm{S}}\left(D(B)^{-1} \cdot \vec{x}\right) A^{\dagger} \\
& =U(\vec{x} ; A, B)
\end{aligned}
$$

by equation (310). So $U(\vec{x} ; A, B)$ has a continuous redundancy parametrized by 3 angles, which arises from the spherical symmetry of the Skyrmion. The remaining 3 angles and 3 coordinates of the center of mass parametrize a manifold which can be written $S^{3} \times \mathbb{R}^{3}$. The product ansatz therefore has 6 independent parameters to describe each of the two particles, for a total of 12 .

We will now show how to re-express these 12 parameters $A_{1}, A_{2}, \vec{R}_{1}$ and $\vec{R}_{2}$ in terms of global and relative ones. To do this we will reproduce the discussion of Walhout and Wambach [20], who use the analogy with the treatment of a rigid body: the global coordinates will represent the transformation from the laboratory frame to the body fixed frame, and the relative coordinates will describe the system in the body fixed frame. Walhout and Wambach 20] chose the Skyrmions to be separated along the $z$ axis in the body fixed frame by a distance $R$, and the Skyrmion at the position $\vec{x}=-(R / 2) \hat{e}_{3}$ to be isorotated by $C$ relative to the other:

$$
\begin{aligned}
& A_{1}^{\mathrm{bf}}=1 \\
& A_{2}^{\mathrm{bf}}=C
\end{aligned}
$$




$$
\begin{aligned}
\vec{R}_{1} & =\frac{R}{2} \hat{e}_{3} \\
\vec{R}_{2} & =-\frac{R}{2} \hat{e}_{3} .
\end{aligned}
$$

In the lab frame, we will use $\vec{R}_{\mathrm{T}}$ to denote the position of the center of mass of the system, and we will position the Skyrmions in space using the inverse of the rotation matrix corresponding to the $\mathrm{SU}(2)$ matrix $B$, and a matrix $A$ will rotate the whole system in isospace. This gives us the product ansatz

$$
\begin{aligned}
& U_{\mathrm{PA}}^{\mathrm{lab}}(\vec{x})= A_{1} U_{\mathrm{S}}\left(\vec{x}-\vec{R}_{1}\right) A_{1}^{\dagger} A_{2} U_{\mathrm{S}}\left(\vec{x}-\vec{R}_{2}\right) A_{2}^{\dagger} \\
&=A U_{\mathrm{S}}\left[D(B) \cdot\left(\vec{x}-\vec{R}_{\mathrm{T}}-D(B)^{-1} \cdot \frac{R}{2} \hat{e}_{3}\right)\right] \times \\
& \quad C U_{\mathrm{S}}\left[D(B) \cdot\left(\vec{x}-\vec{R}_{\mathrm{T}}+D(B)^{-1} \cdot \frac{R}{2} \hat{e}_{3}\right)\right] C^{\dagger} A^{\dagger}
\end{aligned}
$$

with

$$
\begin{aligned}
A_{1} & =A B \\
A_{2} & =A C B \\
\vec{R}_{1} & =\vec{R}_{\mathrm{T}}+D(B)^{-1} \cdot \frac{R}{2} \hat{e}_{3} \\
\vec{R}_{2} & =\vec{R}_{\mathrm{T}}-D(B)^{-1} \cdot \frac{R}{2} \hat{e}_{3}
\end{aligned}
$$

which is clearly of the form:

$$
U(\vec{x})=A U\left(D(B) \cdot\left(\vec{x}-\vec{R}_{\mathrm{T}}\right)\right) A^{\dagger}
$$

where the global degrees of freedom are singled out. If we count the parameters we find that there are 13 of them instead of just the 12 which we started with. Walhout and Wambach 20] (inspired by some work by Verbaarschot 94]) show that one angle, which we shall define shortly, parametrizes a symmetry of the ansatz (319) and is therefore redundant. In the body fixed system, the product ansatz (319) obeys the following symmetry:

$$
U_{\mathrm{PA}}^{\mathrm{bf}}(\vec{x} ; C)=C(\mathrm{i} \vec{\tau} \cdot \hat{\mathrm{n}}) \mathrm{U}_{\mathrm{PA}}^{\mathrm{bf}}(\mathrm{D}(\mathrm{i} \vec{\tau} \cdot \hat{\mathrm{n}}) \cdot \overrightarrow{\mathrm{x}} ; \mathrm{C})(-\mathrm{i} \vec{\tau} \cdot \hat{\mathrm{n}}) \mathrm{C}^{\dagger}
$$

where $\hat{n}$ is any vector perpendicular to the $z$ axis, and $C$ is defined as follows:

$$
C(\vec{\tau} \cdot \hat{n})=(\vec{\tau} \cdot \hat{n}) C^{\dagger}
$$

If one writes $C$ as $e^{\mathrm{i} \vec{\gamma} \cdot \vec{\tau}}$, then this implies that $\hat{n}=\hat{\gamma} \times \hat{e}_{3}$. Let us check that 
(325) is indeed a symmetry of the product ansatz. Applying the transformation to the product ansatz in the body fixed frame one gets:

$$
\begin{aligned}
U_{\mathrm{PA}}^{\mathrm{bf}}(\vec{x} ; C) & =U_{\mathrm{S}}\left(\vec{x}-\frac{R}{2} \hat{e}_{3}\right) C U_{\mathrm{S}}\left(\vec{x}+\frac{R}{2} \hat{e}_{3}\right) C^{\dagger} \\
& \rightarrow C U_{\mathrm{S}}\left(\vec{x}+\frac{R}{2} \hat{e}_{3}\right) C^{\dagger} U_{\mathrm{S}}\left(\vec{x}-\frac{R}{2} \hat{e}_{3}\right)
\end{aligned}
$$

since $D(\mathrm{i} \vec{\tau} \cdot \hat{\mathrm{n}})$ is a rotation by $\pi$ around an axis perpendicular to the $z$ axis, and using (326). We then see that the Skyrmions change places, but in the limit where $R \rightarrow+\infty$, which is a necessary condition for the product ansatz to be a solution of the equations of motion, the order of the Skyrmion matrices is irrelevant since the two commute. Then (325) is really a symmetry of the solution within the product ansatz. It is also a symmetry of the Lagrangian (since it only consists of global rotation and isorotation) and of the subsequent evolution. The symmetry (325) is valid for any vector $\hat{n}$ as long as it is perpendicular to the $z$ axis: the angle which defines the orientation of $\hat{n}$ in the $(x y)$ plane actually spans a whole family of discrete symmetries of the ansatz. Since $\hat{n}=\hat{\gamma} \times \hat{e}_{3}$, rotating $\hat{n}$ in the $(x y)$ plane by the angle $\theta$ also rotates $\hat{\gamma}$ by the same amount in the same plane, which in turn is equivalent to acting on the matrix $C$ as follows:

$$
C \rightarrow e^{\mathrm{i} \frac{\theta}{2} \tau^{3}} C e^{-\mathrm{i} \frac{\theta}{2} \tau^{3}}
$$

It is easily checked that for the product ansatz this symmetry takes the following form:

$$
U_{\mathrm{AP}}^{\mathrm{bf}}(\vec{x} ; C)=e^{-\mathrm{i} \frac{\theta}{2} \tau^{3}} U_{\mathrm{AP}}^{\mathrm{bf}}\left(D\left(e^{\mathrm{i} \frac{\theta}{2} \tau^{3}}\right) \cdot \vec{x} ; e^{\mathrm{i} \frac{\theta}{2} \tau^{3}} C e^{-\mathrm{i} \frac{\theta}{2} \tau^{3}}\right) e^{\mathrm{i} \frac{\theta}{2} \tau^{3}}
$$

or that, in the laboratory reference frame, the product ansatz is invariant under the combined transformations:

$$
\begin{aligned}
& A \rightarrow A e^{-\mathrm{i} \frac{\theta}{2} \tau^{3}} \\
& B \rightarrow e^{\mathrm{i} \frac{\theta}{2} \tau^{3}} B \\
& C \rightarrow e^{\mathrm{i} \frac{\theta}{2} \tau^{3}} C e^{-\mathrm{i} \frac{\theta}{2} \tau^{3}} .
\end{aligned}
$$

This shows that the angle of rotation $\theta$ around the $z$ axis implicitly contained in $C$ is redundant and does not play a role in the describing the configuration. $C$ is then only parametrized by 2 angles: one for the orientation of $\hat{\gamma}$ in the (xz) plane, and the magnitude $|\vec{\gamma}|=\gamma$, by which the second Skyrmion is rotated relative to the other. We then have, as we should, 9 global collective coordinates $\left(A, B\right.$ and $\left.\vec{R}_{\mathrm{T}}\right)$ and 3 relative ones $(C$ and $R$ ) giving a total of 12 instead of 13 . One should carefully note that equation (329) by no 
means implies that the product ansatz possesses a continuous symmetry: it only does if one writes it using too many parameters as collective coordinates (exactly like the case for the ordinary Skyrmion described above by equation (314)). Once the correct number of collective coordinates has been used, the only symmetries that the product ansatz possesses are 3 reflection symmetries relative to the $(x y),(y z)$ and $(x z)$ planes (all those discrete symmetries are exact only in the limit where $R \rightarrow+\infty)$.

\subsubsection{The construction of the unstable manifold}

Let us suppose that the Skyrmions always keep the same hedgehog shape no matter how strongly they interact with each other. Then one could obtain the Lagrangian of the system by giving time dependence to the collective coordinates, and replacing the product ansatz in the Skyrme Lagrangian and computing the requisite integration over all space. These 12 degrees of freedom then describe a manifold, which we shall note $M_{12}$, with a metric and a potential induced by the kinetic and potential parts of the Lagrangian, respectively [95]. The Skyrmion-Skyrmion or nucleon-nucleon dynamics is then obtained by doing classical or quantum mechanics in the curved space of the manifold $M_{12}$. Unfortunately, this program is not correct since the Skyrmions deform and do not stay in the form of a hedgehog when they interact (obviously since they deform enough to merge into a torus), and $M_{12}$ has no hope of describing the full dynamics in the $B=2$ sector. But one expects that the unstable manifold (i.e. that obtained by gradient flow curves) of the $B=2$ sector, which we shall call $\mathcal{M}_{12}$, and $M_{12}$ will become approximately equal for $R$ sufficiently large.

Numerical studies have shown that the product ansatz give a surprisingly good approximation to $\mathcal{M}_{12}$ up to a separation of just a few fm (depending on the initial relative orientations of the Skyrmions). This enables one to extract useful information on the Skyrmion-Skyrmion dynamics relatively easily. One can for instance compute an approximation to the Skyrmion-Skyrmion potential even for moderate values of the separation $R$. The separation is not a zero mode of the energy any longer, but it is a relatively "soft" mode such that the potential energy does not vary by large values as a function of $R$, as long as $R$ is large. In low energy scattering, the $R$ mode can be considered a "slow" mode compared to the angular variables representing the rotation of particles which are "fast" variables. We will describe exactly such an approach in the next subsection.

The first calculation of the Skyrmion-Skyrmion potential was obtained by Skyrme himself and is defined by:

$$
V_{\mathrm{SS}}=E_{\mathrm{B}=2}-2 M_{\mathrm{S}}
$$


where $E_{\mathrm{B}=2}$ is the static energy of the system. This involves integrating Skyrmion profiles over all space, which is best done using an expansion in inverse powers of the separation $R$. To leading order one finds

$$
V_{\mathrm{SS}}(R, C)=-4 \pi f_{\pi}^{2} \kappa^{2} \frac{D(C)_{\mathrm{ii}}-3 \hat{R}^{\mathrm{i}} D(C)_{\mathrm{ij}} \hat{R}^{\mathrm{j}}}{R^{3}}
$$

which only depends on the relative coordinates: 2 angles and the distance $R$.

Subsequently, there has been a great deal of work on the extraction of the nucleon-nucleon potential from the Skyrme model and its comparison with traditional nuclear potentials [9,10,20,55]. Early computations, mainly using the product ansatz, quickly showed that the general tensorial form of the potential so obtained was in good agreement with what is known of the nuclear force (1-2 pion exchange, repulsive core, etc.). However, a particularly disturbing defect haunted the problem for many years: the absence of a central attraction at medium range. This was all the more disturbing since it is precisely this part of the nuclear force which binds nucleons together into nuclei. Many ansätze were tried generalizing the product ansatz: the modified product ansatz [9] (the Skyrmions were given the freedom to change their radii as they interacted, but with little gain in the central attraction), the symmetrized product ansatz 96] (a step in the right direction since it was symmetric under the exchange of the particles, and provided some central attraction but at the expense of the long range behaviour), and more recently the instanton method 55] (which combines the advantages of all the previous ansätze), but the crucial central attraction still eluded all efforts.

It was in two 56, 97 exact, numerical analyses that a central attraction was found. The two approaches bifurcated in the way that they included physical, quantum nucleonic states. In the approach of Verbaarschot et al [56] the potential is computed using numerical relaxation methods on a $20 \times 20 \times 40$ regular lattice for fixed separation and imposing only the discrete symmetries of the most attractive channel ( see below). The separation was defined to be the distance between the respective centers of baryon number density. They found a reasonable attraction (roughly $70 \mathrm{MeV}$ ) in the central channel. In their approach, the physical nucleonic states where incorporated by treating the angular and iso-angular coordinates as rigid quantum rotors, and the radial motion was treated semi-classically using the WKB method. They found that the kinetic energy of the angular and iso-angular motion was sufficient to destabilize any possible bound state. However the numerical analysis was recently repeated using a more refined method 98, the finite element approach, which permits an irregular lattice. In this way, for the same amount of computing time, the algorithm can sample the critical regions with a finer grid, and consequently improve the accuracy. This work shows that indeed the central channel is sufficiently attractive to support a bound state, the deuteron, 
within the WKB method for the radial degree of freedom. On the other hand in the work of Walhout and Wambach 97], although the potential was again computed for static Skyrmionic configurations, the nucleon-nucleon potential was extracted by projection onto asymptotic quantum nucleon states à la Jackson, Jackson and Pasquier 9]. Here also a central attraction was found, however the further analysis necessary to establish a bound state was not presented. Walet and Amado 99] further improved the results by including the $\Delta$ resonance as intermediate state, as well as some gluonic corrections. With all these corrections, the thus obtained Nucleon-Nucleon potential is indeed quite close to traditional (phenomenological) nuclear potentials, even though the arbitrariness in the definition of the distance between a pair of particles when they are very deformed (at small $R$ ) makes it hard to judge. Here we will only be interested in the long range part of the potential which takes the form of the one-pion exchange potential.

The tensorial form of the potential reveals how Skyrmions will react to their relative orientations. The different situations can be catalogued in three cases or channels. In the hedgehog-hedgehog channel (HH), $C=1$ and the potential is zero (no one-pion exchange potential). As $R$ gets smaller then other interactions take over and build a repulsive core. In this channel, the Skyrmions stay roughly hedgehog-like until $R$ is of the order of $1 \mathrm{fm}$. If the second Skyrmion is rotated by $\pi$ around the axis of separation, $C=\mathrm{i} \tau^{3}$, then a strong repulsive interaction keeps the Skyrmions apart and the potential energy rises quickly as $R$ decreases. In this channel (REP), Skyrmions only stay roughly spherically symmetric for $R$ greater than about $1.5 \mathrm{fm}$. The most interesting case is the so-called most attractive channel (MAC), where one Skyrmion is rotated relative to the other by $\pi$ around a direction perpendicular to the axis of separation. In this channel, the Skyrmions always attract and come closer and closer together until they fuse into the toroidal configuration. This is the most studied and phenomenologically interesting case since it is believed to contain the nucleon-nucleon binding dynamics. Of course, in a realistic general collision of nucleons, one does not stay in any one of these channels (spins are generally not correlated during real scattering) and situations should be hybrids of the ones described so far.

By freezing the relative iso-orientations, the two collective coordinates represented by $C$ disappear, and the configurations then span a 10-dimensional manifold: $\mathcal{M}_{10}^{\mathrm{HH}}, \mathcal{M}_{10}^{\mathrm{REP}}$ and $\mathcal{M}_{10}^{\mathrm{MAC}} \cdot \mathcal{M}_{10}^{\mathrm{HH}}$ and $\mathcal{M}_{10}^{\mathrm{REP}}$ are actually not very interesting since they would not represent very accurately the dynamics of the system. This is because the HH and REP channels lead to relatively energetic states: the gradient on the manifold is large and the probability of exciting modes perpendicular to $\mathcal{M}_{10}^{\mathrm{HH}}$ and $\mathcal{M}_{10}^{\mathrm{REP}}$ is not negligeable. It seems clear to us that more than 10 collective coordinates are needed to describe the system in these channels. On the other hand, it seems reasonable that if the system has low enough energy, it will all by itself steer clear of these regions and will 
not "feel" the existence of these higher energy states. It also seems physically sound that the system will spend most of its time near $\mathcal{M}_{10}^{\mathrm{MAC}}$. There the energy gradients are much smaller (there is about a $4 \%$ difference in energy between the torus state and a pair of infinitely separated hedgehogs). The manifold $\mathcal{M}_{10}^{\mathrm{MAC}}$ has been extensively studied by Leese et al [59] in connection with the study of the deuteron system.

The product ansatz in the most attractive channel possesses the following discrete symmetries at infinite separation (for two well separated Skyrmions on the $z$ axis, equidistant from the origin with one rotated relative to the other by $180^{\circ}$ about the $y$ axis):

$$
\begin{aligned}
& \vec{\pi}(-x, y, z)=\left(\begin{array}{ccc}
-1 & 0 & 0 \\
0 & 1 & 0 \\
0 & 0 & 1
\end{array}\right) \vec{\pi}(x, y, z) \\
& \vec{\pi}(x,-y, z)=\left(\begin{array}{ccc}
1 & 0 & 0 \\
0 & -1 & 0 \\
0 & 0 & 1
\end{array}\right) \vec{\pi}(x, y, z) \\
& \vec{\pi}(x, y,-z)=\left(\begin{array}{ccc}
-1 & 0 & 0 \\
0 & 1 & 0 \\
0 & 0 & 1
\end{array}\right) \vec{\pi}(x, y, z) .
\end{aligned}
$$

The last symmetry is actually valid for any separation while the first two are approximate and are exact only in the $R \rightarrow+\infty$ limit. These discrete symmetries have been shown to be exact for all configurations of $\mathcal{M}_{10}^{\mathrm{MAC}}$ including the torus, because they are conserved by the dynamics starting from $R=+\infty$ in the most attractive channel.

As the Skyrmions merge into the torus, the separation coordinate $R$ approaches a minimum value which depends on the definition for the separation chosen (which is always arbitrary to a certain point, since when the Skyrmions deform and come close together, the definition of separation becomes blurred). Another collective coordinate is effectively eliminated by the appearance of the axial symmetry of the torus 100:

$$
U_{\text {torus }}(\vec{x})=e^{\mathrm{i} \theta \tau^{3}} U_{\text {torus }}\left(D\left(e^{-\mathrm{i} \frac{\theta}{2} \tau^{3}}\right) \cdot \vec{x}\right) e^{-\mathrm{i} \theta \tau^{3}} .
$$

This axial symmetry is created by the dynamics of the system and generalises the discrete symmetries of equations (335), (336) and (337). This implies that the torus is described by only 8 collective coordinates which parametrize an 8-dimensional manifold named $\mathcal{M}_{8}$. Manton shows that one can describe the topology of $\mathcal{M}_{8}$ somewhat more precisely. Let us factor out global translations and global isorotations from $\mathcal{M}_{8}$, which are represented by a factor of $\mathbb{R}^{3} \times$ 
$S^{3}$. We are then left with 2 Euler angles, which parametrize a 2-sphere. The appearance of such an $S^{2}$ on a manifold is called a Bolt, in the theory of gravity [26, 31, 101]. A Bolt is a kind of "soft" singularity on a manifold. Atiyah and Manton further argue that because of the reflection symmetry of the torus relative to the plane perpendicular to the axis of the torus (the plane which "slices" the torus in two identical halves modulo a reflexion), the 2 -sphere is really an $\mathbb{R} P_{2}$ : a sphere with its antipodal points identified. This means that the two Euler angles over-define the orientation of the axis of the torus. This is topologically similar to the BPS case where a torus state also exists in the winding number (magnetic charge) 2 sector.

The dibaryon is at the center of the most exotic dynamics of the $B=2$ sector. Because of its hedgehog structure, see equation (304), it has the same 6 zero modes as the $B=1$ Skyrmion. Like all solutions of the theory, it also has positive modes corresponding to small oscillations about the critical point. However, it also possesses negative modes since it is a saddle point of the theory. It has long been suspected that the dibaryon is able to disintegrate into a pair of Skyrmions or into the torus itself; this was numerically checked only recently by Waindzoch and Wambach[91]. In their articles they use a discretized version of the dibaryon as the initial configuration and then perturbed it in order to study how the dibaryon is connected to the rest of the $B=2$ manifold. The negative modes of the dibaryon had already been investigated analytically by Bang and Wirzba 51 on a 3-sphere of radius $L$. The limit $L \rightarrow+\infty$ of their findings is in good agreement with those of the numerical simulations. They solve a Schrödinger-type equation for the perturbation of the dibaryon field in order to find the perturbations $\delta U$ which give the maximum negative energy gradients. This gave them three magnetic modes and three electric modes, whose names refer to the properties of transformation of the perturbation under rotations. We have already discussed these negative modes in section 3.2.3. The magnetic mode along the direction $z$ is parametrized as follows 91:

$$
\begin{aligned}
\delta U_{\mathrm{z}}^{\mathrm{M}}: \delta \pi^{0} & =0 \\
\delta \pi^{1} & =-g(r) \frac{y}{r} \\
\delta \pi^{2} & =g(r) \frac{x}{r} \\
\delta \pi^{3} & =0
\end{aligned}
$$

where $g(r)$ is a function computed numerically, the modes along the $x$ and $y$ axes being obtained by cyclic permutations. This mode possesses a simple interpretation when one recognizes that the dibaryon configuration is very close to a pair of Skyrmions in the product ansatz, placed one on top of the other. There are two ways to lower the energy of the resulting configuration: by translating or by rotating one Skyrmion relative to the other. The former represents the magnetic mode; there are three independent magnetic modes 
corresponding to the three orthogonal translation directions present in $\mathbb{R}^{3}$. The latter represents the electric mode which is parametrized by a somewhat more complicated form 91:

$$
\begin{aligned}
\delta U_{\mathrm{z}}^{\mathrm{E}}: \delta \pi^{0} & =-3 a(r) \frac{z}{r} \\
\delta \pi^{1} & =\left(c(r)-\frac{b(r)}{2}\right) \frac{z x}{r^{2}} \\
\delta \pi^{2} & =\left(c(r)-\frac{b(r)}{2}\right) \frac{z y}{r^{2}} \\
\delta \pi^{3} & =3\left(c(r)-\frac{b(r)}{2}\right) \frac{z^{2}}{r}+\frac{3}{2} b(r)
\end{aligned}
$$

for a perturbation along the $z$ axis (the profile functions $a(r), b(r)$ and $c(r)$ are obtained numerically). The other two can be obtained by cyclic permutations, $\delta U_{\mathrm{x}}^{\mathrm{E}}$ and $\delta U_{\mathrm{y}}^{\mathrm{E}}$ corresponding to rotations along the $x$ and $y$ axis respectively.

Ordinary time evolution of the dibaryon subject to these modes exhibits a fission process taking place. It can be numerically seen that for the perturbation $\delta U_{\mathrm{z}}^{\mathrm{M}}$, the dibaryon disintegrates in two Skyrmions moving along the $z$ axis. This negative mode is associated with a large energy gradient: the Skyrmions separate relatively quickly and oscillate strongly. The electric mode $\delta U^{\mathrm{E}}$ is slightly less energetic and the Skyrmions although moving away also along the $z$ axis, do so in a "twisting" fashion. Large oscillations are also present here. Waindzoch and Wambach also studied these same disintegrations using the gradient flow method. The oscillations, which are transverse to the low energy unstable manifold, are damped (see equations (261) and (262)) and it appears then that the magnetic mode is tangent to the path leading to the product ansatz in the $\mathrm{HH}$ channel, while the electric mode leads to the REP channel. This is still a delicate issue since the numerical computations are very hard to do and the precision required to confirm these results is quite high 91. Confirmation by analytic methods would be most welcome. Waindzoch and Wambach also show that the right superposition of perpendicular magnetic and electric perturbations can direct the system along the path leading straight to the toroidal configuration.

We are now ready to put the various parts together. Figure 28 shows schematically what the configuration space for the $B=2$ sector looks like. The 12-dimensional manifold $\mathcal{M}_{12}$ is the minimum required to describe the dynamics of a pair of Skyrmions or nucleons. As we saw earlier, for Skyrmions infinitely far from each other, these 12 collective coordinates can be identified as the individual positions and iso-orientations of the separate solitons. These states can be well represented by the product ansatz saddle point. The product ansatz comes in three different iso-orientations, if one chooses to freeze such 


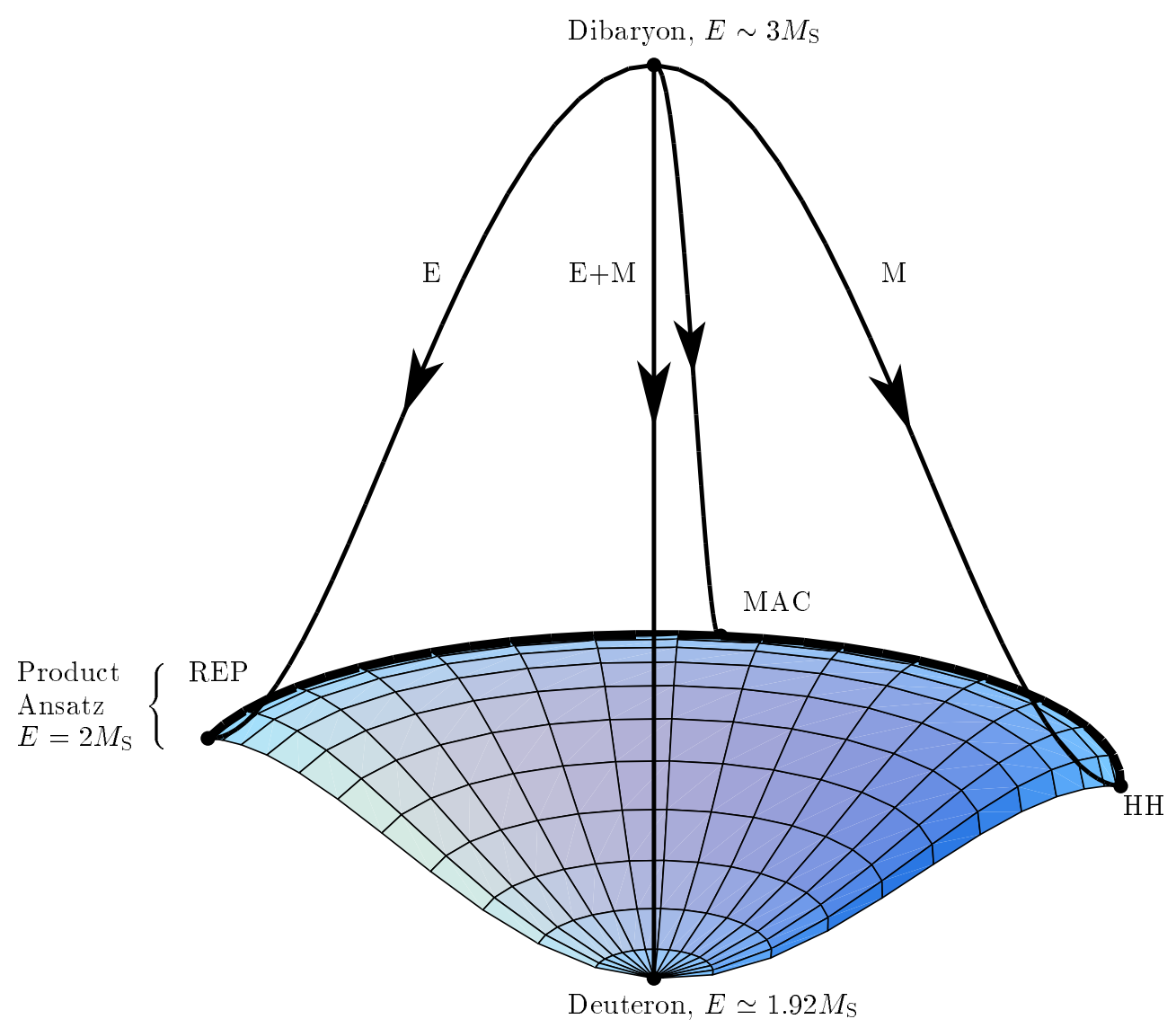

Fig. 28. Diagram of the $B=2$ manifold showing paths in configuration space connecting the dibaryon to the product ansatz in the HH and REP channels with paths tangent to the magnetic (M) and electric (E) modes respectively. The deuteron and the product ansatz MAC are connected to the dibaryon by paths comprising of an orthogonal superposition of $\mathrm{E}$ and $\mathrm{M}$ modes.

a degree of freedom: hedgehog-hedgehog channel, repulsive channel and the most attractive channel whose collective coordinates parametrize the manifolds $\mathcal{M}_{10}^{\mathrm{HH}}, \mathcal{M}_{10}^{\mathrm{REP}}$ and $\mathcal{M}_{10}^{\mathrm{MAC}}$ respectively. The product ansatz in the most attractive channel is linked to the toroidal configuration and its manifold $\mathcal{M}_{8}$ by gradient flow curves, while the $\mathrm{HH}$ and REP channels are connected to the dibaryon by paths whose directions near the dibaryon are tangent to its magnetic and electric modes respectively. The number of collective coordinates along those paths changes from 10 to 6 because of the appearance of the spherical symmetry of the dibaryon as well the freezing of the separation between the particles, removing 4 collective coordinates. The dibaryon is also directly connected to the torus as mentioned earlier by paths with initial directions spanned by the superposition of orthogonal magnetic and electric modes. Intermediate paths are generated by different combinations of these 6 modes. At the current rate of progress in this area, the relationship between 
the initial conditions and the final states attained should soon be clear. In the adiabatic approximation for Skyrmion dynamics, the paths can be followed in any direction (there is no pion wave emission here) but this is not physically plausible when energies differences of more than $1 \mathrm{GeV}$ are concerned.

\subsection{Low energy nucleon-nucleon scattering}

We now turn to the problem of nucleon-nucleon scattering as reported in Gisiger and Paranjape 39,40 following the methods proposed by Manton 37.

Scattering of Skyrmions at low energies should truncate to a dynamics taking place on an unstable manifold linking together all of the low energy critical points. We consider the scattering in a particularly simplifying approximation. One observes that as the Skyrmions are separated to infinity, their low-energy behaviour corresponds to two independent, free Skyrmions, each with 3 translational degrees of freedom and 3 rotational or isorotational degrees of freedom. The moduli space is simply two copies of $\mathbb{R}^{3} \times S^{3} / Z_{2}$ and the induced metric is also the natural metric on these manifolds. As the separation between the Skyrmions decreases, interactions develop between the Skyrmions, both in the potential and in the kinetic energy (,i.e., the metric). These interactions can be written in a systematic perturbative expansion in inverse powers of the separation. In what follows, we will only consider scattering at large impact parameter, for which the separation between the Skyrmions is always large. The perturbative expansion can therefore be truncated, and indeed our main approximation is to take only the leading term in this expansion. Surprisingly, this term comes from the expansion of the kinetic part of the Lagrangian and not the potential, and it has actually been overlooked by previous investigations. A secondary assumption that has been made and which we want to underline is that the pion mass is taken to be zero. This could perhaps explain why the interaction that we find has been missed: it comes from the two-pion exchange part of the interaction which is generally neglected when considering massive pions. We complete our analysis by using the Bohr-Sommerfeld quantization method and a modification of the method of the variation of constants to calculate the scattering trajectories of the nucleons.

\subsubsection{Lagrangian of the Skyrmion-Skyrmion system}

Written using the left-invariant one form already introduced previously (equation (14)), the Skyrme Lagrangian takes the form:

$$
\mathcal{L}_{s k}=\frac{f_{\pi}^{2}}{2} \mathcal{R}(U)_{\mu} \cdot \mathcal{R}(U)^{\mu}-\frac{1}{4 e^{2}}\left[\mathcal{R}(U)_{\mu} \cdot \mathcal{R}(U)^{\mu} \mathcal{R}(U)_{\nu} \cdot \mathcal{R}(U)^{\nu}-\right.
$$




$$
\left.\mathcal{R}(U)_{\mu} \cdot \mathcal{R}(U)^{\nu} \mathcal{R}(U)_{\nu} \cdot \mathcal{R}(U)^{\mu}\right]
$$

which, as we saw earlier, separates into a kinetic and a potential part:

$$
\begin{aligned}
& \mathcal{T}=\frac{f_{\pi}^{2}}{2} \mathcal{R}_{0} \cdot \mathcal{R}_{0}-\frac{1}{2 e^{2}}\left[\mathcal{R}_{0} \cdot \mathcal{R}_{\mathrm{i}} \mathcal{R}_{0} \cdot \mathcal{R}_{\mathrm{i}}-\mathcal{R}_{0} \cdot \mathcal{R}_{0} \mathcal{R}_{\mathrm{i}} \cdot \mathcal{R}_{\mathrm{i}}\right] \\
& \mathcal{V}=-\frac{f_{\pi}^{2}}{2} \mathcal{R}_{\mathrm{i}} \cdot \mathcal{R}_{\mathrm{i}}-\frac{1}{4 e^{2}}\left[\mathcal{R}_{\mathrm{i}} \cdot \mathcal{R}_{\mathrm{i}} \mathcal{R}_{\mathrm{j}} \cdot \mathcal{R}_{\mathrm{j}}-\mathcal{R}_{\mathrm{i}} \cdot \mathcal{R}_{\mathrm{j}} \mathcal{R}_{\mathrm{j}} \cdot \mathcal{R}_{\mathrm{i}}\right] .
\end{aligned}
$$

The potential part has been extensively studied in the literature. It produces the following leading order term in the separation $d$ between the Skyrmions [1,9, 35]:

$$
V=2 M+4 \pi f_{\pi}^{2} \kappa^{2} \frac{(1-\cos \theta)\left(3(\hat{n} \cdot \hat{d})^{2}-1\right)}{d^{3}}
$$

where $\hat{d}=\vec{d} / d$. We are interested by the kinetic part of the Lagrangian which is less understood.

As noted earlier, to accurately describe the motion of the pair of Skyrmions we need the metric on the unstable manifold of the baryon number 2 sector, as well as the potential defined on it. Since both are not well known one must resort to approximating the pair of Skyrmions by a parametrization or ansatz. We chose the simplest parametrization for the system, the product ansatz, which maintains both solitons rigidly in the Skyrmion configuration at all time. Of course, since the particles deform when they come close to each other, our parametrization is only valid when the Skyrmions are far from each other. This is compatible with the low energy assumption we made and which is necessary to ensure that the degrees of freedom of the system can indeed be truncated to a finite (small) number. Then one takes

$$
U(\vec{x})=U_{1}^{\dagger} U_{2}=A^{\dagger} U\left(\vec{x}-\vec{R}_{1}\right) A B^{\dagger} U\left(\vec{x}-\vec{R}_{2}\right) B
$$

$A$ and $B$ are time dependent $\mathrm{SU}(2)$ matrices representing the orientation of the Skyrmions and $\vec{R}_{1}$ and $\vec{R}_{2}$ are their respective positions. A symmetrized product ansatz is possible, which respects the symmetry under exchange of the two particles [96], however such an elaboration does not affect the first order term in the mutual interaction.

Replacing $U$ by $U_{1}^{\dagger} U_{2}$ in the kinetic part of the Skyrme Lagrangian (18) and working out the computations using the identity

$$
\mathcal{R}_{\mu}^{\mathrm{a}}\left(U_{1} U_{2}\right)=\mathcal{R}_{\mu}^{\mathrm{a}}\left(U_{1}\right)+D_{\mathrm{ab}}\left(U_{1}\right) \mathcal{R}_{\mu}^{\mathrm{b}}\left(U_{2}\right)
$$


where $D_{\mathrm{ab}}(U)$ is $\frac{1}{2} \operatorname{tr}\left[\tau^{\mathrm{a}} U \tau^{\mathrm{b}} U^{\dagger}\right]$, allows us to isolate the interaction term $\mathcal{T}_{\text {int }}$ from contributions describing free individual particles. The kinetic part of the Lagrangian then writes

$$
\mathcal{T}=\mathcal{T}_{1}+\mathcal{T}_{2}+\mathcal{T}_{\text {int }}
$$

where

$$
\begin{aligned}
& \mathcal{T}_{\text {int }}=f_{\pi}^{2} \mathcal{R}_{0}^{1} \cdot D \cdot \mathcal{R}_{0}^{2} \\
& +\frac{1}{2 e^{2}}\left[\left(\mathcal{R}_{0}^{1} \cdot \mathcal{R}_{0}^{1} \mathcal{R}_{\mathrm{i}}^{2} \cdot \mathcal{R}_{\mathrm{i}}^{2}+1 \leftrightarrow 2\right)+2\left(\mathcal{R}_{0}^{1} \cdot \mathcal{R}_{0}^{1}+1 \leftrightarrow 2\right) \mathcal{R}_{\mathrm{i}}^{1} \cdot D \cdot \mathcal{R}_{\mathrm{i}}^{2}\right. \\
& +2\left(\mathcal{R}_{\mathrm{i}}^{1} \cdot \mathcal{R}_{\mathrm{i}}^{2}+1 \leftrightarrow 2\right) \mathcal{R}_{0}^{1} \cdot D \cdot \mathcal{R}_{0}^{2}+4 \mathcal{R}_{0}^{1} \cdot D \cdot \mathcal{R}_{0}^{2} \mathcal{R}_{\mathrm{i}}^{1} \cdot D \cdot \mathcal{R}_{\mathrm{i}}^{2} \\
& -\left(\mathcal{R}_{0}^{1} \cdot D \cdot \mathcal{R}_{\mathrm{i}}^{2}\right)^{2}-\left(\mathcal{R}_{\mathrm{i}}^{1} \cdot D \cdot \mathcal{R}_{0}^{2}\right)^{2}-2 \mathcal{R}_{0}^{1} \cdot \mathcal{R}_{\mathrm{i}}^{1} \mathcal{R}_{0}^{2} \cdot \mathcal{R}_{\mathrm{i}}^{2} \\
& -2 \mathcal{R}_{0}^{1} \cdot D \cdot \mathcal{R}_{\mathrm{i}}^{2} \mathcal{R}_{\mathrm{i}}^{1} \cdot D \cdot \mathcal{R}_{0}^{2} \\
& \left.-2\left(\mathcal{R}_{0}^{1} \cdot \mathcal{R}_{i}^{1}+1 \leftrightarrow 2\right)\left(\mathcal{R}_{0}^{1} \cdot D \cdot \mathcal{R}_{\mathrm{i}}^{2}+\mathcal{R}_{\mathrm{i}}^{1} \cdot D \cdot \mathcal{R}_{0}^{2}\right)\right]
\end{aligned}
$$

where the dot implies contraction over isospin, $\mathcal{R}_{\mu}^{1} \equiv \mathcal{R}_{\mu}^{\mathrm{a}}\left(U_{1}\right), \mathcal{R}_{\mu}^{2} \equiv \mathcal{R}_{\mu}^{\mathrm{a}}\left(U_{2}\right)$ and $D \equiv D_{\mathrm{ab}}\left(U_{1}\right)$ and $\mathcal{T}_{1}$ and $\mathcal{T}_{2}$ are similar to (18) for the fields $U_{1}$ and $U_{2}$ respectively.

To obtain the full Lagrangian of the system we need to integrate the Lagrangian density over all space:

$$
L_{\text {int }}=\int \mathrm{d}^{3} \vec{x} \mathcal{L}_{\text {int }}
$$

Since the Skyrmion profile function $f(r)$ is only known numerically, even though it falls to zero like $1 / r^{2}$ for large $r$, it is not possible to compute the integral analytically. An expansion in inverse powers of the separation $d=\left\|\vec{R}_{1}-\vec{R}_{2}\right\|$ between the Skyrmions is then used ( $d$ was assumed to be large from the start in order for the product ansatz to be accurate).

To do this we first write $U_{1}$ and $U_{2}$ as functions of the matrices $A$ and $B$ and the position vectors $\vec{R}_{1}$ and $\vec{R}_{2}$ using (351) and the following identity:

$$
\begin{aligned}
\mathcal{R}_{0}^{\mathrm{a}}\left(U_{1}\right)=\left(\delta^{\mathrm{ab}}-D_{\mathrm{ab}}\left(A U\left(\vec{x}-\vec{R}_{1}\right) A^{\dagger}\right)\right) \mathcal{R}_{0}^{\mathrm{a}}(A)- \\
D_{\mathrm{ab}}(A) \dot{R_{1}^{\mathrm{i}}} \mathcal{R}_{\mathrm{i}}^{\mathrm{b}}\left(U\left(\vec{x}-\vec{R}_{1}\right)\right)
\end{aligned}
$$

and correspondingly for $U_{2}, B$ and $\vec{R}_{2}$. We are interested in the leading contributions in inverse powers of $d$ of these tensors. Since the Skyrmion profile 
function $f(r)$ behaves like $\kappa / r^{2}$ at large separation, we get after a short calculation for large values of $\left|\vec{x}-\vec{R}_{1}\right|$ :

$$
\begin{aligned}
\mathcal{D}_{\mathrm{ab}}\left(U_{1}\right) & \equiv \delta^{\mathrm{ab}}-D_{\mathrm{ab}}\left(A U\left(\vec{x}-\vec{R}_{1}\right) A^{\dagger}\right) \\
& =-2 \kappa \frac{\epsilon^{\mathrm{abc}} D_{\mathrm{cd}}(A) \hat{r}_{1}^{\mathrm{d}}}{\left|\vec{x}-\vec{R}_{1}\right|^{2}}+O\left(\frac{1}{\left|\vec{x}-\vec{R}_{1}\right|^{3}}\right)
\end{aligned}
$$

and

$$
\mathcal{R}_{\mathrm{i}}^{\mathrm{a}}(A)=\frac{\kappa}{\left|\vec{x}-\vec{R}_{1}\right|^{3}}\left(\delta^{\mathrm{ia}}-3 \hat{r}_{1}^{\mathrm{i}} \hat{r}_{1}^{\mathrm{a}}\right)+O\left(\frac{1}{\left|\vec{x}-\vec{R}_{1}\right|^{4}}\right)
$$

where $\hat{r}_{1}=\left(\vec{x}-\vec{R}_{1}\right) /\left|\vec{x}-\vec{R}_{1}\right|$, and correspondingly for $U_{2}, B$ and $\vec{R}_{2}$. To go further we will use a standard method for this type of calculation (for a very complete description of this method, its subtleties and its application to the computation of the Skyrmion-Skyrmion potential in the product ansatz see [35).

In this method, we divide space in three regions I, II and C. I and II are regions of space close to the Skyrmions 1 and 2 respectively (see figure 29). We define the notion of closeness by stating that these are regions where the profile function $f(r)$ is not well approximated by its asymptotic expression. For simplicity, and taking advantage of the spherical symmetry of the Skyrmion, we will take those regions to be spheres of some chosen radius $R$. Region $\mathrm{C}$ is the complementary region where both Skyrmion profiles fall off like $1 /\left(\vec{R}_{\mathrm{i}}-\vec{r}\right)^{2}$ $(i=1,2)$. We then evaluate the integral over each region separately using the fact that in regions I and II, one can use the "exact" expression for the Skyrmion profile, while in region $\mathrm{C}$ both Skyrmion profiles behave according to their asymptotic forms.

Let us start with the contributions from the non-linear $\sigma$ model, i.e. the term quadratic in time derivatives. By using (17) and (356) we easily find that:

$$
\begin{aligned}
\mathcal{R}_{\mathrm{a}}^{0}\left(U_{1}\right) D_{\mathrm{ab}}\left(U_{1}\right) \mathcal{R}_{\mathrm{c}}^{0}\left(U_{2}\right)= \\
\quad \mathcal{R}_{0}^{\mathrm{c}}(A) \mathcal{D}_{\mathrm{ca}}\left(U_{1}^{\dagger}\right) D_{\mathrm{ab}}\left(U_{1}\right) \mathcal{D}_{\mathrm{bd}}\left(U_{2}\right) \mathcal{R}_{0}^{\mathrm{d}}(B) \\
-\mathcal{R}_{0}^{\mathrm{c}}(A) \mathcal{D}_{\mathrm{ca}}\left(U_{1}^{\dagger}\right) D_{\mathrm{ab}}\left(U_{1}\right) D_{\mathrm{bd}}(B) \dot{R}_{2}^{\mathrm{j}} \mathcal{R}_{\mathrm{j}}^{\mathrm{d}}\left(U\left(\vec{x}-\vec{R}_{2}\right)\right) \\
-\mathcal{R}_{\mathrm{i}}^{\mathrm{c}}\left(U\left(\vec{x}-\vec{R}_{1}\right)\right) \dot{R}_{1}^{\mathrm{i}} D_{\mathrm{ca}}\left(A^{\dagger}\right) D_{\mathrm{ab}}\left(U_{1}\right) \mathcal{D}_{\mathrm{bd}}\left(U_{2}\right) \mathcal{R}_{0}^{\mathrm{d}}(B) \\
+\mathcal{R}_{\mathrm{i}}^{\mathrm{c}}\left(U\left(\vec{x}-\vec{R}_{1}\right)\right) \dot{R}_{1}^{\mathrm{i}} D_{\mathrm{ca}}\left(A^{\dagger}\right) D_{\mathrm{ab}}\left(U_{1}\right) D_{\mathrm{bd}}(B) \dot{R}_{2}^{\mathrm{j}} \mathcal{R}_{\mathrm{j}}^{\mathrm{d}}\left(U\left(\vec{x}-\vec{R}_{2}\right)\right)
\end{aligned}
$$

When integrating this expression over region I we can use the asymptotic expansion for the tensors of Skyrmion 2, giving 


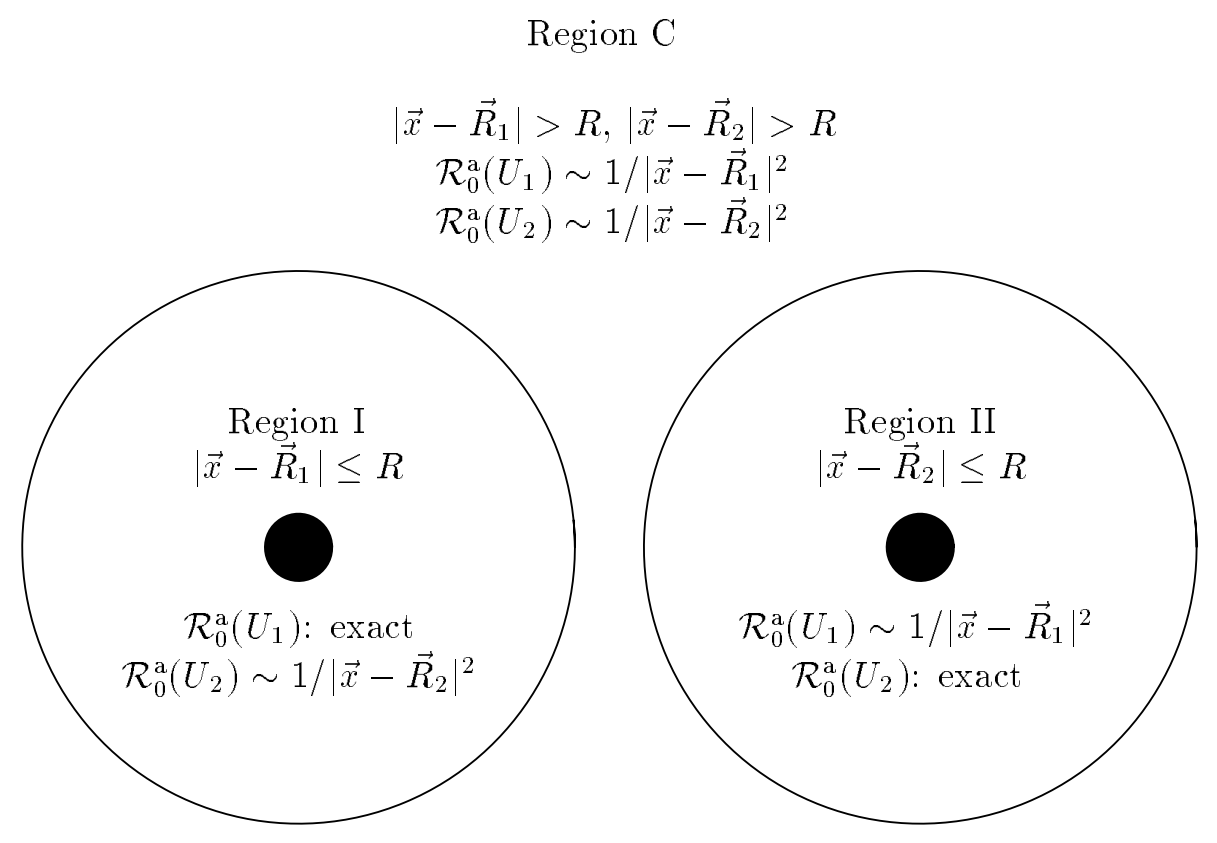

Fig. 29. Diagram of the three regions I, II and C used to divide space in order to compute the Lagrangian of a pair of Skyrmions as an expansion in inverse powers of the separation between the particles

$$
\begin{aligned}
& \int_{\mathrm{I}} \mathrm{d}^{3} \vec{x} \mathcal{R}_{\mathrm{a}}^{0}\left(U_{1}\right) D_{\mathrm{ab}}\left(U_{1}\right) \mathcal{R}_{\mathrm{b}}^{0}\left(U_{2}\right)= \\
& -2 \kappa \int_{\left|\vec{x}-\vec{R}_{1}\right| \leq R} \mathrm{~d}^{3} \vec{x} \frac{\mathcal{R}_{0}^{\mathrm{a}}(A) \mathcal{D}_{\mathrm{ab}}\left(U_{1}^{\dagger}\right) D_{\mathrm{bc}}\left(U_{1}\right) \epsilon^{\mathrm{cde}} D_{\mathrm{ef}}(B) \hat{r}_{2}^{\mathrm{f}} \mathcal{R}_{0}^{\mathrm{d}}(B)}{\left|\vec{x}-\vec{R}_{2}\right|^{2}}+ \\
& +2 \kappa \int_{\left|\vec{x}-\vec{R}_{1}\right| \leq R} \mathrm{~d}^{3} \vec{x} \frac{\mathcal{R}_{\mathrm{i}}^{\mathrm{a}}\left(U\left(\vec{x}-\vec{R}_{1}\right)\right) \dot{R}_{1}^{\mathrm{i}} D_{\mathrm{ab}}\left(A^{\dagger}\right) D_{\mathrm{bc}}\left(U_{1}\right) \epsilon^{\mathrm{cde}} D_{\mathrm{ef}}(B) \hat{r}_{2}^{\mathrm{f}} \mathcal{R}_{0}^{\mathrm{d}}(B)}{\left|\vec{x}-\vec{R}_{2}\right|^{2}} \\
& \quad+O\left(\frac{1}{\left|\vec{x}-\vec{R}_{2}\right|^{3}}\right) .
\end{aligned}
$$

By translating the integration variable by $\vec{R}_{1}$, and defining a new integration variable $\vec{y}=\vec{x}-\vec{R}_{1}$, we easily see that this integral contributes at most terms of order $1 / d^{2}$. Integration over region II yields the same result by symmetry. Integrating over the complementary region $\mathrm{C}$ is different since tensors from both Skyrmions take their asymptotic form and we find to leading order:

$$
\int_{\mathrm{C}} \mathrm{d}^{3} \vec{x} \mathcal{R}_{\mathrm{a}}^{0}\left(U_{1}\right) D_{\mathrm{ab}}\left(U_{1}\right) \mathcal{R}_{\mathrm{b}}^{0}\left(U_{2}\right)=
$$




$$
\begin{aligned}
& 4 \kappa^{2} \int_{\left|\vec{x}-\vec{R}_{1}\right| \geq R,\left|\vec{x}-\vec{R}_{2}\right| \geq R} \mathrm{~d}^{3} \vec{x} \frac{\epsilon^{\mathrm{aeb}} D_{\mathrm{ef}}(A) \hat{r}_{1}^{\mathrm{f}} \mathcal{R}_{0}^{\mathrm{b}}(A) \epsilon^{\mathrm{adg}} \mathcal{R}_{0}^{\mathrm{d}}(B) D_{\mathrm{gh}}(B) \hat{r}_{2}^{\mathrm{h}}}{\left|\vec{x}-\vec{R}_{1}\right|^{2}\left|\vec{x}-\vec{R}_{2}\right|^{2}}+\ldots \\
& =4 \kappa^{2} \int_{\mathrm{I}+\mathrm{II}+\mathrm{C}} \mathrm{d}^{3} \vec{y} \frac{\mathcal{R}_{0}^{\mathrm{a}}(A) \mathcal{R}_{0}^{\mathrm{b}}(B)\left[D_{\mathrm{ac}}(B) \hat{y}^{\mathrm{c}} D_{\mathrm{bd}}(A)(\vec{y}-\vec{d})^{\mathrm{d}}-(\vec{y}-\vec{d})^{\mathrm{c}} D_{\mathrm{cd}}\left(A^{\dagger} B\right) \hat{y}^{\mathrm{d}} \delta^{\mathrm{ab}}\right]}{y^{2}|\vec{y}-\vec{d}|^{2}} \\
& +\ldots \\
& =\frac{4 \kappa^{2}}{d} \int \mathrm{d}^{3} \vec{z} \frac{\mathcal{R}_{0}^{\mathrm{a}}(A) \mathcal{R}_{0}^{\mathrm{b}}(B)\left[D_{\mathrm{ac}}(B) \hat{z}^{\mathrm{c}} D_{\mathrm{bd}}(A)(\vec{z}-\hat{d})^{\mathrm{d}}-(\overrightarrow{\vec{z}-\hat{d})})^{\mathrm{c}} D \mathrm{~cd}\left(A^{\dagger} B\right) \hat{z}^{\mathrm{d}} \delta^{\mathrm{ab}}\right]}{z^{2}|\vec{z}-\hat{d}|^{2}} \\
& +O\left(\frac{1}{d^{2}}\right)
\end{aligned}
$$

where $\vec{y}=\vec{x}-\vec{R}_{2}$ and $\vec{z}=\vec{y} / d$. To obtain (363), we used the fact that expanding the integration bounds from $\mathrm{C}$ to the whole space only adds higher order contributions to the integral, but does not alter the value of the leading order terms (see 35 for details). The last line (364) is obtained by shifting the integration variable by $R_{1}$ and absorbing 3 factors of $d$ in the measure. This is possible since we are integrating over all space.

The fact that the main contribution comes from the faraway region might at first sight seem a bit strange, but it should be kept in mind that this same region makes a contribution in the analogous computation of the potential (350), which is as important as the contribution from the regions close to the Skyrmions (see [35] for more details on that point).

Similar computations are feasible for the Skyrme term, but because of its structure (quartic in derivatives), it only contributes at higher order. The leading contribution from the kinetic part of the Lagrangian is then of order $1 / d$ while the potential part is of order $1 / d^{3}$ rendering the latter, in principle, negligible compared to the contribution from the kinetic energy. Finally we obtain the following expansion of the Lagrangian for a pair of Skyrmions far from each other in the product ansatz, using the expression (21) for single Skyrmion Lagrangians:

$$
\begin{array}{rl}
L=-2 & M+\frac{1}{4} M \dot{\vec{d}}^{2}+2 \Lambda\left(\mathcal{R}^{\mathrm{a}}(A) \mathcal{R}^{\mathrm{a}}(A)+\mathcal{R}^{\mathrm{a}}(B) \mathcal{R}^{\mathrm{a}}(B)\right) \\
+ & \frac{\Delta}{d} \epsilon^{\mathrm{iac}} \epsilon^{\mathrm{jbd}} \mathcal{L}^{\mathrm{a}}(A) \mathcal{L}^{\mathrm{d}}(B)\left(\delta^{\mathrm{ij}}-\hat{d}^{\mathrm{i}} \hat{d}^{\mathrm{j}}\right) D_{\mathrm{ab}}\left(A^{\dagger} B\right)+O\left(1 / d^{2}\right)
\end{array}
$$

where $\hat{d}=\vec{d} / d, \Delta=2 \pi \kappa^{2} f_{\pi}^{2} \cdot \mathcal{L}^{\mathrm{a}}(A) \equiv \mathcal{L}_{0}^{\mathrm{a}}(A), \mathcal{R}^{\mathrm{a}}(A) \equiv \mathcal{R}_{0}^{\mathrm{a}}(A)$ and we have used the relation $\mathcal{L}^{\mathrm{a}}(A)=D_{\mathrm{ab}}(A) \mathcal{R}^{\mathrm{b}}(A)$.

By keeping only the leading order terms in $1 / d$, we neglect any contribution from the potential part of the original Lagrangian, and the motion is then 
completely specified by the geodesics of the metric induced by the kinetic term onto the unstable manifold of the baryon number two sector, as specified in subsection 4.3 .

The term in $1 / d$ in (365), though absent from the literature of the 80's, was also independently obtained by Schroers 41. He found a leading contribution which behaves as $1 / d$ and even calculated sub-leading spin-orbit coupling terms. The only other comparable calculation to our knowledge has been done by Walhout and Wambach [20] for the case of massive pions. The limit as $m_{\pi} \rightarrow 0$ of their expression, however, does not leave a term which behaves as $1 / d$ and hence does not reproduce our result. We believe that this contribution should come also from their evaluation of the integral giving the induced kinetic energy in the faraway region (region $\mathrm{C}$ ) and then recovering our result as $m_{\pi} \rightarrow 0$. Such a contribution would also be proportional to $\left(e^{-m_{\pi} d}\right)^{2}$, what they call "two pion exchange". We also add that this $1 / d$ term is of leading order in an expansion in inverse separation with respect to a scale determined by $f_{\pi}$ and $e$ which has nothing to do with the length scale set by the pion mass.

The Skyrmion-Skyrmion Lagrangian we obtained describes dynamics in a 12dimensional moduli space via equations of motion which are highly non-linear and quite complex. The Lagrangian possesses several symmetries, and associated conserved quantities such as total isospin which is connected to invariance under left isorotation

$$
A \rightarrow C A \quad \text { and } \quad B \rightarrow C B
$$

and the total angular momentum related to invariance under the following operation:

$$
A \rightarrow A C \quad \text { and } \quad B \rightarrow B C \quad \text { and } \quad d^{\mathrm{a}} \rightarrow D_{\mathrm{ab}}\left(C^{\dagger}\right) d^{\mathrm{b}}
$$

(where $C$ is a constant $S U(2)$ matrix). However they are not much help in simplifying the equations of motion or even solving them. In order to go further, and to keep numerical analysis to a minimum, we use the perturbation method of Lagrange 90 familiar in celestial mechanics to compute approximations to the equations of motion. We will describe how this perturbative scheme works.

We are dealing here with a system described by a Lagrangian of purely kinetic nature which we will note:

$$
\begin{aligned}
L & =T_{1}+T_{2}+T_{\mathrm{I}} \\
& \equiv T_{0}+T_{\mathrm{I}}
\end{aligned}
$$


where $T_{0}=T_{1}+T_{2}$ is the free Lagrangian, $T_{\mathrm{I}}$ is the interaction part and $T_{\mathrm{I}} \ll T_{0}$ because of some small factor in $T_{\mathrm{I}}\left(1 / d\right.$ in this case). Let $q_{\mathrm{i}}^{0}$ denote the free coordinates of the system, which we take to completely describe its state. In our case $q_{\mathrm{i}}^{0}$ will represent $d^{\mathrm{i}}, A$ or $B$. The free canonically conjugate variables are defined as

$$
p_{\mathrm{i}}^{0}=\frac{\partial}{\partial \dot{q}_{\mathrm{i}}^{0}} T_{0}\left(q_{\mathrm{i}}^{0}, \dot{q}_{\mathrm{i}}^{0}\right)
$$

Here $\dot{q}_{\mathrm{i}}^{0}$ are taken to be $\dot{d}^{\mathrm{i}}, \mathcal{R}^{\mathrm{i}}(A), \mathcal{R}^{\mathrm{i}}(B), \mathcal{L}^{\mathrm{i}}(A)$ and $\mathcal{L}^{\mathrm{i}}(B)$. In the free system, which is described by $T_{0}$, these quantities are conserved. Indeed, without the interaction term, the Lagrangian describes a pair of free, spherically symmetrical tops moving and spinning at constant velocity.

Adding $T_{\mathrm{I}}$ to $T_{0}$ complicates things and removes those conservation laws. Even though the system is still described by the same coordinates $q_{\mathrm{i}}=q_{\mathrm{i}}^{0}$, the canonically conjugate variables are changed; we find

$$
\begin{aligned}
p_{\mathrm{i}} & =\frac{\partial}{\partial \dot{q}_{\mathrm{i}}}\left(T_{0}\left(q_{\mathrm{i}}, \dot{q}_{\mathrm{i}}\right)+T_{\mathrm{I}}\left(q_{\mathrm{i}}, \dot{q}_{\mathrm{i}}\right)\right) \\
& =p_{\mathrm{i}}\left(q_{\mathrm{j}}^{0}, p_{\mathrm{j}}^{0}\right)=p_{\mathrm{i}}^{0}+\Delta p_{\mathrm{i}}
\end{aligned}
$$

where $\Delta p_{\text {i }}$ has a well defined expansion in $1 / d$. The aim of the method is to find an accurate expansion in $1 / d$ of the equations of motion of the system, enabling one to only keep the dominant terms. This is done using Poisson brackets. Let us denote by $C^{\mathrm{k}}\left(q^{0}, p^{0}\right)$ quantitites which are conserved in the free system. In our case, they will be $\dot{d}_{\mathrm{i}}, \mathcal{R}^{\mathrm{i}}(A), \mathcal{R}^{\mathrm{i}}(B), \mathcal{L}^{\mathrm{i}}(A)$ and $\mathcal{L}^{\mathrm{i}}(B)$ but they could also represent more complicated functions of these quantities. $C^{\mathrm{k}}(q, p)$ will no longer necessarily be conserved and they can be separated in a free and an interacting part as follows:

$$
\begin{aligned}
C^{\mathrm{k}}\left(q_{\mathrm{i}}, p_{\mathrm{i}}\right) & =C^{\mathrm{k}}\left(q_{\mathrm{i}}, p_{\mathrm{i}}^{0}+\Delta p_{\mathrm{i}}\right) \\
& \simeq C^{\mathrm{k}}\left(q_{\mathrm{i}}^{0}, p_{\mathrm{i}}^{0}\right)+\frac{\partial}{\partial p_{\mathrm{i}}^{0}} C^{\mathrm{k}}\left(q_{\mathrm{i}}^{0}, p_{\mathrm{i}}^{0}\right) \Delta p_{\mathrm{i}} .
\end{aligned}
$$

The interacting part can be written in a $1 / d$ expansion. The time derivative of $C^{\mathrm{k}}$ is given by the Poisson bracket of $C^{\mathrm{k}}$ with the Hamiltonian of the system:

$$
\frac{\mathrm{d}}{\mathrm{dt}} C^{\mathrm{k}}=\left\{C^{\mathrm{k}}, H\right\}
$$

where $H=H_{0}+H_{\mathrm{I}}=\sum_{\mathrm{i}} q_{\mathrm{i}} p_{\mathrm{i}}-\left(T_{0}+T_{\mathrm{I}}\right)$. $H_{\mathrm{I}}$ being suppressed by a factor of $1 / d$ relative to $H_{0}$, writing $H$ as $H_{0}+H_{\mathrm{I}}$ also provides an expansion in 
this small parameter. Finally the Poisson bracket itself can be written as an expansion in $1 / d$. Indeed, since:

$$
\frac{\partial}{\partial q_{i}}=\frac{\partial}{\partial q_{i}^{0}} \quad \text { and } \quad \frac{\partial}{\partial p_{i}}=\sum_{j}\left(\frac{\partial p_{j}^{0}}{\partial p_{i}}\right) \frac{\partial}{\partial p_{j}^{0}}=\frac{\partial}{\partial p_{i}^{0}}-\sum_{j}\left(\frac{\partial \Delta p_{j}}{\partial p_{i}}\right) \frac{\partial}{\partial p_{j}^{0}}
$$

then

$$
\begin{aligned}
& \{A(q, p), B(q, p)\}=\sum_{\mathrm{i}} \frac{\partial A(q, p)}{\partial q_{\mathrm{i}}} \frac{\partial B(q, p)}{\partial p_{\mathrm{i}}}-\frac{\partial A(q, p)}{\partial p_{\mathrm{i}}} \frac{\partial B(q, p)}{\partial q_{\mathrm{i}}} \\
& =\left.\sum_{\mathrm{i}}\left[\frac{\partial A}{\partial q_{\mathrm{i}}^{0}} \frac{\partial B}{\partial p_{\mathrm{i}}^{0}}-\frac{\partial A}{\partial p_{\mathrm{i}}^{0}} \frac{\partial B}{\partial q_{\mathrm{i}}^{0}}-\sum_{\mathrm{j}}\left(\frac{\partial A}{\partial q_{\mathrm{i}}^{0}} \frac{\partial B}{\partial p_{\mathrm{i}}^{0}}-\frac{\partial A}{\partial p_{\mathrm{i}}^{0}} \frac{\partial B}{\partial q_{\mathrm{i}}^{0}}\right)\left(\frac{\partial \Delta p_{\mathrm{j}}}{\partial p_{\mathrm{i}}}\right)\right]\right|_{\left(q^{0}, p^{0}+\Delta p\right)} \\
& =\{A, B\}_{0}-\sum_{\mathrm{i}, \mathrm{j}}\left(\frac{\partial A}{\partial q_{\mathrm{i}}^{0}} \frac{\partial B}{\partial p_{\mathrm{j}}^{0}}-\frac{\partial A}{\partial p_{\mathrm{i}}^{0}} \frac{\partial B}{\partial q_{\mathrm{i}}^{0}}\right)\left(\frac{\partial \Delta p_{\mathrm{j}}}{\partial p_{\mathrm{i}}}\right) \\
& \equiv\{A, B\}_{0}+\{A, B\}_{\mathrm{I}}
\end{aligned}
$$

Replacing these expressions in (372), we find the following expansion in $1 / d$ of the time derivative of $C^{\mathrm{k}}$ :

$$
\begin{aligned}
\frac{\mathrm{d}}{\mathrm{dt}} C^{\mathrm{k}}= & \left\{C^{k}\left(q^{0}, p^{0}\right), H_{0}\left(q^{0}, p^{0}\right)\right\}_{\mathrm{I}}+\left\{C^{\mathrm{k}}\left(q^{0}, p^{0}\right), H_{\mathrm{I}}\left(q^{0}, p^{0}\right)\right\}_{0}+ \\
& \left\{\frac{\partial}{\partial p_{\mathrm{i}}^{0}} C^{\mathrm{k}}\left(q^{0}, p^{0}\right) \Delta p_{\mathrm{i}}, H_{0}\left(q^{0}, p^{0}\right)\right\}_{0}+ \\
& \left\{C^{\mathrm{k}}\left(q^{0}, p^{0}\right), \frac{\partial}{\partial p_{\mathrm{i}}^{0}} H_{0}\left(q^{0}, p^{0}\right) \Delta p_{\mathrm{i}}\right\}_{0}+\cdots
\end{aligned}
$$

since $\left\{C^{\mathrm{k}}, H_{\mathrm{I}}\right\}_{\mathrm{I}}$ is automatically of higher order in $1 / d$ and $\left\{C^{\mathrm{k}}, H_{0}\right\}_{0}=0$ exactly. The only asumption made here is that $d$ is large.

For the case $C^{\mathrm{k}}=\dot{d}^{\mathrm{k}}$, since $H_{\mathrm{I}}$ does not depend on $\dot{d}^{\mathrm{k}}$, the last three terms in (375) vanish, thus

$$
\begin{aligned}
& \frac{\mathrm{d}}{\mathrm{dt}} \dot{d}^{\mathrm{k}}=\left\{\dot{d}^{\mathrm{k}}, H_{\mathrm{I}}\right\}_{0} \\
&=-\frac{2 \Delta}{M d^{2}}\left[\delta^{\mathrm{ij}} \hat{d}^{\mathrm{k}}+\delta^{\mathrm{jk}} \hat{d}^{\mathrm{i}}+\delta^{\mathrm{ik}} \hat{d}^{\mathrm{j}}-3 \hat{d}^{\mathrm{i}} \hat{d}^{\mathrm{j}} \hat{d}^{\mathrm{k}}\right] \times \\
& \epsilon^{\mathrm{iac}} \epsilon^{\mathrm{jbd}} \mathcal{L}^{\mathrm{c}}(A) \mathcal{L}^{\mathrm{d}}(B) D_{\mathrm{ab}}\left(A^{\dagger} B\right)
\end{aligned}
$$

using the free Poisson brackets of the free system:

$$
\left\{d^{\mathrm{i}}, \Pi^{\mathrm{j}}\right\}=\delta^{\mathrm{ij}}
$$




$$
\begin{aligned}
\left\{\mathcal{R}^{\mathrm{a}}(A), \mathcal{R}^{\mathrm{b}}(A)\right\} & =-\frac{1}{2 \Lambda} \epsilon^{\mathrm{abc}} \mathcal{R}^{\mathrm{c}}(A) \\
\left\{\mathcal{L}^{\mathrm{a}}(A), \mathcal{L}^{\mathrm{b}}(A)\right\} & =\frac{1}{2 \Lambda} \epsilon^{\mathrm{abc}} \mathcal{L}^{\mathrm{c}}(A) \\
\left\{\mathcal{R}^{\mathrm{a}}(A), \mathcal{L}^{\mathrm{b}}(A)\right\} & =0 \\
\left\{\mathcal{R}^{\mathrm{a}}(A), D_{\mathrm{bc}}(A)\right\} & =-\frac{1}{2 \Lambda} \epsilon^{\mathrm{abd}} D_{\mathrm{dc}}(A) \\
\left\{\mathcal{L}^{\mathrm{a}}(A), D_{\mathrm{bc}}(A)\right\} & =\frac{1}{2 \Lambda} \epsilon^{\mathrm{acd}} D_{\mathrm{db}}(A)
\end{aligned}
$$

where $\Pi^{\mathrm{i}}$ are the conjugate momenta to $d^{\mathrm{i}}$. (Because of the symmetric nature of the free Hamiltonian, the same brackets are true if we replace $A$ by $B$ everywhere. Furthermore all the mixed brackets between $A$ and $B$ are zero.)

In the case where $C^{\mathrm{k}}$ is $\mathcal{R}^{\mathrm{k}}(A), \mathcal{L}^{\mathrm{k}}(A), \mathcal{R}^{\mathrm{k}}(B)$ or $\mathcal{L}^{\mathrm{k}}(B)$, we get

$$
\begin{aligned}
& \frac{\mathrm{d}}{\mathrm{dt}} \mathcal{R}^{\mathrm{k}}(A)=\frac{\Delta}{2 M d} \epsilon^{\mathrm{iac}} \epsilon^{\mathrm{jbd}} \mathcal{L}^{\mathrm{c}}(A) \mathcal{L}^{\mathrm{d}}(B)\left(\delta^{\mathrm{ij}}-\hat{d}^{\mathrm{i}} \hat{d}^{\mathrm{j}}\right) \epsilon^{\mathrm{kef}} D_{\mathrm{fa}}(A) D_{\mathrm{eb}}(B) \\
& +\cdots \\
& \frac{\mathrm{d}}{\mathrm{dt}} \mathcal{R}^{\mathrm{k}}(B)=\frac{\Delta}{2 M d} \epsilon^{\mathrm{iac}} \epsilon^{\mathrm{jbd}} \mathcal{L}^{\mathrm{c}}(A) \mathcal{L}^{\mathrm{d}}(B)\left(\delta^{\mathrm{ij}}-\hat{d}^{\mathrm{i}} \hat{d}^{\mathrm{j}}\right) \epsilon^{\mathrm{kef}} D_{\mathrm{ae}}\left(A^{\dagger}\right) D_{\mathrm{fb}}(B) \\
& +\cdots \\
& \frac{\mathrm{d}}{\mathrm{dt}} \mathcal{L}^{\mathrm{k}}(A)=-\frac{\Delta}{2 M d} \epsilon^{\mathrm{iac}} \epsilon^{\mathrm{jbd}} \mathcal{L}^{\mathrm{d}}(B)\left(\delta^{\mathrm{ij}}-\hat{d}^{\mathrm{i}} \hat{d}^{\mathrm{j}}\right) \times \\
& {\left[\epsilon^{\mathrm{kcf}} \mathcal{L}^{\mathrm{f}}(A) D_{\mathrm{ab}}\left(A^{\dagger} B\right)+\epsilon^{\mathrm{kaf}} D_{\mathrm{fb}}\left(A^{\dagger} B\right) \mathcal{L}^{\mathrm{c}}(A)\right]+\cdots} \\
& \frac{\mathrm{d}}{\mathrm{dt}} \mathcal{L}^{\mathrm{k}}(B)=-\frac{\Delta}{2 M d} \epsilon^{\mathrm{iac}} \epsilon^{\mathrm{jbd}} \mathcal{L}^{\mathrm{c}}(A)\left(\delta^{\mathrm{ij}}-\hat{d}^{\mathrm{i}} \hat{d}^{\mathrm{j}}\right) \times \\
& {\left[\epsilon^{\mathrm{kdf}} \mathcal{L}^{\mathrm{f}}(B) D_{\mathrm{ab}}\left(A^{\dagger} B\right)+\epsilon^{\mathrm{kbf}} D_{\mathrm{af}}\left(A^{\dagger} B\right) \mathcal{L}^{\mathrm{d}}(B)\right]+\cdots}
\end{aligned}
$$

where $\left\{C^{\mathrm{k}}, H_{\mathrm{I}}\right\}_{0}$ is exhibited and the dots represent the remaining very complicated terms which are non zero and actually are not negligible, being of the same order in $1 / d$ as $\left\{C^{\mathrm{k}}, H_{\mathrm{I}}\right\}_{0}$.

Our approximation is reliable as long as the separation $d$ between the particles is large enough for the conjugate momenta to stay close to their free values. As we have already worked with the undeformed product ansatz approximation, and neglected the potential, which are both valid for large $d$, we feel confident that we have not lost any meaningful information by making this further approximation. If $d$ is kept large we should then find geodesics similar (qualitatively at least) to those given by the exact equations of motion.

The system of equations (376), (383), (384), (385) and (386) is still quite complicated and we will treat it with one final approximation method, namely the 
method of variation of constants. This perturbation scheme consists of replacing the variables in the right hand side of these "conservation equations" by their free trajectories. This gives rise to a "variation" of the previously conserved "constants". The procedure can be iterated indefinitely to give higher order corrections. One should however always maintain consistency with the first (Lagrange) approximation. The range of validity of this further approximation is rather hard to define, but it is clear that only slowly varying trajectories in phase space with large $d$ can be considered. The method of "variation of constants" is only useful with respect to the equation for $\vec{d}$, where it gives the scattering trajectory. The change in the spin or the isospin governed by equations (383), (384), (385) and (386) cannot be treated with this approximation method because of the long range nature of the interaction. The results give an infinite change in these angular momenta, which is not reliable. We will get back to this problem in the last subsection of this article.

\subsubsection{Quantization of the Skyrmion spin/isospin states}

We now use the semi-classical Bohr-Sommerfeld quantization rules applied to the classical motion of the free Skyrmion to construct quantum states corresponding to nucleons. We take such an unusual route because we need quantum states which are described by specific classical trajectories and consquently suitable for the approximation of variation of constants. In contrast, the ordinary quantization method does not fit into our scheme since it provides nucleonic states as quantum wave functions. It is nevertheless interesting to consider it in the light of our previous developments and we will briefly describe it in the following paragraph.

Adkins et al [由 quantized the Skyrmion and constructed spin and isospin 1/2 states. They considered the Lagrangian for a single spinning Skyrmion (21) which can be written as

$$
\begin{aligned}
L & =-M+\Lambda \operatorname{tr}\left[\dot{A}^{\dagger} \dot{A}\right] \\
& =-M+2 \Lambda \sum_{\mathrm{i}=0}^{3} \dot{a}_{\mathrm{i}}^{2}
\end{aligned}
$$

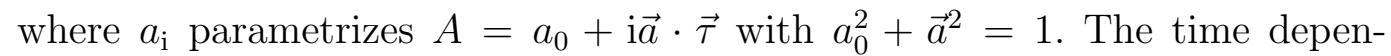
dent SU(2) matrix $A$ defines the rotational characteristics of the Skyrmion. By doing so they make the low energy hypothesis, describing the system approximately using only its zero modes (all radial oscillations, deformations, etc. are neglected). This is exactly like the BPS case: geodesic motion on the minimum energy manifold. One must solve 37 the Schrödinger equation on the manifold of low energy dynamics (in this case, static solutions), finding the wave functions for each state of the system in a highly non-Cartesian (i.e. 
curved) moduli space. The one Skyrmion system is a very simple and elegant illustration of this method.

By considering the truncation of the system (387), the configuration of the system is just represented by a point moving on the 3 -sphere of the $\mathrm{SU}(2)$ group defined by the familiar constraint:

$$
A A^{\dagger}=1=a_{0}^{2}+\vec{a}^{2}
$$

This is a straightforward generalization of the system studied in the first example of subsection 4.2.1 where the system was a particle following great circles on a 2 -sphere. By computing $\vec{\pi}_{\mathrm{a}}$ (the variables canonically conjugate to the $\vec{a}$ )

$$
\pi_{\mathrm{a}}^{\mathrm{i}}=\frac{\partial L}{\partial \dot{a}_{\mathrm{i}}}
$$

substituting in the Lagrangian (387), and performing the usual Legendre transformation, we obtain the familiar expression for the Hamiltonian of a spinning rigid body of mass $M$ and moment of inertia $\Lambda$ :

$$
H=M+\frac{1}{2 \Lambda} \vec{\pi}_{\mathrm{a}}^{2}
$$

The usual canonical quantization procedure gives:

$$
H=M-\frac{1}{2 \Lambda} \sum_{\mathrm{i}=0}^{3} \frac{\partial^{2}}{\partial a_{\mathrm{i}}^{2}}
$$

which is the Laplacian over the 3-sphere of $\mathrm{SU}(2)$ : the Schrödinger equation we have to solve on the low-energy manifold comes naturally. Solutions to this equation are well known: symmetrical, traceless polynomials in $a_{\mathrm{i}}$. Statistical considerations impose the order of the polynomial to be odd for the Skyrmion to be a fermion, and even for a boson. Adkins et al [4] of course chose the former. They found the following states four spin/isospin states:

$$
\begin{aligned}
& |p \uparrow\rangle=\frac{1}{\pi}\left(a_{1}+\mathrm{i} a_{2}\right) \\
& |p \downarrow\rangle=-\frac{\mathrm{i}}{\pi}\left(a_{0}-\mathrm{i} a_{3}\right) \\
& |n \uparrow\rangle=\frac{\mathrm{i}}{\pi}\left(a_{0}+\mathrm{i} a_{3}\right) \\
& |n \downarrow\rangle=-\frac{1}{\pi}\left(a_{1}-\mathrm{i} a_{2}\right)
\end{aligned}
$$


These wave functions are only suitable for a completely quantum mechanical treatment of the Skyrmion-Skyrmion system. As stated earlier we follow the semi-classical route and will obtain these same states via the Bohr-Sommerfeld method[102].

We also start from the classical Lagrangian of equation (387). Using the expression of the matrix $A$ as a function of the usual Euler angles:

$$
A=e^{-\mathrm{i} \alpha \tau_{3} / 2} e^{-\mathrm{i} \beta \tau_{2} / 2} e^{-\mathrm{i} \gamma \tau_{3} / 2}
$$

where $\alpha, \gamma \in[0,2 \pi]$ and $\beta \in[0, \pi]$, or for the $a_{\mu}$

$$
\begin{aligned}
& a_{0}=\cos \left(\frac{\beta}{2}\right) \cos \left(\frac{\alpha+\gamma}{2}\right) \\
& a_{1}=\sin \left(\frac{\beta}{2}\right) \sin \left(\frac{\alpha-\gamma}{2}\right) \\
& a_{2}=-\sin \left(\frac{\beta}{2}\right) \cos \left(\frac{\alpha-\gamma}{2}\right) \\
& a_{3}=-\cos \left(\frac{\beta}{2}\right) \sin \left(\frac{\alpha+\gamma}{2}\right),
\end{aligned}
$$

we find in another form the Lagrangian for a rotating rigid body:

$$
L=-M+\frac{1}{2} \Lambda\left[\dot{\alpha}^{2}+\dot{\beta}^{2}+\dot{\gamma}^{2}+2 \dot{\alpha} \dot{\gamma} \cos \beta\right]
$$

The angles $\alpha, \beta$ and $\gamma$ obey the following equations of motion:

$$
\begin{array}{r}
\ddot{\alpha}+\ddot{\gamma} \cos \beta-\dot{\gamma} \dot{\beta} \sin \beta=0 \\
\ddot{\beta}+\dot{\alpha} \dot{\gamma} \sin \beta=0 \\
\ddot{\gamma}+\ddot{\alpha} \cos \beta-\dot{\alpha} \dot{\beta} \sin \beta=0
\end{array}
$$

and refer, in connection with the rigid body system, to the motion of the body fixed axes relative to the laboratory fixed axes. In our case however, they have a different interpretation. Indeed, contrary to an ordinary rigid body which has only one conserved vector quantity in the laboratory reference frame, (namely the total angular momentum) the quantized Skyrmion will have two such conserved quantities: the spin and the isospin. The Euler angles will then specify those two quantities. In fact we can say that the angle $\beta$ roughly will fix the relative orientation of these two vector quantities, while the time derivatives of $\alpha$ and $\gamma$ will fix their magnitudes. For simplicity without loss of generality we will choose the axis of polarization of spin and isospin parallel to the axis 3 of isospace. Following the convention used by Adkins et al 何, we will chose the following expressions for the spin and isospin generators: 


$$
\begin{aligned}
& I_{3}=-2 \Lambda \mathcal{R}_{0}^{3}(A) \equiv-2 \Lambda \mathcal{R}^{3} \\
& S_{3}=2 \Lambda \mathcal{L}_{0}^{3}(A) \equiv 2 \Lambda \mathcal{L}^{3}
\end{aligned}
$$

Following our choice of quantization axes, we take $\beta$ to be either 0 or $\pi$ and look for solutions to the system of equations (402) which are now given by:

$$
\begin{aligned}
& \ddot{\alpha} \pm \ddot{\gamma}=0 \\
& \ddot{\gamma} \pm \ddot{\alpha}=0
\end{aligned}
$$

The variables $\alpha$ and $\gamma$ actually decouple now and the spin and isospin generators are given by (if $\beta$ is constant)

$$
\begin{aligned}
I_{3} & =\Lambda(\dot{\alpha}+\cos \beta \dot{\gamma}) \\
& =\Lambda(\dot{\gamma} \pm \dot{\alpha}) \\
S_{3} & =-\Lambda(\dot{\alpha} \cos \beta+\dot{\gamma}) \\
& =-\Lambda(\dot{\gamma} \pm \dot{\alpha})
\end{aligned}
$$

depending on whether $\beta=0$ ("+" sign) or $\pi$ ("-" sign). It is then easy to choose $\beta$ so that spin and isospin are parallel or antiparallel, and $\alpha$ and $\gamma$ so they are positive or negative. There only remains to apply the quantization rules of Bohr-Sommerfeld to the system. In the original problem of the hydrogen atom, this method was used to compute the allowed radii of the electron orbits. In the case of the Skyrmion, the radius of the orbits is already defined since the system moves at constant angular velocity along trajectories which are the great circles of $\mathrm{SU}(2)$, therefore having radius 1 . The quantization condition will fix the angular velocity so that the spin and isospin have the right value, namely $1 / 2$ for nucleons, $3 / 2$ for the first nucleon resonance and so on. The Bohr-Sommerfeld quantization condition, derived by path integral methods, is 103

$$
W=\sum_{\mathrm{i}} J_{\mathrm{i}}=(n+\xi) h, \quad n=0, \pm 1, \pm 2, \cdots
$$

where the $J_{\mathrm{i}}$ are the action angle variables and $\xi$ is a correction factor arising from the functional integral over Gaussian fluctuations about the classical trajectory, which we will neglect. The action-angle variables $J_{\mathrm{i}}$ are defined by

$$
J_{\mathrm{i}}=\oint p_{\mathrm{i}} d q_{\mathrm{i}}
$$

where $p_{\mathrm{i}}$ is the momentum conjugate to the coordinate $q_{\mathrm{i}}$, and the integral is taken along a closed path followed by the system during one period in the 
plane $\left(q_{\mathrm{i}}, p_{\mathrm{i}}\right)$ of phase space. In our case $q_{\mathrm{i}}$ represents the angles $\alpha, \beta$ and $\gamma$. Due to the cyclic nature of the angles $\alpha$ and $\gamma, J_{\alpha}$ and $J_{\gamma}$ are readily computed:

$$
\begin{aligned}
& J_{\alpha}=\oint p_{\alpha} \mathrm{d} \alpha=2 \pi \mathrm{p}_{\alpha}=2 \pi \Lambda[\dot{\alpha}+\dot{\gamma} \cos \beta] \\
& J_{\gamma}=\oint p_{\gamma} \mathrm{d}_{\gamma}=2 \pi \mathrm{p}_{\gamma}=2 \pi \Lambda[\dot{\gamma}+\dot{\alpha} \cos \beta] .
\end{aligned}
$$

and by fixing $\beta$ to either 0 or $\pi, J_{\beta}$ is 0 . Then we find, applying the quantization rules,

$$
J_{\alpha}+J_{\gamma}=n h, \quad n=0, \pm 1, \pm 2, \cdots .
$$

The spin and isospin generators can also be expressed as functions of the action angle variables using (407), (408), (411) and (412):

$$
\begin{aligned}
I_{3} & =\frac{J_{\alpha}}{2 \pi} \\
S_{3} & =-\frac{J_{\gamma}}{2 \pi} .
\end{aligned}
$$

By symmetry, the Skyrmion has equal magnitude of spin and isospin, $\left|I_{3}\right|=$ $\left|J_{3}\right|$, so for $n=1$ in (413) we have $\left|J_{\alpha}\right|=\left|J_{\gamma}\right|=1 / 2$.

In the case where $\beta=0$, then using (407) and 408)

$$
\begin{aligned}
& I_{3}=\Lambda(\dot{\alpha}+\dot{\gamma}) \equiv 2 \Lambda \omega= \pm 1 / 2 \\
& S_{3}=-\Lambda(\dot{\alpha}+\dot{\gamma}) \equiv-2 \Lambda \omega=\mp 1 / 2
\end{aligned}
$$

with $(\alpha+\gamma) / 2=\phi(t)=\omega t+\phi_{0}$, a solution of (405) and (406). This type of angular motion produces Skyrmions with spin and isospin antiparallel, and the states $\mid p \downarrow>$ and $\mid n \uparrow>$. The proton state corresponds to isospin $+1 / 2$ along the 3 axis in isospace while the neutron corresponds to isospin $-1 / 2$. Replacing this solution in equation (396) gives

$$
\begin{aligned}
A & =\cos \phi(t)-\mathrm{i} \sin \phi(\mathrm{t}) \tau^{3} \\
& =e^{-\mathrm{i} \phi(\mathrm{t}) \tau^{3} / 2}
\end{aligned}
$$

where $\omega>0$ corresponds to the state $\mid p \downarrow>$ and $\omega<0$ to $\mid n \uparrow>$.

In the case where $\beta=\pi$, 


$$
\begin{gathered}
I_{3}=\Lambda(\dot{\alpha}-\dot{\gamma}) \equiv 2 \Lambda \omega= \pm 1 / 2 \\
S_{3}=-\Lambda(\dot{\gamma}-\dot{\alpha}) \equiv 2 \Lambda \omega= \pm 1 / 2
\end{gathered}
$$

with $(\alpha-\gamma) / 2=\psi(t)=\omega t+\psi_{0}$ and the corresponding matrix

$$
\begin{aligned}
A & =\mathrm{i}\left[\sin \psi(\mathrm{t}) \tau^{1}-\cos \psi(\mathrm{t}) \tau^{2}\right] \\
& =-\mathrm{ie}^{-\mathrm{i} \psi(\mathrm{t}) \tau^{3} / 2} \tau^{2} \mathrm{e}^{\mathrm{i} \psi(\mathrm{t}) \tau^{3} / 2}
\end{aligned}
$$

represents the state $\mid p \uparrow>$ with $\omega>0$ and $\mid n \downarrow>$ with $\omega<0$. We can see the similarity of the forms for the matrices $A$ corresponding to nucleon states and

the wave functions obtained in the directly quantum version of Adkins et al (395). Finally there remains to compute the magnitude of $\omega$ so as to have spin and isospin $1 / 2$. This is done by solving the following equation:

$$
\left|I_{3}\right|=\frac{1}{2}=2 \Lambda|\omega| \Longrightarrow \omega=\frac{1}{4 \Lambda} \sim 50-100 \mathrm{MeV}
$$

using the value of $\Lambda$ from reference[20]. The uncertainty is caused by the values of $f_{\pi}$ and $e$ (via the moment of inertia $\Lambda$ ) which are subject to variations depending on which observables are chosen to be best reproduced by the model.

\subsubsection{Nucleon-nucleon scattering}

We are now ready to compute nucleon-nucleon scattering trajectories. As mentioned earlier, we use the approximation method of "variation of constants" and simply replace into the right hand side of equations (376), (383), (384) (385) and (386) the semi-classically quantized trajectories found in the previous section for spin and isospin, as well as taking $\vec{d}(t)$ corresponding to its free trajectory, a straight line at constant velocity. To calculate the change induced in the previously constant quantities we integrate the equations from $t=-\infty$ to $t=+\infty$ over one free trajectory. This will enable us to obtain scattering angles for the trajectories.

As we mentioned earlier, this computation scheme does not work for the time evolution of the spins and isospins. Equations (383), (384), (385) and (386) all have on the right hand side a factor $1 / d$ which behaves like $1 / t$ for large values of $t$ since $\vec{d}(t)=\vec{v} t+\vec{\gamma}$. Then

$$
\frac{\mathrm{d}}{\mathrm{dt}} \mathcal{L}^{\mathrm{k}} \sim \frac{1}{d} \sim \frac{1}{t}
$$

and similarly for $\mathcal{R}^{\mathrm{k}}$. Then $\mathcal{R}$ and $\mathcal{L}$ change by an infinite amount between $t=-\infty$ and $t=+\infty$ (because the right hand side of the equations integrate 
to a log which varies very slowly). This means that our approximation scheme is too crude or that our treatment is not valid for zero pion mass. Indeed, if $m_{\pi} \neq 0$ then the usual Yukawa cut-off factors $e^{-m_{\pi} r}$ will arise on the right hand side of the equations of motion, and will render the changes in $\mathcal{L}$ and $\mathcal{R}$ finite. There does not seem to be any easy solution to the problem so we will not discuss spin/isospin changes further. We will just say that results are (roughly) compatible with the exchange of charge carrying (pions) and spin carrying (vector mesons) as intermediate particles.

By contrast, equation (376) shows that $\mathrm{d} / \mathrm{d} t \dot{d}^{\mathrm{i}}$ behaves like $1 / d^{2}$, and the approximation method works well. We present below the results for the scattering of nucleons for some particular cases of the initial polarizations, using our semi-classical formalism, namely scattering of particles whose spin and isospin are polarized along the $z$ axis

$$
\begin{array}{r}
A(t), B(t)=\cos \phi(t)-\mathrm{i} \sin \phi(\mathrm{t}) \tau^{3} \text { for the states } \mid \mathrm{p} \downarrow>\text { and } \mid \mathrm{n} \uparrow> \\
\text { or } \mathrm{i}\left[\sin \psi(\mathrm{t}) \tau^{1}-\cos \psi(\mathrm{t}) \tau^{2}\right] \text { for the states } \mid \mathrm{p} \uparrow>\text { and } \mid \mathrm{n} \downarrow>
\end{array}
$$

and the relative motion initially given by

$$
\vec{d}(t)=\vec{v} t+\vec{\gamma}
$$

where $\vec{\gamma}$ is the impact parameter vector if $\vec{v}$ and $\vec{\gamma}$ are chosen orthogonal and the time of closest approach is at $t=0$. These give simple two-dimensional orbits which can be obtained analytically. The tensorial nature of the interaction implies that the forces depend on the angle between the axis of separation and the spin polarization. If we choose the spin polarization along an axis tilted with respect to the normal to the initial scattering plane we get complicated, three dimensional scattering trajectories.

It is important to observe that in our formalism an additional parameter arises which describes the initial state of two incoming, polarized nucleons. This parameter, along with the impact parameter, the initial velocity and the direction of polarization, actually selects the particular scattering trajectory followed by the nucleons. The parameter describes the relative orientation of the Skyrmions at a fixed (initial) time. It plays in fact a role similar to a hidden variable. An incoming pair of physical nucleons, in our formalism, has a fixed value for this parameter, which is only "measured" after the scattering takes place. In a physical experiment consisting of incoming beams of nucleons giving rise to collisions or scattering of pairs of nucleons, the value of this parameter will be uniformly distributed. A similar parameter arises in the case of the scattering of BPS monopoles. This parameter enters the computations via $\delta$ and $\epsilon$ which are defined by 


$$
\begin{aligned}
& \delta=\phi_{0}^{\mathrm{A}}-\phi_{0}^{\mathrm{B}} \\
& \epsilon=\phi_{0}^{\mathrm{A}}+\phi_{0}^{\mathrm{B}}
\end{aligned}
$$

in self evident notation.

There is an immediate separation of the scattering into two cases, depending on whether $D_{\mathrm{ab}}\left(A^{\dagger} B\right)$ is time independent or not. When it depends on time for large values of the ratio $(\omega \gamma / v)$ there is an exponential suppression of the scattering where $v$ is the relative velocity and $\gamma$ the impact parameter. This is quite evident: for slowly translating Skyrmions, the prescribed rotations imposed by selecting semi-classically quantized nucleon states have the effect of averaging the interaction to zero. Interactions which depend on the relative orientation of rapidly spinning bodies are common in the classical or semi-classical treatment of soliton systems. A similar numerical example is presented by Piette et al [104] in the case of "baby-Skyrmions" (Skyrmions in a two dimensional space), where classical trajectories and scattering of spinning solitons is studied. They too exhibit an "oscillatory" interaction which nonetheless generates a net force between the particles and non-trivial scattering.

First case: $D_{\mathrm{ab}}\left(A^{\dagger} B\right)$ time independent We first present the expression for time variations of the previously constant relative momentum $\vec{p}=(M / 2) \dot{\vec{d}}$ for the scattering of protons or of neutrons with various spin polarisations.

$$
\begin{aligned}
& p \uparrow p \uparrow \\
& n \downarrow n \downarrow \quad \frac{\mathrm{d}}{\mathrm{dt}} p^{\mathrm{k}}=-\frac{\Delta \omega^{2}}{d^{2}} \cos (2 \delta) \hat{d}^{\mathrm{k}} \\
& p \downarrow p \downarrow \\
& n \uparrow n \uparrow
\end{aligned}
$$

(ii) $\quad p \uparrow p \downarrow$

$$
\begin{aligned}
& \frac{\mathrm{d}}{\mathrm{dt}} p^{\mathrm{k}}=-\frac{\Delta \omega^{2}}{d^{2}}\left[\hat{d}^{\mathrm{k}}+4 r^{\mathrm{k}} \hat{r} \cdot \hat{d}-6 \hat{d}^{\mathrm{k}}(\hat{r} \cdot \hat{d})^{2}\right] \\
& \hat{r}^{\mathrm{k}}=(-\sin (\delta), \cos (\delta), 0) \\
& \frac{\mathrm{d}}{\mathrm{dt}} p^{\mathrm{k}}=-\frac{\Delta \omega^{2}}{d^{2}}\left[\hat{d}^{\mathrm{k}}+4 r^{\mathrm{k}} \hat{r} \cdot \hat{d}-6 \hat{d}^{\mathrm{k}}(\hat{r} \cdot \hat{d})^{2}\right] \\
& \hat{r}^{\mathrm{k}}=(\sin (\delta),-\cos (\delta), 0)
\end{aligned}
$$

The right hand sides can be interpreted, at this level of our approximation, as coming from a spin-spin channel and a tensor channel interaction. We stress that this is only a correspondence: the true effect of the kinetic term is to supply a non-trivial connection in the geodesic equations on the low energy sub-manifold and not to modify the potential. 
To find the actual change in $\vec{p}$ and hence the scattering angle, we integrate these equations from $t=-\infty$ to $t=+\infty$. For the cases of scattering of protons or neutrons on each other respectively we find that the scattering angle depends on the variable $\delta$ which corresponds to the phase lag between the rotation of $A(t)$ and $B(t)$. Straightforward integration gives

$$
\begin{gathered}
p^{\mathrm{k}}(t)=-\Delta \omega^{2} \cos (2 \delta) \int_{-\infty}^{t} \frac{v^{\mathrm{k}} t+\gamma^{\mathrm{k}}}{\left(v^{2} t^{2}+\gamma^{2}\right)^{3 / 2}} \mathrm{~d} t+\frac{M}{2} v^{\mathrm{k}} \\
=-\Delta \omega^{2} \cos (2 \delta)\left[-\frac{v^{\mathrm{k}}}{v^{2}\left(v^{2} t^{2}+\gamma^{2}\right)^{1 / 2}}+\frac{\gamma^{\mathrm{k}} t}{\gamma^{2}\left(v^{2} t^{2}+\gamma^{2}\right)^{1 / 2}}\right]_{-\infty}^{t} \\
+\frac{M}{2} v^{\mathrm{k}} .
\end{gathered}
$$

This yields

$$
p^{\mathrm{k}}(+\infty)=-2 \Delta \omega^{2} \cos 2 \delta \frac{\gamma^{\mathrm{k}}}{\gamma^{2} v}+\frac{M}{2} v^{\mathrm{k}}
$$

from which we calculate the cosine of the scattering angle

$$
\begin{aligned}
\cos \theta & =\frac{\vec{p}(+\infty) \cdot \vec{p}(-\infty)}{|\vec{p}(+\infty)||\vec{p}(-\infty)|} \\
& =\frac{M \gamma v^{2}}{4 \Delta} \frac{1}{\left(\frac{M^{2} \gamma^{2} v^{4}}{16 \Delta^{2}}+\omega^{4} \cos ^{2} 2 \delta\right)^{1 / 2}}
\end{aligned}
$$

Second case: $D_{\mathrm{ab}}\left(A^{\dagger} B\right)$ time dependent For the cases of collisions between protons and neutrons, the expression for $p^{k}(+\infty)$ is more complicated, because of the time dependence of $D_{\mathrm{ab}}\left(A^{\dagger} B\right)$. The expressions for the scattering of $p$ on $n$ each contain a time dependent $A^{\dagger} B$. When integrated these yield an exponentially suppressed variation in the dimensionless group $(\omega \gamma / v)$

$$
\sim e^{-\left(\frac{\omega \gamma}{v}\right)}
$$

Thus in the limit $v \rightarrow 0$ we get negligible scattering in these cases. These exponential suppression factors appear in the solutions via $G$ functions which can be expressed from Bessel functions by

$$
G_{\mathrm{n}, \mathrm{m}}(x, y)=\frac{\mathrm{d}^{\mathrm{n}}}{\mathrm{d} x^{\mathrm{n}}} x^{\mathrm{m}} K_{\mathrm{m}}(x, y)
$$


where $K_{\mathrm{m}}(x)$ is the Bessel function of the second kind. We get

(i) $\quad \begin{aligned} & p \uparrow n \downarrow \\ & p \downarrow n \uparrow\end{aligned} \quad \frac{\mathrm{d}}{\mathrm{dt}} p^{\mathrm{k}}=\frac{\Delta \omega^{2}}{d^{2}} \cos (4 \omega t+2 \epsilon) \hat{d}^{\mathrm{k}}$

Which gives after integration

$$
\begin{aligned}
& p_{\mathrm{x}}(+\infty)=\frac{2 \Delta \omega^{2}}{M \gamma v} \sin 2 \epsilon G_{1,1}(4 \omega, \gamma / v)+\frac{M v}{2} \\
& p_{\mathrm{y}}(+\infty)=\frac{2 \Delta \omega^{2}}{M v^{2}} \cos 2 \epsilon G_{0,1}(4 \omega, \gamma / v) . \\
& (i i) \quad p \uparrow n \uparrow \quad \frac{\mathrm{d}}{\mathrm{dt}} p^{\mathrm{k}}=\frac{\Delta \omega^{2}}{d^{2}}\left[\hat{d}^{\mathrm{k}}+4 r^{\mathrm{k}} \hat{r} \cdot \hat{d}-6 \hat{d}^{\mathrm{k}}(\hat{r} \cdot \hat{d})^{2}\right] \\
& \hat{r}^{\mathrm{k}}=(-\sin (2 \omega t+\epsilon), \cos (2 \omega t+\epsilon), 0)
\end{aligned}
$$

similarly integrates to

$$
\begin{gathered}
p_{\mathrm{x}}(+\infty)=-\frac{2 \Delta \omega^{2}}{3} \sin 2 \epsilon\left[\frac{8 \gamma}{v^{3}} G_{0,2}(4 \omega, \gamma / v)+\frac{20}{v^{2}} G_{1,2}(4 \omega, \gamma / v)\right. \\
\left.+\frac{16}{\gamma v} G_{2,2}(4 \omega, \gamma / v)+\frac{4}{\gamma^{2}} G_{3,2}(4 \omega, \gamma / v)\right]+\frac{M v}{2} \\
p_{\mathrm{y}}(+\infty)=-\frac{2 \Delta \omega^{2}}{3} \cos 2 \epsilon\left[\frac{4 \gamma}{v^{3}} G_{0,2}(4 \omega, \gamma / v)+\frac{16}{v^{2}} G_{1,2}(4 \omega, \gamma / v)\right. \\
\left.+\frac{20}{\gamma v} G_{2,2}(4 \omega, \gamma / v)+\frac{8}{\gamma^{2}} G_{3,2}(4 \omega, \gamma / v)\right]
\end{gathered}
$$

and

(iii) $\quad p \downarrow n \downarrow \quad \frac{\mathrm{d}}{\mathrm{dt}} p^{\mathrm{k}}=\frac{\Delta \omega^{2}}{d^{2}}\left[\hat{d}^{\mathrm{k}}+4 r^{\mathrm{k}} \hat{r} \cdot \hat{d}-6 \hat{d}^{\mathrm{k}}(\hat{r} \cdot \hat{d})^{2}\right]$
$\hat{r}^{\mathrm{k}}=(-\sin (2 \omega t+\epsilon),-\cos (2 \omega t+\epsilon), 0)$

to

$$
\begin{array}{r}
p_{\mathrm{x}}(+\infty)=-\frac{2 \Delta \omega^{2}}{3} \sin 2 \epsilon\left[\frac{8 \gamma}{v^{3}} G_{0,2}(4 \omega, \gamma / v)-\frac{20}{v^{2}} G_{1,2}(4 \omega, \gamma / v)\right. \\
\left.+\frac{16}{\gamma v} G_{2,2}(4 \omega, \gamma / v)-\frac{4}{\gamma^{2}} G_{3,2}(4 \omega, \gamma / v)\right]+\frac{M v}{2} \\
p_{\mathrm{y}}(+\infty)=-\frac{2 \Delta \omega^{2}}{3} \cos 2 \epsilon\left[-\frac{4 \gamma}{v^{3}} G_{0,2}(4 \omega, \gamma / v)+\frac{16}{v^{2}} G_{1,2}(4 \omega, \gamma / v)\right. \\
\left.\quad-\frac{20}{\gamma v} G_{2,2}(4 \omega, \gamma / v)+\frac{8}{\gamma^{2}} G_{3,2}(4 \omega, \gamma / v)\right]
\end{array}
$$

This is to our knowledge the first analytical calculation of nucleon-nucleon scattering from essentially first principles, without recourse to ad hoc models or potentials. To calculate the classical scattering cross-section we need to compute the scattering for all different polarizations relative to the initial 
scattering plane. This would comprise a different project which would probably be best achieved by numerical methods. Therefore we are unable at this point to make a direct comparison with experiment.

Let us now make a few remarks on our results. In the limit that the initial velocity vanishes, for fixed $\omega$ and $\gamma$, we recover $90^{\circ}$ scattering. This is, however, not surprising as it is a property also shared by the Coulomb and many other interactions treated within our approximation. $90^{\circ}$ scattering is hardly remarkable except at zero impact parameter, where of course, it is impossible to avoid the region of close proximity of the nucleons and it is important that the configurations pass through the minimal, toroidal configuration.

We have made several approximations in our treatment, which deserve some discussion. First we want consider the method of variation of constants. To check its accuracy, we observe that equation (430) is a simple Kepler problem (for this particular equation). We solve it directly to find the exact value of the scattering angle and then compare wiith the result obtained by variation of constants. The exact scattering angle $\Theta$ is:

$$
\cos \Theta=1-\frac{2}{1+\frac{\gamma^{2} M^{2} v^{4}}{4 \Delta^{2} \omega^{4} \cos ^{2} 2 \delta}} .
$$

Defining a parameter $\chi$

$$
\chi=\frac{\cos ^{2} 2 \delta \Delta^{2} \omega^{4}}{\gamma^{2} M^{2} v^{4}},
$$

and choosing $v$ and $\gamma$ so that $\chi$ is much smaller than 1 we get from equations (437) and (446):

$$
\begin{aligned}
& \cos \theta=\frac{1}{\sqrt{1+16 \chi}} \simeq 1-8 \chi+O\left(\chi^{2}\right) \\
& \cos \Theta=\frac{1-4 \chi}{1+4 \chi} \simeq 1-8 \chi+O\left(\chi^{2}\right) .
\end{aligned}
$$

With the previously defined values and definitions we get

$$
\chi \sim 10^{-10} \mathrm{MeV}^{-2} \times \frac{1}{\gamma^{2} v^{4}} \ll 1 .
$$

This imposes very loose restrictions to $v$ and $\gamma$. For these conditions, the approximation seems to work well and respect the other assumptions.

The second main approximation is the expansion in inverse powers of $d$, neglecting all terms beyond the dominant contribution (from the kinetic term). 
We have from the start restricted ourselves to large separations between the Skyrmions. In this regime, the Skyrmions are well described to leading order by the product ansatz and the manifold of collective coordinates is parametrized by the variables of the product ansatz. The induced metric, calculated to leading order behaves as $1 / d$ while the induced potential behaves as $1 / d^{3}$. In principle there can be a region where the contribution of the induced metric dominates and we can neglect the potential. We find that the metric induces an interaction which can be interpreted, within our approximation method, as a spin-spin and a tensor interaction. Unfortunately it seems that the domination by the metric term is not physically realized. The induced kinetic term is multiplied by essentially the frequencies of angular rotation of the Skyrmions:

$$
\begin{aligned}
T_{\mathrm{int}} & =\frac{2 \pi \kappa^{2} f_{\pi}^{2}}{d} \epsilon^{\mathrm{iac}} \epsilon^{\mathrm{jbd}} \mathcal{L}^{\mathrm{a}}(A) \mathcal{L}^{\mathrm{d}}(B)\left(\delta^{\mathrm{ij}}-\hat{d}^{\mathrm{i}} \hat{d}^{\mathrm{j}}\right) D_{\mathrm{ab}}\left(A^{\dagger} B\right)+O\left(1 / d^{2}\right) \\
& \sim \frac{2 \pi \kappa^{2} f_{\pi}^{2}}{d} \omega^{2}
\end{aligned}
$$

while the potential term has two extra powers of the separation in the denominator:

$$
\begin{aligned}
V_{\text {int }} & =4 \pi f_{\pi}^{2} \kappa^{2} \frac{(1-\cos \theta)\left(3(\hat{n} \cdot \hat{d})^{2}-1\right)}{d^{3}} \\
& \sim \frac{4 \pi f_{\pi}^{2} \kappa^{2}}{d^{3}} .
\end{aligned}
$$

So

$$
\begin{aligned}
H_{\mathrm{int}} & =T_{\mathrm{int}}+V_{\mathrm{int}} \\
& \simeq \frac{2 \pi \kappa^{2} f_{\pi}^{2} \omega^{2}}{d}\left(1+\frac{2}{d^{2} \omega^{2}}\right)
\end{aligned}
$$

implies that $T_{\text {int }} \gg V_{\text {int }}$ if

$$
d \gg \frac{\sqrt{2}}{\omega} .
$$

Thus for the kinetic term to dominate, the frequencies should be much larger than the separation. This corresponds to a region of validity for a separation of about $3 \mathrm{fm}$ and greater. However there is much latitude available since the values of $f_{\pi}$ and $e$ which go into determining $\omega$ are fixed only by choosing two experimental inputs. $f_{\pi}$, e can vary as much as $10-30 \%$ thus we do not feel overly concerned with exceeding the regime of validity. Our approximation would of course be better justified for the case of $\Delta-\Delta$ scattering where $\omega \sim 300 \mathrm{MeV}$ corresponding to a separation of $1 \mathrm{fm}$. In any case, we do not 
believe that it is physically reasonable to consider the scattering of nucleons with the metric term alone and we expect a contribution from the potential term which is of the same order of magnitude. We do not expect, however, any great, qualitative modification of the scattering upon inclusion of the potential term, it is of similar strength but actually contributes only in the tensor channel for the case of massless pions.

We have shown how to formulate the nucleon states within the semi-classical approximation. We have treated the scattering and computed the scattering angles in a systematic perturbative approximation. Future work should include consideration of a non-zero pion mass, which leads to a central channel interaction, a better control of the perturbative method, a departure from the product ansatz, and a proper treatment of the region of close proxim-

ity, to test the validity of our formalism in the phenomenology of low energy nucleon-nucleon scattering and of the static quantum states in this sector.

\section{Acknowledgement}

We thank G.E. Brown for inviting us to write this paper and R.B. MacKenzie for his useful comments on the manuscript. This work supported in part by NSERC of Canada and FCAR of Québec.

\section{References}

[1] T.H.R. Skyrme, Proc. Roy. Soc. Lon. 260 (1961) 127-138.

[2] A.P. Balachandran, V.P. Nair, S.G. Rajeev and A. Stern, Phys. Rev. D27 (1983) 1153-1164.

[3] E. Witten, Nucl. Phys. B223 (1983) 422-433.

E. Witten, Nucl. Phys. B223, (1983) 433-444.

[4] G.S. Adkins, C.R. Nappi and E. Witten, Nucl. Phys. B228 (1983) 552-566.

G.S. Adkins and C.R. Nappi, Nucl. Phys. B233 (1984) 109-115.

[5] J. Wess and B. Zumino, Phys. Lett. B37 (1971) 95-97.

[6] S.P. Novikov, Soviet Mathematics Doklady 24 (1981) 222-226.

[7] C. Hadjuk and B. Schwesinger, Phys. Lett. B140 (1984) 172-174.

A. Hayashi and G. Holzwarth, Phys. Lett. B140 (1984) 175-180.

[8] M.S. Sriram, H.S. Mani and R. Ramachandran, Phys. Rev. D30 (1984) 11411143. 
[9] A. Jackson, A.D. Jackson and V. Pasquier, Nucl. Phys. A432 (1985) 567-609.

[10] R. Vinh Mau, M. Lacombe, B. Loiseau, W.N. Cottingham and P. Lisboa, Phys. Lett. B150 (1985) 259-264.

[11] M.P. Mattis and M. Karliner, Phys. rev. D31 (1985) 2833-2848.

M.P. Mattis and M. Mukerjee, Phys. Rev. Lett. 61 (1988) 1344-1347.

[12] I. Klebanov, Nucl. Phys. B262 (1985) 133-143.

A.S. Goldhaber and N.S. Manton, Phys. Lett. 198 (1987) 231-234.

E. Wüst, G.E. Brown and A.D. Jackson, Nucl. Phys. A468 (1987) 450-472.

A.D. Jackson and J.J.M. Verbaarschot, Nucl. Phys. A484 (1988) 419-431.

M. Kugler and S. Shtrikman, Phys. Lett. B208 (1988) 491-494.

M. Kugler and S. Shtrikman, Phys. Rev. D40 (1989) 3421-3429.

L. Castillejo, P.S.J. Jones, A.D. Jackson, J.J.M. Verbaarschot and A.Jackson, Nucl. Phys. A501 (1989) 801-812.

[13] Y. Lu and R.D. Amado, Phys. Rev. C54 (1996) 1566-1573.

[14] Y. Lu, P. Protopapas and R.D. Amado, Phys. Rev. C57 (1998) 1983-1990.

[15] J.J.M. Verbaarschot, T.S. Walhout, J. Wambach and H.W. Wyld, Nucl. Phys. A461 (1987) 603-623.

[16] H.M. Sommermann, R. Seki, S. Larson and S.E. Koonin, Phys. Rev. D45 (1992) 4303-4306.

[17] M.M. Musakhanov and I.V. Musatov, Phys. Lett. B273 (1991) 309-315.

[18] B. Shao, N.R. Walet and R.D. Amado, Phys. Lett. B303 (1993) 1-4.

R.D. Amado, F. Cannata, J.-P. Dedonder, M.P. Locher and B. Shao, Phys. Rev. Lett. 72 (1994) 970-972.

R.D. Amado, F. Cannata, J.-P. Dedonder, M.P. Locher and B. Shao, Phys. Rev. C50 (1994) 640-651.

B. Shao and R.D. Amado, Phys. Rev. C50 (1994) 1787-1795.

R.D. Amado, F. Cannata, J.-P. Dedonder, M.P. Locher and Y. Lu, Phys. Lett. B339 (1994) 201-206.

R.D. Amado, F. Cannata, J.-P. Dedonder, M.P. Locher and Y. Lu, Phys. Rev. C51 (1995) 1587-1590.

Y. Lu and R.D. Amado, Phys. Lett. B357 (1995) 446-450.

[19] I. Zahed and G.E. Brown, Phys. Rep. 142 (1986) 2-102.

[20] T.S. Walhout and J. Wambach, Int. J. Mod. Phys. E1 (1992) 665-738.

[21] M. Rho, Mod. Phys. Lett. A4 (1989) 2571-2587.

[22] V.G. Makhankov, Y.P. Rybakov and V.I. Sanyuk, The Skyrme Model: Fundamentals, Methods, Applications (Springer-Verlag, Berlin, 1993). 
[23] H. Flanders, Differential forms with applications to the physical sciences (Academic Press, New York, 1963).

[24] M. Nakahara, Geometry, topology, and physics (A. Hilger, New York, 1990).

[25] C. Nash and S. Sen, Topology and geometry for physicists (Academic Press, Toronto, 1983).

[26] T. Eguchi, P.B. Gilkey and A.J. Hanson, Phys. Rep. 66 (1980) 213-311.

[27] N.S. Manton and P.J. Ruback, Phys. Lett. 181B (1986) 137-140.

[28] N.S. Manton, Commun. Math. Phys. 111 (1987) 469-478.

[29] M. Loss, Lett. Math. Phys. 14 (1987) 149-156.

[30] M.F. Atiyah and N.S. Manton, Phys. Lett. B222 (1989) 438-442.

[31] M.F. Atiyah and N.S. Manton, Commun. Math. Phys. 152 (1993) 391-422.

[32] R.A. Leese and N.S. Manton, Nucl. Phys. A572 (1994) 575-599.

[33] N.S. Manton and P.M. Sutcliffe, Phys. Lett. B342 (1995) 196-200.

[34] C.J. Houghton, N.S. Manton and P.M. Sutcliffe, Nucl. Phys. B (in press).

[35] K. Isler, J. LeTourneux, and M.B. Paranjape, Phys. Rev. D43 (1991) 13661374 .

[36] N.S. Manton, Phys. Rev. D28 (1983) 2019-2026.

[37] N.S. Manton, Phys. Rev. Lett. 60 (1988) 1916-1919.

[38] G.W. Gibbons and N.S. Manton, Nucl. Phys. B274 (1986) 183-224.

[39] T. Gisiger and M.B. Paranjape, Phys. Rev. D50 (1994) 1010-1015.

[40] T. Gisiger and M.B. Paranjape, Phys. Rev. D51 (1995) 3034-3041.

[41] B.J. Schroers, Z. Phys. C61 (1994) 479-494.

[42] R.W. Ogden, Non-linear elastic deformations (Halsted Press, New York, 1984).

[43] L.D. Landau and E.M. Lifchitz, Théorie de l'élasticité (Editions Mir, Moscou, 1967).

[44] M. Reed and B. Simon, Methods of Modern Mathematical Physics (Academic Press, New York, 1980).

[45] A.D. Jackson, A. Wirzba and L. Castillejo, Phys. Lett. B198 (1987) 315-318.

[46] A.D. Jackson, N.S. Manton and A. Wirzba, Nucl. Phys. A495 (1989) 499-522.

[47] S. Coleman, Aspects of symmetry (Cambridge University Press, New York, 1985).

[48] R. Jackiw, C. Nohl and C. Rebbi, Phys. Rev. D15 (1977) 1642-1646. 
[49] G. 't Hooft, Phys. Rev. Lett. 37 (1976) 8-11.

G. 't Hooft, Phys. Rev. D14 (1976) 3432-3450.

[50] R. Hartshorne, Commun. Math. Phys. 59 (1978) 1-15.

[51] A. Wirzba and H. Bang, Nucl. Phys. A515 (1990) 571-598,

[52] J.J.M. Verbaarschot, Phys. Lett. B195 (1987) 235-239.

[53] N.S. Manton, Phys. Lett. B192 (1987) 177-179.

[54] V.B. Kopeliovich and B.E. Stern, JETP Lett. 45 (1987) 203-207.

[55] A. Hosaka, M. Oka and R.D. Amado, Phys. Lett. B251 (1990) 1-5.

A. Hosaka, M. Oka and R.D. Amado, Nucl. Phys. A530 (1991) 507-531.

[56] J.J.M. Verbaarschot, T.S. Walhout, J. Wambach and H.M. Wyld, Nucl. Phys. A468 (1987) 520-538.

[57] T.S. Walhout, Nucl. Phys. A531 (1991) 596-612.

[58] M.F. Atiyah and N.J. Hitchin, Phys. Lett. A107 (1985) 21-25.

M.F. Atiyah and N.J. Hitchin, Phil. Trans. R. Soc. A315 (1985) 459-469.

[59] R.A. Leese, N.S. Manton and B.J. Schroers, Nucl. Phys. B442 (1995) 228-267.

[60] E. Braaten, S. Townsend, L. Carson, Phys. Lett. B235 (1990) 147-152.

[61] R.A. Battye and P.M. Sutcliffe, Phys. Rev. Lett. 79 (1997) 363-366.

[62] C. Barnes, W.K. Baskerville and N. Turok, Phys. Lett. B411 (1997) 180-186.

[63] C. Barnes, W.K. Baskerville and N. Turok, Phys. Rev. Lett. 79 (1997) 367-370.

[64] W.Y. Crutchfield and J.B. Bell, J. Comp. Phys. 110 (1994) 234-241.

[65] P.M. Sutcliffe, Int. J. Mod. Phys. A12 (1997) 4663-4703.

[66] E.B. Bogomolny, Sov. J. Nucl. Phys. 24 (1976) 449-454.

E.B. Bogomolny, Yad. Fiz. 24 (1976) 861-870.

[67] M.K. Prasad and C.M. Sommerfield, Phys. Rev. Lett. 35 (1975) 760-762

[68] G. 't Hooft, Nucl. Phys. 79 (1974) 276-284.

[69] A.M. Polyakov, JETP Lett. 20 (1974) 194-195.

[70] M.F. Atiyah, N.J. Hitchin, V.G. Drinfeld and Y.I Manin, Phys. Lett. A65 (1978) $185-187$.

[71] N.J. Hitchin, Comm. Math. Phys. 83 (1982) 579-602.

[72] N.S. Manton, Nucl. Phys. B126 (1977) 525-541.

[73] R.S. Ward, Comm. Math. Phys. 79 (1981) 317-325, 
[74] W. Nahm, Phys. Lett. B93 (1980) 42-46.

[75] P. Goddard and D.I. Olive, Rept. Prog. Phys. 41 (1978) 1357-1437.

[76] S. Coleman, The magnetic monopole fifty years later, from the proceedings The unity of the fondamental interactions, Subnuclear Series Vol. 19 (Plenum Press, 1983).

[77] S.K. Donaldson, Commun. Math. Phys. 96 (1984) 387-407.

[78] S. Jarvis, A rational map for Euclidian monopoles via radial scattering (Oxford preprint, 1996).

[79] M. Morse, Global Variational Analysis (Princeton University Press, 1976).

[80] J. Milnor, Morse Theory (Princeton University Press, 1963).

[81] C.H. Taubes, Comm. Math. Phys. 86 (1982) 299-320.

[82] P. Van Baal, Acta. Pol. Phys. B21 (1990) 73-110.

[83] L.A. Ljusternik, The Topology of the Calculus of Variations in the Large, translated by J.M. Danskin (American Mathematical Society, Providence, 1966).

[84] F.R. Klinkhamer and N.S. Manton, Phys. Rev. D30 (1984) 2212-2220.

[85] C. H. Taubes, Progress in Gauge Field Theory, NATO ASI Series, Series B: Physics Vol. 115, Edited by 't Hooft et al (New York: Plenum Press, 1983).

[86] J. Bagger, W. Goldstein and M. Soldate, Phys. Rev. D31 (1985) 2600-2604.

[87] N. Steenrod, The topology of fibre bundles (Princeton University Press: 1951).

[88] A.D. Jackson and M. Rho, Phys. Rev. Lett. 51 (1983) 751-754.

[89] A. Klein, N.R. Walet and G. Do Dang, Ann. Phys. 208 (1991) 90-148.

[90] H. Goldstein, Classical Mechanics, Second Edition (Addison-Wesley Publishing Company, 1980).

[91] T. Waindzoch and J. Wambach, Nucl. Phys. A602 (1996) 347-374.

[92] R.A. Battye and P.M. Sutcliffe, Phys. Lett. B391 (1997) 150-156.

[93] R.B. MacKenzie, Phys. Lett. B352 (1995) 96-98.

[94] J.J.M. Verbaarschot, Proceedings of the Workshop on Skyrmions and Anomalies, Edited by M. Jezabek and M. Praszlowicz (World Scientific, 1987).

[95] P.W. Irwin and N.S. Manton Phys. Lett. B385 (1996) 187-192.

[96] E. M. Nyman and D.O. Riska, Phys. Lett. B203 (1988) 13-17.

[97] T.S. Walhout and J. Wambach, Phys. Rev. Lett. 67 (1991) 314-317.

[98] S.S.M. Wong, Nucl. Phys. A623 (1997) 431-455. 
[99] N.R. Walet and R.D. Amado, Phys. Rev. Lett. 68 (1992) 3849-3852.

N.R. Walet and R.D. Amado, Phys. Rev. C47 (1993) 498-511.

[100] E. Braaten and L. Carson, Phys. Rev. D38 (1988) 3525-3539.

[101] N.S. Manton Phys. Lett. 198B (1987) 226-230

[102] R. Dashen, B. Hasslacher and A. Neveu, Phys. Rev. D10 (1974) 4114-4154.

R. Dashen, Gauge Theories and Modern Field Theory (MIT Press, 1976).

[103] R. Rajaraman, Solitons and Instantons: An Introduction to Solitons and Instantons in Quantum Field Theory, First edition (North-Holland Personal Library 1982).

[104] B.M.A.G. Piette, B.J. Schroers and W.J. Zakrzewski, Nucl. Phys. B439 (1995) 205-235. 